\title{
Ligand-Enabled, Copper-Catalyzed Electrophilic Amination for the Asymmetric Synthesis of $\beta$-Amino Acids
}

\author{
Raj K. Tak, Hidetoshi Noda,* and Masakatsu Shibasaki* \\ Institute of Microbial Chemistry (BIKAKEN), Tokyo, Japan \\ Email: hnoda@bikaken.or.jp; mshibasa@bikaken.or.jp
}

Table of Contents

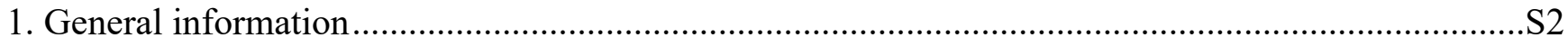

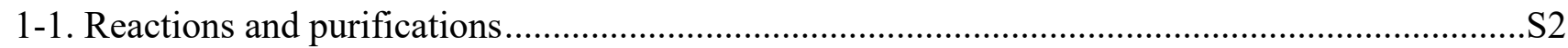

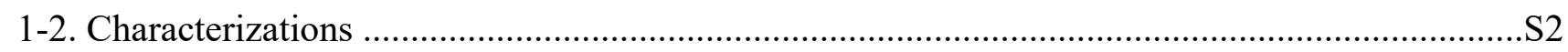

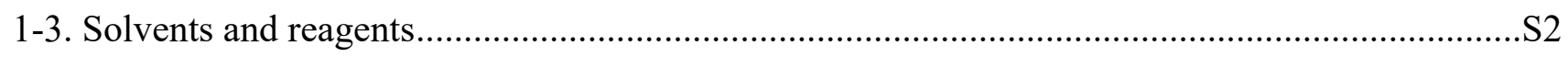

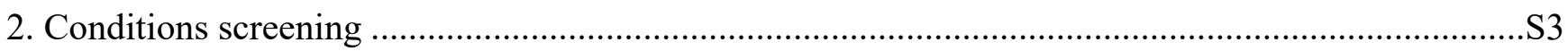

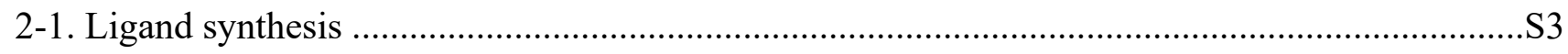

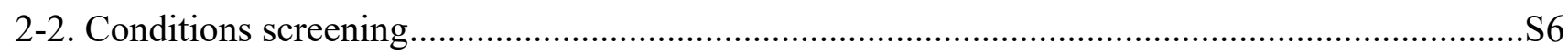

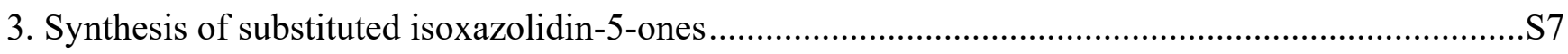

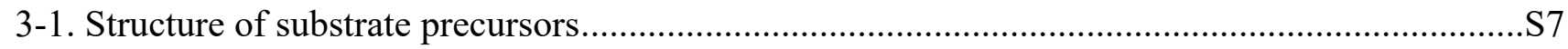

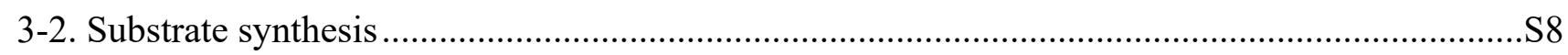

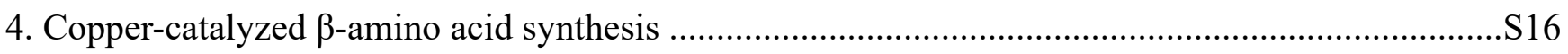

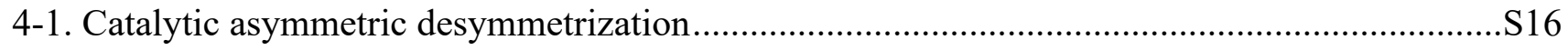

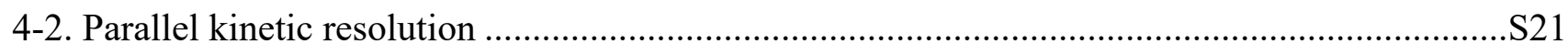

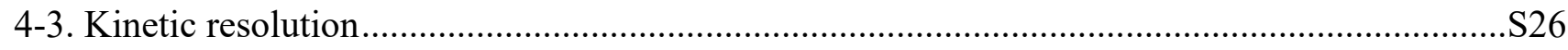

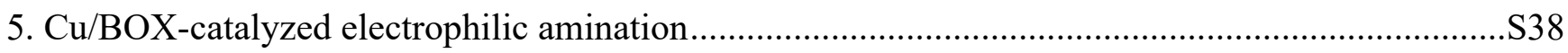

5-1. Limitations of $\mathrm{Cu} / \mathrm{BOX}$-catalyzed asymmetric desymmetrization ........................................S38

5-2. Unexpected product formation under the $\mathrm{Cu} / \mathrm{BOX}$ catalytic conditions ................................S38

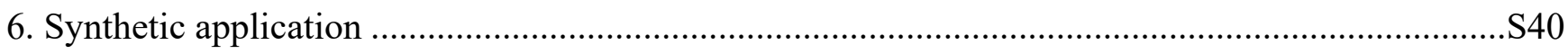

6-1. Large scale catalytic asymmetric desymmetrization .....................................................S40

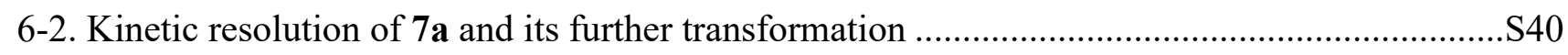

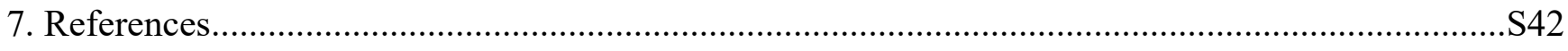

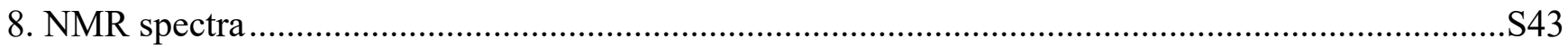




\section{General information}

\section{1-1. Reactions and purifications}

Unless otherwise noted, all reactions were carried out under an argon atmosphere and were stirred with Tefloncoated magnetically stirred bars. All work-up and purification procedures were carried out with reagent-grade solvents under ambient atmosphere. Thin layer chromatography (TLC) was performed on Merck TLC plates $(0.25$ $\mathrm{mm}$ ) pre-coated with silica gel $60 \mathrm{~F} 254$ and visualized by UV quenching and staining with ninhydrin or $\mathrm{KMnO}_{4}$. Flash column chromatography was performed on a Biotage Isolera Spektra One.

\section{1-2. Characterizations}

Infrared (IR) spectra were recorded on a HORIBA FT210 Fourier transform infrared spectrophotometer. NMR spectra were recorded on a Bruker AVANCE III HD400 or a Bruker AVANCE III 600 NMR spectrometer at 298K. Chemical shifts $(\delta)$ are given in ppm relative to residual solvent peaks. ${ }^{1}$ Data for ${ }^{1} \mathrm{H}$ NMR are reported as follows: chemical shift (multiplicity, coupling constants where applicable, number of hydrogens). Abbreviations are as follows: s (singlet), d (doublet), $\mathrm{t}$ (triplet), dd (doublet of doublet), dt (doublet of triplet), ddd (doublet of doublet of doublet), q (quartet), $\mathrm{m}$ (multiplet), br (broad). Optical rotation was measured using a $1.0 \mathrm{~mL}$ cell with a $1.0 \mathrm{dm}$ path length on a JASCO polarimeter P-1030. Melting points were measured on a Yanagimoto Seisakusho Micro Melting Point Apparatus. High-resolution mass spectra (ESI TOF (+)) were measured on a Thermo Fisher Scientific LTQ Orbitrap XL. Normal phase HPLC analysis was conducted on a JASCO HPLC system equipped with Daicel chiral-stationary-phase column $(\phi 0.46 \mathrm{~cm}$ x $25 \mathrm{~cm})$.

\section{1-3. Solvents and reagents}

Unless otherwise noted, materials were purchased from commercial suppliers and were used without further purification. THF and $\mathrm{CH}_{2} \mathrm{Cl}_{2}$ were purified by passing through a solvent purification system (Glass Contour). $\mathrm{CuBr}$ and $\mathrm{CuOTf} \bullet 0.5 \mathrm{C}_{6} \mathrm{H}_{6}$ were purchased from Sigma-Aldrich, and used as received. HFIP was purchased from TCI Co., Ltd., and dried over activated pellet MS4A prior to use. 


\section{Conditions screening}

\section{2-1. Ligand synthesis}

All ligands were synthesized by following the reported procedure. ${ }^{2}$

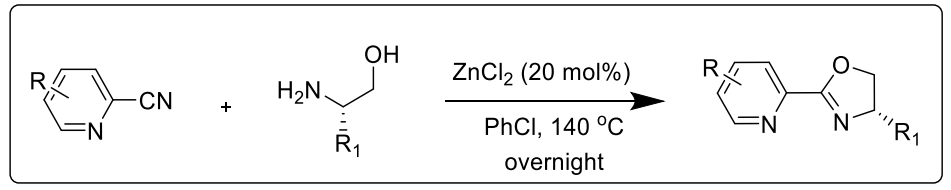

General procedure A: To a $20 \mathrm{~mL}$ round bottom flask was added $\mathrm{ZnCl}_{2}(20 \mathrm{~mol} \%)$, and it was heated with a hot gun under high vacuum for $5 \mathrm{~min}$ and then cooled to room temperature, followed by the charge of argon. To this were added chlorobenzene $(0.5 \mathrm{M})$, picolinonitrile derivative (1.0 equiv) and chiral aminoalcohol (1.5 equiv). The resulting solution was stirred overnight at $140{ }^{\circ} \mathrm{C}$ (oil bath), and cooled to room temperature. After removing all volatiles under reduced pressure, the resulting solid was dissolved in the $\mathrm{CHCl}_{3}$ and the organic layer was washed with water. The aqueous layer was extracted with $\mathrm{CHCl}_{3}(3 \mathrm{x})$ and the combined organic layers were washed with brine, dried over $\mathrm{Na}_{2} \mathrm{SO}_{4}$, filtered and concentrated in vacuo to afford the crude material, which was purified by silica gel column chromatography, eluting with hexane/ethyl acetate.

\section{(S)-4-(tert-Butyl)-5,5-diphenyl-2-(pyridin-2-yl)-4,5-dihydrooxazole (SL1)}

I $\mathrm{C}_{\mathrm{Ph}}^{\mathrm{ph}}$ Prepared by the general procedure A from picolinonitrile (125 mg, $\left.1.2 \mathrm{mmol}\right)$ and $(S)$-2-amino3,3-dimethyl-1,1-diphenylbutan-1-ol (323 mg, $1.2 \mathrm{mmol}$ ), and isolated by silica gel column chromatgraphy (hexane/ethyl acetate) as a colorless liquid (200 mg, 46\% yield). ${ }^{1} \mathbf{H}$ NMR (400 $\left.\mathrm{MHz}, \mathrm{CDCl}_{3}\right): \delta 8.80(\mathrm{ddd}, J=4.8,1.6,0.8 \mathrm{~Hz}, 1 \mathrm{H}), 8.16(\mathrm{dt}, J=7.9,1.0 \mathrm{~Hz}, 1 \mathrm{H}), 7.79(\mathrm{td}, J=7.8,1.7 \mathrm{~Hz}, 1 \mathrm{H})$, $7.68-7.65(\mathrm{~m}, 2 \mathrm{H}), 7.44-7.32(\mathrm{~m}, 5 \mathrm{H}), 7.28-7.26(\mathrm{~m}, 1 \mathrm{H}), 7.25-7.22(\mathrm{~m}, 3 \mathrm{H}), 4.85(\mathrm{~s}, 1 \mathrm{H}), 0.89(\mathrm{~s}, 9 \mathrm{H}) ;{ }^{13} \mathbf{C}$ NMR (101 MHz, $\left.\mathrm{CDCl}_{3}\right): \delta 160.4,150.1,147.2,146.2,140.1,136.5,128.8,128.1,127.7,127.4,127.2,126.6$, 125.4, 124.0, 93.7, 82.9, 77.2, 35.6, 28.0; IR (thin film): 3058, 2953, 2869, 1654, 1568, 1470, 1446, 1353, 1244, 1119, 969, 754, $700 \mathrm{~cm}^{-1}$; HRMS (ESI) $m / z$ calc'd for $\mathrm{C}_{24} \mathrm{H}_{25} \mathrm{ON}_{2}[\mathrm{M}+\mathrm{H}]^{+}: 357.1961$, found: 357.1958; $[\alpha]_{\mathrm{D}}{ }^{27}-$ $353.83\left(c 1.0, \mathrm{CHCl}_{3}\right)$.

\section{(S)-4-(tert-Butyl)-2-(3-chloropyridin-2-yl)-4,5-dihydrooxazole (L1)}

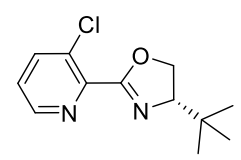

Prepared by the general procedure A from 3-chloropicolinonitrile (169 mg, $1.22 \mathrm{mmol})$ and $(S)$ 2-amino-3,3-dimethylbutan-1-ol (143 $\mathrm{mg}, 1.22 \mathrm{mmol})$, and isolated by silica gel column chromatgraphy (hexane/ethyl acetate) as a white solid (242 mg, 83\% yield). m.p. $37-39{ }^{\circ} \mathrm{C} ;{ }^{1} \mathbf{H}$ NMR (400 MHz, $\left.\mathrm{CDCl}_{3}\right): \delta 8.57(\mathrm{dd}, J=4.6,1.4 \mathrm{~Hz}, 1 \mathrm{H}), 7.79(\mathrm{dd}, J=8.2,1.4 \mathrm{~Hz}, 1 \mathrm{H}), 7.31(\mathrm{dd}, J=8.2,4.6 \mathrm{~Hz}$, $1 \mathrm{H}), 4.43(\mathrm{dd}, J=10.2,8.6 \mathrm{~Hz}, 1 \mathrm{H}), 4.29$ (t, $J=8.4 \mathrm{~Hz}, 1 \mathrm{H}), 4.19(\mathrm{dd}, J=10.2,8.3 \mathrm{~Hz}, 1 \mathrm{H}), 1.00(\mathrm{~s}, 9 \mathrm{H}) ;{ }^{13} \mathrm{C}$ NMR (101 MHz, $\left.\mathrm{CDCl}_{3}\right): \delta 160.3,147.4,145.7,138.5,131.9,125.6,77.2,69.0,33.9,25.6$; IR (thin film): 3053, 
2956, 2869, 1674, 1572, 1442, 1352, 1208, 1114, 1038, 958, 802, $757 \mathrm{~cm}^{-1}$; HRMS (ESI) $\mathrm{m} / z$ calc'd for $\mathrm{C}_{12} \mathrm{H}_{16} \mathrm{ON}_{2} \mathrm{Cl}[\mathrm{M}+\mathrm{H}]^{+}: 239.0946$, found: $239.0944 ;[\alpha]_{\mathrm{D}}{ }^{27}-81.70\left(\right.$ c $\left.1.0, \mathrm{CHCl}_{3}\right)$.

\section{(S)-2-(3-Bromopyridin-2-yl)-4-(tert-butyl)-4,5-dihydrooxazole (SL2)}

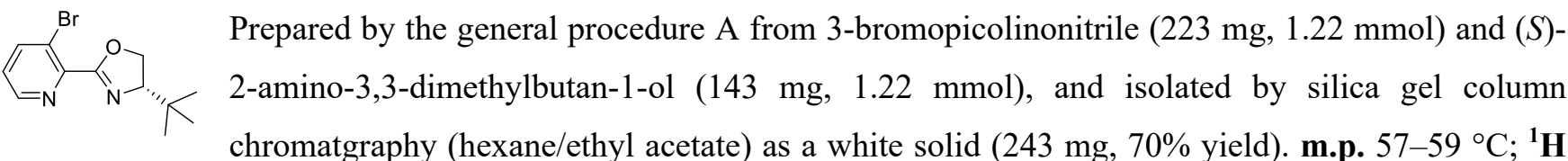
NMR $\left(400 \mathrm{MHz}, \mathrm{CDCl}_{3}\right): \delta 8.61(\mathrm{dd}, J=4.6,1.4 \mathrm{~Hz}, 1 \mathrm{H}), 7.99(\mathrm{dd}, J=8.2,1.4 \mathrm{~Hz}, 1 \mathrm{H}), 7.24(\mathrm{dd}, J=8.2,4.6 \mathrm{~Hz}$, $1 \mathrm{H}), 4.45(\mathrm{dd}, J=10.2,8.5 \mathrm{~Hz}, 1 \mathrm{H}), 4.29$ (t, $J=8.5 \mathrm{~Hz}, 1 \mathrm{H}), 4.19(\mathrm{dd}, J=10.2,8.5 \mathrm{~Hz}, 1 \mathrm{H}), 1.02(\mathrm{~s}, 9 \mathrm{H}) ;{ }^{13} \mathbf{C}$ NMR (101 MHz, $\mathrm{CDCl}_{3}$ ): $\delta 161.1,147.9,147.3,141.7,125.7,120.4,77.2,69.2,34.0,26.1$; IR (thin film): 3050, 2951, 2867, 1671, 1567, 1438, 1334, 1260, 1203, 1108, 1016, 953, 908, 794, $737 \mathrm{~cm}^{-1}$; HRMS (ESI) $\mathrm{m} / z$ calc'd for $\mathrm{C}_{12} \mathrm{H}_{16} \mathrm{ON} 2 \mathrm{Br}[\mathrm{M}+\mathrm{H}]^{+}: 283.0441$, found: 283.0442; $[\alpha]_{\mathrm{D}}^{27}-73.11$ (c 1.0, $\left.\mathrm{CHCl}_{3}\right)$.

\section{(S)-4-(tert-Butyl)-2-(3-phenylpyridin-2-yl)-4,5-dihydrooxazole (SL3)}

Prepared by the general procedure A from 3-phenylpicolinonitrile (219 mg, $1.22 \mathrm{mmol})$ and $(S)$-2-

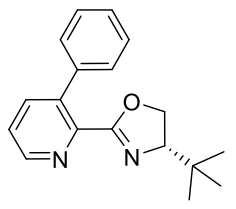
amino-3,3-dimethylbutan-1-ol (143 mg, $1.22 \mathrm{mmol})$, and isolated by silica gel column chromatgraphy (hexane/ethyl acetate) as a colorless oil (245 mg, 72\% yield). ${ }^{1} \mathbf{H}$ NMR (400 MHz, $\left.\mathrm{CDCl}_{3}\right): \delta 8.68(\mathrm{dd}, J=4.7,1.3 \mathrm{~Hz}, 1 \mathrm{H}), 7.72(\mathrm{~d}, J=7.5 \mathrm{~Hz}, 1 \mathrm{H}), 7.42-7.35(\mathrm{~m}, 6 \mathrm{H}), 4.28-4.20$ (m, 1H), $4.02-3.94(\mathrm{~m}, 2 \mathrm{H}), 0.84(\mathrm{~s}, 9 \mathrm{H}) ;{ }^{13} \mathbf{C}$ NMR (101 MHz, $\left.\mathrm{CDCl}_{3}\right): \delta 162.8,148.4,146.3,138.7,138.3$, 129.1, 128.7, 128.2, 127.9, 125.3, 124.6, 77.2, 69.1, 33.7, 26.0; IR (thin film): 3055, 2954, 2868, 1671, 1560, 1446, 1363, 1263, 1196, 1054, 960, 759, $700 \mathrm{~cm}^{-1}$; HRMS (ESI) $\mathrm{m} / z$ calc'd for $\mathrm{C}_{18} \mathrm{H}_{21} \mathrm{ON}_{2}[\mathrm{M}+\mathrm{H}]^{+}: 281.1648$, found: $281.1654 ;[\alpha]_{\mathrm{D}}{ }^{27}-43.41\left(c 1.0, \mathrm{CHCl}_{3}\right)$.

\section{(S)-2-(3-(Benzyloxy)pyridin-2-yl)-4-(tert-butyl)-4,5-dihydrooxazole (SL4)}

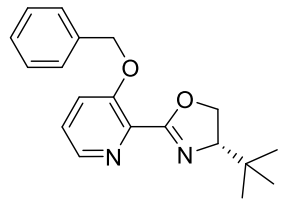

Prepared by the general procedure A from 3-(benzyloxy)picolinonitrile (220 mg, $1.05 \mathrm{mmol}$ ) and (S)-2-amino-3,3-dimethylbutan-1-ol (122 $\mathrm{mg}, 1.05 \mathrm{mmol})$, and isolated by silica gel column chromatgraphy (hexane/ethyl acetate) as a colorless liquid (188 mg, 58\% yield). ${ }^{1} \mathbf{H}$ NMR $\left(400 \mathrm{MHz}, \mathrm{CDCl}_{3}\right): \delta 8.30(\mathrm{dd}, J=4.3,1.4 \mathrm{~Hz}, 1 \mathrm{H}), 7.51(\mathrm{br}, 2 \mathrm{H}), 7.38-7.29(\mathrm{~m}, 5 \mathrm{H})$, $5.19(\mathrm{q}, J=11.8 \mathrm{~Hz}, 2 \mathrm{H}), 4.39(\mathrm{dd}, J=17.8,9.6 \mathrm{~Hz}, 1 \mathrm{H}), 4.24-4.15(\mathrm{~m}, 2 \mathrm{H}), 0.96(\mathrm{~s}, 9 \mathrm{H}) ;{ }^{13} \mathbf{C} \mathbf{N M R}(101 \mathrm{MHz}$, $\left.\mathrm{CDCl}_{3}\right): \delta 160.4,154.6,141.5,137.8,135.9,128.5,128.1,127.3,125.8,121.0,77.2,70.7,68.3,33.9,25.9$; IR (thin film): 3377, 3064, 2959, 2869, 1658, 1577, 1541, 1446, 1365, 1286, 1118, 1054, 985, 802, 741, $699 \mathrm{~cm}^{-1}$; HRMS (ESI) $m / z$ calc'd for $\mathrm{C}_{19} \mathrm{H}_{23} \mathrm{O}_{2} \mathrm{~N}_{2}[\mathrm{M}+\mathrm{H}]^{+}: 311.1754$, found: $311.1759 ;[\alpha]_{\mathrm{D}}{ }^{27}-68.74\left(c 1.0, \mathrm{CHCl}_{3}\right)$. 


\section{(S)-4-(tert-Butyl)-2-(3-(trifluoromethyl)pyridin-2-yl)-4,5-dihydrooxazole (SL5)}

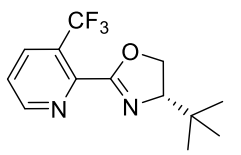

Prepared by the general procedure A from 3-(trifluoromethyl)picolinonitrile (210 mg, $1.22 \mathrm{mmol})$ and $(S)$-2-amino-3,3-dimethylbutan-1-ol (143 mg, $1.22 \mathrm{mmol})$, and isolated by silica gel column chromatgraphy (hexane/ethyl acetate) as a colorless liquid (210 mg, 63\% yield). ${ }^{1} \mathbf{H}$ NMR (400 $\left.\mathrm{MHz}, \mathrm{CDCl}_{3}\right): \delta 8.85(\mathrm{~d}, J=4.3 \mathrm{~Hz}, 1 \mathrm{H}), 8.07(\mathrm{~d}, J=8.1 \mathrm{~Hz}, 1 \mathrm{H}), 7.53-7.50(\mathrm{~m}, 1 \mathrm{H}), 4.49(\mathrm{dd}, J=10.2,8.5 \mathrm{~Hz}$, $1 \mathrm{H}), 4.29(\mathrm{t}, J=9.0 \mathrm{~Hz}, 1 \mathrm{H}), 4.18(\mathrm{t}, J=9.8 \mathrm{~Hz}, 1 \mathrm{H}), 1.00(\mathrm{~s}, 9 \mathrm{H}) ;{ }^{13} \mathbf{C} \mathbf{N M R}\left(101 \mathrm{MHz}, \mathrm{CDCl}_{3}\right): \delta 160.9,152.2$, 146.6, 134.9 (q, $J=4.9 \mathrm{~Hz}), 126.2(\mathrm{q}, J=33.7 \mathrm{~Hz}), 122.8(\mathrm{q}, J=273.4 \mathrm{~Hz}), 77.2,69.9,33.9,26.0 ;{ }^{19} \mathbf{F}$ NMR $(376$ MHz, CDC13): $\delta-60.07$; IR (thin film): 3067, 2958, 2871, 1677, 1575, 1452, 1320, 1145, 1031, 958, 817, $709 \mathrm{~cm}^{-}$

1; HRMS (ESI) $m / z$ calc'd for $\mathrm{C}_{13} \mathrm{H}_{16} \mathrm{ON}_{2} \mathrm{~F}_{3}[\mathrm{M}+\mathrm{H}]^{+}: 273.1209$, found: 273.1210 ; $[\alpha]_{\mathrm{D}}{ }^{27}-34.30\left(c 1.0, \mathrm{CHCl}_{3}\right)$.

\section{(S)-4-(tert-Butyl)-2-(5-methylpyridin-2-yl)-4,5-dihydrooxazole (SL6)}

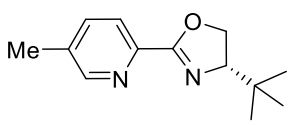

Prepared by the general procedure A from 5-methylpicolinonitrile $(144 \mathrm{mg}, 1.22 \mathrm{mmol})$ and

(S)-2-amino-3,3-dimethylbutan-1-ol (143 mg, $1.2 \mathrm{mmol}$ ), and isolated by silica gel column chromatgraphy (hexane/ethyl acetate) as a white solid (200 mg, 75\% yield). m.p. 87-89 ${ }^{\circ} \mathrm{C} ;{ }^{1} \mathbf{H}$ NMR $(400 \mathrm{MHz}$, $\left.\mathrm{CDCl}_{3}\right): \delta 8.51(\mathrm{dd}, J=1.4,0.6 \mathrm{~Hz}, 1 \mathrm{H}), 7.96(\mathrm{~d}, J=8.0 \mathrm{~Hz}, 1 \mathrm{H}), 7.55(\mathrm{ddd}, J=8.0,2.1,0.6 \mathrm{~Hz}, 1 \mathrm{H}), 4.42(\mathrm{dd}, J=$ 10.2, 8.7 Hz, 1H), $4.28(\mathrm{t}, J=8.5 \mathrm{~Hz}, 1 \mathrm{H}), 4.10(\mathrm{dd}, J=10.2,8.3 \mathrm{~Hz}, 1 \mathrm{H}), 2.37(\mathrm{~s}, 3 \mathrm{H}), 0.96(\mathrm{~s}, 9 \mathrm{H}) ;{ }^{13} \mathbf{C}$ NMR $\left(101 \mathrm{MHz}, \mathrm{CDCl}_{3}\right): \delta 162.6,150.2,144.4,136.9,135.5,123.5,76.5,69.2,34.0,26.0,18.6$; IR (thin film): 2956, 2869, 1645, 1569, 1484, 1356, 1248, 1097, 1029, 966, 909, $845 \mathrm{~cm}^{-1}$; HRMS (ESI) $\mathrm{m} / z$ calc'd for $\mathrm{C}_{13} \mathrm{H}_{19} \mathrm{ON}_{2}[\mathrm{M}$ $+\mathrm{H}]^{+}: 219.1492$, found: $219.1496 ;[\alpha]_{\mathrm{D}}^{27}-80.85\left(c 1.0, \mathrm{CHCl}_{3}\right)$. 


\section{2-2. Conditions screening}

\section{Ligand evaluation}<smiles>O=C1ONCC1(Cc1ccccc1)Cc1ccccc1</smiles>

1a $(0.1 \mathrm{mmol})$

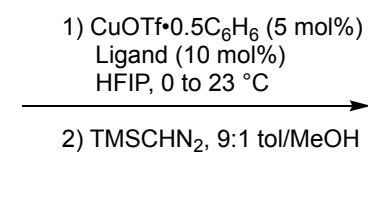

2) $\mathrm{TMSCHN}_{2}, 9: 1 \mathrm{tol} / \mathrm{MeOH}$<smiles>COC(=O)[C@]1(Cc2ccccc2)CNc2ccccc2C1</smiles>

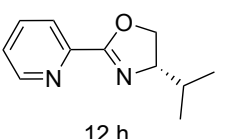

$88 \%$ yield, $52 \%$ ee

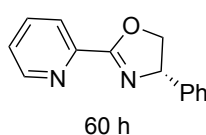

$38 \%$ yield, $2 \%$ ee

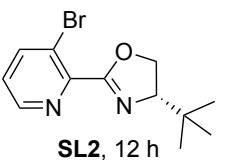

$87 \%$ yield, $89 \%$ ee

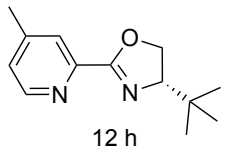

$91 \%$ yield, $83 \%$ ee

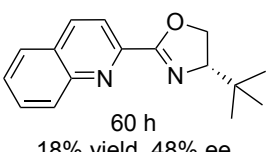

$18 \%$ yield, $48 \%$ ee

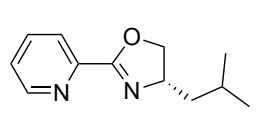

$15 \mathrm{~h}$ $55 \%$ yield, $61 \%$ ee<smiles>CC(C)(C)[C@H]1N=C(c2ccccn2)OC1(c1ccccc1)c1ccccc1</smiles>

$2 \%$ yield, $67 \%$ ee

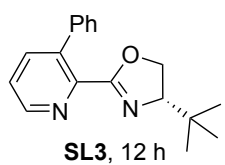

$88 \%$ yield, $52 \%$ ee

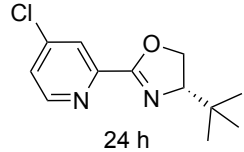

$73 \%$ yield, $85 \%$ ee

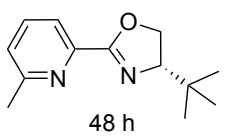

$10 \%$ yield, $37 \%$ ee

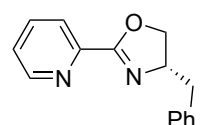

$5 \mathrm{~h}$ $75 \%$ yield, $60 \%$ ee

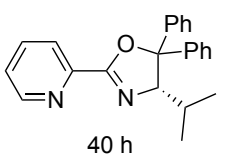

$30 \%$ yield, $16 \%$ ee

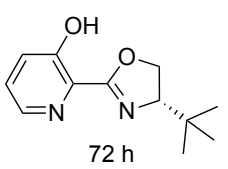

$20 \%$ yield, $11 \%$ ee

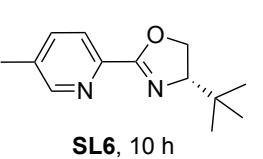

\% yield, $86 \%$ ee

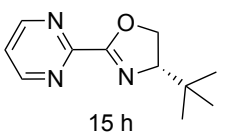

$44 \%$ yield, $73 \%$ ee

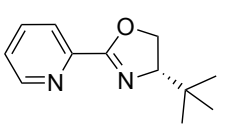

$15 \mathrm{~h}$ $87 \%$ yield, $87 \%$ ee

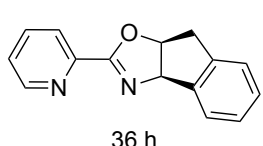

$76 \%$ yield, $18 \%$ ee

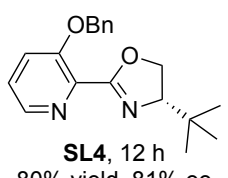

$80 \%$ yield, $81 \%$ ee

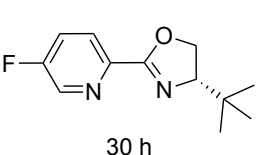

$83 \%$ yield, $85 \%$ ee

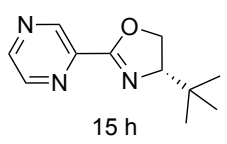

$48 \%$ yield, $57 \%$ ee

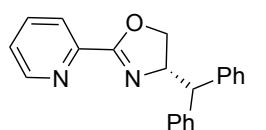

$20 \mathrm{~h}$

$80 \%$ yield, $66 \%$ ee

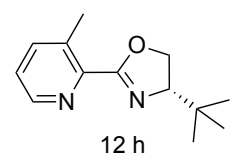

$86 \%$ yield, $82 \%$ ee

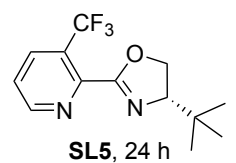

$60 \%$ yield, $85 \%$ ee

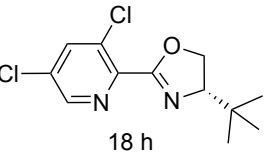

$52 \%$ yield, $84 \%$ ee

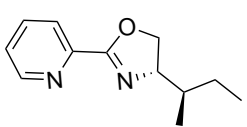

$12 \mathrm{~h}$

$90 \%$ yield, $55 \%$ ee

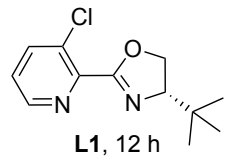

L1, $12 \mathrm{~h}$

$95 \%$ yield, $90 \%$ ee

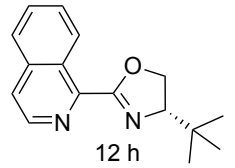

$87 \%$ yield, $81 \%$ ee

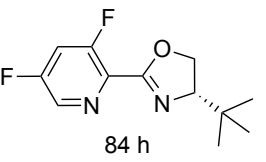




\section{Cu source screening}

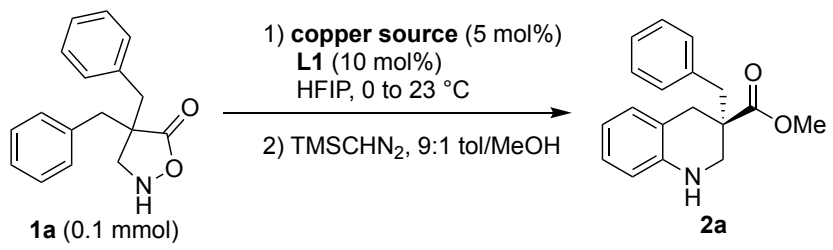

$\begin{array}{cccc}\mathrm{Cu}-\mathrm{Source} & \text { Time } & \text { Yield (\%) } & \text { ee \% } \\ \mathrm{CuOAc} & 12 \mathrm{~h} & 92 & 90 \\ \mathrm{CuCl} & 12 \mathrm{~h} & 82 & 92 \\ \mathrm{CuBr} & 12 \mathrm{~h} & 95 & 94 \\ \mathrm{MesCu} & 12 \mathrm{~h} & 88 & 89 \\ \mathrm{Cu}\left(\mathrm{CH}_{3} \mathrm{CN}\right)_{4} \cdot \mathrm{OTf} & 12 \mathrm{~h} & 82 & 88 \\ \mathrm{Cu}\left(\mathrm{CH}_{3} \mathrm{CN}\right)_{4} \cdot \mathrm{PF}_{6} & 12 \mathrm{~h} & 53 & 86\end{array}$

\section{Optimization of the metal:ligand ratio}

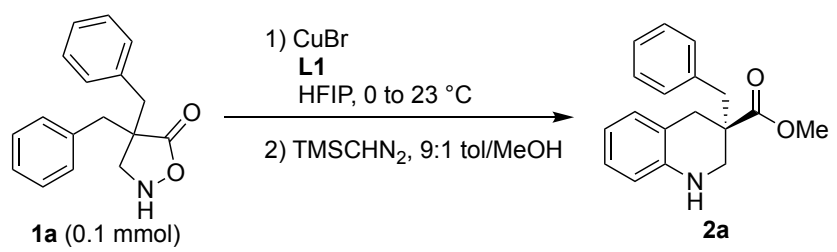

$\begin{array}{ccccc}\mathrm{CuBr}(\mathrm{mol} \%) & \text { L1 }(\mathrm{mol} \%) & \text { Time } & \text { Yield }(\%) & \text { ee } \% \\ 5 \mathrm{~mol} \% & 10 \mathrm{~mol} \% & 2 \mathrm{~h} & 90 & 94 \\ 5 \mathrm{~mol} \% & 6 \mathrm{~mol} \% & 2 \mathrm{~h} & 90 & 92 \\ 10 \mathrm{~mol} \% & 12 \mathrm{~mol} \% & 2 \mathrm{~h} & 93 & 94 \\ 15 \mathrm{~mol} \% & 17 \mathrm{~mol} \% & 2 \mathrm{~h} & 93 & 95 \\ 2 \mathrm{~mol} \% & 3 \mathrm{~mol} \% & 6 \mathrm{~h} & 91 & 91\end{array}$

\section{Synthesis of substituted isoxazolidin-5-ones}

\section{3-1. Structure of substrate precursors}<smiles>O=C1OCCC1Cc1ccccc1</smiles>

s1<smiles>Cc1ccc(CC2CN(C(=O)O)C(=O)O2)cc1</smiles><smiles>O=C1CC(Cc2ccc(-c3ccccc3)cc2)C(=O)O1</smiles>

S2<smiles>CC(C)(C)c1ccc(CC2CN(C(=O)O)C(=O)O2)cc1</smiles><smiles>Cc1ccccc1CC1CN(C(C)(C)C)CC1=O</smiles>

S3<smiles>O=C1O[Nb+2]CC1Cc1ccc(Cl)cc1</smiles><smiles></smiles>

S4<smiles>O=C1ON(S(=O)(=O)c2ccccc2)CC1CC1CCCCC1</smiles><smiles>O=C1O[Nb]2CC(Cc3ccccc3Cl)C1O2</smiles>

S5<smiles>O=C1O[Nb]2CCCCC12</smiles>

All 4-substituted isoxazolidin-5-ones and disubstituted substrates 1a, 3a, 3b and 7a are known in the literature. ${ }^{3}$ 


\section{3-2. Substrate synthesis}

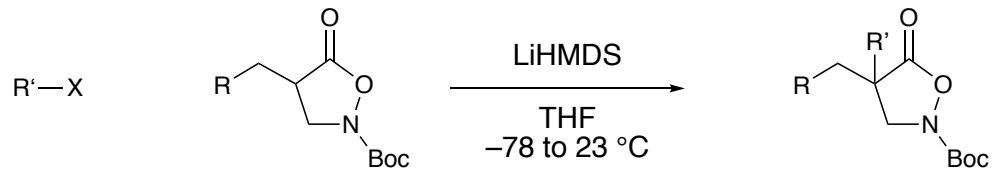

General procedure B: To a solution of Boc-protected isoxazolidine-5-one (1.0 equiv) in THF (0.3 M) was slowly added LiHMDS ( $1 \mathrm{M}$ in THF, 1.6 equiv) at $-78{ }^{\circ} \mathrm{C}$ and the resulting solution was stirred for $1 \mathrm{~h}$ at $-78{ }^{\circ} \mathrm{C}$. To the mixture, benzyl halide ( 2 equiv) was added at the same temperature, and the solution was gradually warmed to an ambient temperature. The reaction progress was monitored by TLC, and the reaction was quenched after completion by the addition of saturated aqueous $\mathrm{NH}_{4} \mathrm{Cl}$ solution. The aqueous phase was extracted with EtOAc (3x) and the organic phase was concentrated under reduced pressure. The obtained crude residue was purified by flash column chromatography, eluting with hexane/ethyl acetate to provide the alkylated product.

\section{tert-Butyl 4,4-bis(4-methylbenzyl)-5-oxoisoxazolidine-2-carboxylate (Boc-1b)}

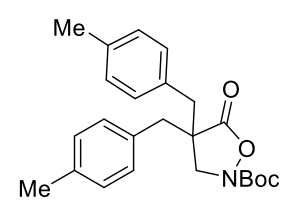

Prepared by the general procedure B from S2 $(1.0 \mathrm{~g}, 3.43 \mathrm{mmol})$ and 4-methylbenzyl bromide (1.26 g, $6.86 \mathrm{mmol})$, and isolated by silica gel column chromatgraphy (hexane/ethyl acetate) as a white solid (700 mg, 52\% yield). m.p. $141-143{ }^{\circ} \mathrm{C} ;{ }^{1} \mathbf{H}$ NMR (400 MHz, $\left.\mathrm{CDCl}_{3}\right)$ : $\delta$ 7.26$7.05(\mathrm{~m}, 8 \mathrm{H}), 3.90(\mathrm{~s}, 2 \mathrm{H}), 3.11(\mathrm{~d}, J=13.8 \mathrm{~Hz}, 2 \mathrm{H}), 2.77(\mathrm{~d}, J=13.9 \mathrm{~Hz}, 2 \mathrm{H}), 2.31(\mathrm{~s}, 6 \mathrm{H})$,

1.41 (s, 9H); ${ }^{13} \mathrm{C}$ NMR (101 MHz, $\left.\mathrm{CDCl}_{3}\right): \delta 176.2,155.2,137.1,131.8,130.3,129.4,83.6,52.7,51.12,40.4,28.0$, 21.0; IR (KBr): 3004, 2980, 2928, 1785, 1728, 1514, 1473, 1448, 1337, 1119, 846, $756 \mathrm{~cm}^{-1}$; HRMS (ESI) m/z calc'd for $\mathrm{C}_{24} \mathrm{H}_{29} \mathrm{O}_{4} \mathrm{NNa}[\mathrm{M}+\mathrm{Na}]^{+}: 418.1989$, found: 418.1988 .

\section{tert-Butyl 4,4-bis(2-methylbenzyl)-5-oxoisoxazolidine-2-carboxylate (Boc-1c)}

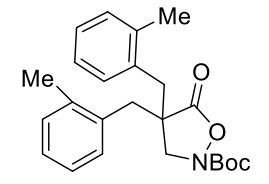

Prepared by the general procedure B from S3 $(1.0 \mathrm{~g}, 3.43 \mathrm{mmol})$ and 2-methylbenzyl bromide (919 $\mathrm{mg}, 6.86 \mathrm{mmol}$ ), and isolated by silica gel column chromatgraphy (hexane/ethyl acetate) as (m, 8H), 3.79 (s, 2H), 3.14 (d, $J=14.2 \mathrm{~Hz}, 2 \mathrm{H}), 3.02$ (d, $J=14.2 \mathrm{~Hz}, 2 \mathrm{H}), 2.18(\mathrm{~s}, 6 \mathrm{H}), 1.46(\mathrm{~s}, 9 \mathrm{H}) ;{ }^{13} \mathbf{C}$ NMR (101 MHz, $\left.\mathrm{CDCl}_{3}\right): \delta 176.3,155.3,137.1,133.6,130.8,130.7,127.6,126.5,83.9,52.7,51.6,36.1,28.1,19.9$; IR (KBr): 3004, 2980, 2928, 1785, 1725, 1514, 1448, 1366, 1337, 1227, 1154, 1036, 930, 846, 810, $756 \mathrm{~cm}^{-1}$; HRMS (ESI) $m / z$ calc'd for $\mathrm{C}_{24} \mathrm{H}_{29} \mathrm{O}_{4} \mathrm{NNa}[\mathrm{M}+\mathrm{Na}]^{+}: 418.1989$, found: 418.1988 .

\section{tert-Butyl 4,4-bis([1,1'-biphenyl]-4-ylmethyl)-5-oxoisoxazolidine-2-carboxylate (Boc-1d)}

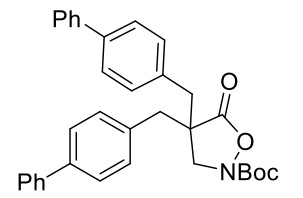

Prepared by the general procedure B from S6 (1.0 g, $2.83 \mathrm{mmol})$ and 4-phenylbenzyl bromide (1.39 g, $5.66 \mathrm{mmol})$, and isolated by silica gel column chromatgraphy (hexane/ethyl acetate) as a white solid (1.10 g, 74\% yield). m.p. $161-163{ }^{\circ} \mathrm{C} ;{ }^{1} \mathbf{H}$ NMR (400 MHz, $\left.\mathrm{CDCl}_{3}\right): \delta 7.59-$ 
$7.55(\mathrm{~m}, 8 \mathrm{H}), 7.46-7.42(\mathrm{~m}, 4 \mathrm{H}), 7.37-7.33(\mathrm{~m}, 2 \mathrm{H}), 7.28(\mathrm{~d}, J=8.2 \mathrm{~Hz}, 4 \mathrm{H}), 4.00(\mathrm{~s}, 2 \mathrm{H}), 3.23(\mathrm{~d}, J=13.8 \mathrm{~Hz}$, 2H), $2.91(\mathrm{~d}, J=13.8 \mathrm{~Hz}, 2 \mathrm{H}), 1.41(\mathrm{~s}, 9 \mathrm{H}) ;{ }^{13} \mathbf{C}$ NMR (101 MHz, $\left.\mathrm{CDCl}_{3}\right): \delta 176.1,155.5,150.3,131.8,130.2$, 128.8, 125.8, 125.6, 83.7, 52.9, 50.9, 39.9, 34.5, 31.3, 31.3, 28.1; IR (KBr): 3027, 2975, 2930, 1785, 1736, 1698, 1486, 1229, 1145, 849, $754 \mathrm{~cm}^{-1}$; HRMS (ESI) $\mathrm{m} / z$ calc'd for $\mathrm{C}_{34} \mathrm{H}_{33} \mathrm{O}_{4} \mathrm{~N} \mathrm{Na}[\mathrm{M}+\mathrm{Na}]^{+}: 542.2302$, found: 542.2301 .

\section{tert-Butyl 4,4-bis(4-(tert-butyl)benzyl)-5-oxoisoxazolidine-2-carboxylate (Boc-1e)}

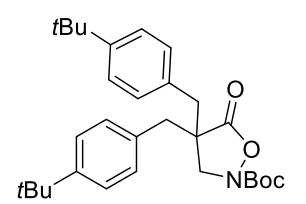

Prepared by the general procedure B from S7 $(1.0 \mathrm{~g}, 3.0 \mathrm{mmol})$ and 4-tert-butylbenzyl bromide $(1.10 \mathrm{~g}, 6.0 \mathrm{mmol})$, and isolated by silica gel column chromatgraphy (hexane/ethyl acetate) as a white solid (1.06 g, 74\% yield). m.p. $174-176{ }^{\circ} \mathrm{C} ;{ }^{1} \mathbf{H}$ NMR (400 $\left.\mathrm{MHz}, \mathrm{CDCl}_{3}\right)$ : $\delta 7.33-7.31(\mathrm{~m}, 4 \mathrm{H}), 7.12-7.10(\mathrm{~m}, 4 \mathrm{H}), 3.93(\mathrm{~s}, 2 \mathrm{H}), 3.10(\mathrm{~d}, J=13.9 \mathrm{~Hz}, 2 \mathrm{H}), 2.80(\mathrm{~d}, J=$ $13.9 \mathrm{~Hz}, 2 \mathrm{H}), 1.45$ (s, 9H), 1.30 (s, 18H); ${ }^{13} \mathbf{C} \mathbf{N M R}\left(101 \mathrm{MHz}, \mathrm{CDCl}_{3}\right): \delta 176.1,155.5,150.3,131.8,130.2,128.8$, 125.8, 125.6, 83.7, 52.9, 50.9, 39.9, 34.5, 31.3, 31.3, 28.1; IR (KBr): 3027, 2868, 1780, 1737, 1509, 1458, 1369, 1269, 1156, 1040, 847, 826, $757 \mathrm{~cm}^{-1}$; HRMS (ESI) $\mathrm{m} / z$ calc'd for $\mathrm{C}_{30} \mathrm{H}_{41} \mathrm{O}_{4} \mathrm{NNa}[\mathrm{M}+\mathrm{Na}]^{+}: 502.2928$, found: 502.2929 .

\section{tert-Butyl 4,4-bis(4-bromobenzyl)-5-oxoisoxazolidine-2-carboxylate (Boc-1f)}

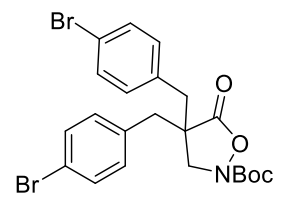

Prepared by the general procedure B from S4 (700 mg, $1.97 \mathrm{mmol})$ and 4-bromobenzyl bromide (985 mg, $3.94 \mathrm{mmol})$, and isolated as a white solid (390 g, 38\% yield). m.p. 189$191{ }^{\circ} \mathrm{C} ;{ }^{1} \mathbf{H}$ NMR $\left(400 \mathrm{MHz}, \mathrm{CDCl}_{3}\right): \delta 7.46-7.43(\mathrm{~m}, 4 \mathrm{H}), 7.06-7.03(\mathrm{~m}, 4 \mathrm{H}), 3.87(\mathrm{~s}, 2 \mathrm{H})$, $3.10(\mathrm{~d}, J=13.9 \mathrm{~Hz}, 2 \mathrm{H}), 2.76(\mathrm{~d}, J=13.9 \mathrm{~Hz}, 2 \mathrm{H}), 1.43(\mathrm{~s}, 9 \mathrm{H}) ;{ }^{13} \mathbf{C}$ NMR $\left(101 \mathrm{MHz}, \mathrm{CDCl}_{3}\right)$ : $\delta$ 175.4, 155.1, 133.5, 132.0, 121.9, 84.1, 52.6, 50.8, 40.2, 28.0; IR (KBr): 3001, 2979, 2934, 1786, 1735, 1488, 1367, 1333, 1154, 1012, 842, $758 \mathrm{~cm}^{-1}$; HRMS (ESI) $\mathrm{m} / z$ calc'd for $\mathrm{C}_{22} \mathrm{H}_{23} \mathrm{O}_{4} \mathrm{NBr}_{2} \mathrm{Na}[\mathrm{M}+\mathrm{Na}]^{+}: 545.9886$, found: 545.9885 .

\section{tert-Butyl 4,4-bis(2-chlorobenzyl)-5-oxoisoxazolidine-2-carboxylate (Boc-1g)}

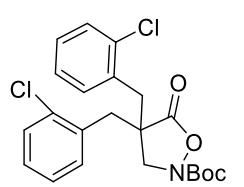

Prepared by the general procedure B from S5 $(1.42 \mathrm{~g}, 4.56 \mathrm{mmol})$ and 2-chlorobenzyl bromide (1.18 g, $9.13 \mathrm{mmol})$, and isolated by silica gel column chromatgraphy (hexane/ethyl acetate) as a white solid (1.45 g, 73\% yield). m.p. $119-121{ }^{\circ} \mathrm{C} ;{ }^{1} \mathbf{H}$ NMR (400 MHz, $\left.\mathrm{CDCl}_{3}\right): \delta 7.40-7.36(\mathrm{~m}$, 2H), $7.33-7.30(\mathrm{~m}, 2 \mathrm{H}), 7.24-7.20(\mathrm{~m}, 4 \mathrm{H}), 3.93(\mathrm{~s}, 2 \mathrm{H}), 3.36(\mathrm{~d}, J=14.0 \mathrm{~Hz}, 2 \mathrm{H}), 3.19$ (d, $J=$ $14.0 \mathrm{~Hz}, 2 \mathrm{H}), 1.44$ (s, 9H); ${ }^{13} \mathbf{C}$ NMR (101 MHz, $\left.\mathrm{CDCl}_{3}\right): \delta 175.3,155.1,135.2,133.0,132.2,129.8,129.1,127.3$, 83.8, 53.5, 51.6, 36.8, 28.1; IR (KBr): 3000, 2979, 2934, 1786, 1726, 1488, 1367, 1333, 1154, 1073, 1012, 842, $759 \mathrm{~cm}^{-1}$; HRMS (ESI) $\mathrm{m} / z$ calc'd for $\mathrm{C}_{22} \mathrm{H}_{23} \mathrm{O}_{4} \mathrm{NCl}_{2} \mathrm{Na}$ [M + Na $]^{+}:$458.0896, found: 458.0892 . 


\section{tert-Butyl 4-benzyl-4-(2-methylbenzyl)-5-oxoisoxazolidine-2-carboxylate (Boc-3c)}

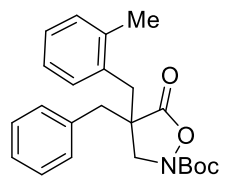

Prepared by the general procedure B from $\mathbf{S 1}(800 \mathrm{mg}, 2.88 \mathrm{mmol})$ and 2-methylbenzyl bromide (763 $\mathrm{mg}, 5.77 \mathrm{mmol}$ ), and isolated by silica gel column chromatgraphy (hexane/ethyl acetate) as a white solid (900 mg, 81\% yield). m.p. $94-96{ }^{\circ} \mathrm{C}$; ${ }^{1} \mathbf{H}$ NMR (400 MHz, $\left.\mathrm{CDCl}_{3}\right): \delta 7.36-7.27$ (m, 3H), 7.23-7.18 (m, 2H), $7.19-7.11(\mathrm{~m}, 4 \mathrm{H}), 4.02(\mathrm{~d}, J=11.2 \mathrm{~Hz}, 1 \mathrm{H}), 3.64(\mathrm{~d}, J=11.2 \mathrm{~Hz}, 1 \mathrm{H}), 3.15(\mathrm{dd}, J=$ 14.0, $1.7 \mathrm{~Hz}, 2 \mathrm{H}), 3.01$ (d, $J=14.3 \mathrm{~Hz}, 1 \mathrm{H}), 2.86(\mathrm{~d}, J=13.7 \mathrm{~Hz}, 1 \mathrm{H}), 2.10(\mathrm{~s}, 3 \mathrm{H}), 1.44(\mathrm{~s}, 9 \mathrm{H}) ;{ }^{13} \mathbf{C}$ NMR $(101$ $\left.\mathrm{MHz}, \mathrm{CDCl}_{3}\right): \delta 176.2,155.3,137.0,134.8,133.6,130.8,130.6,130.5,128.7,127.6,127.6,126.5,83.8,52.5,51.2$, 40.7, 36.0, 28.1, 19.8; IR (KBr): 3019, 2982, 2938, 1787, 1739, 1514, 1458, 1370, 1330, 1304, 1260, 1149, 1094 , 1024, 845, 786, $750 \mathrm{~cm}^{-1}$; HRMS (ESI) $\mathrm{m} / z$ calc'd for $\mathrm{C}_{23} \mathrm{H}_{27} \mathrm{O}_{4} \mathrm{NNa}[\mathrm{M}+\mathrm{Na}]^{+}:$404.1832, found: 404.1834.

\section{tert-Butyl 4-(2-methylbenzyl)-4-(4-methylbenzyl)-5-oxoisoxazolidine-2-carboxylate (Boc-3d)}

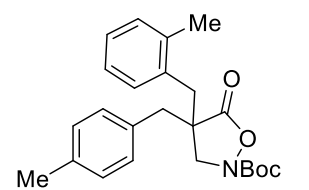

Prepared by the general procedure B from S3 $(700 \mathrm{mg}, 2.40 \mathrm{mmol})$ and 4-methylbenzyl bromide ( $888 \mathrm{mg}, 4.80 \mathrm{mmol}$ ), and isolated by silica gel column chromatgraphy (hexane/ethyl acetate) as a white solid (740 mg, 78\% yield). m.p. $111-112{ }^{\circ} \mathrm{C} ;{ }^{1} \mathbf{H}$ NMR (400 MHz, $\left.\mathrm{CDCl}_{3}\right)$ : $\delta 7.19-7.09(\mathrm{~m}, 8 \mathrm{H}), 4.02(\mathrm{~d}, J=11.1 \mathrm{~Hz}, 1 \mathrm{H}), 3.63(\mathrm{~d}, J=11.1 \mathrm{~Hz}, 1 \mathrm{H}), 3.12(\mathrm{t}, J=14.0 \mathrm{~Hz}, 2 \mathrm{H}), 3.00(\mathrm{~d}, J=$ $14.3 \mathrm{~Hz}, 1 \mathrm{H}), 2.82(\mathrm{~d}, J=13.7 \mathrm{~Hz}, 1 \mathrm{H}), 2.33(\mathrm{~s}, 3 \mathrm{H}), 2.11(\mathrm{~s}, 3 \mathrm{H}), 1.44(\mathrm{~s}, 9 \mathrm{H}) ;{ }^{13} \mathbf{C} \mathbf{N M R}\left(101 \mathrm{MHz}, \mathrm{CDCl}_{3}\right): \delta$ 176.3, 155.3, 137.2, 137.0, 133.7, 131.6, 130.8, 130.6, 130.4, 129.4, 127.5, 126.5, 83.7, 52.5, 51.3, 40.4, 36.0, 28.1, 21.1, 19.8; IR (KBr): 3019, 2982, 2938, 2877, 1786, 1738, 1514, 1458, 1370, 1330, 1304, 1260, 1150, 1094, 1024, 844, 806, 786, $750 \mathrm{~cm}^{-1}$; HRMS (ESI) $\mathrm{m} / z$ calc'd for $\mathrm{C}_{24} \mathrm{H}_{29} \mathrm{O}_{4} \mathrm{NNa}[\mathrm{M}+\mathrm{Na}]^{+}: 418.1989$, found: 418.1989 .

\section{tert-Butyl 4-(4-bromobenzyl)-4-(4-chlorobenzyl)-5-oxoisoxazolidine-2-carboxylate (Boc-3e)}

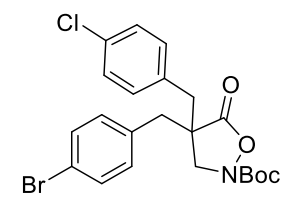

Prepared by the general procedure B from S4 (700 $\mathrm{mg}, 1.97 \mathrm{mmol})$ and 4-chlorobenzyl bromide ( $810 \mathrm{mg}, 3.94 \mathrm{mmol})$, and isolated by silica gel column chromatgraphy (hexane/ethyl

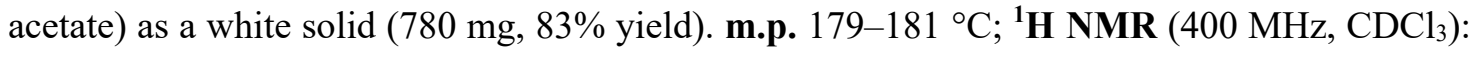
$\delta 7.46-7.43(\mathrm{~m}, 2 \mathrm{H}), 7.31-7.27(\mathrm{~m}, 2 \mathrm{H}), 7.12-7.09(\mathrm{~m}, 2 \mathrm{H}), 7.07-7.03(\mathrm{~m}, 2 \mathrm{H}), 3.87$ (s, 2H), $3.11(\mathrm{dd}, J=13.9,5.7 \mathrm{~Hz}, 2 \mathrm{H}), 2.77(\mathrm{dd}, J=13.9,5.3 \mathrm{~Hz}, 2 \mathrm{H}), 1.43(\mathrm{~s}, 9 \mathrm{H}) ;{ }^{13} \mathbf{C ~ N M R}\left(101 \mathrm{MHz}, \mathrm{CDCl}_{3}\right): \delta$ 175.4, 155.0, 133.8, 133.5, 133.0, 132.0, 131.6, 129.0, 121.9, 84.1, 52.6, 50.9, 40.2, 40.2, 28.0; IR (KBr): 3002, 2980, 2935, 1787, 1726, 1590, 1488, 1367, 1334, 1260, 1155, 1092, 1013, 933, 843, 817, $758 \mathrm{~cm}^{-1}$; HRMS (ESI) $m / z$ calc'd for $\mathrm{C}_{22} \mathrm{H}_{23} \mathrm{O}_{4} \mathrm{NBrClNa}[\mathrm{M}+\mathrm{Na}]^{+}:$502.0391, found: 502.0388 .

\section{tert-Butyl 4-(cyclohexylmethyl)-4-(4-methylbenzyl)-5-oxoisoxazolidine-2-carboxylate (Boc-7b)}

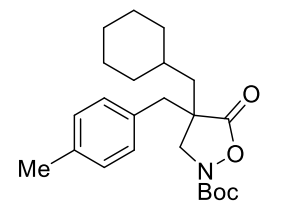

Prepared by the general procedure B from S9 $(750 \mathrm{mg}, 2.65 \mathrm{mmol})$ and 4-methylbenzyl bromide $(980 \mathrm{mg}, 5.29 \mathrm{mmol})$, and isolated by silica gel column chromatgraphy (hexane/ethyl acetate) as a white solid (710 mg, 69\% yield). m.p. $107-109{ }^{\circ} \mathrm{C} ;{ }^{1} \mathbf{H}$ NMR (400 MHz, $\left.\mathrm{CDCl}_{3}\right)$ : $\delta$ 7.12-7.10 (m, 2H), 7.05-7.03 (m, 2H), $4.01(\mathrm{~d}, J=11.1 \mathrm{~Hz}, 1 \mathrm{H}), 3.86(\mathrm{~d}, J=11.1 \mathrm{~Hz}, 1 \mathrm{H})$, 
$3.00(\mathrm{~d}, J=13.8 \mathrm{~Hz}, 1 \mathrm{H}), 2.75(\mathrm{~d}, J=13.8 \mathrm{~Hz}, 1 \mathrm{H}), 2.31(\mathrm{~s}, 3 \mathrm{H}), 1.71-1.54(\mathrm{~m}, 8 \mathrm{H}), 1.47$ (s, 9H), 1.13-1.10 (m, $3 \mathrm{H}), 1.02-0.92(\mathrm{~m}, 2 \mathrm{H}) ;{ }^{13} \mathbf{C}$ NMR $\left(101 \mathrm{MHz}, \mathrm{CDCl}_{3}\right): \delta 176.9,155.6,137.0,132.0,130.2,129.4,83.6,54.8$, 49.0, 42.1, 40.8, 35.0, 34.0, 33.8, 28.1, 26.2, 26.2, 26.0, 21.0; IR (KBr): 2979, 2924, 2852, 1797, 1736, 1516, 1446, 1369, 1308, 1304, 1224, 1142, 1078, 1011, 845, 806, $770 \mathrm{~cm}^{-1}$; HRMS (ESI) $m / z$ calc'd for $\mathrm{C}_{23} \mathrm{H}_{34} \mathrm{O}_{4} \mathrm{~N}[\mathrm{M}+\mathrm{H}]^{+}$: 388.2482, found: 388.2483 .

tert-Butyl 4-(cyclohexylmethyl)-4-(2-methylbenzyl)-5-oxoisoxazolidine-2-carboxylate (Boc-7c)

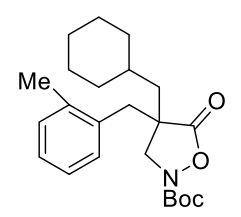

Prepared by the general procedure B from S9 $(750 \mathrm{mg}, 2.65 \mathrm{mmol})$ and 2-methylbenzyl bromide (700 $\mu 1,5.3 \mathrm{mmol})$, and isolated by silica gel column chromatgraphy (hexane/ethyl acetate) as a white solid (830 mg, 80\% yield). m.p. $73-75^{\circ} \mathrm{C} ;{ }^{1} \mathbf{H}$ NMR (400 MHz, $\mathrm{CDCl}_{3}$ ): $\delta 7.16-7.14(\mathrm{~m}$, 4H), $3.95(\mathrm{~d}, J=11.0 \mathrm{~Hz}, 1 \mathrm{H}), 3.79(\mathrm{~d}, J=11.0 \mathrm{~Hz}, 1 \mathrm{H}), 3.08$ (d, $J=14.3 \mathrm{~Hz}, 1 \mathrm{H}), 2.95$ (d, $J=$ $14.3 \mathrm{~Hz}, 1 \mathrm{H}), 2.29$ (s, 3H), $1.74-1.59$ (m, 8H), 1.47 (s, 9H), $1.31-1.11(\mathrm{~m}, 3 \mathrm{H}), 1.09-0.96(\mathrm{~m}, 2 \mathrm{H}) ;{ }^{13} \mathbf{C}$ NMR (101 MHz, $\left.\mathrm{CDCl}_{3}\right): \delta 176.9,155.6,136.9,133.9,130.8,130.5,127.5,126.4,83.7,54.6,49.4,42.7,36.8,35.1,34.1$, 34.1, 28.1, 26.2, 26.2, 26.0, 20.1; IR (KBr): 2979, 2923, 2852, 1798, 1736, 1516, 1446, 1369, 1308, 1304, 1224, 1143, 1078, 1011, 845, 806, $770 \mathrm{~cm}^{-1}$; HRMS (ESI) $\mathrm{m} / z$ calc'd for $\mathrm{C}_{23} \mathrm{H}_{34} \mathrm{O}_{4} \mathrm{~N}[\mathrm{M}+\mathrm{H}]^{+}: 388.2482$, found: 388.2484 .

\section{tert-Butyl 4-([1,1'-biphenyl]-4-ylmethyl)-4-(cyclohexylmethyl)-5-oxoisoxazolidine-2-carboxylate (Boc-7d)}

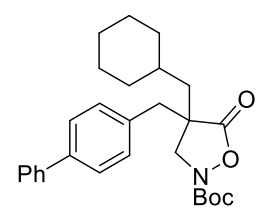

Prepared by the general procedure B from $\mathbf{S 9}(600 \mathrm{mg}, 2.22 \mathrm{mmol})$ and 4-phenylbenzyl bromide (980 mg, $5.29 \mathrm{mmol})$, and isolated by silica gel column chromatgraphy (hexane/ethyl acetate) as a white solid (640 mg, 67\% yield). m.p. $109-111^{\circ} \mathrm{C} ;{ }^{1} \mathbf{H}$ NMR (400 $\left.\mathrm{MHz}, \mathrm{CDCl}_{3}\right): \delta 7.58-$ $7.52(\mathrm{~m}, 4 \mathrm{H}), 7.46-7.41(\mathrm{~m}, 2 \mathrm{H}), 7.36-7.32(\mathrm{~m}, 1 \mathrm{H}), 7.25-7.23(\mathrm{~m}, 2 \mathrm{H}), 4.05(\mathrm{~d}, J=11.1 \mathrm{~Hz}$,

1H), $3.91(\mathrm{~d}, J=11.1 \mathrm{~Hz}, 1 \mathrm{H}), 3.08(\mathrm{~d}, J=13.8 \mathrm{~Hz}, 1 \mathrm{H}), 2.84(\mathrm{~d}, J=13.8 \mathrm{~Hz}, 1 \mathrm{H}), 1.75-1.59(\mathrm{~m}, 7 \mathrm{H}), 1.46(\mathrm{~s}$, 9H), 1.31-1.11 (m, 4H), $1.05-0.95$ (m, 2H); ${ }^{13} \mathbf{C}$ NMR (101 MHz, $\left.\mathrm{CDCl}_{3}\right): \delta 176.8,155.6,140.5,140.3,134.1$, 130.7, 128.8, 127.4, 127.3, 127.0, 83.8, 54.9, 49.0, 42.1, 40.8, 35.0, 34.0, 33.9, 28.1, 26.2, 26.2, 26.0; IR (KBr): 2930, 2849, 1787, 1737, 1717, 1486, 1449, 1367, 1318, 1145, 1071, 847, $765 \mathrm{~cm}^{-1}$; HRMS (ESI) $\mathrm{m} / z$ calc'd for $\mathrm{C}_{28} \mathrm{H}_{35} \mathrm{O}_{4} \mathrm{NNa}[\mathrm{M}+\mathrm{Na}]^{+}: 472.2458$, found: 472.2455 .

\section{tert-Butyl 4-benzyl-5-oxo-4-((tetrahydro-2H-pyran-4-yl)methyl)isoxazolidine-2-carboxylate (Boc-7e)}

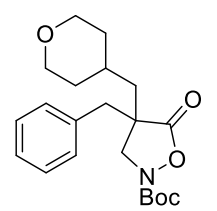

Prepared by the general procedure B from $\mathbf{S 1 0}(1.3 \mathrm{~g}, 4.55 \mathrm{mmol})$ and benzyl bromide $(1082 \mathrm{mg}$, $9.1 \mathrm{mmol}$ ), and isolated by silica gel column chromatgraphy (hexane/ethyl acetate) as a white solid (927 mg, 52\% yield). m.p. $85-87{ }^{\circ} \mathrm{C} ;{ }^{1} \mathbf{H}$ NMR (400 MHz, $\left.\mathrm{CDCl}_{3}\right): \delta 7.34-7.27$ (m, 3H), $7.17-$ $7.15(\mathrm{~m}, 2 \mathrm{H}), 4.02(\mathrm{~d}, J=11.1 \mathrm{~Hz}, 1 \mathrm{H}), 3.93-3.89(\mathrm{~m}, 2 \mathrm{H}), 3.85(\mathrm{~d}, J=11.1 \mathrm{~Hz}, 1 \mathrm{H}), 3.38$ (tdd, $J$ $=11.8,4.0,2.3 \mathrm{~Hz}, 2 \mathrm{H}), 3.08(\mathrm{~d}, J=13.8 \mathrm{~Hz}, 1 \mathrm{H}), 2.82(\mathrm{~d}, J=13.8 \mathrm{~Hz}, 1 \mathrm{H}), 1.69-1.63(\mathrm{~m}, 2 \mathrm{H}), 1.61-1.55(\mathrm{~m}$, $3 \mathrm{H}), 1.47$ (s, 9H), 1.40-1.31 (m, 2H); ${ }^{13} \mathbf{C}$ NMR (101 MHz, $\left.\mathrm{CDCl}_{3}\right): \delta 176.5,155.6,134.8,130.2,128.8,127.6$, 
84.0, 67.7, 67.7, 55.3, 48.7, 41.8, 41.2, 34.4, 33.7, 31.4, 28.1; IR (KBr): 2956, 2936, 2837, 1783, 1737, 1604, 1497 , 1446, 1370, 1300, 1240, 1149, 1122, 1067, 847, $761 \mathrm{~cm}^{-1}$; HRMS (ESI) $m / z$ calc'd for $\mathrm{C}_{21} \mathrm{H}_{30} \mathrm{O}_{5} \mathrm{~N}[\mathrm{M}+\mathrm{H}]^{+}$: 376.2118, found: 376.2119 .

\section{tert-Butyl 4-benzyl-4-(4-nitrobenzyl)-5-oxoisoxazolidine-2-carboxylate (Boc-7f)}

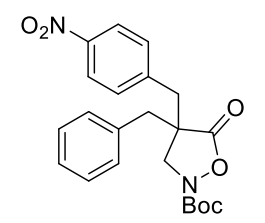

Prepared by the general procedure B from S1 (600 g, $2.16 \mathrm{mmol})$ and 4-nitrobenzyl bromide (933 $\mathrm{mg}, 4.32 \mathrm{mmol}$ ), and isolated by silica gel column chromatgraphy (hexane/ethyl acetate) as a white solid (450 mg, 51\% yield). m.p. $134-136{ }^{\circ} \mathrm{C} ;{ }^{1} \mathbf{H}$ NMR (400 MHz, $\left.\mathrm{CDCl}_{3}\right): \delta 8.18-8.16$ (m, 2H), $7.36-7.33(\mathrm{~m}, 5 \mathrm{H}), 7.20-7.18(\mathrm{~m}, 2 \mathrm{H}), 4.02(\mathrm{~d}, J=11.3 \mathrm{~Hz}, 1 \mathrm{H}), 3.79$ (d, $J=11.3 \mathrm{~Hz}$,

1H), $3.26(\mathrm{~d}, J=13.7 \mathrm{~Hz}, 1 \mathrm{H}), 3.17(\mathrm{~d}, J=13.8 \mathrm{~Hz}, 1 \mathrm{H}), 2.90(\mathrm{dd}, J=18.7,13.8 \mathrm{~Hz}, 2 \mathrm{H}), 1.42(\mathrm{~s}, 9 \mathrm{H}) ;{ }^{13} \mathbf{C} \mathbf{N M R}$ $\left(101 \mathrm{MHz}, \mathrm{CDCl}_{3}\right): \delta 175.2,155.1,147.5,142.4,134.2,131.3,130.4,128.9,127.9,123.9,84.2,53.0,51.0,40.9$, 40.2, 28.0; IR (KBr): 3007, 2980, 2923, 1786, 1722, 1602, 1522, 1455, 1348, 1229, 1152, 1031, 876, 839, 711, $648 \mathrm{~cm}^{-1}$; HRMS (ESI) $\mathrm{m} / z$ calc'd for $\mathrm{C}_{22} \mathrm{H}_{24} \mathrm{O}_{6} \mathrm{~N}_{2} \mathrm{Na}[\mathrm{M}+\mathrm{Na}]^{+}: 435.1527$, found: 435.1526.

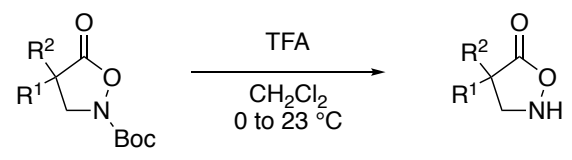

General procedure $\mathbf{C}^{3}$ To a solution of Boc-protected isoxazolidin-5-ones in $\mathrm{CH}_{2} \mathrm{Cl}_{2}(0.1 \mathrm{M})$ was added TFA $(0.2$ M) at $0{ }^{\circ} \mathrm{C}$, and the resulting mixture was stirred at room temperature for $1 \mathrm{~h}$. The reaction mixture was concentrated under reduced pressure. The resulting residue was diluted with EtOAc, followed by the addition of sat aq $\mathrm{NaHCO}_{3}$. The aqueous phase was extracted with EtOAc (3x). The combined organic layers were washed with brine, dried over $\mathrm{Na}_{2} \mathrm{SO}_{4}$, filtered, and concentrated under reduced pressure to give the crude product, which was purified by silica gel column chromatography to afford the corresponding unprotected isoxazolidin-5-ones.

\section{4,4-Bis(4-methylbenzyl)isoxazolidin-5-one (1b)}

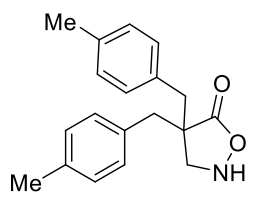

Prepared by the general procedure C from Boc-1b $(500 \mathrm{mg}, 1.69 \mathrm{mmol})$ and isolated by silica gel column chromatgraphy (hexane/ethyl acetate) as a white solid (300 mg, 81\% yield). m.p. 155$157^{\circ} \mathrm{C} ;{ }^{1} \mathbf{H}$ NMR (400 MHz, $\mathrm{CDCl}_{3}$ ): $7.14-7.08$ (m, 8H), 4.11 (br, 1H), 3.54 (s, 2H), 3.13 (d, J $=13.7 \mathrm{~Hz}, 2 \mathrm{H}), 2.81(\mathrm{~d}, J=13.7 \mathrm{~Hz}, 2 \mathrm{H}), 2.33(\mathrm{~s}, 6 \mathrm{H}) ;{ }^{13} \mathbf{C} \mathbf{N M R}\left(101 \mathrm{MHz}, \mathrm{CDCl}_{3}\right): \delta 181.2$, 137.5, 132.4, 129.7, 129.5, 54.7, 50.6, 42.2, 21.1; IR (KBr): 3248, 3018, 2919, 1757, 1513, 1448, 1215, 1169, 1107 , $1019,868,808,725 \mathrm{~cm}^{-1}$; HRMS (ESI) $m / z$ calc'd for $\mathrm{C}_{19} \mathrm{H}_{21} \mathrm{O}_{2} \mathrm{~N} \mathrm{Na}[\mathrm{M}+\mathrm{Na}]^{+}: 318.1465$, found: 318.1469 . 


\section{4,4-Bis(2-methylbenzyl)isoxazolidin-5-one (1c)}

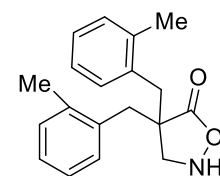

Prepared by the general procedure C from Boc-1c $(800 \mathrm{mg}, 2.02 \mathrm{mmol})$ and isolated by silica gel column chromatgraphy (hexane/ethyl acetate) as a white solid (480 mg, 80\% yield). m.p. 101$103{ }^{\circ} \mathrm{C} ;{ }^{1} \mathbf{H}$ NMR $\left(400 \mathrm{MHz}, \mathrm{CDCl}_{3}\right): \delta 7.21-7.14(\mathrm{~m}, 8 \mathrm{H}), 3.48(\mathrm{~s}, 2 \mathrm{H}), 3.18(\mathrm{~d}, J=14.1 \mathrm{~Hz}$, 2H), $3.06(\mathrm{~d}, J=14.1 \mathrm{~Hz}, 2 \mathrm{H}), 2.29$ (s, 6H); ${ }^{13} \mathbf{C}$ NMR (101 MHz, $\left.\mathrm{CDCl}_{3}\right): \delta 181.7,136.8,134.2,131.1,129.9$, 127.7, 126.7, 54.5, 51.0, 38.3, 20.0; IR (KBr): 3248, 3018, 2919, 1757, 1513, 1449, 1255, 1169, 1107, 1019, 868, $808,724 \mathrm{~cm}^{-1}$; HRMS (ESI) $m / z$ calc'd for $\mathrm{C}_{19} \mathrm{H}_{21} \mathrm{O}_{2} \mathrm{NNa}[\mathrm{M}+\mathrm{Na}]^{+}: 318.1465$, found: 318.1464 .

\section{4,4-Bis([1,1'-biphenyl]-4-ylmethyl)isoxazolidin-5-one (1d)}

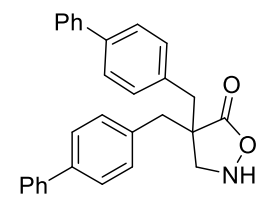

Prepared by the general procedure C from Boc-1d $(600 \mathrm{mg}, 1.15 \mathrm{mmol})$ and isolated by silica gel column chromatgraphy (hexane/ethyl acetate) as a white solid (250 mg, 51\% yield). m.p. 209-211 ${ }^{\circ} \mathrm{C} ;{ }^{1} \mathbf{H}$ NMR $\left(400 \mathrm{MHz}, \mathrm{CDCl}_{3}\right): \delta 7.59-7.56(\mathrm{~m}, 8 \mathrm{H}), 7.46-7.42(\mathrm{~m}, 4 \mathrm{H}), 7.37$ $7.30(\mathrm{~m}, 6 \mathrm{H}), 5.32(\mathrm{br}, 1 \mathrm{H}), 3.64(\mathrm{br}, 2 \mathrm{H}), 3.26(\mathrm{~d}, J=13.6 \mathrm{~Hz}, 2 \mathrm{H}), 2.94(\mathrm{~d}, J=9.0 \mathrm{~Hz}, 2 \mathrm{H})$;

${ }^{13}$ C NMR (101 MHz, $\left.\mathrm{CDCl}_{3}\right): \delta 181.1,140.3,134.6,130.1,128.9,127.7,127.5,127.0,54.8,50.8,42.2$; IR (KBr): 3239, 3027, 2854, 1769, 1598, 1485, 1448, 1364, 1254, 1171, 1027, 867, 828, $766 \mathrm{~cm}^{-1}$; HRMS (ESI) m/z calc'd for $\mathrm{C}_{29} \mathrm{H}_{25} \mathrm{O}_{2} \mathrm{NNa}[\mathrm{M}+\mathrm{Na}]^{+}$: 442.1778, found: 442.1777 .

\section{4,4-Bis(4-(tert-butyl)benzyl)isoxazolidin-5-one (1e)}

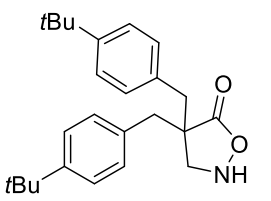

Prepared by the general procedure C from Boc-1e $(750 \mathrm{mg}, 1.56 \mathrm{mmol})$ and isolated by silica gel column chromatgraphy (hexane/ethyl acetate) as a white solid (310 mg, 52\% yield). m.p. 234-236 ${ }^{\circ} \mathrm{C} ;{ }^{1} \mathbf{H}$ NMR $\left(400 \mathrm{MHz}, \mathrm{CDCl}_{3}\right): \delta 7.35$ - $7.33(\mathrm{~m}, 4 \mathrm{H}), 7.16$ - $7.14(\mathrm{~m}, 4 \mathrm{H}), 5.12$ (br, 1H), 3.59 (br, 2H), $3.16(\mathrm{~d}, J=13.6 \mathrm{~Hz}, 2 \mathrm{H}), 2.82(\mathrm{~d}, J=10.7 \mathrm{~Hz}, 2 \mathrm{H}), 1.31(\mathrm{~s}, 18 \mathrm{H}) ;{ }^{13} \mathbf{C}$ NMR (101 MHz, $\left.\mathrm{CDCl}_{3}\right): \delta 181.4,150.8,132.5,129.4,125.9,54.7,50.6,42.2,34.5,31.3 ; \mathrm{IR}(\mathrm{KBr}): 3248,2965,2868$, 1766, 1508, 1446, 1362, 1108, 1021, 867, $823 \mathrm{~cm}^{-1}$; HRMS (ESI) $\mathrm{m} / z$ calc'd for $\mathrm{C}_{25} \mathrm{H}_{33} \mathrm{O}_{2} \mathrm{NNa}[\mathrm{M}+\mathrm{Na}]^{+}$: 402.2404, found: 402.2403 .

\section{4,4-Bis(4-bromobenzyl)isoxazolidin-5-one (1f)}

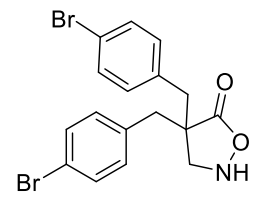

Prepared by the general procedure C from Boc-1f $(250 \mathrm{mg}, 0.47 \mathrm{mmol})$ and isolated by silica gel column chromatgraphy (hexane/ethyl acetate) as a white solid (145 mg, 71\% yield). m.p. 147$149{ }^{\circ} \mathrm{C} ;{ }^{1} \mathbf{H}$ NMR $\left(400 \mathrm{MHz}, \mathrm{CDCl}_{3}\right): \delta 7.46(\mathrm{~d}, J=8.1 \mathrm{~Hz}, 4 \mathrm{H}), 7.09(\mathrm{~d}, J=8.0 \mathrm{~Hz}, 4 \mathrm{H}), 5.38$ (br, 1H), 3.50 (br, 2H), $3.12(\mathrm{~d}, J=13.7 \mathrm{~Hz}, 2 \mathrm{H}), 2.79(\mathrm{~d}, J=11.3 \mathrm{~Hz}, 2 \mathrm{H}) ;{ }^{13} \mathbf{C}$ NMR $(101 \mathrm{MHz}$, $\left.\mathrm{CDCl}_{3}\right): \delta 180.2,134.3,132.2,131.4,122.0,54.2$, 50.4, 41.6; IR (KBr): 3243, 2956, 2928, 1758, 1590, 1487, 1449, 1407, 1252, 1169, 1073, 1013, 868, 812, $723 \mathrm{~cm}^{-1}$; HRMS (ESI) $\mathrm{m} / z$ calc'd for $\mathrm{C}_{17} \mathrm{H}_{15} \mathrm{O}_{2} \mathrm{NBr}_{2} \mathrm{Na}[\mathrm{M}+\mathrm{Na}]^{+}$: 445.9362, found: 445.9368 . 


\section{4,4-Bis(2-chlorobenzyl)isoxazolidin-5-one (1g)}

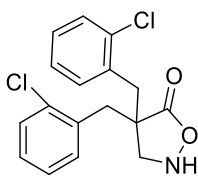

Prepared by the general procedure C from Boc-1g (1.45 g, $3.33 \mathrm{mmol})$ and isolated by silica gel column chromatgraphy (hexane/ethyl acetate) as a white solid (1.04 g, 93\% yield). m.p. $69-71{ }^{\circ} \mathrm{C}$;

${ }^{1} \mathbf{H}$ NMR (400 MHz, $\left.\mathrm{CDCl}_{3}\right): \delta 7.42-7.38(\mathrm{~m}, 2 \mathrm{H}), 7.36-7.33(\mathrm{~m}, 2 \mathrm{H}), 7.27-7.23(\mathrm{~m}, 4 \mathrm{H}), 3.95$ (br, 1H), 3.57 (s, 2H), $3.41(\mathrm{~d}, J=13.9 \mathrm{~Hz}, 2 \mathrm{H}), 3.22(\mathrm{~d}, J=13.9 \mathrm{~Hz}, 2 \mathrm{H}) ;{ }^{13} \mathbf{C} \mathbf{N M R}\left(101 \mathrm{MHz}, \mathrm{CDCl}_{3}\right): \delta 180.5$, 134.8, 133.6, 131.9, 130.0, 129.2, 127.6, 53.7, 51.5, 38.0; IR (KBr): 3243, 2956, 2928, 1758, 1590, 1487, 1407, $1252,1169,1073,1013,868,812,723 \mathrm{~cm}^{-1}$; HRMS (ESI) $\mathrm{m} / z$ calc'd for $\mathrm{C}_{17} \mathrm{H}_{16} \mathrm{O}_{2} \mathrm{NCl}_{2}[\mathrm{M}+\mathrm{H}]^{+}: 336.0553$, found: 336.0557 .

\section{4-Benzyl-4-(2-methylbenzyl)isoxazolidin-5-one (3c)}

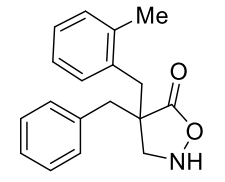

Prepared by the general procedure $\mathrm{C}$ from Boc-3c $(823 \mathrm{mg}, 2.15 \mathrm{mmol})$, and isolated by silica gel column chromatgraphy (hexane/ethyl acetate) as a white solid (542 mg, 89\% yield). m.p. 104$106{ }^{\circ} \mathrm{C} ;{ }^{1} \mathbf{H}$ NMR (400 MHz, $\left.\mathrm{CDCl}_{3}\right): \delta 7.37$ - 7.31 (m, 3H), 7.25 - $7.23(\mathrm{~m}, 2 \mathrm{H}), 7.20$ - 7.13 (m, 4H), 4.84 (br, 1H), 3.58 (d, $J=12.0 \mathrm{~Hz}, 1 \mathrm{H}), 3.46$ (d, $J=12.0 \mathrm{~Hz}, 1 \mathrm{H}), 3.19$ (dd, $J=16.3,13.9 \mathrm{~Hz}, 2 \mathrm{H}), 3.05$ (d, $J$ $=14.2 \mathrm{~Hz}, 1 \mathrm{H}), 2.88(\mathrm{~d}, J=13.6 \mathrm{~Hz}, 1 \mathrm{H}), 2.25(\mathrm{~s}, 3 \mathrm{H}) ;{ }^{13} \mathbf{C} \mathbf{~ N M R}\left(101 \mathrm{MHz}, \mathrm{CDCl}_{3}\right): \delta 181.3,136.7,135.6,134.1$, 131.1, 129.9, 129.6, 129.1, 127.8, 127.7, 126.7, 54.4, 50.7, 42.7, 38.1, 20.0; IR (KBr): 3238, 3024, 2957, 2925, $1771,1600,1493,1453,1361,256,1192,1107,1055,867,746 \mathrm{~cm}^{-1}$; HRMS (ESI) $\mathrm{m} / z$ calc'd for $\mathrm{C}_{18} \mathrm{H}_{19} \mathrm{O}_{2} \mathrm{NNa}$ $[\mathrm{M}+\mathrm{Na}]^{+}: 304.1308$, found: 304.1312 .

\section{4-(2-Methylbenzyl)-4-(4-methylbenzyl)isoxazolidin-5-one (3d)}

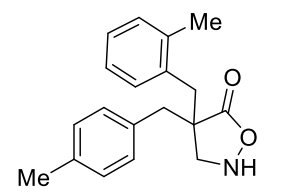

Prepared by the general procedure C from Boc-3d (740 $\mathrm{mg}, 1.87 \mathrm{mmol})$, and isolated by silica gel column chromatgraphy (hexane/ethyl acetate) as a white solid (451 mg, 81\% yield). m.p. 49-51 ${ }^{\circ} \mathrm{C} ;{ }^{1} \mathbf{H}$ NMR $\left(400 \mathrm{MHz}, \mathrm{CDCl}_{3}\right): \delta 7.20-7.15(\mathrm{~m}, 4 \mathrm{H}), 7.14-7.11(\mathrm{~m}, 4 \mathrm{H}), 5.20(\mathrm{br}$, 1H), 3.48 (br, 2H), 3.16 (dd, $J=13.9,4.1 \mathrm{~Hz}, 2 \mathrm{H}), 3.04$ (d, $J=14.2 \mathrm{~Hz}, 1 \mathrm{H}), 2.83(\mathrm{br}, 1 \mathrm{H}), 2.33$ (s, 3H), 2.26 (s, $3 \mathrm{H}) ;{ }^{13} \mathrm{C}$ NMR $\left(101 \mathrm{MHz}, \mathrm{CDCl}_{3}\right): \delta 181.5,136.8,134.3,132.5,131.0,130.0,129.8,127.6,126.7,54.6,50.8,42.4$, 38.2, 21.1, 20.1; IR (KBr): 3238, 3024, 2957, 2925, 1771, 1580, 1493, 1453, 1169, 1107, 1026, 928, 867, 762,705 $\mathrm{cm}^{-1}$; HRMS (ESI) $m / z$ calc'd for $\mathrm{C}_{19} \mathrm{H}_{21} \mathrm{O}_{2} \mathrm{NNa}[\mathrm{M}+\mathrm{Na}]^{+}: 318.1465$, found: 318.1467.

\section{4-(4-Bromobenzyl)-4-(4-chlorobenzyl)isoxazolidin-5-one (3e)}

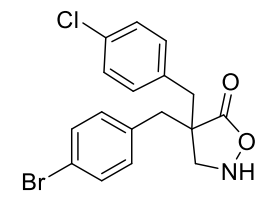

Prepared by the general procedure C from Boc-3e (795 mg, $1.65 \mathrm{mmol})$, and isolated by silica gel column chromatgraphy (hexane/ethyl acetate) as a white solid (524 mg, 83\% yield). m.p. 151-153 ${ }^{\circ} \mathrm{C} ;{ }^{1} \mathbf{H}$ NMR $\left(400 \mathrm{MHz}, \mathrm{CDCl}_{3}\right): \delta 7.48-7.45(\mathrm{~m}, 2 \mathrm{H}), 7.33-7.29(\mathrm{~m}, 2 \mathrm{H}), 7.16-$ $7.13(\mathrm{~m}, 2 \mathrm{H}), 7.10-7.07(\mathrm{~m}, 2 \mathrm{H}), 3.51(\mathrm{br}, 2 \mathrm{H}), 3.13(\mathrm{dd}, J=13.7,6.2 \mathrm{~Hz}, 2 \mathrm{H}), 2.80$ (dd, $J=$ 13.4, $3.7 \mathrm{~Hz}, 2 \mathrm{H}) ;{ }^{13} \mathbf{C}$ NMR (101 MHz, $\left.\mathrm{CDCl}_{3}\right): \delta 180.2,134.3,133.8,132.2,131.4,131.0,129.2,121.9,54.3$, 50.5, 41.6, 41.5; IR (KBr): 3244, 3021, 2956, 2929, 1912, 1758, 1591, 1489, 1449, 1409, 1253, 1169, 1094, 1015, $868,813,725 \mathrm{~cm}^{-1}$; HRMS (ESI) $\mathrm{m} / z$ calc'd for $\mathrm{C}_{17} \mathrm{H}_{15} \mathrm{O}_{2} \mathrm{NBrClNa}[\mathrm{M}+\mathrm{Na}]^{+}$: 401.9867, found: 401.9869 . 


\section{4-(Cyclohexylmethyl)-4-(4-methylbenzyl)isoxazolidin-5-one (7b)}

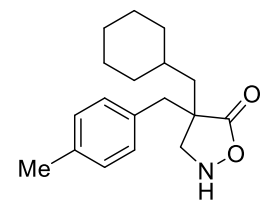

Prepared by the general procedure C from Boc-7b $(600 \mathrm{mg}, 1.54 \mathrm{mmol})$ and isolated by silica gel column chromatgraphy (hexane/ethyl acetate) as a white solid (336 mg, 75\% yield). m.p. $71-73{ }^{\circ} \mathrm{C} ;{ }^{1} \mathbf{H}$ NMR $\left(400 \mathrm{MHz}, \mathrm{CDCl}_{3}\right): \delta 7.15$ - $7.12(\mathrm{~m}, 4 \mathrm{H}), 5.16$ (br, 1H), 3.59-3.50 (m, 2H), $2.99(\mathrm{~d}, J=13.5 \mathrm{~Hz}, 1 \mathrm{H}), 2.75(\mathrm{~d}, J=13.5 \mathrm{~Hz}, 1 \mathrm{H}), 2.33(\mathrm{~s}, 3 \mathrm{H}), 1.71-1.57$ (m, 7H), $1.54-1.45$

$(\mathrm{m}, 1 \mathrm{H}), 1.33-1.12(\mathrm{~m}, 3 \mathrm{H}), 1.10-0.92(\mathrm{~m}, 2 \mathrm{H}) ;{ }^{13} \mathbf{C}$ NMR $\left(101 \mathrm{MHz}, \mathrm{CDCl}_{3}\right): \delta 182.0,137.6,132.7,129.9$, 129.1, 55.9, 49.0, 43.9, 42.1, 35.1, 34.1, 33.4, 26.3, 26.2, 26.0, 21.1; IR (thin film): 3255, 2920, 2849, 1916, 1762 , $1513,1447,1375,1242,1167,1109,1011,865,806,722 \mathrm{~cm}^{-1}$; HRMS (ESI) $m / z$ calc'd for $\mathrm{C}_{18} \mathrm{H}_{26} \mathrm{O}_{2} \mathrm{~N}[\mathrm{M}+\mathrm{H}]^{+}$: 288.1958, found: 288.1958 .

\section{4-(Cyclohexylmethyl)-4-(2-methylbenzyl)isoxazolidin-5-one (7c)}

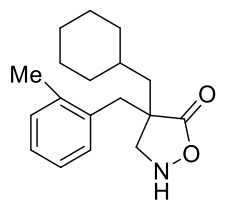

Prepared by the general procedure C from Boc-7c $(680 \mathrm{mg}, 1.75 \mathrm{mmol})$ and isolated by silica gel column chromatgraphy (hexane/ethyl acetate) as a white solid (470 mg, 93\% yield); m.p. 62$64^{\circ} \mathrm{C} ;{ }^{1} \mathbf{H}$ NMR $\left(400 \mathrm{MHz}, \mathrm{CDCl}_{3}\right): \delta 7.22-7.15(\mathrm{~m}, 4 \mathrm{H}), 3.57(\mathrm{~d}, J=11.9 \mathrm{~Hz}, 1 \mathrm{H}), 3.48(\mathrm{~d}, J$ $=11.9 \mathrm{~Hz}, 1 \mathrm{H}), 3.05(\mathrm{~d}, J=14.0 \mathrm{~Hz}, 1 \mathrm{H}), 2.97(\mathrm{~d}, J=14.0 \mathrm{~Hz}, 1 \mathrm{H}), 2.35(\mathrm{~s}, 3 \mathrm{H}), 1.78-1.60(\mathrm{~m}$,

7H), $1.55-1.49(\mathrm{~m}, 7 \mathrm{H}), 1.30-0.98(\mathrm{~m}, 5 \mathrm{H}) ;{ }^{13} \mathbf{C}$ NMR (101 MHz, $\left.\mathrm{CDCl}_{3}\right): \delta$ 182.09, 136.49, 134.28, 131.27, $129.89,127.77,126.73,55.59$, 48.95, 43.94, 37.91, 35.21, 34.31, 33.68, 26.30, 26.19, 26.02, 19.99; IR (thin film): $3255,2920,2849$, 1916, 1762, 1513, 1447, 1375, 1242, 1167, 1136, 1011, 880, 865, 806, $721 \mathrm{~cm}^{-1}$; HRMS (ESI) $m / z$ calc'd for $\mathrm{C}_{18} \mathrm{H}_{26} \mathrm{O}_{2} \mathrm{~N}[\mathrm{M}+\mathrm{H}]^{+}: 288.1958$, found: 288.1959 .

\section{4-([1,1'-Biphenyl]-4-ylmethyl)-4-(cyclohexylmethyl)isoxazolidin-5-one (7d)}

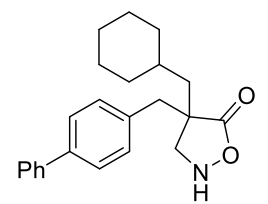

Prepared by the general procedure C from Boc-7d $(520 \mathrm{mg}, 1.15 \mathrm{mmol})$ and isolated by silica gel column chromatgraphy (hexane/ethyl acetate) as a white solid (282 $\mathrm{mg}, 70 \%$ yield). m.p. $103-105{ }^{\circ} \mathrm{C} ;{ }^{1} \mathbf{H}$ NMR $\left(400 \mathrm{MHz}, \mathrm{CDCl}_{3}\right): \delta 7.59-7.56(\mathrm{~m}, 4 \mathrm{H}), 7.46-7.42(\mathrm{~m}, 2 \mathrm{H}), 7.37$ $7.30(\mathrm{~m}, 3 \mathrm{H}), 5.35(\mathrm{br}, 1 \mathrm{H}), 3.59(\mathrm{br}, 2 \mathrm{H}), 3.08(\mathrm{~d}, J=13.4 \mathrm{~Hz}, 1 \mathrm{H}), 2.84(\mathrm{~d}, J=13.5 \mathrm{~Hz}, 1 \mathrm{H})$, $1.74-1.61(\mathrm{~m}, 7 \mathrm{H}), 1.58-1.48(\mathrm{~m}, 1 \mathrm{H}), 1.35-0.95(\mathrm{~m}, 5 \mathrm{H}) ;{ }^{13} \mathbf{C} \mathbf{N M R}\left(101 \mathrm{MHz}, \mathrm{CDCl}_{3}\right): \delta 181.7,140.7,140.2$, $134.8,129.8,128.9,127.8,127.6,127.0,55.9,49.0,43.7,42.0,35.1,34.2,33.5,26.3,26.2$, 26.1; IR (thin film): 3406, 2921, 2848, 1728, 1615, 1568, 1487, 1445, 1206, 1022, $758 \mathrm{~cm}^{-1}$; HRMS (ESI) $\mathrm{m} / z$ calc'd for $\mathrm{C}_{23} \mathrm{H}_{28} \mathrm{O}_{2} \mathrm{~N}$ $[\mathrm{M}+\mathrm{H}]^{+}: 350.2115$, found: 350.2116 .

\section{4-Benzyl-4-((tetrahydro-2H-pyran-4-yl)methyl)isoxazolidin-5-one (7e)}

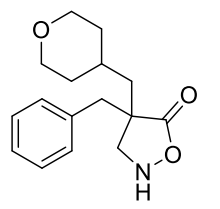

Prepared by the general procedure C from Boc-7e $(667 \mathrm{mg}, 1.77 \mathrm{mmol})$ and isolated by silica gel column chromatgraphy (hexane/ethyl acetate) as a white solid (300 mg, 61\% yield). m.p. 103$105{ }^{\circ} \mathrm{C} ;{ }^{1} \mathbf{H}$ NMR $\left(400 \mathrm{MHz}, \mathrm{CDCl}_{3}\right): \delta 7.38-7.30(\mathrm{~m}, 3 \mathrm{H}), 7.25-7.21(\mathrm{~m}, 2 \mathrm{H}), 3.94(\mathrm{dd}, J=$ 
11.7, 4.4 Hz, 2H), 3.62-3.54 (m, $J=11.8 \mathrm{~Hz}, 2 \mathrm{H}), 3.40$ (ddd, $J=25.0,11.9,2.1 \mathrm{~Hz}, 2 \mathrm{H}), 3.08(\mathrm{~d}, J=13.4 \mathrm{~Hz}, 1 \mathrm{H})$, $2.79(\mathrm{~d}, J=13.4 \mathrm{~Hz}, 1 \mathrm{H}), 1.82-1.68(\mathrm{~m}, 3 \mathrm{H}), 1.63-1.58(\mathrm{~m}, 2 \mathrm{H}), 1.46-1.32(\mathrm{~m}, 2 \mathrm{H}) ;{ }^{13} \mathbf{C} \mathbf{N M R}(101 \mathrm{MHz}$, $\left.\mathrm{CDCl}_{3}\right): \delta 181.3,135.5,129.3,128.0,67.8,67.7,55.8,48.8,43.1,42.4,34.5,33.4,31.5$; IR (thin film): 3390, 2922 , 2838, 1725, 1606, 1499, 1437, 1370, 1282, 1207, 1135, 981, 845, $745 \mathrm{~cm}^{-1}$; HRMS (ESI) $\mathrm{m} / z$ calc'd for $\mathrm{C}_{16} \mathrm{H}_{22} \mathrm{O}_{3} \mathrm{~N}[\mathrm{M}+\mathrm{H}]^{+}: 276.1594$, found: 276.1596 .

\section{4-Benzyl-4-(4-nitrobenzyl)isoxazolidin-5-one (7g)}

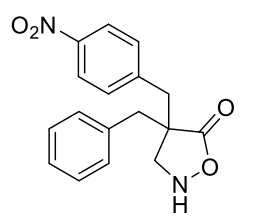

Prepared by the general procedure $\mathrm{C}$ from Boc-7g $(400 \mathrm{mg}, 0.97 \mathrm{mmol})$ and isolated by silica gel column chromatgraphy (hexane/ethyl acetate) as a white solid (280 mg, 92\% yield). m.p. 154-156 ${ }^{\circ} \mathrm{C} ;{ }^{1} \mathbf{H}$ NMR $\left(400 \mathrm{MHz}, \mathrm{CDCl}_{3}\right):{ }^{1} \mathrm{H}$ NMR (400 MHz, $\left.\mathrm{CDCl}_{3}\right) \delta 8.21-8.18(\mathrm{~m}, 2 \mathrm{H})$, $7.41-7.33$ (m, 5H), $7.24-7.22$ (m, 2H), 3.54 (br, 2H), 3.30 (d, $J=13.7 \mathrm{~Hz}, 1 \mathrm{H}), 3.22$ (d, $J=$

$13.5 \mathrm{~Hz}, 1 \mathrm{H}), 3.00(\mathrm{~d}, J=13.7 \mathrm{~Hz}, 1 \mathrm{H}), 2.82(\mathrm{~d}, J=13.5 \mathrm{~Hz}, 1 \mathrm{H}) ;{ }^{13} \mathbf{C} \mathbf{N M R}\left(101 \mathrm{MHz}, \mathrm{CDCl}_{3}\right): \delta 180.1,147.5$, 143.2, 134.9, 131.0, 129.3, 128.2, 124.0, 54.3, 50.6, 42.5, 41.7; IR (thin film): 3379, 2954, 2850, 1718, 1602, 1514, 1344, 1200, 1106, 970, 856, $746 \mathrm{~cm}^{-1}$; HRMS (ESI) $\mathrm{m} / z$ calc'd for $\mathrm{C}_{17} \mathrm{H}_{16} \mathrm{O}_{4} \mathrm{~N}_{2} \mathrm{Na}[\mathrm{M}+\mathrm{Na}]^{+}: 335.1002$, found: 335.1002 .

\section{Copper-catalyzed $\beta$-amino acid synthesis}

\section{4-1. Catalytic asymmetric desymmetrization}

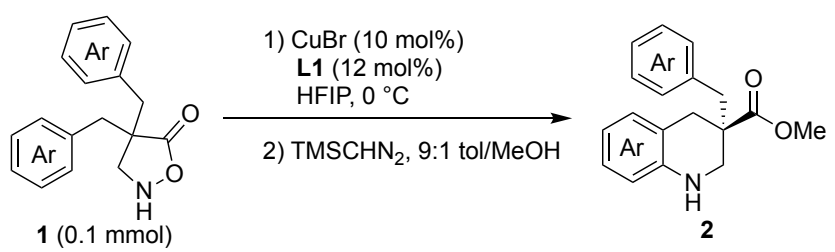

General procedure D: To a flame-dried $10 \mathrm{~mL}$ test tube equipped with a magnetic stirring bar were added L1 (2.9 $\mathrm{mg}, 0.012 \mathrm{mmol}, 12 \mathrm{~mol} \%)$ and $\mathrm{CuBr}(1.4 \mathrm{mg}, 0.01 \mathrm{mmol}, 10 \mathrm{~mol} \%)$ in glove box, followed by the addition of HFIP $(0.5 \mathrm{~mL})$ and the mixture was stirred for 15 minutes at an ambient temperature. After that, the reaction was cooled with the ice bath, and substrate $1(0.1 \mathrm{mmol})$ with the $0.5 \mathrm{~mL}$ HFIP was added as a solution. The reaction mixture was stirred for a specific time at the same temperature and was quenched by the addition of aq EDTA and water. The aqueous layer was extracted with EtOAc (3x). The combined organic layers were concentrated under reduced pressure. The obtained residue was dissolved in 9:1 toluene: $\mathrm{MeOH}(1.0 \mathrm{~mL}, 0.1 \mathrm{M})$. To the resulting solution cooled with an ice bath was added (trimethylsilyl)diazomethane (2.0 $\mathrm{M}$ in $\mathrm{Et}_{2} \mathrm{O}, 75 \mu \mathrm{L}, 1.5$ equiv). After $15 \mathrm{~min}$, the reaction was quenched by the addition of aq $\mathrm{AcOH}$, followed by sat aq $\mathrm{NaHCO}_{3}$. The aqueous layer was extracted with EtOAc (3x). The combined organic layers were concentrated under reduced pressure to give the crude product, which was purified by silica gel column chromatography to afford pure product 2 . 


\section{Methyl (R)-3-benzyl-1,2,3,4-tetrahydroquinoline-3-carboxylate (2a)}

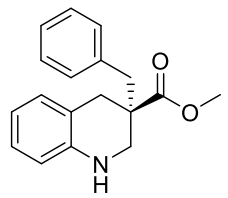

Prepared by the general procedure D from 1a $(26.7 \mathrm{mg}, 0.1 \mathrm{mmol})$, and isolated by silica gel column chromatgraphy (hexane/ethyl acetate) as a white solid (26.0 mg, 93\% yield). m.p. 67$69{ }^{\circ} \mathrm{C} ;{ }^{1} \mathbf{H}$ NMR $\left(400 \mathrm{MHz}, \mathrm{CDCl}_{3}\right): \delta^{1} \mathrm{H} \mathrm{NMR}\left(400 \mathrm{MHz}, \mathrm{CDCl}_{3}\right) \delta 7.28-7.26(\mathrm{~m}, 1 \mathrm{H}), 7.25-$ $7.22(\mathrm{~m}, 2 \mathrm{H}), 7.10-7.08(\mathrm{~m}, 2 \mathrm{H}), 7.01-6.97(\mathrm{~m}, 2 \mathrm{H}), 6.65(\mathrm{td}, J=7.4,1.1 \mathrm{~Hz}, 1 \mathrm{H}), 6.52(\mathrm{~d}, J=$ $7.9 \mathrm{~Hz}, 1 \mathrm{H}), 3.89$ (br, 1H), 3.63 (s, 3H), 3.48 (dd, $J=11.6,1.1 \mathrm{~Hz}, 1 \mathrm{H}), 3.22$ (dd, $J=11.6,1.6 \mathrm{~Hz}, 1 \mathrm{H}), 3.09$ (d, $J$ $=16.2 \mathrm{~Hz}, 1 \mathrm{H}), 2.96(\mathrm{dd}, J=42.8,13.4 \mathrm{~Hz}, 2 \mathrm{H}), 2.82(\mathrm{~d}, J=16.2 \mathrm{~Hz}, 1 \mathrm{H}) ;{ }^{13} \mathbf{C} \mathbf{N M R}\left(101 \mathrm{MHz}, \mathrm{CDCl}_{3}\right): \delta 175.2$, 143.2, 137.0, 129.9, 129.8, 128.2, 127.0, 126.7, 119.0, 117.6, 114.1, 51.8, 46.9, 45.7, 41.3, 34.9; HRMS (ESI) $\mathrm{m} / \mathrm{z}$ calc'd for $\mathrm{C}_{18} \mathrm{H}_{20} \mathrm{O}_{2} \mathrm{~N}[\mathrm{M}+\mathrm{H}]^{+}:$282.1489, found: $282.1489 ;[\alpha]_{\mathrm{D}}^{27}+107.52$ (c 1.2, $\mathrm{CHCl}_{3}, 94 \%$ ee sample). Enantiomeric excess of the product was determined to be $94 \%$ ee by chiral stationary phase HPLC analysis (CHIRALPAK IA $(\phi 0.46 \mathrm{~cm} \times 25 \mathrm{~cm}), n$-hexane $/ 2$-propanol $=80 / 20$, flow rate $1.0 \mathrm{~mL} / \mathrm{min}$, detection at $254 \mathrm{~nm}$, $\mathrm{t}_{R}=6.30 \mathrm{~min}$ (major), $11.10 \mathrm{~min}$ (minor).
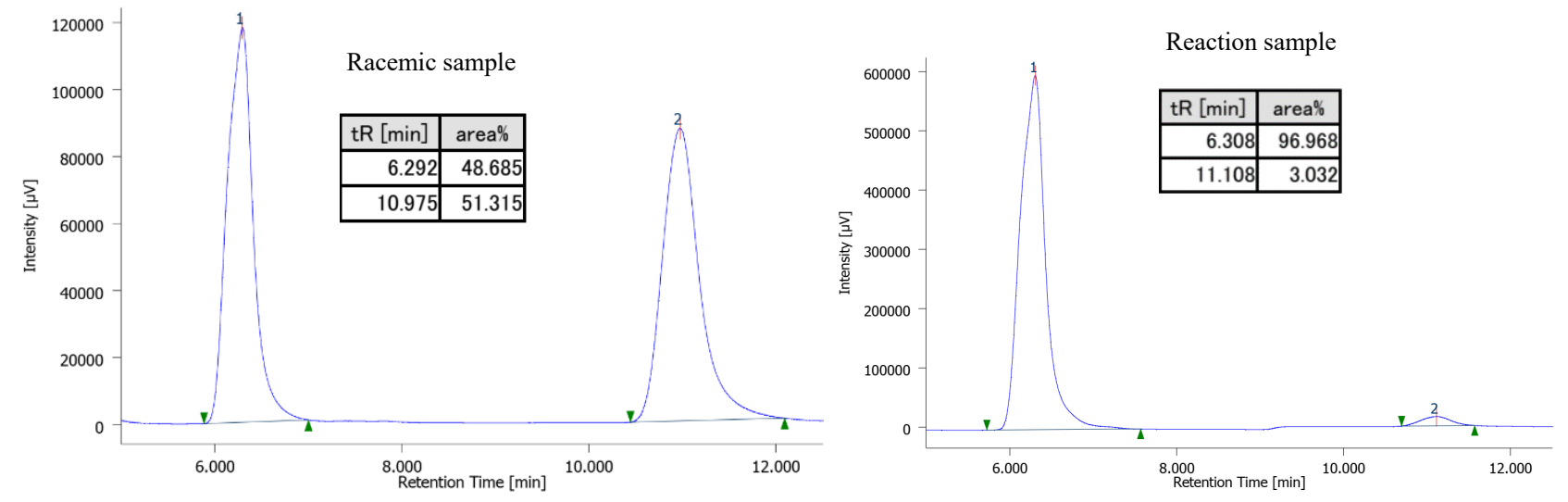

\section{Methyl (R)-7-methyl-3-(4-methylbenzyl)-1,2,3,4-tetrahydroquinoline-3-carboxylate (2b)}

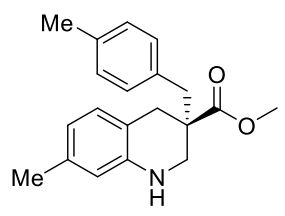

Prepared by the general procedure D from $1 \mathbf{b}(29.5 \mathrm{mg}, 0.1 \mathrm{mmol})$, and isolated by silica gel column chromatgraphy (hexane/ethyl acetate) as a white solid (28.1 mg, 91\% yield). m.p. 76$78{ }^{\circ} \mathrm{C} ;{ }^{1} \mathbf{H}$ NMR $\left(400 \mathrm{MHz}, \mathrm{CDCl}_{3}\right): \delta 7.07$ (d, $\left.J=7.8 \mathrm{~Hz}, 2 \mathrm{H}\right), 6.98(\mathrm{~d}, J=8.0 \mathrm{~Hz}, 2 \mathrm{H}), 6.88$ $(\mathrm{d}, J=7.6 \mathrm{~Hz}, 1 \mathrm{H}), 6.49(\mathrm{dd}, J=7.6,1.0 \mathrm{~Hz}, 1 \mathrm{H}), 6.36(\mathrm{~s}, 1 \mathrm{H}), 3.82(\mathrm{~s}, 1 \mathrm{H}), 3.63(\mathrm{~s}, 3 \mathrm{H}), 3.45$ (dd, $J=11.6,1.0 \mathrm{~Hz}, 1 \mathrm{H}), 3.19(\mathrm{dd}, J=11.6,1.4 \mathrm{~Hz}, 1 \mathrm{H}), 3.04$ (d, $J=16.1 \mathrm{~Hz}, 1 \mathrm{H}), 2.91$ (dd, $J=43.3,13.4 \mathrm{~Hz}$, 2H), 2.77 (d, $J=16.1 \mathrm{~Hz}, 1 \mathrm{H}), 2.32(\mathrm{~s}, 3 \mathrm{H}), 2.24(\mathrm{~s}, 3 \mathrm{H}) ;{ }^{13} \mathbf{C} \mathbf{N M R}\left(101 \mathrm{MHz}, \mathrm{CDCl}_{3}\right): \delta$ 175.3, 143.1, 136.7, 136.2, 133.9, 129.8, 129.7, 128.9, 118.7, 116.2, 114.6, 51.7, 46.9, 45.9, 40.9, 34.5, 21.2, 21.1; IR (thin film): 3405, 3006, 2920, 2857, 1700, 1618, 1513, 1440, 1285, 1180, 1102, $803 \mathrm{~cm}^{-1}$; HRMS (ESI) $\mathrm{m} / z$ calc'd for $\mathrm{C}_{20} \mathrm{H}_{24} \mathrm{O}_{2} \mathrm{~N}$ $[\mathrm{M}+\mathrm{H}]^{+}: 310.1802$, found: $310.1804 ;[\alpha]_{\mathrm{D}}{ }^{27}+40.23\left(c 1.0, \mathrm{CHCl}_{3}, 90 \%\right.$ ee sample). Enantiomeric excess of the product was determined to be $90 \%$ ee by chiral stationary phase HPLC analysis (CHIRALPAK IA $(\phi 0.46 \mathrm{~cm} \times 25$ $\mathrm{cm}$ ), $n$-hexane/2-propanol $=80 / 20$, flow rate $1.0 \mathrm{~mL} / \mathrm{min}$, detection at $254 \mathrm{~nm}, \mathrm{t}_{R}=6.17$ min (major), $9.20 \mathrm{~min}$ (minor). 

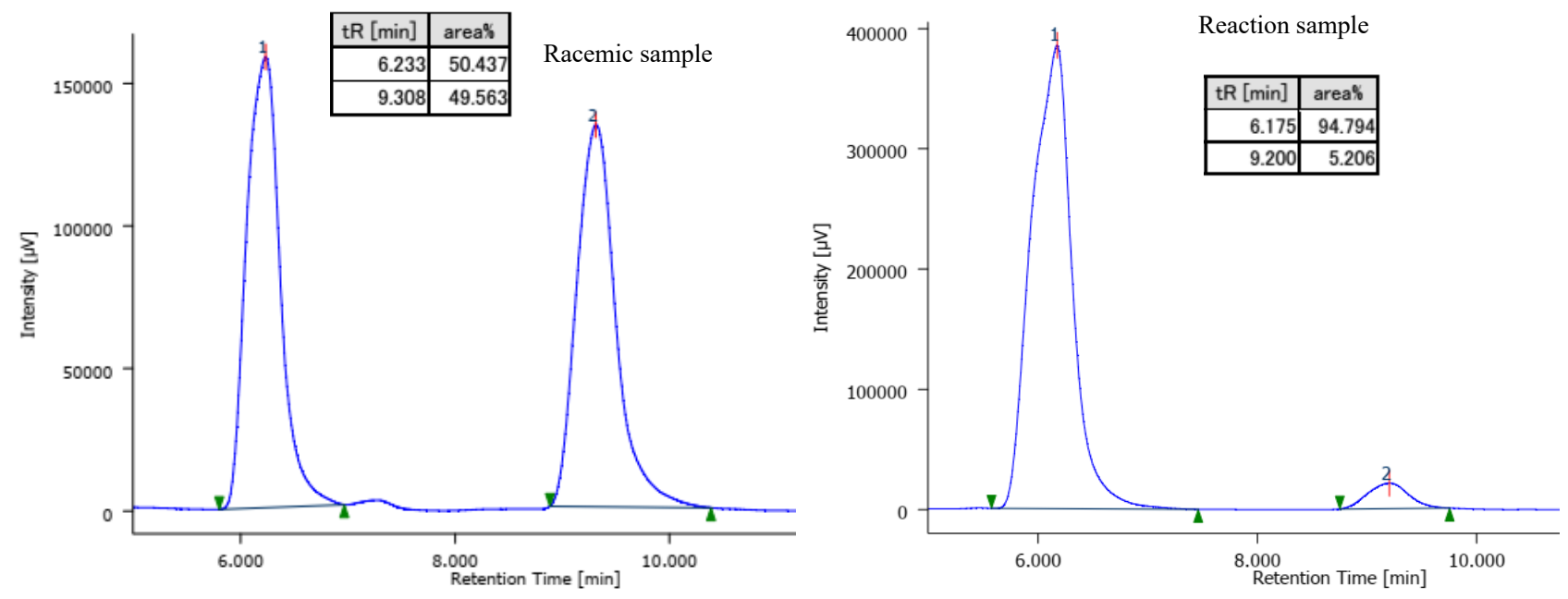

\section{Methyl (R)-5-methyl-3-(2-methylbenzyl)-1,2,3,4-tetrahydroquinoline-3-carboxylate (2c)}

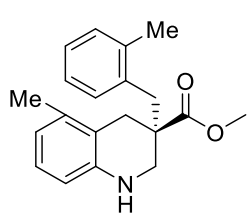

Prepared by the general procedure D from 1c $(29.5 \mathrm{mg}, 0.1 \mathrm{mmol})$, and isolated by silica gel column chromatgraphy (hexane/ethyl acetate) as a colorless liquid (25.9 $\mathrm{mg}, 84 \%$ yield). ${ }^{1} \mathbf{H}$ NMR (400 MHz, $\left.\mathrm{CDCl}_{3}\right): \delta 7.14-7.07(\mathrm{~m}, 3 \mathrm{H}), 7.03-7.01(\mathrm{~m}, 1 \mathrm{H}), 6.90(\mathrm{t}, J=7.7 \mathrm{~Hz}, 1 \mathrm{H})$, $6.55(\mathrm{~d}, J=7.4 \mathrm{~Hz}, 1 \mathrm{H}), 6.40(\mathrm{~d}, J=8.0 \mathrm{~Hz}, 1 \mathrm{H}), 3.85$ (br, $1 \mathrm{H}), 3.63$ (s, 3H), 3.49 (dd, $J=11.4$, $1.3 \mathrm{~Hz}, 1 \mathrm{H}), 3.26(\mathrm{dd}, J=11.4,1.3 \mathrm{~Hz}, 1 \mathrm{H}), 3.06(\mathrm{~s}, 2 \mathrm{H}), 3.01(\mathrm{~d}, J=16.8 \mathrm{~Hz}, 1 \mathrm{H}), 2.68(\mathrm{~d}, J=16.7 \mathrm{~Hz}, 1 \mathrm{H}), 2.22$ (s, 3H), 2.17 (s, 3H); ${ }^{13} \mathbf{C}$ NMR (101 MHz, $\left.\mathrm{CDCl}_{3}\right): \delta 175.7,143.2,137.5,137.1,135.4,130.5,126.7,126.4,125.6$, 119.5, 118.1, 112.2, 51.9, 47.2, 46.2, 38.0, 31.9, 19.6, 19.4; IR (thin film): 3405, 3017, 2945, 2838, 1723, 1590, 1482, 1433, 1359, 1282, 1198, 1115, 768, $744 \mathrm{~cm}^{-1}$; HRMS (ESI) $m / z$ calc'd for $\mathrm{C}_{20} \mathrm{H}_{24} \mathrm{O}_{2} \mathrm{~N}[\mathrm{M}+\mathrm{H}]^{+}: 310.1802$, found: $310.1804 ;[\alpha]_{\mathrm{D}}{ }^{27}+48.63\left(c 1.0, \mathrm{CHCl}_{3}, 85 \%\right.$ ee sample). Enantiomeric excess of the product was determined to be $85 \%$ ee by chiral stationary phase HPLC analysis (CHIRALPAK IA $(\phi 0.46 \mathrm{~cm} \times 25 \mathrm{~cm}), n$-hexane/2propanol $=80 / 20$, flow rate $1.0 \mathrm{~mL} / \mathrm{min}$, detection at $254 \mathrm{~nm}, \mathrm{t}_{R}=7.24 \mathrm{~min}$ (major), $11.34 \mathrm{~min}$ (minor).
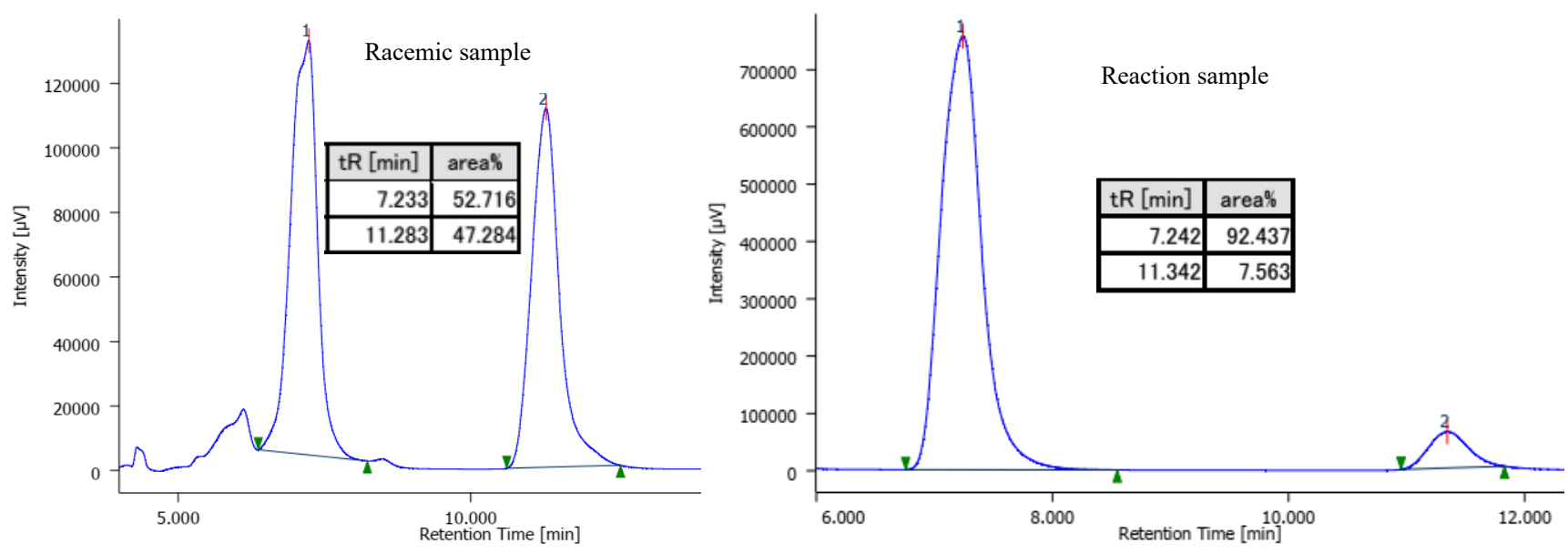


\section{Methyl (R)-3-([1,1'-biphenyl]-4-ylmethyl)-7-phenyl-1,2,3,4-tetrahydroquinoline-3-carboxylate (2d)}

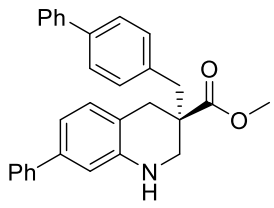

Prepared by the general procedure D from $1 \mathrm{~d}(41.9 \mathrm{mg}, 0.1 \mathrm{mmol})$, and isolated by silica gel column chromatgraphy (hexane/ethyl acetate) as a white solid (38.1 $\mathrm{mg}, 88 \%$ yield). m.p. 184-186 ${ }^{\circ} \mathrm{C} ;{ }^{1} \mathbf{H}$ NMR $\left(400 \mathrm{MHz}, \mathrm{CDCl}_{3}\right): \delta 7.60-7.50(\mathrm{~m}, 6 \mathrm{H}), 7.45-7.38(\mathrm{~m}, 4 \mathrm{H}), 7.36-$ $7.29(\mathrm{~m}, 2 \mathrm{H}), 7.19-7.1(\mathrm{~m}, 2 \mathrm{H}), 7.08(\mathrm{~d}, J=7.8 \mathrm{~Hz}, 1 \mathrm{H}), 6.92(\mathrm{dd}, J=7.8,1.8 \mathrm{~Hz}, 1 \mathrm{H}), 6.78$ $(\mathrm{d}, J=1.7 \mathrm{~Hz}, 1 \mathrm{H}), 4.07$ (br, 1H), 3.68 (s, 3H), 3.56 (dd, $J=11.6,0.9 \mathrm{~Hz}, 1 \mathrm{H}), 3.31(\mathrm{dd}, J=11.6,1.3 \mathrm{~Hz}, 1 \mathrm{H})$, $3.17(\mathrm{~d}, J=16.3 \mathrm{~Hz}, 1 \mathrm{H}), 3.04(\mathrm{dd}, J=40.8,13.4 \mathrm{~Hz}, 2 \mathrm{H}), 2.90(\mathrm{~d}, J=16.2 \mathrm{~Hz}, 1 \mathrm{H}) ;{ }^{13} \mathbf{C ~ N M R}\left(101 \mathrm{MHz}, \mathrm{CDCl}_{3}\right)$ : $\delta 175.2,143.5,141.4,140.9,140.3,139.7,136.0,130.3,130.3,128.8,128.6,127.2,127.0,127.0,127.0,126.9$, 118.4, 116.8, 112.8, 51.9, 47.1, 45.9, 41.0, 34.7; IR (thin film): 3424, 2949, 2918, 2848, 1725, 1654, 1581, 1486, 1406, 1113, 1024, 820, $756 \mathrm{~cm}^{-1}$; HRMS (ESI) $\mathrm{m} / z$ calc'd for $\mathrm{C}_{30} \mathrm{H}_{28} \mathrm{O}_{2} \mathrm{~N}$ [M $\left.+\mathrm{H}\right]^{+}:$434.2115, found: 434.2114; $[\alpha]_{\mathrm{D}}^{27}+37.85$ ( $c 1.0, \mathrm{CHCl}_{3}, 92 \%$ ee sample). Enantiomeric excess of the product was determined to be $92 \%$ ee by chiral stationary phase HPLC analysis (CHIRALPAK IA $(\phi 0.46 \mathrm{~cm} \times 25 \mathrm{~cm}), n$-hexane/2-propanol $=90 / 10$, flow rate $1.0 \mathrm{~mL} / \mathrm{min}$, detection at $254 \mathrm{~nm}, \mathrm{t}_{R}=31.30 \mathrm{~min}$ (major), $39.55 \mathrm{~min}$ (minor).
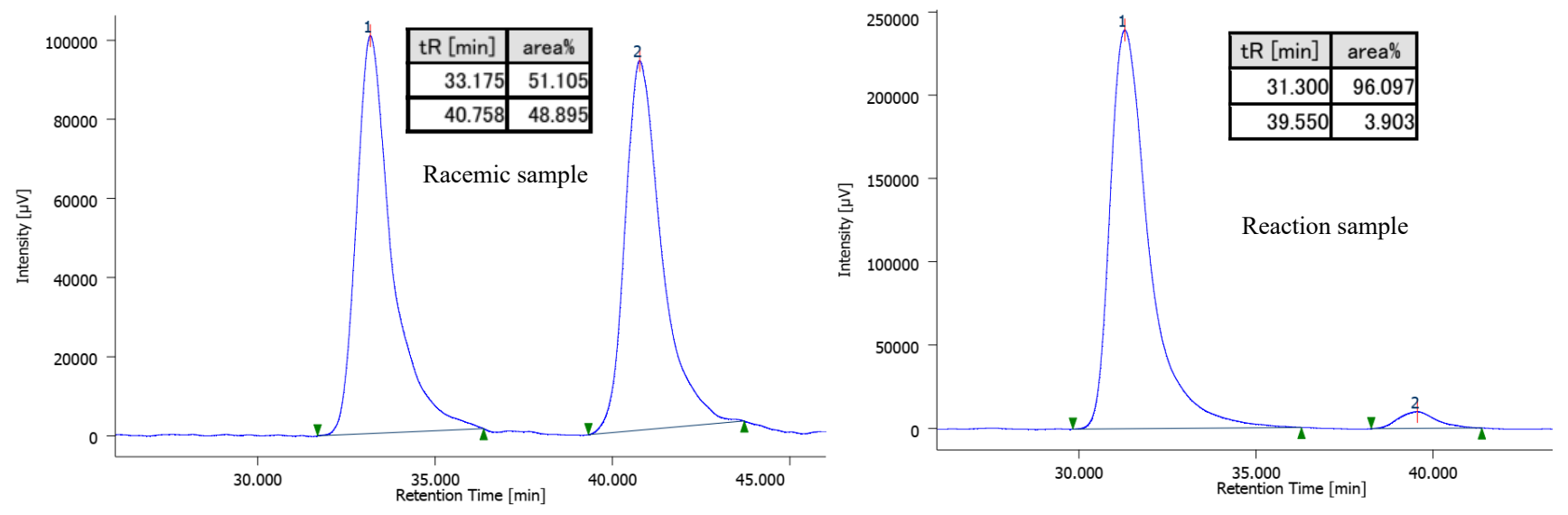

\section{Methyl (R)-7-(tert-butyl)-3-(4-(tert-butyl)benzyl)-1,2,3,4-tetrahydroquinoline-3-carboxylate (2e)}

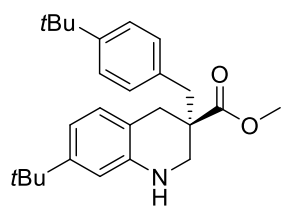

Prepared by the general procedure D from 1 e $(37.9 \mathrm{mg}, 0.1 \mathrm{mmol})$, and isolated by silica gel column chromatgraphy (hexane/ethyl acetate) as a white solid (35.3 $\mathrm{mg}, 90 \%$ yield). m.p. 127-129 ${ }^{\circ} \mathrm{C} ;{ }^{1} \mathbf{H}$ NMR (400 MHz, $\left.\mathrm{CDCl}_{3}\right): \delta 7.29$ - $7.26(\mathrm{~m}, 2 \mathrm{H}), 7.04-7.01(\mathrm{~m}, 2 \mathrm{H}), 6.94(\mathrm{~d}$, $J=7.9 \mathrm{~Hz}, 1 \mathrm{H}), 6.71(\mathrm{dd}, J=7.9,1.9 \mathrm{~Hz}, 1 \mathrm{H}), 6.55(\mathrm{~d}, J=1.9 \mathrm{~Hz}, 1 \mathrm{H}), 3.83(\mathrm{br}, 1 \mathrm{H}), 3.65$ (s, 3H), $3.46(\mathrm{dd}, J=11.5,0.9 \mathrm{~Hz}, 1 \mathrm{H}), 3.19(\mathrm{dd}, J=11.6,1.3 \mathrm{~Hz}, 1 \mathrm{H}), 3.07$ (d, $J=16.2 \mathrm{~Hz}, 1 \mathrm{H}), 2.93(\mathrm{dd}, J=$ 37.3, $13.4 \mathrm{~Hz}, 2 \mathrm{H}), 2.80(\mathrm{~d}, J=16.2 \mathrm{~Hz}, 1 \mathrm{H}), 1.31(\mathrm{~s}, 9 \mathrm{H}), 1.28(\mathrm{~s}, 9 \mathrm{H}) ;{ }^{13} \mathbf{C}$ NMR $\left(101 \mathrm{MHz}, \mathrm{CDCl}_{3}\right): \delta 175.4$, $150.1,149.4,142.8,133.9,129.5,129.5,125.0,116.4,115.2,111.3,51.7,46.9,45.9,40.9,34.4,34.3,31.4$; IR (thin film): 3407, 2961, 2867, 1730, 1617, 1579, 1476, 1363, 1285, 1201, 1021, 841, $801 \mathrm{~cm}^{-1}$; HRMS (ESI) m/z calc'd for $\mathrm{C}_{26} \mathrm{H}_{36} \mathrm{O}_{2} \mathrm{~N}[\mathrm{M}+\mathrm{H}]^{+}: 394.2741$, found: $394.2740 ;[\alpha]_{\mathrm{D}}{ }^{27}+39.19$ (c 1.0, $\mathrm{CHCl}_{3}, 89 \%$ ee sample). Enantiomeric excess of the product was determined to be $89 \%$ ee by chiral stationary phase HPLC analysis 
(CHIRALPAK OJH ( $\phi 0.46 \mathrm{~cm} \times 25 \mathrm{~cm}), n$-hexane $/ 2$-propanol $=95 / 5$, flow rate $0.5 \mathrm{~mL} / \mathrm{min}$, detection at $254 \mathrm{~nm}$, $\mathrm{t}_{R}=9.60 \mathrm{~min}$ (minor), $10.91 \mathrm{~min}$ (major).
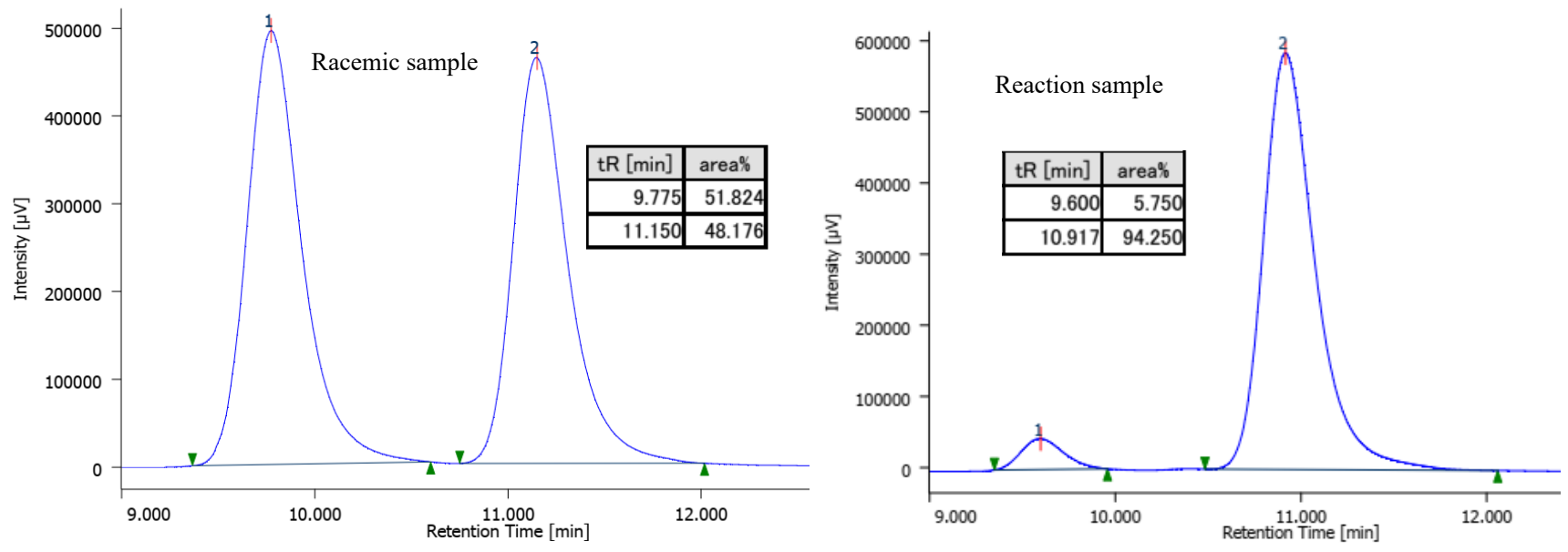

\section{Methyl (R)-7-bromo-3-(4-bromobenzyl)-1,2,3,4-tetrahydroquinoline-3-carboxylate (2f)}

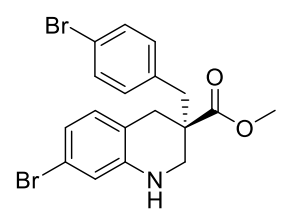

Prepared by the general procedure D from 1 f $(42.2 \mathrm{mg}, 0.1 \mathrm{mmol})$, and isolated by silica gel column chromatgraphy (hexane/ethyl acetate) as a white solid (19.6 mg, 45\% yield). m.p. 144 $146{ }^{\circ} \mathrm{C} ;{ }^{1} \mathbf{H}$ NMR $\left(400 \mathrm{MHz}, \mathrm{CDCl}_{3}\right): \delta 7.40-7.37(\mathrm{~m}, 2 \mathrm{H}), 6.95-6.92(\mathrm{~m}, 2 \mathrm{H}), 6.82(\mathrm{~d}, J=$ $8.0 \mathrm{~Hz}, 1 \mathrm{H}), 6.75(\mathrm{dd}, J=8.0,1.9 \mathrm{~Hz}, 1 \mathrm{H}), 6.67(\mathrm{~d}, J=1.8 \mathrm{~Hz}, 1 \mathrm{H}), 3.97(\mathrm{br}, 1 \mathrm{H}), 3.63(\mathrm{~s}, 3 \mathrm{H})$, $3.45(\mathrm{~d}, J=11.7 \mathrm{~Hz}, 1 \mathrm{H}), 3.18(\mathrm{~d}, J=11.7 \mathrm{~Hz}, 1 \mathrm{H}), 2.99$ (d, $J=16.2 \mathrm{~Hz}, 1 \mathrm{H}), 2.88$ (dd, $J=39.3,13.5 \mathrm{~Hz}, 2 \mathrm{H})$, $2.70(\mathrm{~d}, J=16.1 \mathrm{~Hz}, 1 \mathrm{H}) ;{ }^{13} \mathbf{C}$ NMR $\left(101 \mathrm{MHz}, \mathrm{CDCl}_{3}\right): \delta 174.6,144.3,135.7,131.5,131.4,131.2,120.9,120.5$, 120.4, 117.6, 116.4, 52.0, 46.6, 45.3, 40.4, 34.4; IR (thin film): 3405, 2949, 2847, 1730, 1603, 1579, 1490, 1439, 1285, 1197, 1087, 1015, 841, $788 \mathrm{~cm}^{-1}$; HRMS (ESI) $\mathrm{m} / z$ calc'd for $\mathrm{C}_{18} \mathrm{H}_{18} \mathrm{O}_{2} \mathrm{NBr}_{2}[\mathrm{M}+\mathrm{H}]^{+}: 437.9699$, found: 437.9697; $[\alpha]_{\mathrm{D}}{ }^{27}+44.16$ (c 1.0, $\mathrm{CHCl}_{3}, 87 \%$ ee sample). Enantiomeric excess of the product was determined to be $87 \%$ ee by chiral stationary phase HPLC analysis (CHIRALPAK IA $(\phi 0.46 \mathrm{~cm} \times 25 \mathrm{~cm}), n$-hexane/2-propanol $=$ $90 / 10$, flow rate $1.0 \mathrm{~mL} / \mathrm{min}$, detection at $254 \mathrm{~nm}, \mathrm{t}_{R}=12.68 \mathrm{~min}$ (major), $14.13 \mathrm{~min}$ (minor).

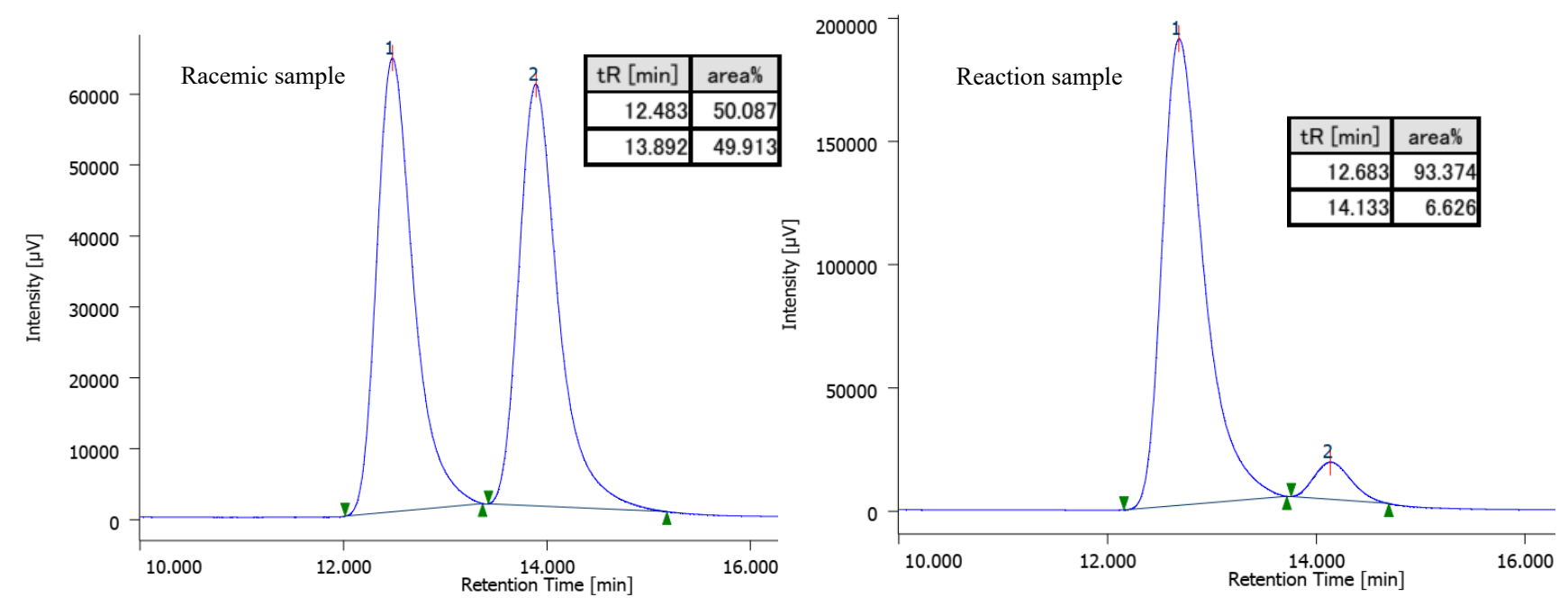




\section{Methyl (R)-5-chloro-3-(2-chlorobenzyl)-1,2,3,4-tetrahydroquinoline-3-carboxylate (2g)}

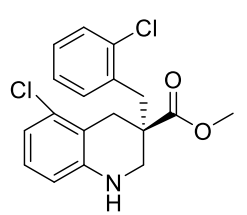

Prepared by the general procedure D from $1 \mathrm{~g}(33.5 \mathrm{mg}, 0.1 \mathrm{mmol})$, and isolated by silica gel column chromatgraphy (hexane/ethyl acetate) as a white solid (15.7 mg, 45\% yield). m.p. 112 $114{ }^{\circ} \mathrm{C} ;{ }^{1} \mathbf{H}$ NMR (400 MHz, $\left.\mathrm{CDCl}_{3}\right): \delta 7.38-7.34(\mathrm{~m}, 1 \mathrm{H}), 7.19-7.15(\mathrm{~m}, 2 \mathrm{H}), 7.14-7.11(\mathrm{~m}$, $1 \mathrm{H}), 6.91(\mathrm{t}, J=8.0 \mathrm{~Hz}, 1 \mathrm{H}), 6.72(\mathrm{dd}, J=7.9,1.0 \mathrm{~Hz}, 1 \mathrm{H}), 6.41(\mathrm{dd}, J=8.0,1.0 \mathrm{~Hz}, 1 \mathrm{H}), 4.01$ (br, 1H), $3.63(\mathrm{~s}, 3 \mathrm{H}), 3.53$ (dd, $J=11.7,1.6 \mathrm{~Hz}, 1 \mathrm{H}), 3.26(\mathrm{dd}, J=11.7,1.2 \mathrm{~Hz}, 1 \mathrm{H}), 3.21-3.12(\mathrm{~m}, 3 \mathrm{H}), 2.90(\mathrm{~d}$, $J=17.1 \mathrm{~Hz}, 1 \mathrm{H}) ;{ }^{13} \mathrm{C}$ NMR $\left(101 \mathrm{MHz}, \mathrm{CDCl}_{3}\right): \delta 174.6,144.7,135.0,135.0,134.6,132.0,129.7,128.3,127.4$, 126.6, 118.2, 117.3, 112.4, 52.2, 47.0, 45.7, 38.4, 32.4; IR (thin film): 3419, 3066, 2950, 2838, 1731, 1667, 1600, 1475, 1362, 1204, 1052, 956, $752 \mathrm{~cm}^{-1}$; HRMS (ESI) $\mathrm{m} / z$ calc'd for $\mathrm{C}_{18} \mathrm{H}_{18} \mathrm{O}_{2} \mathrm{NCl}_{2}[\mathrm{M}+\mathrm{H}]^{+}: 350.0709$, found: $350.0710 ;[\alpha]_{\mathrm{D}}^{27}+37.36\left(c 0.75, \mathrm{CHCl}_{3}, 89 \%\right.$ ee sample). Enantiomeric excess of the product was determined to be $89 \%$ ee by chiral stationary phase HPLC analysis (CHIRALPAK IA $(\phi 0.46 \mathrm{~cm}$ x $25 \mathrm{~cm}), n$-hexane/2-propanol = $80 / 20$, flow rate $1.0 \mathrm{~mL} / \mathrm{min}$, detection at $254 \mathrm{~nm}, \mathrm{t}_{R}=6.77 \mathrm{~min}$ (major), $12.80 \mathrm{~min}$ (minor).
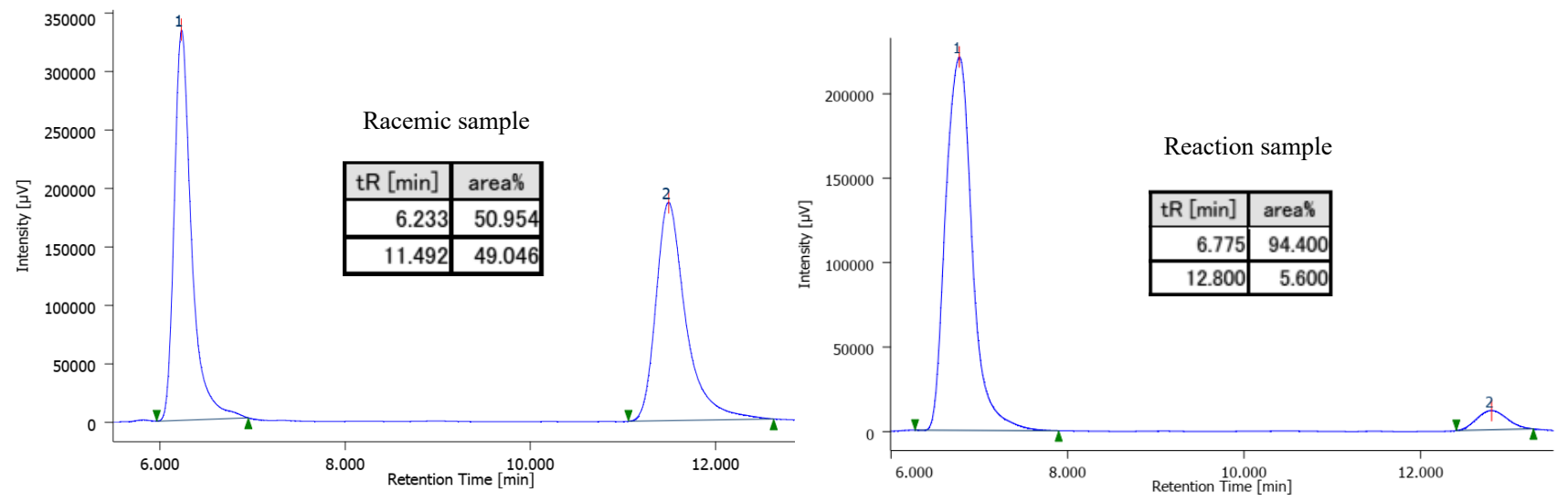

\section{4-2. Parallel kinetic resolution}
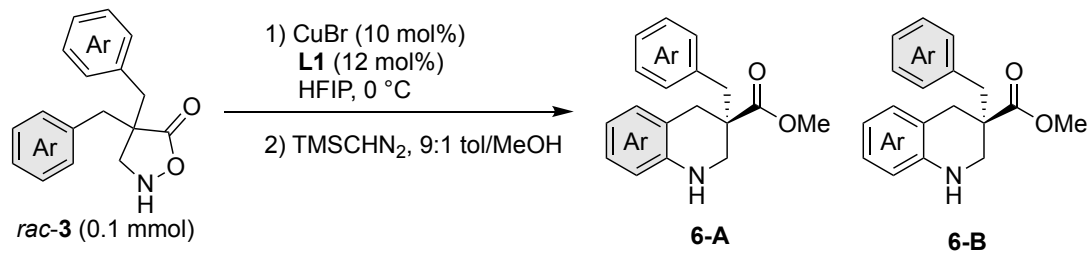

General procedure E: To a flame-dried $10 \mathrm{~mL}$ test tube equipped with a magnetic stirring bar were added L1 (2.9 $\mathrm{mg}, 0.012 \mathrm{mmol}, 12 \mathrm{~mol} \%)$ and $\mathrm{CuBr}(1.4 \mathrm{mg}, 0.01 \mathrm{mmol}, 10 \mathrm{~mol} \%)$ in glove box, followed by the addition of HFIP $(0.50 \mathrm{~mL})$ and the mixture was stirred for $15 \mathrm{~min}$ at an ambient temperature. After that resulting solution cooled to $0{ }^{\circ} \mathrm{C}$ and substrate $\mathrm{rac}-3(0.1 \mathrm{mmol})$ with the $0.50 \mathrm{~mL}$ HFIP was added as a solution. The reaction mixture was stirred for the indicated time at the same temperature and was quenched by the addition of aq EDTA and water. The aqueous layer was extracted with EtOAc (3x). The combined organic layers were concentrated under reduced pressure. The obtained residue was dissolved in 9:1 toluene: $\mathrm{MeOH}(1.0 \mathrm{~mL}, 0.1 \mathrm{M})$. To the solution 
cooled with an ice bath was added (trimethylsilyl)diazomethane (2.0 $\mathrm{M}$ in $\mathrm{Et}_{2} \mathrm{O}, 75 \mu \mathrm{L}, 1.5$ equiv). After 15 min, the reaction was quenched by the addition of aq $\mathrm{AcOH}$, followed by sat aq $\mathrm{NaHCO}_{3}$. The aqueous layer was extracted with EtOAc $(3 \mathrm{x})$. The combined organic layers were concentrated under reduced pressure to give the crude product, which was purified by silica gel column chromatography. After purification, the product ratio and ee were determined by using the chiral stationary phase HPLC column.

Mixture of methyl (R)-3-(4-methylbenzyl)-1,2,3,4-tetrahydroquinoline-3-carboxylate (6a-A) and methyl (R)3-benzyl-7-methyl-1,2,3,4-tetrahydroquinoline-3-carboxylate (6a-B)

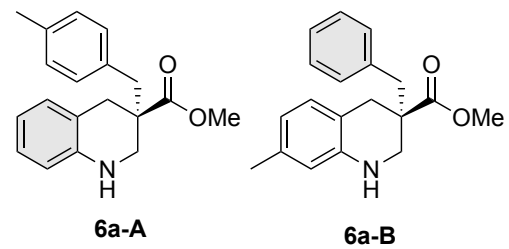

Prepared by the general procedure E from 3a $(28.1 \mathrm{mg}, 0.1 \mathrm{mmol})$, stirred for 2 $\mathrm{h}$, and isolated by silica gel column chromatgraphy (hexane/ethyl acetate) as a 47:53 mixture (26.5 mg, 90\% yield). White solid; m.p. $72-74{ }^{\circ} \mathrm{C} ;{ }^{1} \mathbf{H}$ NMR $\left(400 \mathrm{MHz}, \mathrm{CDCl}_{3}\right): \delta 7.28-7.26(\mathrm{~m}, 1 \mathrm{H}), 7.25-7.19(\mathrm{~m}, 2 \mathrm{H}), 7.10-7.06(\mathrm{~m}$, 4H), $7.02-6.96(\mathrm{~m}, 4 \mathrm{H}), 6.88(\mathrm{~d}, J=7.6 \mathrm{~Hz}, 1 \mathrm{H}), 6.65(\mathrm{td}, J=7.4,1.1 \mathrm{~Hz}, 1 \mathrm{H}), 6.53-6.47(\mathrm{~m}, 2 \mathrm{H}), 6.36(\mathrm{~s}, 1 \mathrm{H})$, $3.86(\mathrm{~d}, J=15.5 \mathrm{~Hz}, 2 \mathrm{H}), 3.63(\mathrm{~s}, 2.81 \mathrm{H}), 3.63(\mathrm{~s}, 3.19 \mathrm{H}), 3.48$ (dd, $J=6.6,1.0 \mathrm{~Hz}, 0.94 \mathrm{H}), 3.45(\mathrm{dd}, J=6.6,1.0$ $\mathrm{Hz}, 1.13 \mathrm{H}), 3.22(\mathrm{dd}, J=4.4,1.4 \mathrm{~Hz}, 1.13 \mathrm{H}), 3.19$ (dd, $J=4.4,1.5 \mathrm{~Hz}, 0.93 \mathrm{H}), 3.10-2.76(\mathrm{~m}, 8 \mathrm{H}), 2.31(\mathrm{~s}, 2.82$ $\mathrm{H}), 2.23$ (s, $3.18 \mathrm{H}) ;{ }^{13} \mathbf{C}$ NMR (101 MHz, $\left.\mathrm{CDCl}_{3}\right): \delta 175.2,175.2,143.3,143.1,137.1,136.7,136.3,133.8,129.9$, $129.8,129.8,129.7,128.9,128.2$, 127.0, 126.7, 119.1, 118.7, 117.6, 116.1, 114.6, 114.1, 51.7, 51.7, 47.0, 46.9, 45.9, 45.7, 41.3 41.0, 34.8, 34.6, 21.2, 21.1; IR (thin film): 3403, 2947, 2954, 1722, 1617, 1584, 1491, 1433, 1360, 1285, 1195, 1111, 1032, 864, 740, $645 \mathrm{~cm}^{-1}$; HRMS (ESI) $\mathrm{m} / z$ calc'd for $\mathrm{C}_{19} \mathrm{H}_{22} \mathrm{O}_{2} \mathrm{~N}[\mathrm{M}+\mathrm{H}]^{+}: 296.1645$, found: 296.1649. Enantiomeric excess of the mixture of product was determined to be $98 \%(6 \mathbf{6}-\mathbf{A})$ and $87 \%(6 \mathbf{6}-\mathbf{B})$ ee by chiral stationary phase HPLC analysis (CHIRALPAK OD-3 $(\phi 0.46 \mathrm{~cm}$ x $25 \mathrm{~cm}), n$-hexane/2-propanol = 95/5, flow rate $1.0 \mathrm{~mL} / \mathrm{min}$, detection at $254 \mathrm{~nm}$. 6a-A $\mathrm{t}_{R}=16.96 \mathrm{~min}$ (minor), $17.65 \mathrm{~min}$ (major); $\mathbf{6 a}-\mathbf{B} \mathrm{t}_{R}=10.68 \mathrm{~min}$ (minor), $14.59 \min$ (major);
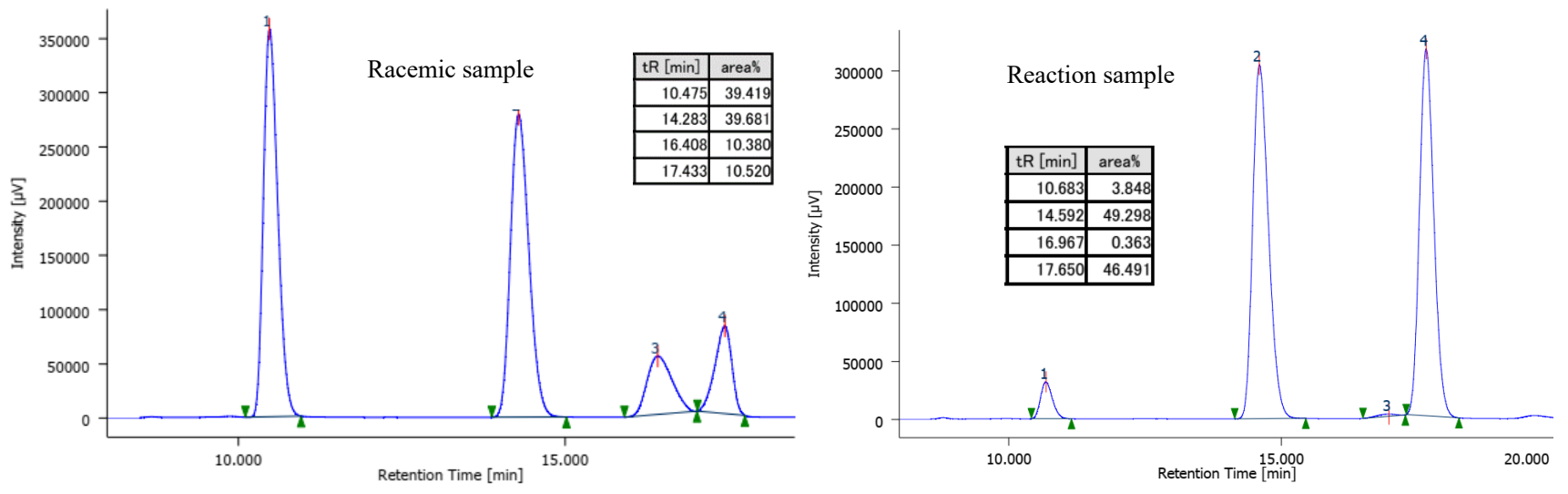

Mixture of methyl (R)-3-([1,1'-biphenyl]-4-ylmethyl)-1,2,3,4-tetrahydroquinoline-3-carboxylate (6b-A) and methyl $(R)-3$-benzyl-7-phenyl-1,2,3,4-tetrahydroquinoline-3-carboxylate (6b-B) 


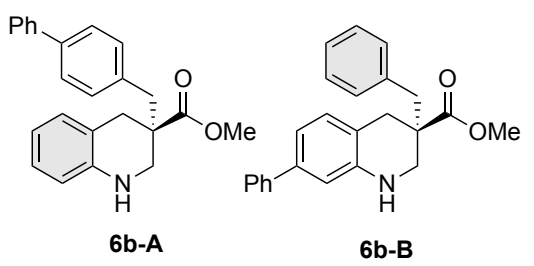

Prepared by the general procedure E from $3 \mathbf{b}(34.3 \mathrm{mg}, 0.1 \mathrm{mmol})$, stirred for $2 \mathrm{~h}$, and isolated by silica gel column chromatgraphy (hexane/ethyl acetate) as a 48:52 mixture (31.4 mg, 88\% yield). Colourless liquid; ${ }^{1}$ H NMR (400 MHz, $\left.\mathrm{CDCl}_{3}\right):{ }^{1} \mathrm{H} \mathrm{NMR}\left(400 \mathrm{MHz}, \mathrm{CDCl}_{3}\right) \delta$ 7.59-7.53 (m, $\left.4.7 \mathrm{H}\right), 7.51-7.48(\mathrm{~m}$, $1.93 \mathrm{H}), 7.45-7.38(\mathrm{~m}, 4 \mathrm{H}), 7.35-7.26(\mathrm{~m}, 4 \mathrm{H}), 7.25-7.20(\mathrm{~m}, 1 \mathrm{H}), 7.17-$ $7.15(\mathrm{~m}, 1.89 \mathrm{H}), 7.12-7.09(\mathrm{~m}, 2.12 \mathrm{H}), 7.07-7.00(\mathrm{~m}, 3 \mathrm{H}), 6.90(\mathrm{dd}, J=7.8,1.8 \mathrm{~Hz}, 1.07 \mathrm{H}), 6.75(\mathrm{~d}, J=1.7 \mathrm{~Hz}$, $1.07 \mathrm{H}), 6.67(\mathrm{td}, J=7.4,1.1 \mathrm{~Hz}, 0.93 \mathrm{H}), 6.55(\mathrm{dd}, J=8.4,1.0 \mathrm{~Hz}, 0.93 \mathrm{H}), 3.99$ (br, $1.07 \mathrm{H}), 3.93(\mathrm{br}, 0.93 \mathrm{H}), 3.67$ (s, 2.80H), $3.65(\mathrm{~s}, 3.20 \mathrm{H}), 3.53(\mathrm{dd}, J=7.6,0.9 \mathrm{~Hz}, 0.94 \mathrm{H}), 3.50(\mathrm{dd}, J=7.6,0.9 \mathrm{~Hz}, 1.06 \mathrm{H}), 3.26(\mathrm{~d}, J=11.6$ $\mathrm{Hz}, 2 \mathrm{H}), 3.13(\mathrm{~d}, J=16.3 \mathrm{~Hz}, 2 \mathrm{H}), 3.00(\mathrm{ddd}, J=19.1,13.4,4.6 \mathrm{~Hz}, 4 \mathrm{H}), 2.85(\mathrm{~d}, J=16.4 \mathrm{~Hz}, 2 \mathrm{H})$; ${ }^{13} \mathbf{C} \mathbf{N M R}$ $\left(101 \mathrm{MHz}, \mathrm{CDCl}_{3}\right): \delta 175.2,175.1,143.5,143.2,141.4,140.9,140.2,139.6,136.9,136.1,130.3,130.2,129.9$, $129.8,128.7,128.6,128.2$, 127.2, 127.1, 127.0, 127.0, 127.0, 126.9, 126.8, 119.0, 118.3, 117.7, 116.8, 114.1, 112.7, 51.8, 47.1, 46.9, 45.8, 45.7, 41.4, 40.9, 34.9, 34.6; IR (thin film): 3406, 3026, 2948, 2839, 1725, 1607, 1486, 1437 , 1363, 1197, 1077, 849, $757 \mathrm{~cm}^{-1}$; HRMS (ESI) $\mathrm{m} / z$ calc'd for $\mathrm{C}_{24} \mathrm{H}_{24} \mathrm{O}_{2} \mathrm{~N}[\mathrm{M}+\mathrm{H}]^{+}: 358.1802$, found: 358.1804 ; Enantiomeric excess of the mixture of product was determined to be $89 \%(\mathbf{6 b}-\mathbf{A})$ and $96 \%(6 \mathbf{b}-\mathbf{B})$ ee by chiral stationary phase HPLC analysis (CHIRALPAK OJH $(\phi 0.46 \mathrm{~cm}$ x $25 \mathrm{~cm}), n$-hexane/2-propanol = 95/5, flow rate $1.0 \mathrm{~mL} / \mathrm{min}$, detection at $254 \mathrm{~nm}$. 6b-A $\mathrm{t}_{R}=24.05 \mathrm{~min}$ (minor), $30.27 \mathrm{~min}$ (major); $\mathbf{6 b}-\mathbf{B} \mathrm{t}_{R}=12.92 \min$ (minor), $17.81 \mathrm{~min}$ (major).
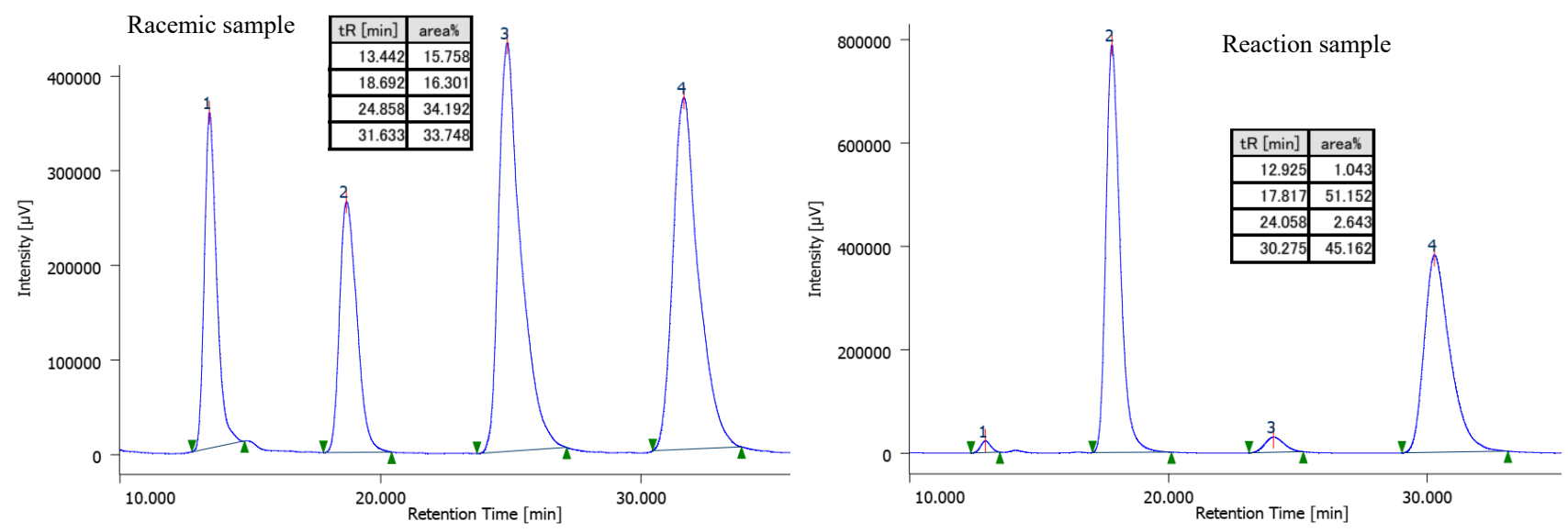

Mixture of methyl (R)-3-(2-methylbenzyl)-1,2,3,4-tetrahydroquinoline-3-carboxylate (6c-A) and methyl $(R)$ 3-benzyl-5-methyl-1,2,3,4-tetrahydroquinoline-3-carboxylate (6c-B)

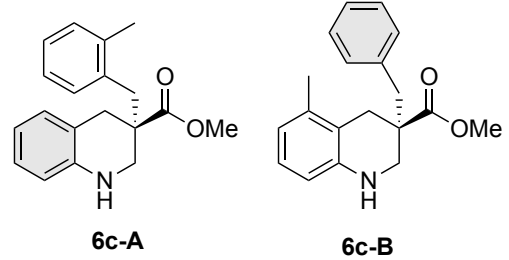

Prepared by the general procedure E from 3c $(28.1 \mathrm{mg}, 0.1 \mathrm{mmol})$, stirred for 5 $\mathrm{h}$, and isolated by silica gel column chromatgraphy (hexane/ethyl acetate) as a 53:47 mixture (25.0 mg, 85\% yield). White solid; m.p. $119-121{ }^{\circ} \mathrm{C} ;{ }^{1} \mathbf{H}$ NMR $\left(400 \mathrm{MHz}, \mathrm{CDCl}_{3}\right): \delta 7.28-7.26(\mathrm{~m}, 1 \mathrm{H}), 7.24-7.20(\mathrm{~m}, 2 \mathrm{H}), 7.14-7.07(\mathrm{~m}$, $5 \mathrm{H}), 7.05-7.03(\mathrm{~m}, 1 \mathrm{H}), 6.98(\mathrm{t}, J=7.9 \mathrm{~Hz}, 2 \mathrm{H}), 6.92(\mathrm{t}, J=7.7 \mathrm{~Hz}, 1 \mathrm{H})$, $6.64(\mathrm{td}, J=7.5,0.9 \mathrm{~Hz}, 0.94 \mathrm{H}), 6.56(\mathrm{~d}, J=7.3 \mathrm{~Hz}, 1.04 \mathrm{H}), 6.50(\mathrm{~d}, J=7.9 \mathrm{~Hz}, 0.94 \mathrm{H}), 6.42(\mathrm{~d}, J=8.0 \mathrm{~Hz}$, 
$1.04 \mathrm{H}), 3.87(\mathrm{br}, 2 \mathrm{H}), 3.65(\mathrm{~s}, 3.13 \mathrm{H}), 3.60(\mathrm{~s}, 2.87 \mathrm{H}), 3.54(\mathrm{dd}, J=11.6,1.5 \mathrm{~Hz}, 1 \mathrm{H}), 3.43(\mathrm{~d}, J=11.5 \mathrm{~Hz}, 1 \mathrm{H})$, $3.28(\mathrm{~d}, J=11.5 \mathrm{~Hz}, 1 \mathrm{H}), 3.20(\mathrm{dd}, J=11.5,1.5 \mathrm{~Hz}, 1 \mathrm{H}), 3.13(\mathrm{~d}, J=16.2 \mathrm{~Hz}, 1 \mathrm{H}), 3.06-2.99(\mathrm{~m}, 3 \mathrm{H}), 2.92(\mathrm{~d}, J=$ 13.6 Hz, 2H), $2.83(\mathrm{~d}, J=16.3 \mathrm{~Hz}, 1 \mathrm{H}), 2.70(\mathrm{~d}, J=16.7 \mathrm{~Hz}, 1 \mathrm{H}), 2.23(\mathrm{~s}, 2.87 \mathrm{H}), 2.18(\mathrm{~s}, 3.13 \mathrm{H}) ;{ }^{13} \mathbf{C}$ NMR $(101$ $\left.\mathrm{MHz}, \mathrm{CDCl}_{3}\right): \delta 175.5,175.4,143.3,143.1,137.6,137.2,137.0,135.2,130.5,129.9,129.8,128.2,127.0,126.7$, 126.5, 125.6, 119.4, 119.4, 117.8, 117.7, 114.1, 112.2, 51.9, 51.8, 47.7, 46.3, 46.1, 45.8, 41.4, 38.0, 34.8, 32.1, 19.8, 19.5; IR (thin film): 3407, 3025, 2948, 2855, 1725, 1590, 1494, 1435, 1361, 1282, 1197, 1082, 865, $744 \mathrm{~cm}^{-1}$; HRMS (ESI) $m / z$ calc'd for $\mathrm{C}_{19} \mathrm{H}_{22} \mathrm{O}_{2} \mathrm{~N}[\mathrm{M}+\mathrm{H}]^{+}: 296.1645$, found: 296.1648 . Enantiomeric excess of the mixture of product was determined to be $78 \%$ (6c-A) and 95\% (6c-B) ee by chiral stationary phase HPLC analysis (CHIRALPAK IA $(\phi 0.46 \mathrm{~cm} \times 25 \mathrm{~cm}), n$-hexane $/ 2$-propanol $=90 / 10$, flow rate $1.0 \mathrm{~mL} / \mathrm{min}$, detection at $254 \mathrm{~nm}$. 6c-A $\mathrm{t}_{R}=7.24 \mathrm{~min}$ (major), $10.20 \mathrm{~min}$ (minor); $\mathbf{6 c}-\mathbf{B} \mathrm{t}_{R}=8.77 \mathrm{~min}$ (major), $19.70 \mathrm{~min}$ (minor).
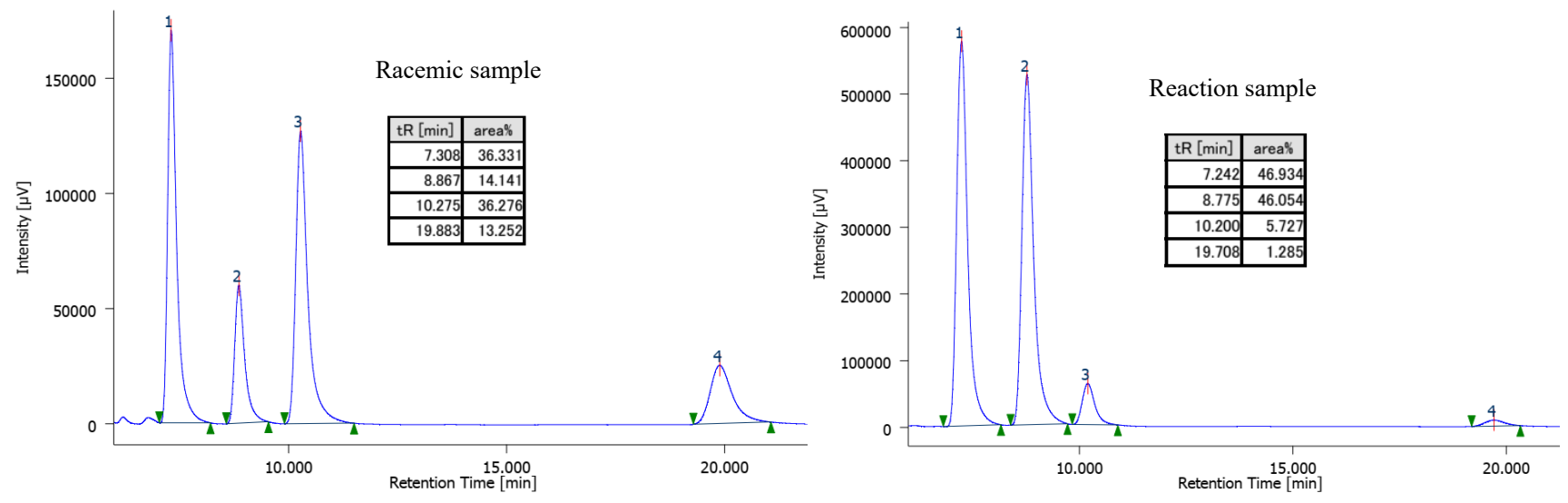

Mixture of methyl (R)-7-methyl-3-(2-methylbenzyl)-1,2,3,4-tetrahydroquinoline-3-carboxylate (6d-A) and methyl $(R)-5$-methyl-3-(4-methylbenzyl)-1,2,3,4-tetrahydroquinoline-3-carboxylate (6d-B)

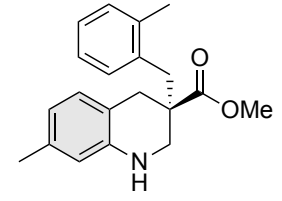

6d-A

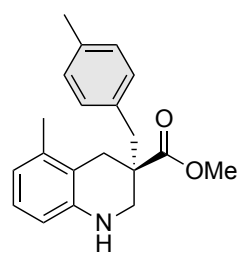

$6 d-B$

Prepared by the general procedure E from 3d $(29.5 \mathrm{mg}, 0.1 \mathrm{mmol})$, stirred for $5 \mathrm{~h}$, and isolated by silica gel column chromatgraphy (hexane/ethyl acetate) as a 50:50 mixture (25.9 mg, 84\% yield). Colorless oil; ${ }^{1} \mathbf{H}$ NMR (400 MHz, $\left.\mathrm{CDCl}_{3}\right): \delta 7.13-7.02(\mathrm{~m}, 6 \mathrm{H}), 6.97(\mathrm{~d}, J=8.0 \mathrm{~Hz}, 2 \mathrm{H}), 6.91$ (t, $J=7.7 \mathrm{~Hz}$, $1 \mathrm{H}), 6.85$ (d, $J=7.6 \mathrm{~Hz}, 1 \mathrm{H}), 6.55(\mathrm{~d}, J=7.3 \mathrm{~Hz}, 1 \mathrm{H}), 6.46$ (dd, $J=7.6,1.0$ $\mathrm{Hz}, 1 \mathrm{H}), 6.41$ (d, $J=7.9 \mathrm{~Hz}, 1 \mathrm{H}), 6.33(\mathrm{~s}, 1 \mathrm{H}), 3.65$ (s, 3H), 3.59 (s, 3H), 3.52 (dd, $J=11.5,1.6 \mathrm{~Hz}, 1 \mathrm{H}), 3.41$ (dd, $J=11.4,0.9 \mathrm{~Hz}, 1 \mathrm{H}), 3.25(\mathrm{dd}, J=11.5,1.2 \mathrm{~Hz}, 1 \mathrm{H}), 3.18(\mathrm{dd}, J=11.5,1.7 \mathrm{~Hz}, 1 \mathrm{H}), 3.09(\mathrm{~d}, J=16.1 \mathrm{~Hz}, 1 \mathrm{H})$, $3.01-2.77(\mathrm{~m}, 5 \mathrm{H}), 2.79(\mathrm{~d}, J=16.1 \mathrm{~Hz}, 1 \mathrm{H}), 2.69(\mathrm{~d}, J=16.6 \mathrm{~Hz}, 1 \mathrm{H}), 2.31(\mathrm{~s}, 3 \mathrm{H}), 2.23(\mathrm{~s}, 3 \mathrm{H}), 2.21(\mathrm{~s}, 3 \mathrm{H})$, 2.17 (s, 3H); ${ }^{13} \mathbf{C}$ NMR (101 MHz, $\left.\mathrm{CDCl}_{3}\right): \delta$ 175.5, 175.5, 143.3, 143.0, 137.6, 137.0, 136.7, 136.2, 135.3, 134.0, $130.5,130.5,129.7,129.7,128.9,126.7,126.5,125.6,119.4,118.7,117.8,116.4,114.7,112.2,51.8,51.8,47.7$, 46.4, 46.1, 46.0, 41.0, 38.0, 34.5, 32.1, 21.2, 21.1, 19.8, 19.5; IR (thin film): 3405, 3015, 2947, 2855, 1720, 1617, 1584, 1491, 1432, 1360, 1285, 1197, 1111, 1032, 865, $742 \mathrm{~cm}^{-1}$; HRMS (ESI) $m / z$ calc'd for $\mathrm{C}_{20} \mathrm{H}_{24} \mathrm{O}_{2} \mathrm{~N}[\mathrm{M}+\mathrm{H}]^{+}$: 310.1802, found: 310.1803 . Enantiomeric excess of the mixture of product was determined to be $89 \%(\mathbf{6 d - A})$ and 
90\% (6d-B) ee by chiral stationary phase HPLC analysis (CHIRALPAK IA $(\phi 0.46 \mathrm{~cm} \times 25 \mathrm{~cm}), n$-hexane/2propanol $=90 / 10$, flow rate $1.0 \mathrm{~mL} / \mathrm{min}$, detection at $254 \mathrm{~nm}$. 6d-A $\mathrm{t}_{R}=7.61 \mathrm{~min}$ (major), $11.53 \mathrm{~min}$ (minor); $6 \mathbf{d}-\mathbf{B}$ $\mathrm{t}_{R}=9.13 \min$ (major), $21.76 \min$ (minor).
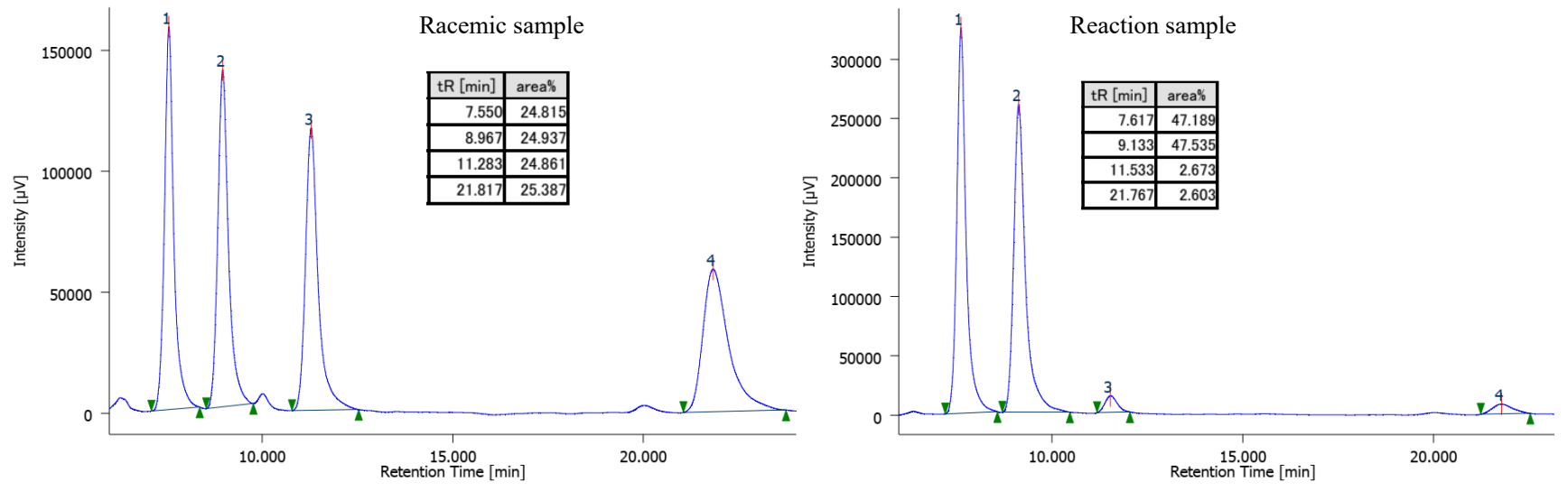

Mixture of methyl (R)-3-(4-bromobenzyl)-7-chloro-1,2,3,4-tetrahydroquinoline-3-carboxylate (6e-A) and methyl $(R)$-7-bromo-3-(4-chlorobenzyl)-1,2,3,4-tetrahydroquinoline-3-carboxylate (6e-B)

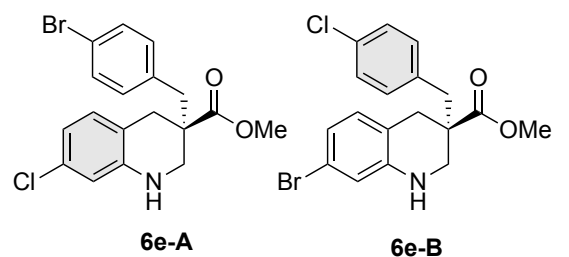

Prepared by the general procedure E from 3 e $(37.8 \mathrm{mg}, 0.1 \mathrm{mmol})$, stirred for $7 \mathrm{~h}$, and isolated by silica gel column chromatgraphy (hexane/ethyl acetate) as a $49: 51$ mixture (18.4 mg, 47\% yield). White solid; m.p. $124-126{ }^{\circ} \mathrm{C} ;{ }^{1} \mathbf{H}$ NMR (400 MHz, $\left.\mathrm{CDCl}_{3}\right): \delta 7.40-7.37(\mathrm{~m}, 2 \mathrm{H}), 7.24-7.22(\mathrm{~m}, 2 \mathrm{H}), 7.02-$ $6.98(\mathrm{~m}, 2 \mathrm{H}), 6.95-6.92(\mathrm{~m}, 2 \mathrm{H}), 6.87(\mathrm{~d}, J=8.1 \mathrm{~Hz}, 1 \mathrm{H}), 6.82(\mathrm{~d}, J=8.0$ $\mathrm{Hz}, 1 \mathrm{H}), 6.75(\mathrm{dd}, J=8.0,1.9 \mathrm{~Hz}, 1 \mathrm{H}), 6.66(\mathrm{~d}, J=1.9 \mathrm{~Hz}, 1.08 \mathrm{H}), 6.61(\mathrm{dd}, J=8.0,2.0 \mathrm{~Hz}, 0.93 \mathrm{H}), 6.51(\mathrm{~d}, J=$ $2.0 \mathrm{~Hz}, 0.86 \mathrm{H}), 3.97$ (br, 2H), 3.63 (br, 6H), 3.45 (dd, $J=11.7,0.9 \mathrm{~Hz}, 2 \mathrm{H}), 3.18$ (dd, J=11.7, $1.5 \mathrm{~Hz}, 2 \mathrm{H}), 3.02$ $(\mathrm{d}, J=6.5 \mathrm{~Hz}, 1 \mathrm{H}), 2.97(\mathrm{dd}, J=11.6,5.8 \mathrm{~Hz}, 2 \mathrm{H}), 2.92(\mathrm{~d}, J=5.0 \mathrm{~Hz}, 1 \mathrm{H}), 2.86-2.81(\mathrm{~m}, 2 \mathrm{H}), 2.73(\mathrm{~d}, J=5.6 \mathrm{~Hz}$, 1H), $2.70-2.68(\mathrm{~m}, 1 \mathrm{H}) ;{ }^{13} \mathbf{C}$ NMR (101 $\left.\mathrm{MHz}_{\mathrm{CDCl}}\right): \delta$ 174.6, 174.6, 144.3, 144.0, 135.7, 135.2, 132.8, 132.5, 131.5, 131.4, 131.2, 131.1, 130.9, 128.4, 120.9, 120.5, 120.4, 117.6, 117.6, 117.1, 116.4, 113.5, 52.0, 46.6, 45.4, 40.4, 40.4, 34.4, 34.3; IR (thin film): 3407, 2948, 2840, 1720, 1598, 1487, 1282, 1195, 1176, 1071, 1011, 839, 783 $\mathrm{cm}^{-1}$; HRMS (ESI) $\mathrm{m} / z$ calc'd for $\mathrm{C}_{18} \mathrm{H}_{18} \mathrm{O}_{2} \mathrm{NBrCl}[\mathrm{M}+\mathrm{H}]^{+}:$394.0204, found: 394.0203. Enantiomeric excess of the mixture of product was determined to be $68 \%(\mathbf{6 e - A})$ and $89 \%(6 \mathbf{e}-\mathbf{B})$ ee by chiral stationary phase HPLC analysis (CHIRALPAK OD ( $\phi 0.46 \mathrm{~cm} \times 25 \mathrm{~cm}), n$-hexane $/ 2$-propanol $=95 / 5$, flow rate $1.0 \mathrm{~mL} / \mathrm{min}$, detection at $254 \mathrm{~nm}$. 6e-A $\mathrm{t}_{R}=15.43 \mathrm{~min}$ (minor), $19.18 \mathrm{~min}$ (major); 6e-B $\mathrm{t}_{R}=14.38 \mathrm{~min}$ (minor), 17.31min (major). 

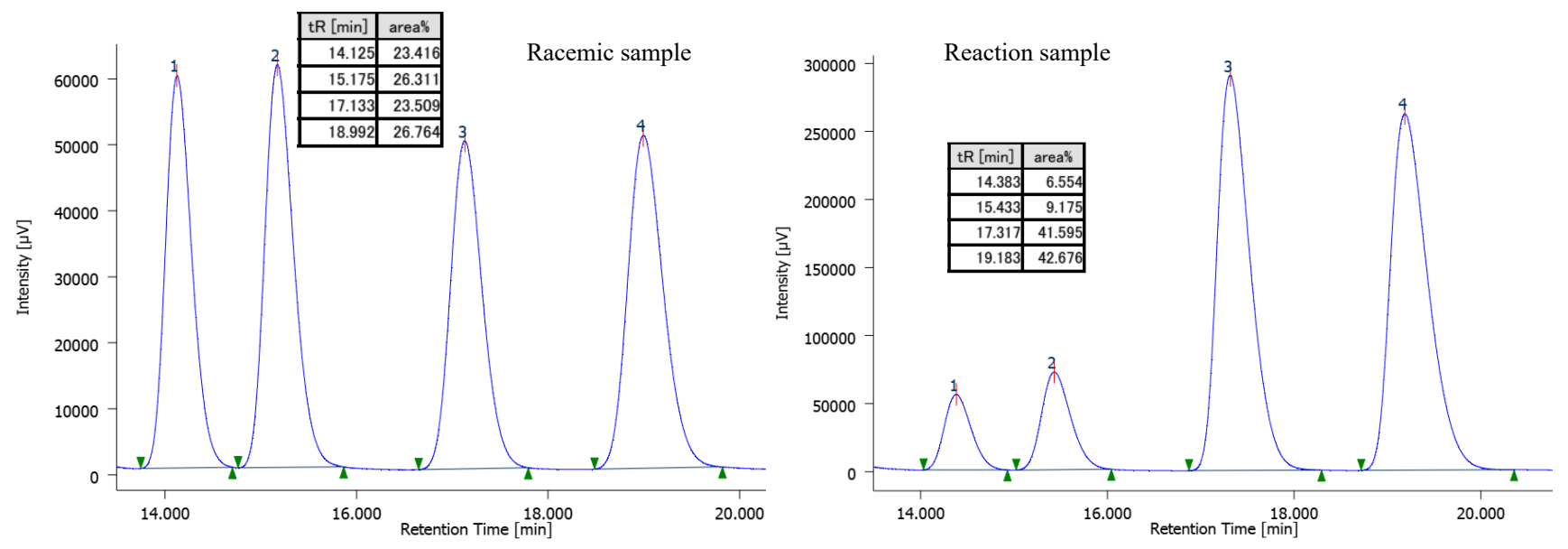

\section{4-3. Kinetic resolution}

\section{4-3-1. Kinetic study}

The kinetic study was conducted under synthetically relevant conditions $(0.05-0.15 \mathrm{M}$ initial substrate concentrations, 2.2-6.6 mol\% catalyst loadings) to evaluate the copper complex and the substrate dependencies on the reaction rate. The reaction progression with varied initial concentrations were monitored over the course of the reaction. Only substrate 1a and product $\mathbf{2 a}$ were observed, and no intermediate was detected in ${ }^{1} \mathrm{H}-\mathrm{NMR}$ analyses. Four series of experiments were conducted, and each series comprise five data points.

$\begin{array}{ccc}\text { Entry } & \text { Substrate concentration }(\mathrm{M}) & \text { Catalyst concentration }(\mathrm{M}) \\ 1 & 0.10 & 0.0033 \\ 2 & 0.05 & 0.0033 \\ 3 & 0.15 & 0.0033 \\ 4 & 0.10 & 0.01\end{array}$

\section{Substrate order:}

Solutions of substrate 1a (0.2 M in HFIP) and copper complex (0.0167 M in HFIP, 1:1 CuBr/L1) were prepared. To a flame-dried $10 \mathrm{~mL}$ test tube equipped with a magnetic stirring bar were added a solution of 1a and HFIP, followed by the addition of a solution of the copper at $0{ }^{\circ} \mathrm{C}$. The reaction mixture was quenched with aq EDTA and water. The aqueous layer was extracted with EtOAc $(3 \mathrm{x})$. The combined organic layers were concentrated under reduced pressure. The yields were determined by crude ${ }^{1} \mathrm{H}-\mathrm{NMR}$ analysis using 1,1,2,2-tetrachloroethane as an internal standard.

\begin{tabular}{ccccccc} 
catalyst loading & \multicolumn{1}{c}{$\mathbf{1 a}(0.2 \mathrm{M}$ in HFIP $)$} & $\mathrm{Cu} / \mathbf{L} \mathbf{1}(0.0167 \mathrm{M}$ in HFIP $)$ & HFIP & Total volume \\
$3.3 \mathrm{~mol} \%$ & $500 \mu \mathrm{L}$ & $0.1 \mathrm{M}$ & $200 \mu \mathrm{L}$ & $0.0033 \mathrm{M}$ & $300 \mu \mathrm{L}$ & $1000 \mu \mathrm{L}$ \\
$6.6 \mathrm{~mol} \%$ & $250 \mu \mathrm{L}$ & $0.05 \mathrm{M}$ & $200 \mu \mathrm{L}$ & $0.0033 \mathrm{M}$ & $550 \mu \mathrm{L}$ & $1000 \mu \mathrm{L}$ \\
$2.2 \mathrm{~mol} \%$ & $750 \mu \mathrm{L}$ & $0.15 \mathrm{M}$ & $200 \mu \mathrm{L}$ & $0.0033 \mathrm{M}$ & $50 \mu \mathrm{L}$ & $1000 \mu \mathrm{L}$
\end{tabular}


The following plots show the consumption of $\mathbf{1 a}$ and the evolution of $\mathbf{2 a}$ over the reaction.
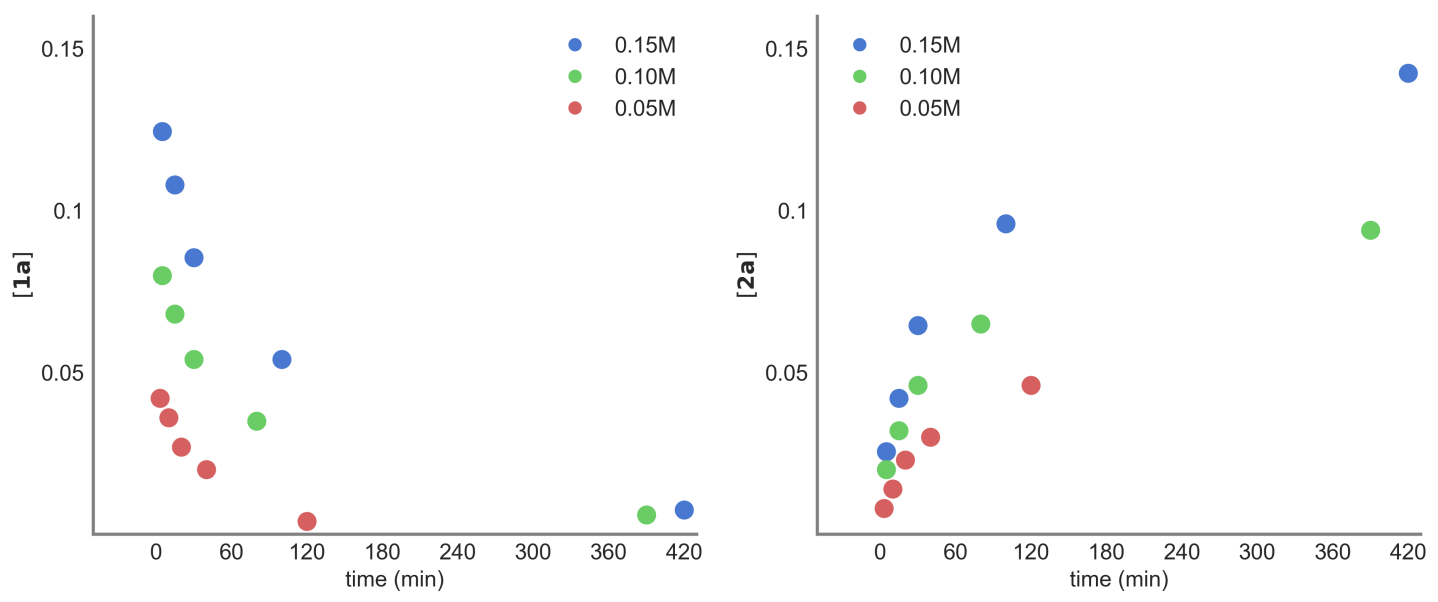

The $\mathrm{k}_{\mathrm{obs}}$ obtained from the data are shown below. The results reveals that the values of $\mathrm{k}_{\mathrm{obs}}$ do not depend on the substrate concentration $\left(0.007^{\text {th }}\right.$ order $)$.
Substrate concentration
0.05
0.1
0.15
$\mathrm{k}_{\mathrm{obs}}$
0.00088
0.00103
0.00156

\section{Catalyst order:}

In a similar manner to the procedure above, another series (entry 4) of experiments was performed. The following plots show the consumption of $\mathbf{1 a}$ and the evolution of $\mathbf{2 a}$ over the reaction with 3.3 and $10 \mathrm{~mol} \%$ catalyst loadings and $1 \mathrm{a}$ initial concentration $0.1 \mathrm{M}$.
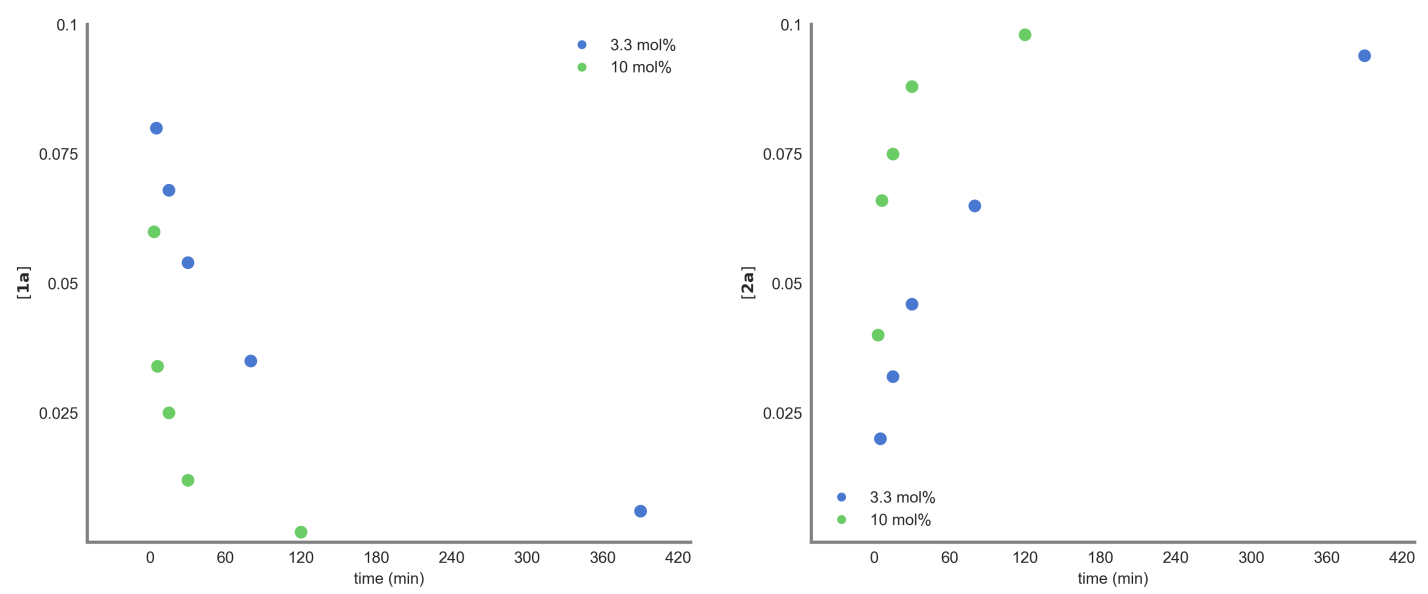

To evaluate the order of copper on the rate, the normalized time scale method developed by Burés was used. ${ }^{4}$ The visual inspection of the plots shows the second order dependency for the copper/L1 concentration, which is in line 
with our previous observation on the $\mathrm{Cu} / \mathrm{BOX}$ catalytic system. Therefore, the active intermediate of $\mathrm{CuBr} / \mathbf{L 1}$ is also likely a dicopper nitrene.
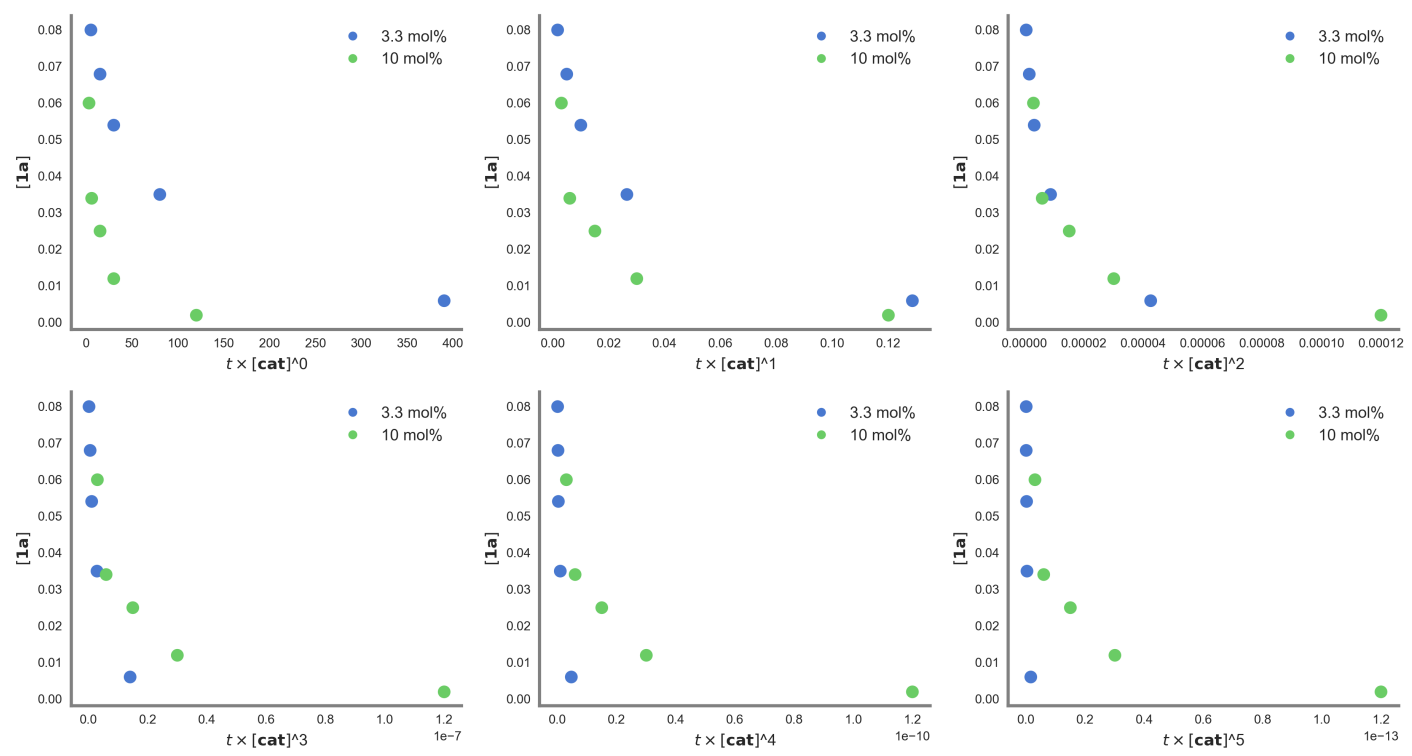

\section{4-3-2. Kinetic resolution}

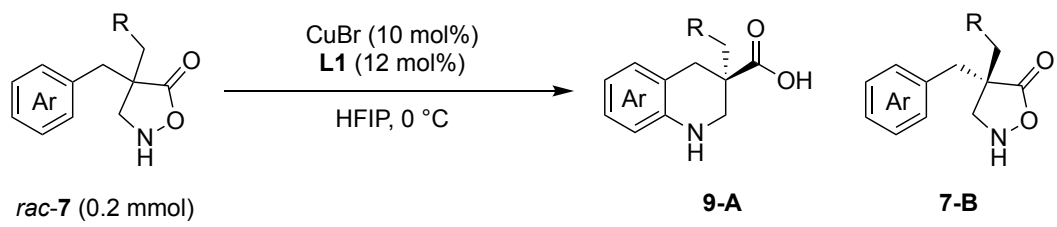

General procedure F: To a flame-dried $10 \mathrm{~mL}$ test tube equipped with a magnetic stirring bar were added $\mathbf{L 1}$ (5.7 $\mathrm{mg}, 0.024 \mathrm{mmol}, 12 \mathrm{~mol} \%)$ and $\mathrm{CuBr}(2.9 \mathrm{mg}, 0.020 \mathrm{mmol}, 10 \mathrm{~mol} \%)$ in glove box, followed by the addition of HFIP $(1.0 \mathrm{~mL})$ and the mixture was stirred for $15 \mathrm{~min}$ at an ambient temperature. To the solution cooled at $0{ }^{\circ} \mathrm{C}$ and substrate $7(0.20 \mathrm{mmol})$ dissolved in $1.0 \mathrm{~mL}$ HFIP was added. The reaction mixture was stirred for the indicated time at the same temperature and was quenched by the addition of aq EDTA and water. The aqueous layer was extracted with EtOAc $(3 \mathrm{x})$. The combined organic layers were concentrated under reduced pressure. Unreacted substrate 7-B and cyclized acid 9-A were separated by the PTLC (40\% hexane : EtOAc with 1\% MeOH). [Note: The scratched silica powder containing the acid compound was washed with $50 \% \mathrm{MeOH}$ : EtOAc].

The obtained acid was dissolved in 9:1 toluene:MeOH $(1.0 \mathrm{~mL}, 0.1 \mathrm{M})$. To the solution cooled with an ice bath was added (trimethylsilyl)diazomethane $\left(2.0 \mathrm{M}\right.$ in $\mathrm{Et}_{2} \mathrm{O}, 75 \mu \mathrm{L}, 1.5$ equiv). After 15 min, the reaction was quenched by the addition of aq $\mathrm{AcOH}$, followed by sat aq $\mathrm{NaHCO}_{3}$. The resulting aqueous layer was extracted with 
EtOAc (3x). The combined organic layers were concentrated under reduced pressure to give the crude product, which was purified by silica gel column chromatography to afford 9-A methyl ester.

\section{(S)-4-Benzyl-4-(cyclohexylmethyl)isoxazolidin-5-one (7a-B)}

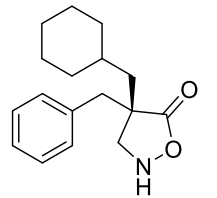

Prepared by the general procedure F from $7 \mathbf{a}(54.6 \mathrm{mg}, 0.2 \mathrm{mmol})$ and NMR yield was $49 \%$ and isolated by PTLC (40\% hexane : EtOAc with $1 \% \mathrm{MeOH})$ as a white solid (24.5 $\mathrm{mg}, 45 \%$ yield). $[\alpha]_{\mathrm{D}}^{27}+49.75$ (c 1.0, $\mathrm{CHCl}_{3}, 92.3 \%$ ee sample).

Conversion 51\%; Selectivity factor $(s) 55$; Enantiomeric excess of the product was determined to be $92.3 \%$ ee by chiral stationary phase HPLC analysis (CHIRALPAK IA $(\phi 0.46 \mathrm{~cm} \times 25 \mathrm{~cm}), n$-hexane/2propanol $=80 / 20$, flow rate $0.8 \mathrm{~mL} / \mathrm{min}$, detection at $254 \mathrm{~nm}, \mathrm{t}_{R}=11.11 \mathrm{~min}$ (major), $14.96 \mathrm{~min}$ (minor).
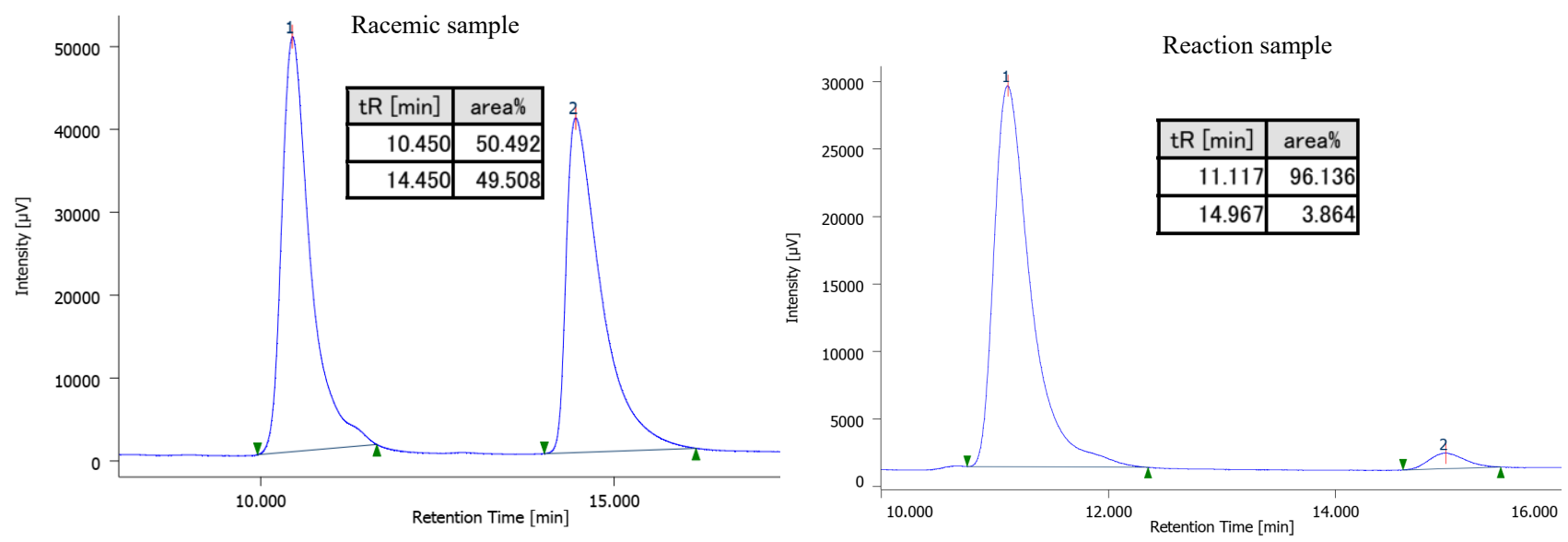

\section{Methyl (R)-3-(cyclohexylmethyl)-1,2,3,4-tetrahydroquinoline-3-carboxylate (9a-A methyl ester)}

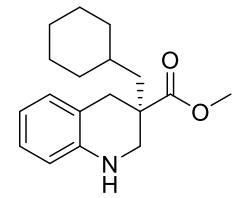

Prepared by the general procedure F from $7 \mathbf{a}(54.6 \mathrm{mg}, 0.2 \mathrm{mmol})$ and NMR yield of 9a-A was $50 \%$ and estrification compound was isolated by silica gel column chromatgraphy (hexane/ethyl acetate) as a white solid (25.8 mg, 45\% yield). m.p. $57-59{ }^{\circ} \mathrm{C} ;{ }^{1} \mathbf{H}$ NMR (400 MHz, $\left.\mathrm{CDCl}_{3}\right): \delta$ 6.98-6.94 (m, 2H), 6.64 (td, $J=7.4,1.1 \mathrm{~Hz}, 1 \mathrm{H}), 6.48$ (d, $J=7.9 \mathrm{~Hz}, 1 \mathrm{H}), 3.81$ (br, 1H), 3.64 (s, 3H), 3.49 (dd, $J=11.5,1.5 \mathrm{~Hz}, 1 \mathrm{H}), 3.16$ (t, $J=14.2 \mathrm{~Hz}, 2 \mathrm{H}), 2.68$ (d, $J=16.1 \mathrm{~Hz}, 1 \mathrm{H}), 1.66-1.59(\mathrm{~m}, 6 \mathrm{H}), 1.50$ - $1.37(\mathrm{~m}, 2 \mathrm{H}), 1.28-1.05(\mathrm{~m}, 3 \mathrm{H}), 0.95-0.84(\mathrm{~m}, 2 \mathrm{H}) ;{ }^{13} \mathbf{C} \mathbf{N M R}\left(101 \mathrm{MHz}, \mathrm{CDCl}_{3}\right): \delta$ 176.4, 143.5, 129.7, $126.8,119.8,117.6,114.1,51.8,48.7,43.7,43.6,35.7,34.5,34.1,33.8,26.4,26.3,26.2$; IR (thin film): 3408, 2922, 2850, 1729, 1608, 1504, 1447, 1283, 1208, 1031, 894, $746 \mathrm{~cm}^{-1}$; HRMS (ESI) $m / z$ calc'd for $\mathrm{C}_{18} \mathrm{H}_{26} \mathrm{O}_{2} \mathrm{~N}[\mathrm{M}+\mathrm{H}]^{+}$: 288.1958, found: $288.1959 ;[\alpha]_{\mathrm{D}}^{27}+51.30\left(c 1.0, \mathrm{CHCl}_{3}, 84.2 \%\right.$ ee sample). Enantiomeric excess of the product was determined to be $84.2 \%$ ee by chiral stationary phase HPLC analysis (CHIRALPAK IA $(\phi 0.46 \mathrm{~cm} \mathrm{x} 25 \mathrm{~cm})$, $n$-hexane/2-propanol $=80 / 20$, flow rate $1.0 \mathrm{~mL} / \mathrm{min}$, detection at $254 \mathrm{~nm}, \mathrm{t}_{R}=6.45 \mathrm{~min}$ (major), $12.65 \min$ (minor). 

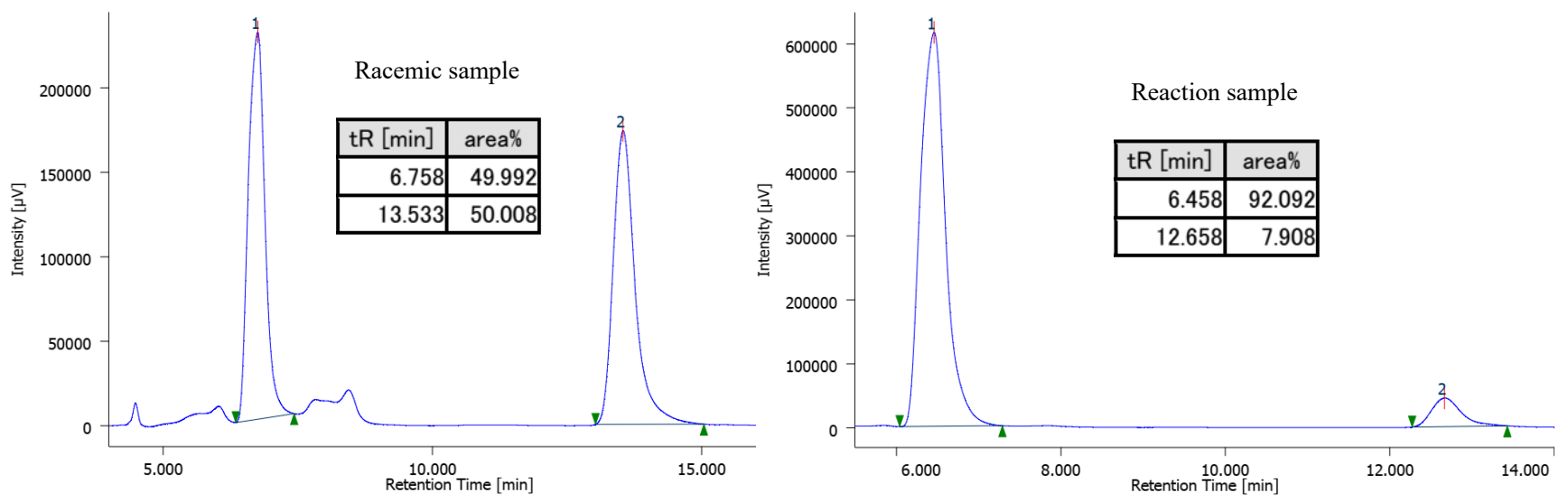

(S)-4-(Cyclohexylmethyl)-4-(4-methylbenzyl)isoxazolidin-5-one (7b-B)

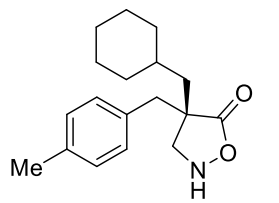

Prepared by the general procedure F from $7 \mathbf{b}(57.4 \mathrm{mg}, 0.2 \mathrm{mmol})$ and NMR yield was $58 \%$ and isolated by PTLC (40\% hexane : EtOAc with $1 \% \mathrm{MeOH})$ as a white solid (31.5 $\mathrm{mg}, 55 \%$ yield). $[\alpha]_{\mathrm{D}}^{27}+55.0$ (c 1.0, $\mathrm{CHCl}_{3}, 61 \%$ ee sample). Conversion $42 \%$; Selectivity factor $(s) 22$. Enantiomeric excess of the product was determined to be $61 \%$ ee by chiral stationary phase HPLC analysis (CHIRALPAK IC ( $\phi 0.46 \mathrm{~cm} \times 25 \mathrm{~cm}), n$-hexane $/ 2$-propanol $=80 / 20$, flow rate $0.8 \mathrm{~mL} / \mathrm{min}$, detection at $254 \mathrm{~nm}, \mathrm{t}_{R}=12.25 \min$ (major), $16.30 \mathrm{~min}$ (minor).
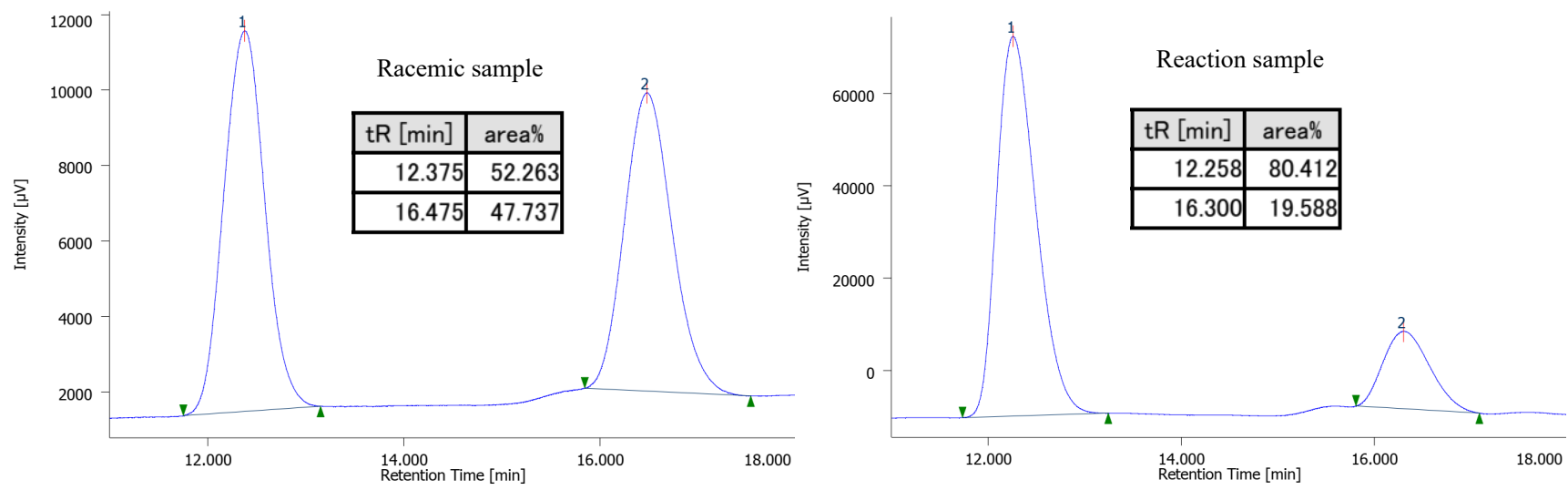

\section{Methyl (R)-3-(cyclohexylmethyl)-7-methyl-1,2,3,4-tetrahydroquinoline-3-carboxylate (9b-A methyl ester)}

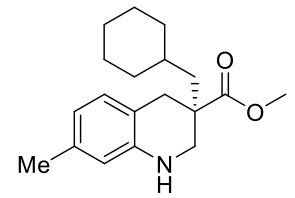

Prepared by the general procedure F from $7 \mathbf{b}(57.4 \mathrm{mg}, 0.2 \mathrm{mmol})$ and NMR yield of $\mathbf{9 b - A}$ was $42 \%$ and estrification compound was isolated by silica gel column chromatgraphy (hexane/ethyl acetate) as a white solid (24.0 mg, 40\% yield). m.p. 82-84 ${ }^{\circ} \mathrm{C} ;{ }^{1} \mathbf{H}$ NMR (400 $\left.\mathrm{MHz}, \mathrm{CDCl}_{3}\right): \delta 6.87(\mathrm{~d}, J=7.6 \mathrm{~Hz}, 1 \mathrm{H}), 6.47(\mathrm{~d}, J=7.6 \mathrm{~Hz}, 1 \mathrm{H}), 6.31(\mathrm{~s}, 1 \mathrm{H}), 3.75(\mathrm{br}, 1 \mathrm{H})$, $3.65(\mathrm{~s}, 3 \mathrm{H}), 3.47(\mathrm{dd}, J=11.5,1.5 \mathrm{~Hz}, 1 \mathrm{H}), 3.17-3.12(\mathrm{~m}, 2 \mathrm{H}), 2.64(\mathrm{~d}, J=16.1 \mathrm{~Hz}, 1 \mathrm{H}), 2.21(\mathrm{~s}, 3 \mathrm{H}), 1.66-$ $1.59(\mathrm{~m}, 6 \mathrm{H}), 1.49-1.40(\mathrm{~m}, 2 \mathrm{H}), 1.27-1.11(\mathrm{~m}, 3 \mathrm{H}), 0.95-0.84(\mathrm{~m}, 2 \mathrm{H}) ;{ }^{13} \mathbf{C} \mathbf{N M R}\left(101 \mathrm{MHz}, \mathrm{CDCl}_{3}\right): \delta 176.5$, 143.3, 136.4, 129.5, 118.7, 116.8, 114.7, 51.8, 48.7, 43.8, 43.5, 35.4, 34.5, 34.1, 33.8, 26.4, 26.3, 26.2, 21.1; IR (thin film): 3384, 2917, 2850, 1717, 1617, 1582, 1508, 1448, 1289, 1195, 1081, 977, 889, 853, $789 \mathrm{~cm}^{-1}$; HRMS 
(ESI) $m / z$ calc'd for $\mathrm{C}_{19} \mathrm{H}_{28} \mathrm{O}_{2} \mathrm{~N}[\mathrm{M}+\mathrm{H}]^{+}: 302.2115$, found: 302.2114 . $[\alpha]_{\mathrm{D}}{ }^{27}+33.27$ (c 1.0, $\mathrm{CHCl}_{3}, 82.4 \%$ ee sample). Enantiomeric excess of the product was determined to be $82.4 \%$ ee by chiral stationary phase HPLC analysis (CHIRALPAK IA $(\phi 0.46 \mathrm{~cm} \times 25 \mathrm{~cm}), n$-hexane $/ 2$-propanol $=90 / 10$, flow rate $1.0 \mathrm{~mL} / \mathrm{min}$, detection at $254 \mathrm{~nm}, \mathrm{t}_{R}=6.00 \mathrm{~min}$ (major), $12.78 \mathrm{~min}$ (minor).
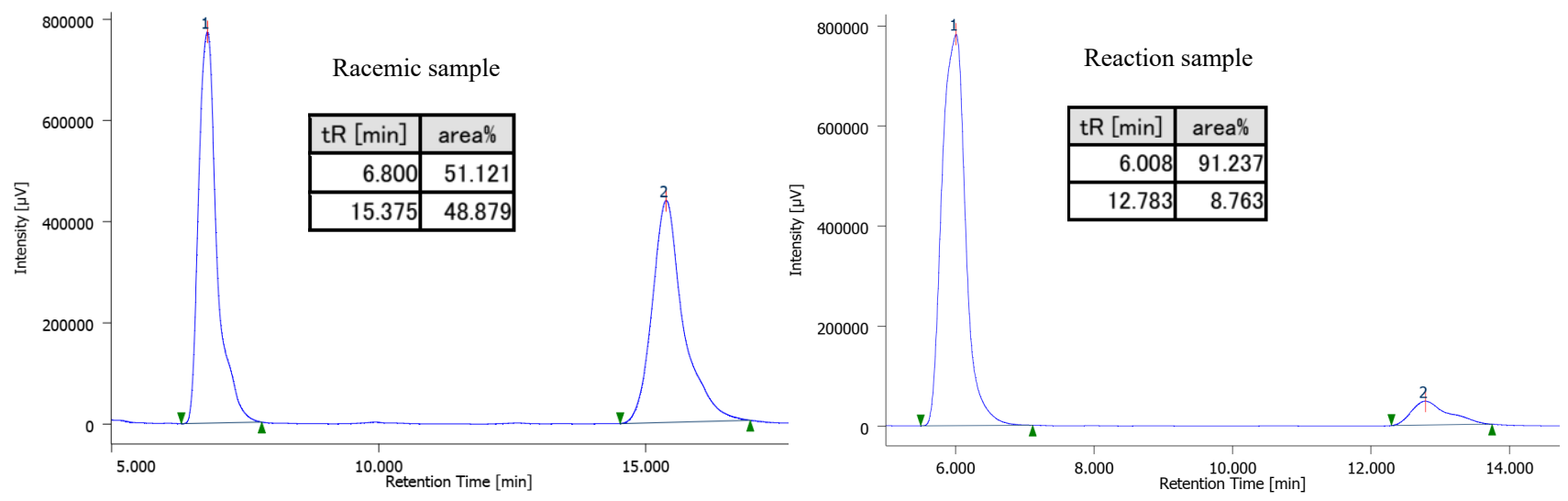

(S)-4-(Cyclohexylmethyl)-4-(2-methylbenzyl)isoxazolidin-5-one (7c-B)

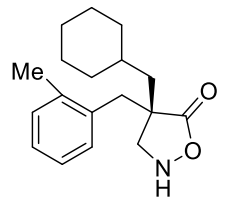

Prepared by the general procedure F from 7 c $(57.4 \mathrm{mg}, 0.2 \mathrm{mmol})$ and NMR yield was 53\% and isolated by PTLC (40\% hexane : EtOAc with $1 \% \mathrm{MeOH})$ as a white solid (28.1 $\mathrm{mg}, 49 \%$ yield); $[\alpha]_{\mathrm{D}}^{27}+42.18$ (c $0.9, \mathrm{CHCl}_{3}, 70 \%$ ee sample). Conversion $47 \%$; Selectivity factor $(s) 17$.

Enantiomeric excess of the product was determined to be $70 \%$ ee by chiral stationary phase HPLC analysis (CHIRALPAK IC $(\phi 0.46 \mathrm{~cm} \times 25 \mathrm{~cm}), n$-hexane $/ 2$-propanol $=80 / 20$, flow rate $0.8 \mathrm{~mL} / \mathrm{min}$, detection at $254 \mathrm{~nm}, \mathrm{t}_{R}=13.36 \min$ (major), 18.37 min (minor).
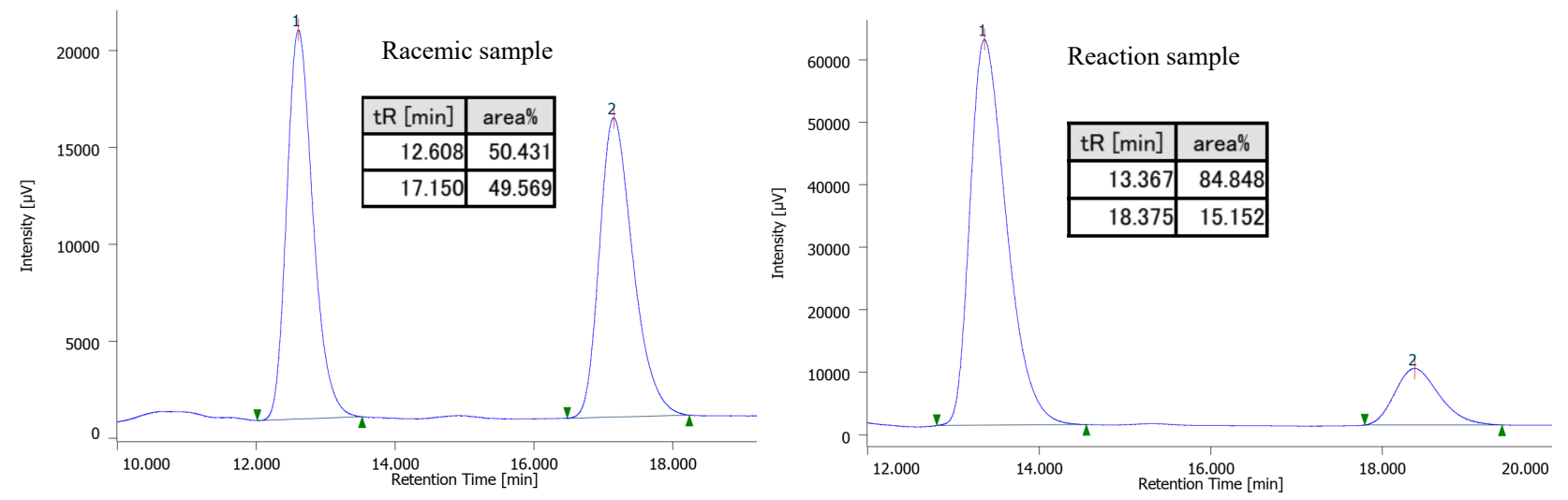

\section{Methyl (R)-3-(cyclohexylmethyl)-5-methyl-1,2,3,4-tetrahydroquinoline-3-carboxylate (9c-A methyl ester)}<smiles>CCNc1ccccc1CC1(C(=O)OC)CCCCC1</smiles>

Prepared by the general procedure F from 7c $(57.4 \mathrm{mg}, 0.2 \mathrm{mmol})$ and NMR yield of 9c-A was $30 \%$ and estrification compound was isolated by silica gel column chromatgraphy (hexane/ethyl acetate) as a white solid (16.2 mg, 27\% yield); m.p. $99-101{ }^{\circ} \mathrm{C} ;{ }^{1} \mathbf{H} \mathbf{N M R}\left(400 \mathrm{MHz}, \mathrm{CDCl}_{3}\right): \delta$ $6.88(\mathrm{t}, J=7.7 \mathrm{~Hz}, 1 \mathrm{H}), 6.54(\mathrm{~d}, J=7.4 \mathrm{~Hz}, 1 \mathrm{H}), 6.37(\mathrm{~d}, J=8.0 \mathrm{~Hz}, 1 \mathrm{H}), 3.79(\mathrm{br}, 1 \mathrm{H}), 3.66(\mathrm{~s}$, 
3H), 3.45 (dd, $J=11.3,1.4 \mathrm{~Hz}, 1 \mathrm{H}), 3.13$ (d, $J=11.3 \mathrm{~Hz}, 1 \mathrm{H}), 3.06$ (d, $J=16.5 \mathrm{~Hz}, 1 \mathrm{H}), 2.52$ (d, $J=16.6 \mathrm{~Hz}, 1 \mathrm{H})$, $2.20(\mathrm{~s}, 3 \mathrm{H}), 1.69-1.59(\mathrm{~m}, 6 \mathrm{H}), 1.51-1.39(\mathrm{~m}, 2 \mathrm{H}), 1.27-1.08(\mathrm{~m}, 3 \mathrm{H}), 0.96-0.85(\mathrm{~m}, 2 \mathrm{H}) ;{ }^{13} \mathbf{C}$ NMR $(101$ $\left.\mathrm{MHz}, \mathrm{CDCl}_{3}\right): \delta 176.62,143.62,137.32,126.23,119.44,118.51,112.28,51.81,48.12,44.12,43.82,34.43,34.18$, 33.77, 33.16, 26.35, 26.22, 19.46; IR (thin film): 3385, 2918, 2850, 1717, 1617, 1583, 1508, 1449, 1289, 1195 , 1081, 976, 889, 853, $788 \mathrm{~cm}^{-1}$; HRMS (ESI) $\mathrm{m} / z$ calc'd for $\mathrm{C}_{19} \mathrm{H}_{28} \mathrm{O}_{2} \mathrm{~N}[\mathrm{M}+\mathrm{H}]^{+}:$302.2115, found: 302.2113; $[\alpha]_{\mathrm{D}}{ }^{27}+65.48\left(c 0.6, \mathrm{CHCl}_{3}, 77 \%\right.$ ee sample). Enantiomeric excess of the product was determined to be $77 \%$ ee by chiral stationary phase HPLC analysis (CHIRALPAK IA $(\phi 0.46 \mathrm{~cm} \times 25 \mathrm{~cm}), n$-hexane/2-propanol $=90 / 10$, flow rate $1.0 \mathrm{~mL} / \mathrm{min}$, detection at $254 \mathrm{~nm}, \mathrm{t}_{R}=5.35 \mathrm{~min}$ (major), $11.22 \mathrm{~min}$ (minor).
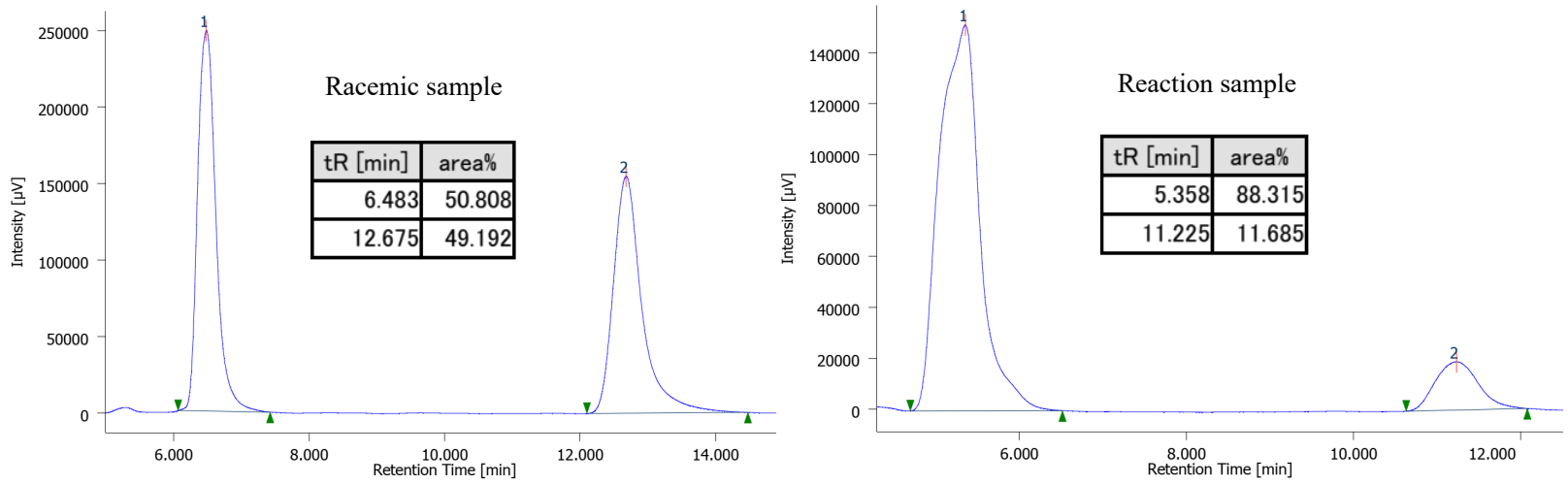

\section{(S)-4-([1,1'-Biphenyl]-4-ylmethyl)-4-(cyclohexylmethyl)isoxazolidin-5-one (7d-B)}

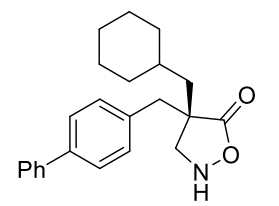

Prepared by the general procedure F from $7 \mathbf{d}(69.8 \mathrm{mg}, 0.2 \mathrm{mmol})$ and NMR yield was $44 \%$ and isolated by PTLC (40\% hexane : EtOAc with $1 \% \mathrm{MeOH})$ as a white solid (27.9 $\mathrm{mg}, 40 \%$ yield). $[\alpha]_{\mathrm{D}}^{27}+65.44$ (c 1.0, $\mathrm{CHCl}_{3}, 98.3 \%$ ee sample). Conversion $56 \%$; Selectivity factor $(s) 36$. Enantiomeric excess of the product was determined to be $98.3 \%$ ee by chiral stationary phase HPLC analysis (CHIRALPAK IA $(\phi 0.46 \mathrm{~cm} \times 25 \mathrm{~cm}$ ), $n$-hexane $/ 2$-propanol = 90/10, flow rate $1.0 \mathrm{~mL} / \mathrm{min}$, detection at $254 \mathrm{~nm}, \mathrm{t}_{R}=31.71 \mathrm{~min}$ (minor), $39.13 \mathrm{~min}$ (major).
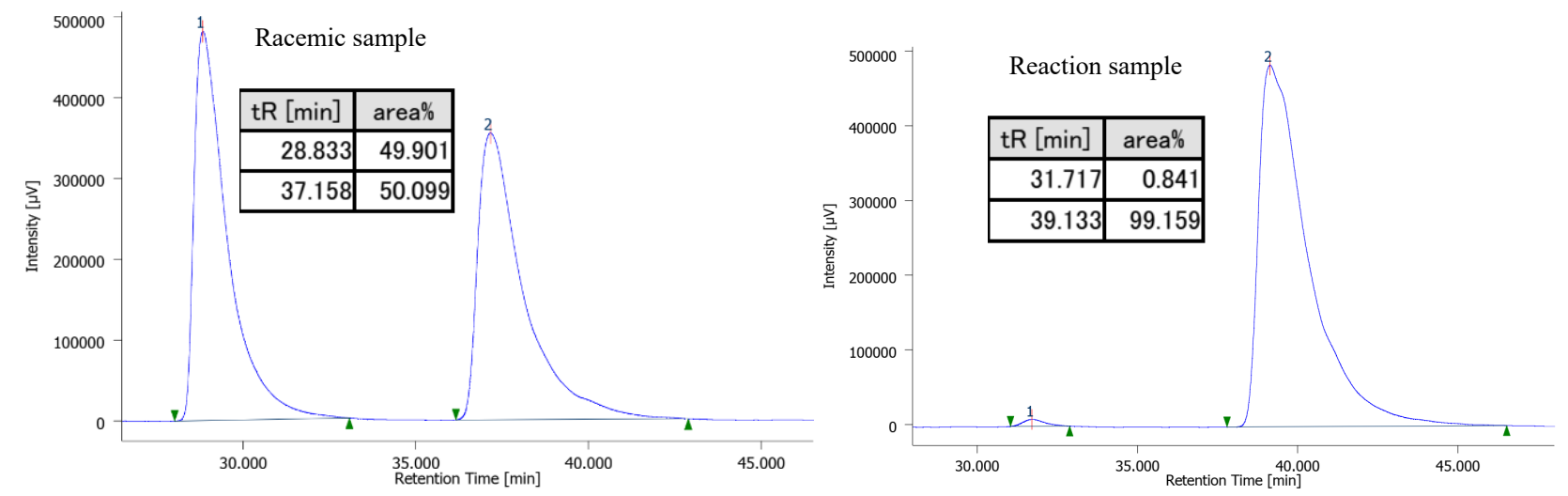


\section{Methyl (R)-3-(cyclohexylmethyl)-7-phenyl-1,2,3,4-tetrahydroquinoline-3-carboxylate (9d-A methyl ester)}

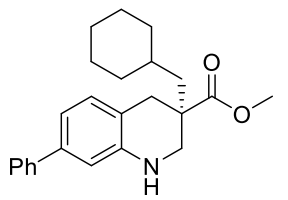

Prepared by the general procedure F from 7d $(69.8 \mathrm{mg}, 0.2 \mathrm{mmol})$ and NMR yield of 9d-A

was $43 \%$ and estrification compound was isolated by silica gel column chromatgraphy (hexane/ethyl acetate) as a white solid (28.3 mg, 39\% yield. m.p. $84-86{ }^{\circ} \mathrm{C} ;{ }^{1} \mathbf{H}$ NMR (400 $\left.\mathrm{MHz}, \mathrm{CDCl}_{3}\right): \delta 7.55-7.52(\mathrm{~m}, 2 \mathrm{H}), 7.40-7.37(\mathrm{~m}, 2 \mathrm{H}), 7.31-7.27(\mathrm{~m}, 1 \mathrm{H}), 7.06(\mathrm{~d}, J=$ $7.8 \mathrm{~Hz}, 1 \mathrm{H}), 6.88(\mathrm{dd}, J=7.8,1.8 \mathrm{~Hz}, 1 \mathrm{H}), 6.70(\mathrm{~d}, J=1.7 \mathrm{~Hz}, 1 \mathrm{H}), 3.93(\mathrm{br}, 1 \mathrm{H}), 3.67(\mathrm{~s}, 3 \mathrm{H}), 3.53(\mathrm{dd}, J=11.5$, $1.6 \mathrm{~Hz}, 1 \mathrm{H}), 3.24$ (d, $J=16.2 \mathrm{~Hz}, 1 \mathrm{H}), 3.19$ (d, $J=11.0 \mathrm{~Hz}, 1 \mathrm{H}), 2.73(\mathrm{~d}, J=16.2 \mathrm{~Hz}, 1 \mathrm{H}), 1.70-1.61(\mathrm{~m}, 6 \mathrm{H})$, $1.53-1.48(\mathrm{~m}, 2 \mathrm{H}), 1.26-1.09$ (m, 3H), $0.96-0.89$ (m, 2H); ${ }^{13} \mathbf{C}$ NMR (101 MHz, $\left.\mathrm{CDCl}_{3}\right): \delta$ 175.4, 142.7, 140.4, 138.9, 129.0, 127.5, 125.9, 125.9, 118.0, 115.7, 111.7, 50.8, 47.8, 42.8, 42.6, 34.4, 33.5, 33.1, 32.8, 25.3, 25.3, 25.2; IR (thin film): 3406, 2921, 2848, 1728, 1615, 1568, 1487, 1445, 1206, 1022, 861, $758 \mathrm{~cm}^{-1}$; HRMS (ESI) $\mathrm{m} / z$ calc'd for $\mathrm{C}_{24} \mathrm{H}_{30} \mathrm{O}_{2} \mathrm{~N}[\mathrm{M}+\mathrm{H}]^{+}: 364.2271$, found: 364.2267 ; $[\alpha]_{\mathrm{D}}{ }^{27}+38.94$ (c 1.0, $\mathrm{CHCl}_{3}, 59.6 \%$ ee sample). Enantiomeric excess of the product was determined to be $59.6 \%$ ee by chiral stationary phase HPLC analysis (CHIRALPAK IA-3 $(\phi 0.46 \mathrm{~cm} \times 25 \mathrm{~cm}), n$-hexane $/ 2$-propanol $=80 / 20$, flow rate $1.0 \mathrm{~mL} / \mathrm{min}$, detection at $254 \mathrm{~nm}$, $\mathrm{t}_{R}=7.59 \min$ (minor), $13.08 \mathrm{~min}$ (major).
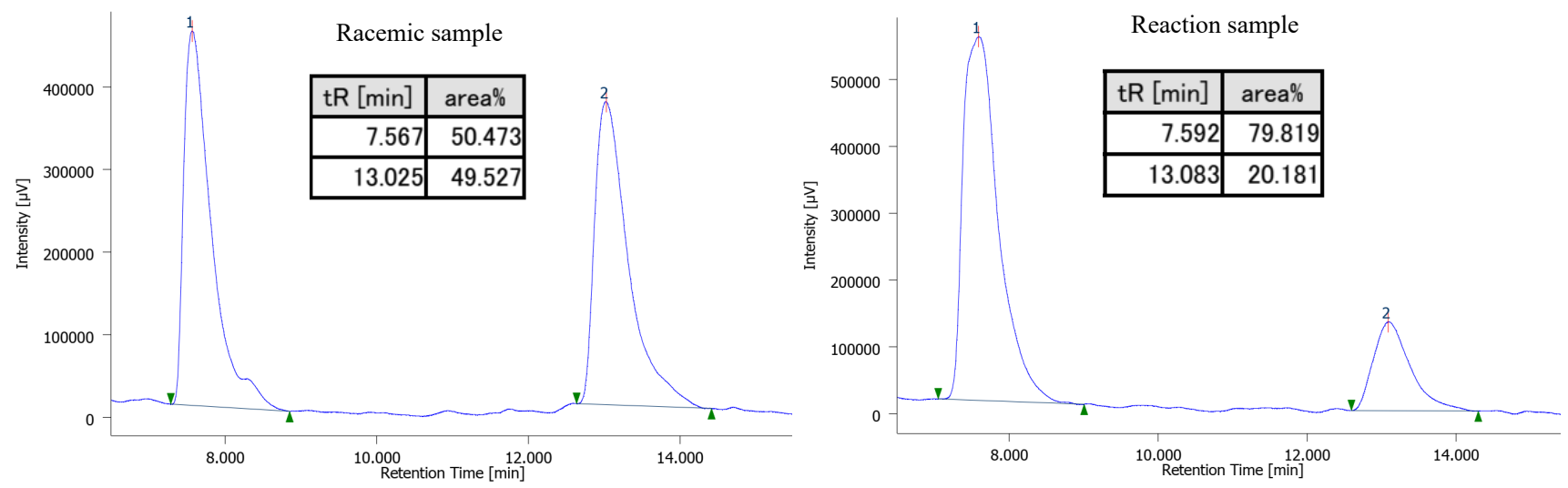

\section{(S)-4-Benzyl-4-((tetrahydro-2H-pyran-4-yl)methyl)isoxazolidin-5-one (7e-B)}

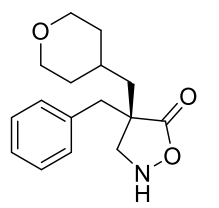

Prepared by the general procedure F from 7 e $(55.0 \mathrm{mg}, 0.2 \mathrm{mmol})$ and NMR yield was $25 \%$ and isolated by PTLC (40\% hexane : EtOAc with $1 \% \mathrm{MeOH})$ as a light orange liquid $(12.0 \mathrm{mg}, 22 \%$ yield). $[\alpha]_{\mathrm{D}}^{27}-10.40$ ( $c$ 1.0, $\mathrm{CHCl}_{3}, 100 \%$ ee sample). Conversion $75 \%$; Selectivity factor $(s) 52$.

Enantiomeric excess of the product was determined to be $>99 \%$ ee by chiral stationary phase HPLC analysis (CHIRALPAK IA $(\phi 0.46 \mathrm{~cm} \times 25 \mathrm{~cm}$ ), $n$-hexane $/ 2$-propanol = 80/20, flow rate $1.0 \mathrm{~mL} / \mathrm{min}$, detection at $254 \mathrm{~nm}, \mathrm{t}_{R}=16.14$ min (major). 

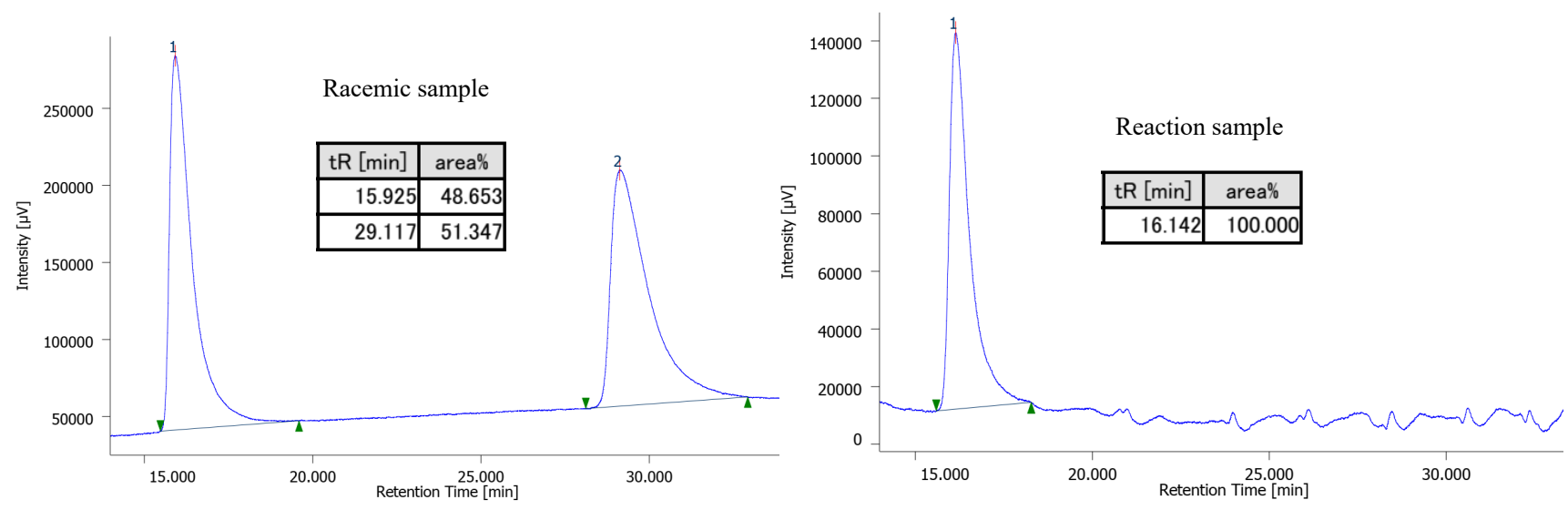

Methyl (R)-3-((tetrahydro-2H-pyran-4-yl)methyl)-1,2,3,4-tetrahydroquinoline-3-carboxylate (9e-A methyl

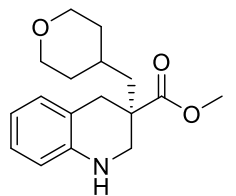
ester)

Prepared by the general procedure F from 7 e $(55.0 \mathrm{mg}, 0.2 \mathrm{mmol})$ and NMR yield of 9e-A was $69 \%$ and estrification compound was isolated by silica gel column chromatgraphy (hexane/ethyl acetate) as a colorless liquid $\left(35.8 \mathrm{mg}, 62 \%\right.$ yield. ${ }^{1} \mathbf{H}$ NMR (400 MHz, $\left.\mathrm{CDCl}_{3}\right): \delta 6.99-6.96(\mathrm{~m}, 2 \mathrm{H}), 6.65$

(td, $J=7.4,1.1 \mathrm{~Hz}, 1 \mathrm{H}), 6.49(\mathrm{dd}, J=8.4,1.1 \mathrm{~Hz}, 1 \mathrm{H}), 3.91-3.87$ (m, 2H), $3.83(\mathrm{br}, 1 \mathrm{H}), 3.66(\mathrm{~s}, 3 \mathrm{H}), 3.50$ (dd, $J$ $=11.5,1.5 \mathrm{~Hz}, 1 \mathrm{H}), 3.39-3.32(\mathrm{~m}, 2 \mathrm{H}), 3.22-3.16(\mathrm{~m}, 2 \mathrm{H}), 2.71(\mathrm{~d}, J=16.2 \mathrm{~Hz}, 1 \mathrm{H}), 1.69-1.64(\mathrm{~m}, 1 \mathrm{H}), 1.57$ - $1.48(\mathrm{~m}, 4 \mathrm{H}), 1.33-1.23(\mathrm{~m}, 2 \mathrm{H}) ;{ }^{13} \mathbf{C} \mathbf{~ N M R}\left(101 \mathrm{MHz}, \mathrm{CDCl}_{3}\right): \delta 176.2,143.3,129.7,126.9,119.4,117.8$, 114.2, 67.9, 51.9, 48.5, 43.4, 42.8, 35.7, 34.0, 33.7, 31.3; IR (thin film): 3390, 2922, 2838, 1725, 1606, 1499, 1437 , 1370, 1207, 1135, 981, 845, $745 \mathrm{~cm}^{-1}$; HRMS (ESI) $\mathrm{m} / z$ calc'd for $\mathrm{C}_{17} \mathrm{H}_{24} \mathrm{O}_{3} \mathrm{~N}[\mathrm{M}+\mathrm{H}]^{+}: 290.1751$, found: $290.1753 ;[\alpha]_{\mathrm{D}}{ }^{27}+33.72\left(c 0.8, \mathrm{CHCl}_{3}, 43 \%\right.$ ee sample). Enantiomeric excess of the product was determined to be $43 \%$ ee by chiral stationary phase HPLC analysis (CHIRALPAK IA-3 $(\phi 0.46 \mathrm{~cm} \times 25 \mathrm{~cm}), n$-hexane $/ 2$-propanol = $90 / 10$, flow rate $1.0 \mathrm{~mL} / \mathrm{min}$, detection at $254 \mathrm{~nm}, \mathrm{t}_{R}=7.25 \mathrm{~min}$ (major), $9.63 \mathrm{~min}$ (minor).
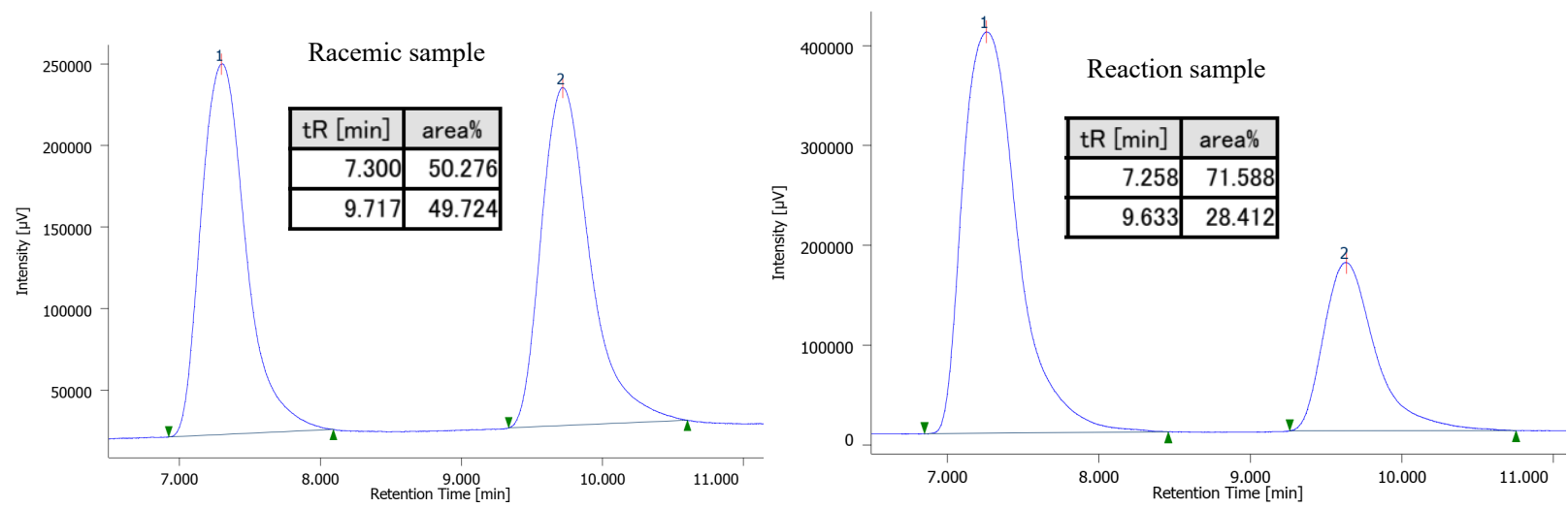


\section{(S)-4-Benzyl-4-(4-nitrobenzyl)isoxazolidin-5-one (7f-B)}

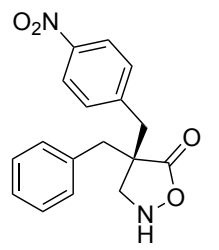

Prepared by the general procedure F from $7 f(62.4 \mathrm{mg}, 0.2 \mathrm{mmol})$ and NMR yield was $20 \%$ and isolated by PTLC (40\% hexane : EtOAc with $1 \% \mathrm{MeOH})$ as a white solid (10.6 $\mathrm{mg}, 17 \%$ yield). $[\alpha]_{\mathrm{D}}^{27}-6.00$ (c 1.0, acetone, $95.5 \%$ ee sample). Conversion $80 \%$; Selectivity factor $(s) 5$. Enantiomeric excess of the product was determined to be $95.5 \%$ ee by chiral stationary phase HPLC analysis (CHIRALPAK IB ( $\phi 0.46 \mathrm{~cm} \times 25 \mathrm{~cm}$ ), $n$-hexane/2-propanol = 90/10, flow rate $1.0 \mathrm{~mL} / \mathrm{min}$, detection at $254 \mathrm{~nm}, \mathrm{t}_{R}=48.25 \min$ (major), $60.11 \mathrm{~min}$ (minor).
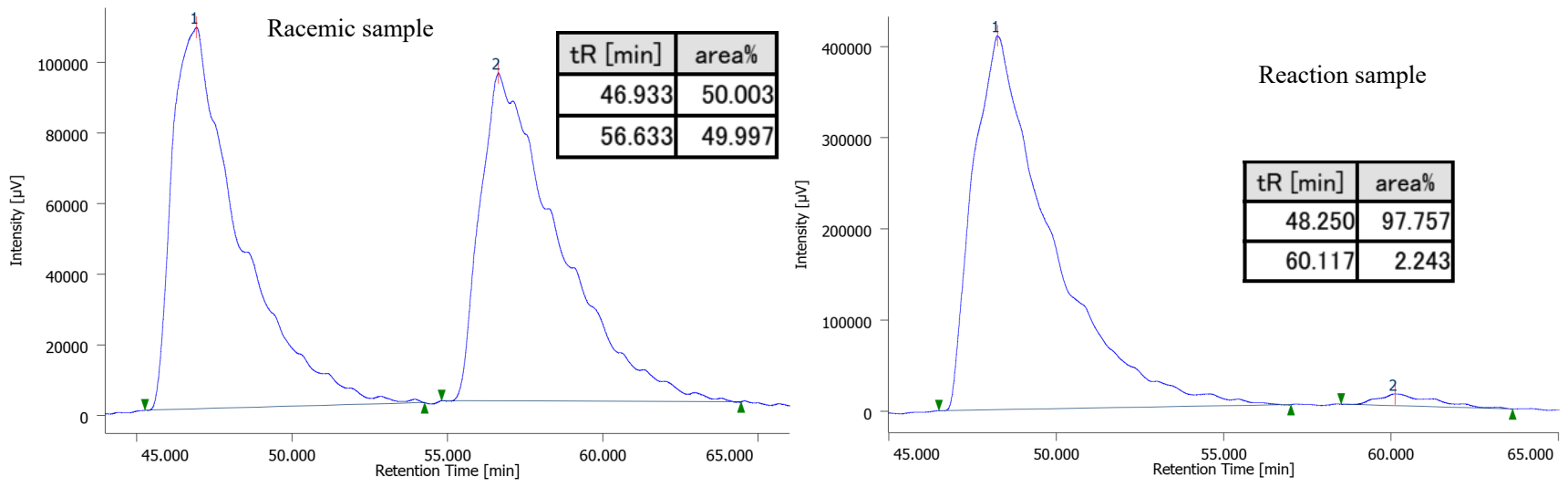

\section{Methyl (R)-3-(4-nitrobenzyl)-1,2,3,4-tetrahydroquinoline-3-carboxylate (9f-A methyl ester)}

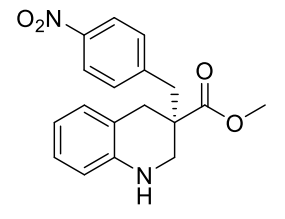

Prepared by the general procedure F from $7 \mathbf{f}(62.4 \mathrm{mg}, 0.2 \mathrm{mmol})$ and NMR yield of 9f-A was $80 \%$ and estrification compound was isolated by silica gel column chromatgraphy (hexane/ethyl acetate) as a light orange solid (48.0 mg, 74\% yield). m.p. $149-151{ }^{\circ} \mathrm{C} ;{ }^{1} \mathbf{H}$ NMR $(400 \mathrm{MHz}$, $\left.\mathrm{CDCl}_{3}\right): \delta 8.14-8.11(\mathrm{~m}, 2 \mathrm{H}), 7.28-7.27(\mathrm{~m}, 1 \mathrm{H}), 7.05-7.01(\mathrm{~m}, 1 \mathrm{H}), 6.99-6.98(\mathrm{~m}, 1 \mathrm{H})$, $6.69(\mathrm{td}, J=7.4,1.1 \mathrm{~Hz}, 1 \mathrm{H}), 6.57(\mathrm{dd}, J=8.0,0.8 \mathrm{~Hz}, 1 \mathrm{H}), 3.94(\mathrm{br}, 1 \mathrm{H}), 3.66(\mathrm{~s}, 3 \mathrm{H}), 3.46(\mathrm{~d}, J=11.7 \mathrm{~Hz}, 1 \mathrm{H})$, $3.21(\mathrm{dd}, J=11.7,1.9 \mathrm{~Hz}, 1 \mathrm{H}), 3.12(\mathrm{dd}, J=14.8,5.5 \mathrm{~Hz}, 2 \mathrm{H}), 3.02(\mathrm{~d}, J=13.3 \mathrm{~Hz}, 1 \mathrm{H}), 2.80(\mathrm{dd}, J=16.3,1.5$ $\mathrm{Hz}, 1 \mathrm{H}) ;{ }^{13} \mathbf{C}$ NMR (101 MHz, $\left.\mathrm{CDCl}_{3}\right): \delta 174.6,147.0,145.1,142.9,130.7,130.0,127.3,123.4,118.3,118.0$, 114.2, 52.0, 46.5, 45.6, 40.2, 35.0; IR (thin film): 3379, 2954, 2850, 1718, 1602, 1514, 1439, 1344, 1290, 1200, 1106, 970, 856, $746 \mathrm{~cm}^{-1}$; HRMS (ESI) $m / z$ calc'd for $\mathrm{C}_{18} \mathrm{H}_{19} \mathrm{O}_{4} \mathrm{~N}_{2}[\mathrm{M}+\mathrm{H}]^{+}: 327.1339$, found: 327.1342; $[\alpha]_{\mathrm{D}}{ }^{27}$ +7.02 (c 1.0, $\mathrm{CHCl}_{3}, 33.5 \%$ ee sample). Enantiomeric excess of the product was determined to be $33.5 \%$ ee by chiral stationary phase HPLC analysis (CHIRALPAK IA $(\phi 0.46 \mathrm{~cm}$ x $25 \mathrm{~cm}), n$-hexane/2-propanol =90/10, flow rate $1.0 \mathrm{~mL} / \mathrm{min}$, detection at $254 \mathrm{~nm}, \mathrm{t}_{R}=24.14 \mathrm{~min}$ (major), $31.21 \mathrm{~min}$ (minor). 

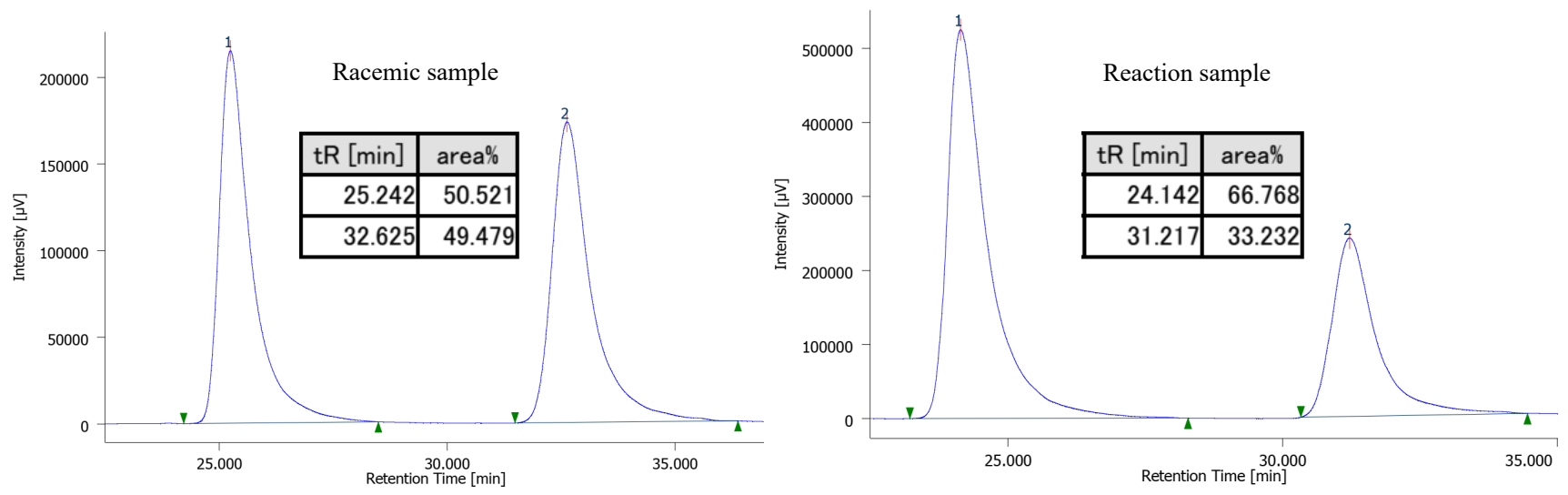

\section{(S)-4-Benzyl-4-methylisoxazolidin-5-one (7g-B)}

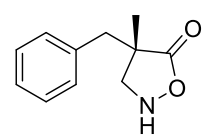

Prepared by the general procedure F from $7 \mathrm{~g}(38.2 \mathrm{mg}, 0.2 \mathrm{mmol})$ and NMR yield was $31 \%$ and isolated by PTLC (40\% hexane : EtOAc with 1\% MeOH) as a white solid (11.8 $\mathrm{mg}, 27 \%$ yield). $[\alpha]_{D}^{27}+12.34\left(c 0.5, \mathrm{CHCl}_{3}, 47.1 \%\right.$ ee sample). Conversion $69 \%$; Selectivity factor $(s) 2.3$; Enantiomeric excess of the product was determined to be $47.1 \%$ ee by chiral stationary phase HPLC analysis (CHIRALPAK ID ( $\phi 0.46 \mathrm{~cm} \times 25 \mathrm{~cm}), n$-hexane $/ 2$-propanol $=90 / 10$, flow rate $1.0 \mathrm{~mL} / \mathrm{min}$, detection at $254 \mathrm{~nm}$, $\mathrm{t}_{R}=15.59 \min$ (minor), $16.83 \min$ (major).
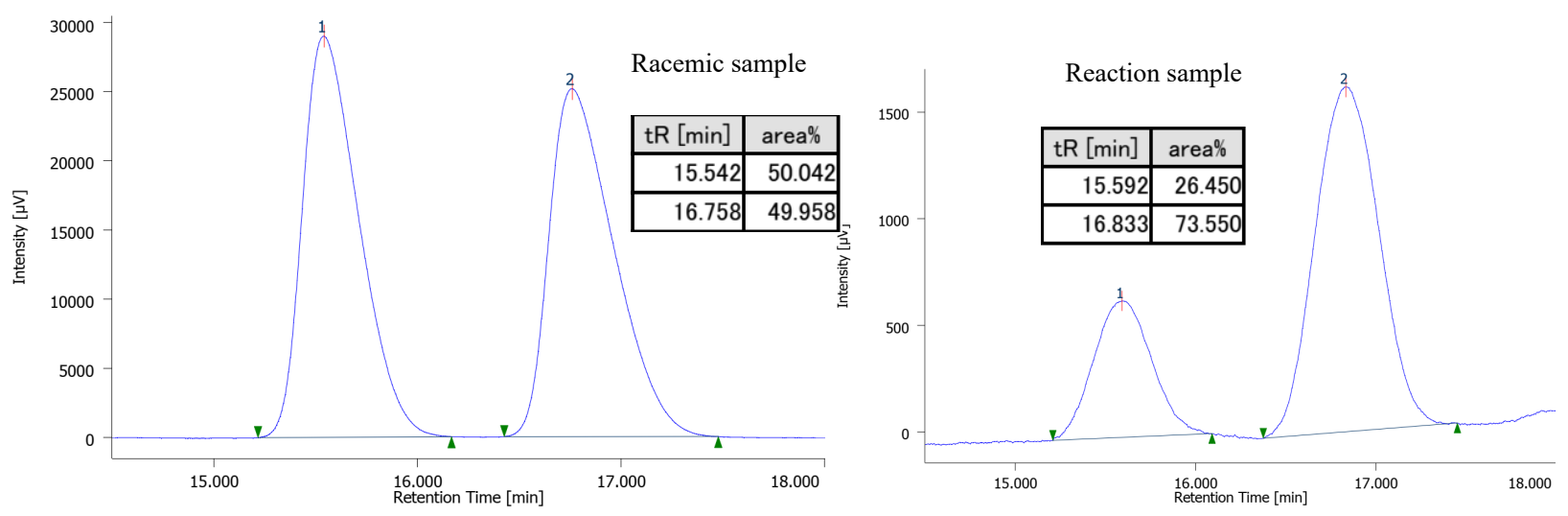

\section{Methyl (R)-3-methyl-1,2,3,4-tetrahydroquinoline-3-carboxylate (9g-A methyl ester)}

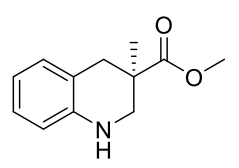

Prepared by the general procedure F from $7 \mathbf{g}(38.2 \mathrm{mg}, 0.2 \mathrm{mmol})$ and NMR yield of $\mathbf{9 g}-\mathbf{A}$ was $60 \%$ and estrification compound was isolated by silica gel column chromatgraphy (hexane/ethyl acetate) as a white solid (23.3 mg, 57\% yield). ${ }^{1} \mathbf{H}$ NMR (400 MHz, $\left.\mathrm{CDCl}_{3}\right): \delta 6.99-6.96(\mathrm{~m}$, 2H), $6.64(\mathrm{td}, J=7.4,1.1 \mathrm{~Hz}, 1 \mathrm{H}), 6.50(\mathrm{dd}, J=8.3,1.1 \mathrm{~Hz}, 1 \mathrm{H}), 3.89(\mathrm{br}, 1 \mathrm{H}), 3.68(\mathrm{~s}, 3 \mathrm{H}), 3.51(\mathrm{dd}, J=11.5,1.5$ $\mathrm{Hz}, 1 \mathrm{H}), 3.19(\mathrm{~d}, J=16.1 \mathrm{~Hz}, 1 \mathrm{H}), 3.12(\mathrm{dd}, J=11.5,1.5 \mathrm{~Hz}, 1 \mathrm{H}), 2.67(\mathrm{~d}, J=16.1 \mathrm{~Hz}, 1 \mathrm{H}), 1.27(\mathrm{~s}, 3 \mathrm{H}) ;{ }^{13} \mathrm{C}$ NMR (101 MHz, $\left.\mathrm{CDCl}_{3}\right): \delta$ 176.60, 142.99, 129.75, 126.92, 119.41, 117.56, 114.13, 52.09, 49.11, 40.40, 36.50, 22.30; IR (thin film): 3410, 2951, 2932, 2843, 1729, 1608, 1500, 1454, 1324, 1285, 1229, 1114, 1077, 990, 748 $\mathrm{cm}^{-1}$; HRMS (ESI) $\mathrm{m} / z$ calc'd for $\mathrm{C}_{12} \mathrm{H}_{16} \mathrm{O}_{2} \mathrm{~N}\left[\mathrm{M}+\mathrm{H}^{+}: 206.1176\right.$, found: 206.1177 ; $[\alpha]_{\mathrm{D}}{ }^{27}+35.29\left(c 1.4, \mathrm{CHCl}_{3}\right.$, 
$42 \%$ ee sample). Enantiomeric excess of the product was determined to be $42 \%$ ee by chiral stationary phase HPLC analysis (CHIRALPAK IA $(\phi 0.46 \mathrm{~cm} \times 25 \mathrm{~cm}), n$-hexane/2-propanol $=90 / 10$, flow rate $1.0 \mathrm{~mL} / \mathrm{min}$, detection at $254 \mathrm{~nm}, \mathrm{t}_{R}=8.85 \mathrm{~min}$ (major), $10.88 \mathrm{~min}$ (minor).
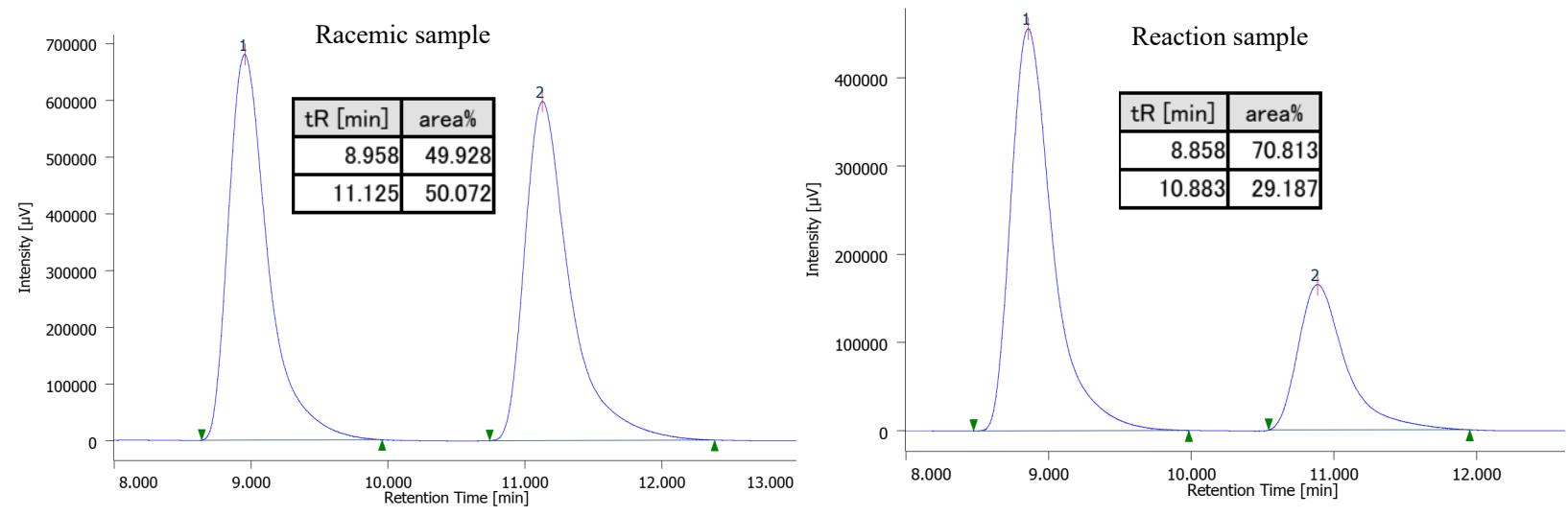


\section{Cu/BOX-catalyzed electrophilic amination}

\section{5-1. Limitations of $\mathrm{Cu} / \mathrm{BOX}$-catalyzed asymmetric desymmetrization}

Table S1. Limitations of previous catalytic conditions.

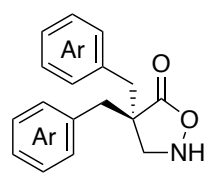

1) $\mathrm{CuOTf} \cdot 0.5 \mathrm{C}_{6} \mathrm{H}_{6}(5 \mathrm{~mol} \%)$

(S)-tBu-BOX (10 mol\%)

HFIP, $0^{\circ} \mathrm{C}$, time

2) $\mathrm{TMSCHN}_{2}$

9:1 toluene/MeOH

\section{Product}

2a

$\mathrm{C}_{6} \mathrm{H}_{5}$

$2 \mathbf{b}$

2c

2f

$2 \mathrm{~g}$
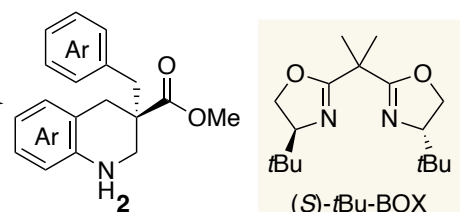

(S)-tBu-BOX ee $(\%)$

93

70

ND

80

ND

Discussion: As shown in Table S1, a slight deviation from 1a led to unsatisfactory results. Introduction of a methyl group at the para position reduced the reactivity and selectivity (entry 2). In addition, introducing the same substituent at the ortho position almost inhibited the desired cyclization, and most of the substrate remained unchanged (entry 3). Introduction of an electron-withdrawing group further deteriorated the situation. The substrate bearing a bromine atom at the para position afforded the product with a reasonable selectivity, albeit in low yield (entry 4). The ortho-chloro substrate provided a negligible quantity of product $\mathbf{2 g}$ (entry 5). In contrast to methylsubstituted cases, both halogen-substituted substrates yielded significant amounts of unidentified products.

\section{5-2. Unexpected product formation under the $\mathrm{Cu} / \mathrm{BOX}$ catalytic conditions}

Scheme S1. Cu/Box-catalyzed electrophilic amination of S11.

a)

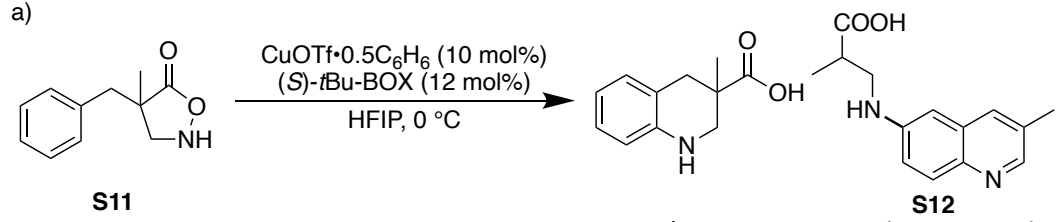

b)

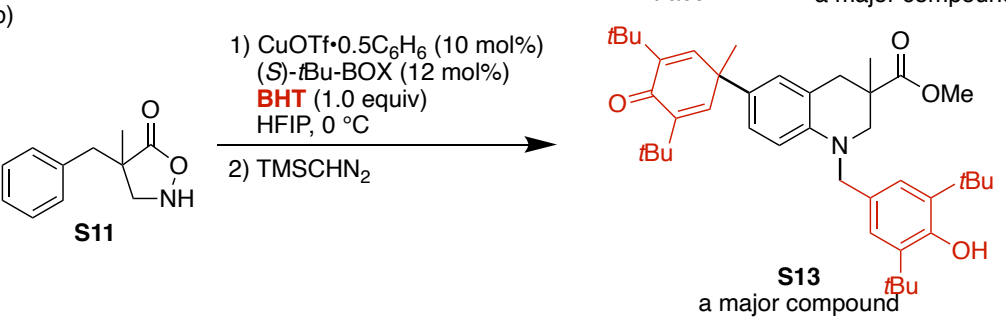

Discussion: With $\alpha$-Me substrate S11, rather unexpected observations were made. The $\mathrm{Cu} / \mathrm{BOX}$ catalytic system gave only a trance amount of the desired product. Instead, several compounds were formed under the conditions, one of which was isolated and characterized as dimeric compound S12. The methyl quinoline substructure indicates that the desired cyclization likely occurred, albeit accompanied by subsequent decomposition reactions, including aromatization and decarboxylation. Although we believe that the $\mathrm{C}-\mathrm{N}$ bond forming cyclization proceeds via a copper-bound alkyl nitrene rather than a naked nitrogen-centered radical, the bond between the monomer 
units in S12 is also indicative of the involvement of such N-radical. Furthermore, the addition of 1 equiv BHT led to the isolation of $\mathbf{S 1 3}$. The structure of $\mathbf{S 1 3}$ clearly shows that a radical was generated before the decarboxylation and after the six-membered ring cyclization. The different connectivity patterns for the two BHT moieties illustrate that $\mathrm{BHT}-\mathrm{C}(\mathrm{sp} 2)$ bond was formed via an open-shell radical pathway, whereas the BHT-N bond was formed via a closed-shell 1,6-addition pathway.

\section{Methyl 2-benzyl-2-methyl-3-((3-methylquinolin-6-yl)amino)propanoate (methyl ester of S12)}

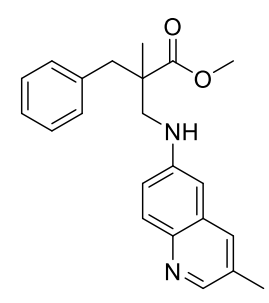

By a slight modification of the general procedure F, the compound was formed from S11 (19.1 $\mathrm{mg}$ ) and isolated by PTLC (hexane/ethyl acetate) as a pale green solid (5.2 $\mathrm{mg}, 15 \%$ yield). m.p. 92-94 ${ }^{\circ} \mathrm{C} ;{ }^{1} \mathbf{H}$ NMR $\left(600 \mathrm{MHz}, \mathrm{CD}_{3} \mathrm{OD}\right) \delta 8.31(\mathrm{~d}, J=1.5 \mathrm{~Hz}, 1 \mathrm{H}), 7.81(\mathrm{~s}, 1 \mathrm{H}), 7.68(\mathrm{~d}, J=$ $9.1 \mathrm{~Hz}, 1 \mathrm{H}), 7.25-7.18(\mathrm{~m}, 4 \mathrm{H}), 7.14-7.13(\mathrm{~m}, 2 \mathrm{H}), 6.73(\mathrm{~d}, J=2.5 \mathrm{~Hz}, 1 \mathrm{H}), 3.62(\mathrm{~s}, 3 \mathrm{H})$,

$2.44(\mathrm{~s}, 3 \mathrm{H}), 1.24(\mathrm{~s}, 3 \mathrm{H}) ;{ }^{13} \mathrm{C}$ NMR (151 MHz, CD $\left.3 \mathrm{OD}\right) \delta 177.8,148.9,147.7,141.5,138.4,134.8,132.2,131.8$, 131.2, 129.2, 129.1, 127.7, 122.5, 103.1, 52.3, 51.7, 50.0, 43.8, 20.6, 18.6; IR (thin film): 3348, 3027, 2948, 1725, 1624, 1517, 1496, 1433, 1251, 1119, 883, 821, $741 \mathrm{~cm}^{-1}$; HRMS (ESI) $\mathrm{m} / z$ calc'd for $\mathrm{C}_{22} \mathrm{H}_{25} \mathrm{O}_{2} \mathrm{~N}_{2}[\mathrm{M}+\mathrm{H}]^{+}$: 349.1911, found: 349.1916.

\section{Methyl 6-(3,5-di-tert-butyl-1-methyl-4-oxocyclohexa-2,5-dien-1-yl)-1-(3,5-di-tert-butyl-4-hydroxybenzyl)-3-} methyl-1,2,3,4-tetrahydroquinoline-3-carboxylate (S13)

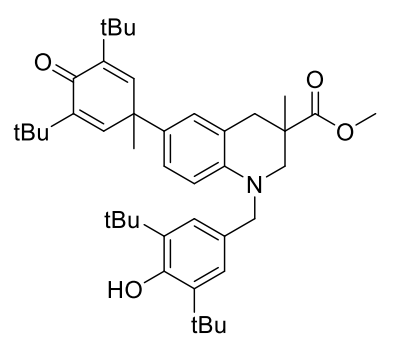

By a slight modification of the general procedure F, the compound was formed from S11 $(19.1 \mathrm{mg})$ and isolated by PTLC (hexane/ethyl acetate) as a yellow solid (7.1 $\mathrm{mg}, 11 \%$ yield). m.p. $68-70{ }^{\circ} \mathrm{C} ;{ }^{1} \mathbf{H}$ NMR $\left(400 \mathrm{MHz}, \mathrm{CDCl}_{3}\right) \delta 7.02(\mathrm{~s}, 2 \mathrm{H}), 6.88-6.83(\mathrm{~m}, 2 \mathrm{H})$, $6.58-6.54(\mathrm{~m}, 2 \mathrm{H}), 5.09(\mathrm{~s}, 1 \mathrm{H}), 4.45(\mathrm{~d}, J=16.3 \mathrm{~Hz}, 1 \mathrm{H}), 4.29(\mathrm{~d}, J=16.3 \mathrm{~Hz}, 1 \mathrm{H})$, $3.66(\mathrm{~s}, 3 \mathrm{H}), 3.49(\mathrm{~d}, J=11.4 \mathrm{~Hz}, 1 \mathrm{H}), 3.21-3.15(\mathrm{~m}, 2 \mathrm{H}), 2.68(\mathrm{~d}, J=15.9 \mathrm{~Hz}, 1 \mathrm{H})$, $1.54(\mathrm{~s}, 3 \mathrm{H}), 1.39(\mathrm{~s}, 18 \mathrm{H}), 1.29(\mathrm{~s}, 3 \mathrm{H}), 1.22(\mathrm{~s}, 18 \mathrm{H}) ;{ }^{13} \mathbf{C} \mathbf{N M R}\left(101 \mathrm{MHz}, \mathrm{CDCl}_{3}\right) \delta$ $186.7,176.4,152.7,147.7,147.7,143.9,143.9,143.6,136.0,129.2,128.8,127.2,125.2,123.5,120.1,111.8,56.3$, 55.6, 52.1, 42.7, 40.7, 37.4, 34.6, 34.3, 30.3, 29.5, 24.9, 22.2; IR (thin film): 3405, 2954, 2870, 1732, 1654, 1509, 1434, 1361, 1243, 1105, 1026, 878, 808, $741 \mathrm{~cm}^{-1}$; HRMS (ESI) $\mathrm{m} / z$ calc'd for $\mathrm{C}_{42} \mathrm{H}_{60} \mathrm{O}_{4} \mathrm{~N}[\mathrm{M}+\mathrm{H}]^{+}: 642.4509$, found: 642.4506 . 


\section{Synthetic application}

\section{6-1. Large scale catalytic asymmetric desymmetrization}
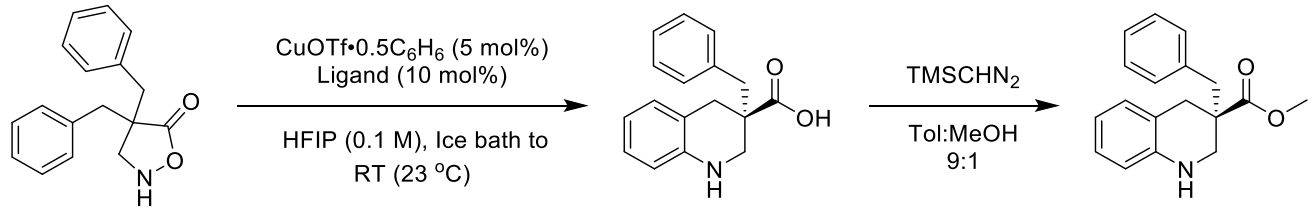

To a flame-dried $100 \mathrm{~mL}$ test tube equipped with a magnetic stirring bar were added $\mathbf{L 1}$ (107 $\mathrm{mg}, 0.45 \mathrm{mmol}, 12$ $\mathrm{mol} \%$ ) and $\mathrm{CuBr}(53.5 \mathrm{mg}, 0.37 \mathrm{mmol}, 10 \mathrm{~mol} \%)$ in glow box, followed by the addition of HFIP (28 mL) and the mixture was stirred for 15 minutes at an ambient temperature. After that, the reaction was cooled with an ice bath, and substrate 1a $(1.0 \mathrm{~g}, 3.74 \mathrm{mmol})$ dissolved in $10 \mathrm{~mL}$ HFIP was added as a solution. The reaction mixture was stirred for $1 \mathrm{~h}$ and was quenched by the addition of aq EDTA and water. The aqueous layer was extracted with EtOAc $(3 \mathrm{x})$. The combined organic layers were concentrated under reduced pressure. The obtained residue was dissolved in 9:1 toluene: $\mathrm{MeOH}(40 \mathrm{~mL}, 0.1 \mathrm{M})$. To the resulting solution cooled with an ice bath was added (trimethylsilyl)diazomethane (2.0 M in $\mathrm{Et}_{2} \mathrm{O}, 2.8 \mathrm{~mL}, 1.5$ equiv). After $15 \mathrm{~min}$, the reaction was quenched by the addition of aq $\mathrm{AcOH}$, followed by sat aq $\mathrm{NaHCO}_{3}$. The aqueous layer was extracted with EtOAc (3x). The combined organic layers were concentrated under reduced pressure to give the crude product, which was purified by silica gel column chromatography (hexane/ethyl acetate) to give $\mathbf{2 a}$ as a white solid (985 $\mathrm{mg}, 94 \%$ yield). Ee of the product was determined to be $92 \%$ ee by chiral stationary phase HPLC analysis (CHIRALPAK IA $(\phi 0.46 \mathrm{~cm} \mathrm{x}$ $25 \mathrm{~cm}$ ), $n$-hexane $/ 2$-propanol $=80 / 20$, flow rate $1.0 \mathrm{~mL} / \mathrm{min}$, detection at $254 \mathrm{~nm}, \mathrm{t}_{R}=6.85 \min$ (major), $11.97 \mathrm{~min}$ (minor).
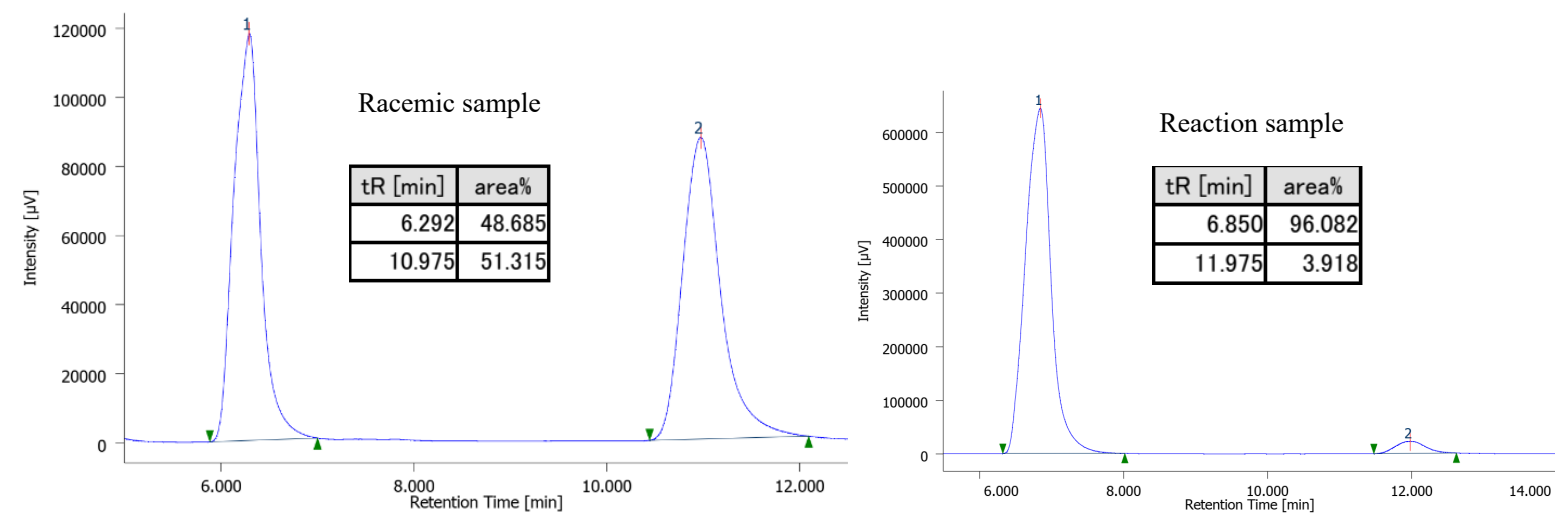

\section{6-2. Kinetic resolution of $7 \mathrm{a}$ and its further transformation}

\section{(S)-4-Benzyl-4-(cyclohexylmethyl)isoxazolidin-5-one (7a-B)}

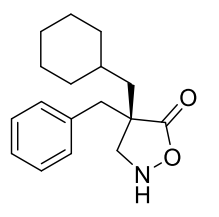

Prepared by the general procedure F from $7 \mathbf{a}(54.6 \mathrm{mg}, 0.2 \mathrm{mmol})$, sittered for $5 \mathrm{~h}$, and NMR yield was $27 \%$ and isolated by PTLC (40\% hexane : EtOAc with $1 \% \mathrm{MeOH})$ as a white solid (12.5 mg, $23 \%$ yield). Conversion 73\%; Selectivity factor $(s) 58$; Enantiomeric excess of the product was determined to be $>99 \%$ ee by chiral stationary phase HPLC analysis (CHIRALPAK IA $(\phi 0.46 \mathrm{~cm}$ 
x $25 \mathrm{~cm}$ ), $n$-hexane/2-propanol $=80 / 20$, flow rate $0.8 \mathrm{~mL} / \mathrm{min}$, detection at $254 \mathrm{~nm}, \mathrm{t}_{R}=10.68$ min (major).
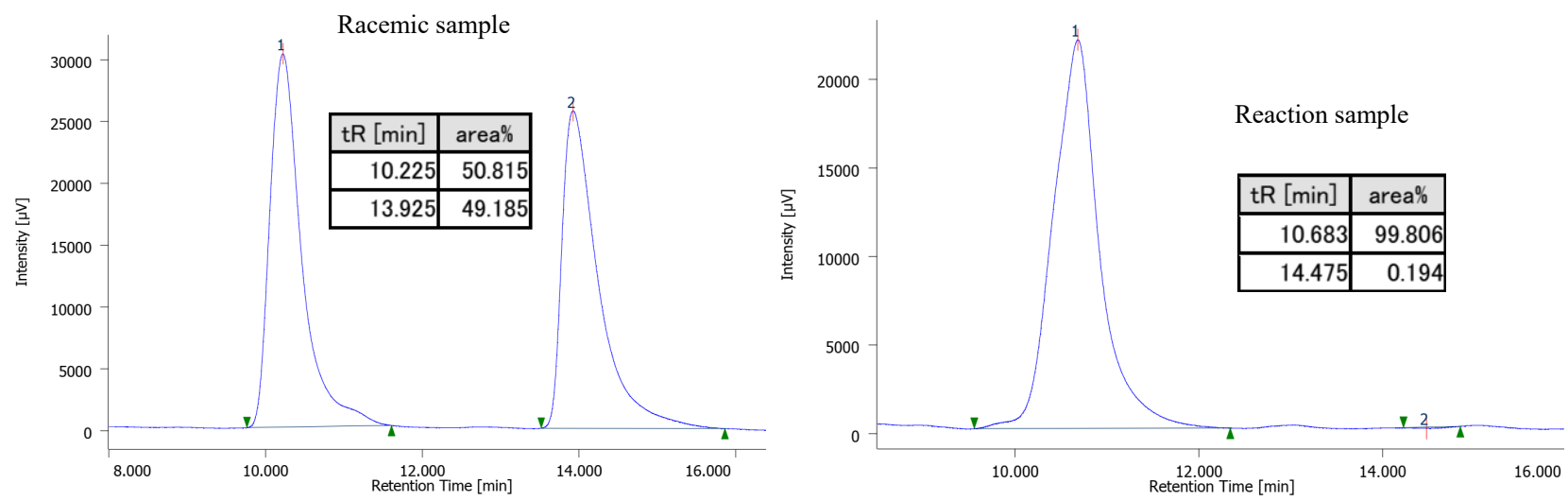

\section{(S)-3-Amino-2-benzyl-2-(cyclohexylmethyl)propanoic acid (10)}

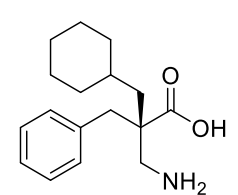

The title compound was prepared by following the reported procedure. ${ }^{5}$ To a solution of $7 \mathbf{a}-\mathbf{B}$ $(12.5 \mathrm{mg},>99 \%$ ee sample, $0.045 \mathrm{mmol})$ in $\operatorname{THF}(0.5 \mathrm{~mL})$ were added acetic acid $(1.0 \mathrm{~mL})$ and $\mathrm{H}_{2} \mathrm{O}(1.0 \mathrm{~mL})$, followed by the addition of $\mathrm{Zn}$ powder (118 g, 40 equiv). After being stirred for 16 $\mathrm{h}$ at room temperature, $\mathrm{H}_{2} \mathrm{O}$ was added to the reaction mixture, and the aqueous phase was extracted with EtOAc (3x). The combined organic layers were washed with brine, dried over $\mathrm{Na}_{2} \mathrm{SO}_{4}$, filtered, and concentrated under reduced pressure to give $\mathbf{1 0}$ as an amorphous $(11 \mathrm{mg}, 88 \%$ yield) in an analytically pure form. m.p. $195-197{ }^{\circ} \mathrm{C}$ (decomp.); ${ }^{1} \mathbf{H}$ NMR (400 MHz, CD $\left.{ }_{3} \mathrm{OD}\right) 7.28-7.18$ (m, 5H), 3.14 (d, $\left.J=13.8 \mathrm{~Hz}, 1 \mathrm{H}\right), 2.82$ (dt, $J=20.8,12.9 \mathrm{~Hz}, 3 \mathrm{H}), 1.82-1.48(\mathrm{~m}, 7 \mathrm{H}), 1.36-1.23(\mathrm{~m}, 3 \mathrm{H}), 1.19-0.88(\mathrm{~m}, 3 \mathrm{H}) ;{ }^{13} \mathbf{C} \mathbf{N M R}(101 \mathrm{MHz}$, $\left.\mathrm{CD}_{3} \mathrm{OD}\right) \delta 180.3,137.5,130.1,127.9,126.2,44.2,42.8,40.9,34.8,34.7,33.6,26.2,26.1,26.0$; IR (thin film): 2923, 2850, 2129, 1567, 1495, 1448, 1398, 1336, 1138, 997, 842, $701 \mathrm{~cm}^{-1}$; HRMS (ESI) $\mathrm{m} / z$ calc'd for $\mathrm{C}_{17} \mathrm{H}_{26} \mathrm{O}_{2} \mathrm{~N}[\mathrm{M}+\mathrm{H}]^{+}: 276.1958$, found: $276.1958 ;[\alpha]_{\mathrm{D}}{ }^{27}-2.70(c 1.0, \mathrm{MeOH})$. 


\section{References}

(1) Fulmer, G. R.; Miller, A. J. M.; Sherden, N. H.; Gottlieb, H. E.; Nudelman, A.; Stoltz, B. M.; Bercaw, J. E.; Goldberg, K. I. Organometallics, 2010, 29, 2176.

(2) Binder J. T., Cordier C. J., Fu G. C., J. Am. Chem. Soc. 2012, 134,17003-17006.

(3) (a) Yu, J.-S.; Espinosa, M.; Noda, H.; Shibasaki, M. J. Am. Chem. Soc. 2019, 141, 10530-10537. (b) Espinosa, M.; Noda, H.; Shibasaki, M. Org. Lett. 2019, 21, 9296-9299 (c) Tak, R. K., Amemiya, F., Noda, H., Shibasaki, M. Chem. Sci. 2021, 12, 7809-7817.

(4) Burés, J. Angew. Chem. Int. Ed. 2016, 55, 2028-2031.

(5) Yu, J.-S.; Noda, H.; Shibasaki, M. Angew. Chem., Int. Ed. 2018, 57, 818-822. 


\section{NMR spectra}

(S)-4-(tert-Butyl)-5,5-diphenyl-2-(pyridin-2-yl)-4,5-dihydrooxazole (SL1)

${ }^{1} \mathrm{H}$ NMR (400 MHz, $\mathrm{CDCl}_{3}$ )
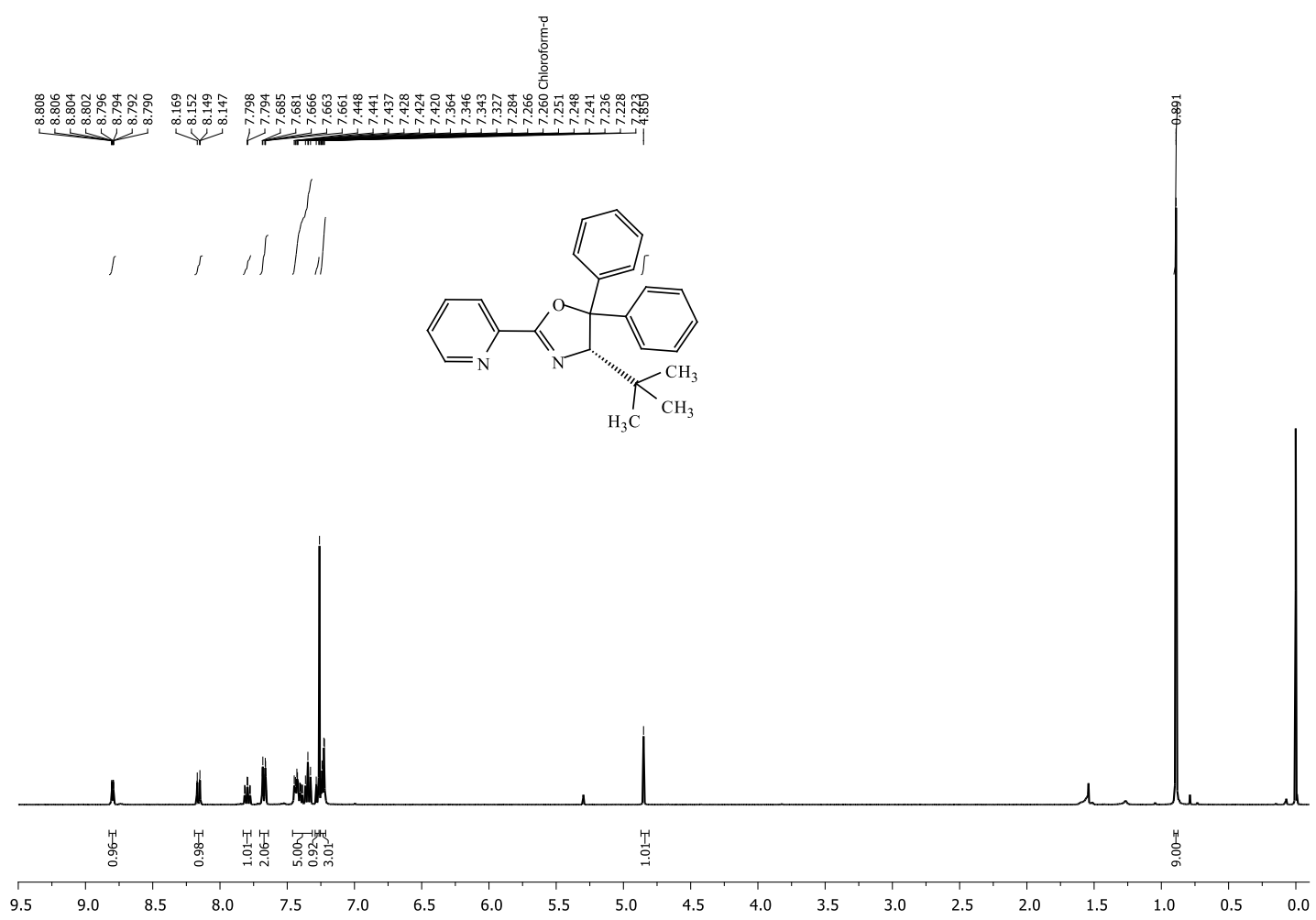

${ }^{13} \mathrm{C}$ NMR (101 MHz, $\left.\mathrm{CDCl}_{3}\right)$

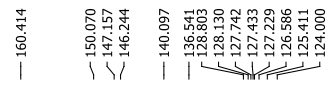

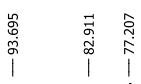

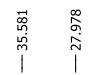
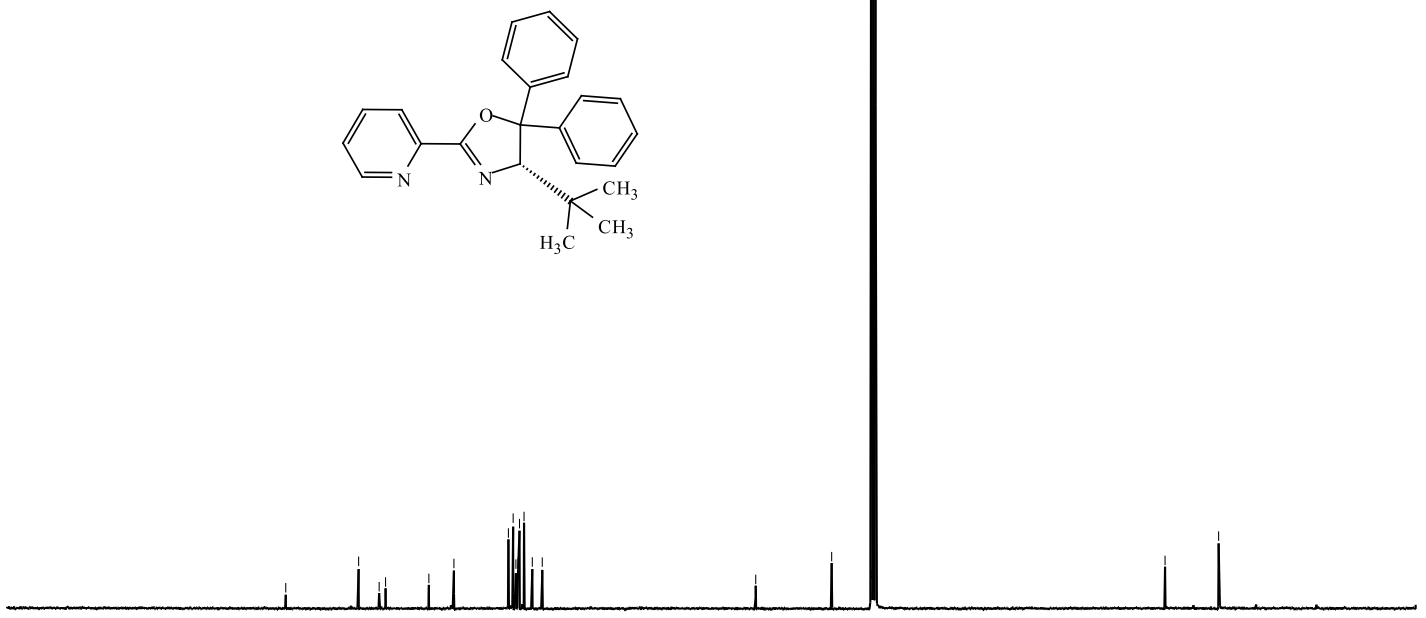

$\begin{array}{lllllllllll}200 & 190 & 180 & 170 & 160 & 150 & 140 & 130 & 120 & 110 & 100\end{array}$

Page S43 of S104 
(S)-4-(tert-Butyl)-2-(3-chloropyridin-2-yl)-4,5-dihydrooxazole (L1)

${ }^{1} \mathrm{H}$ NMR (400 MHz, $\mathrm{CDCl}_{3}$ )
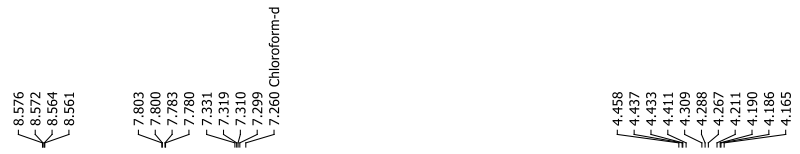

$1 / 1$

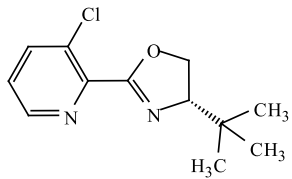

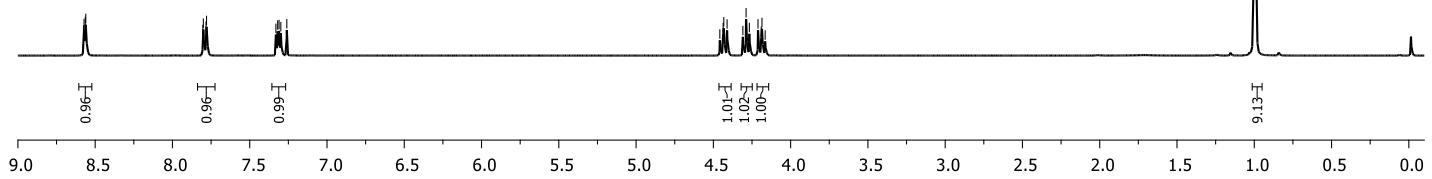

${ }^{13} \mathrm{C}$ NMR (101 MHz, $\left.\mathrm{CDCl}_{3}\right)$

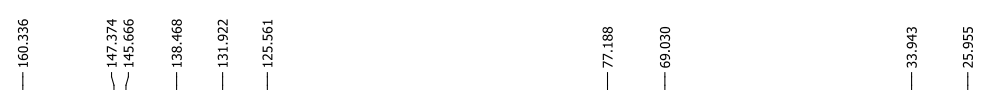
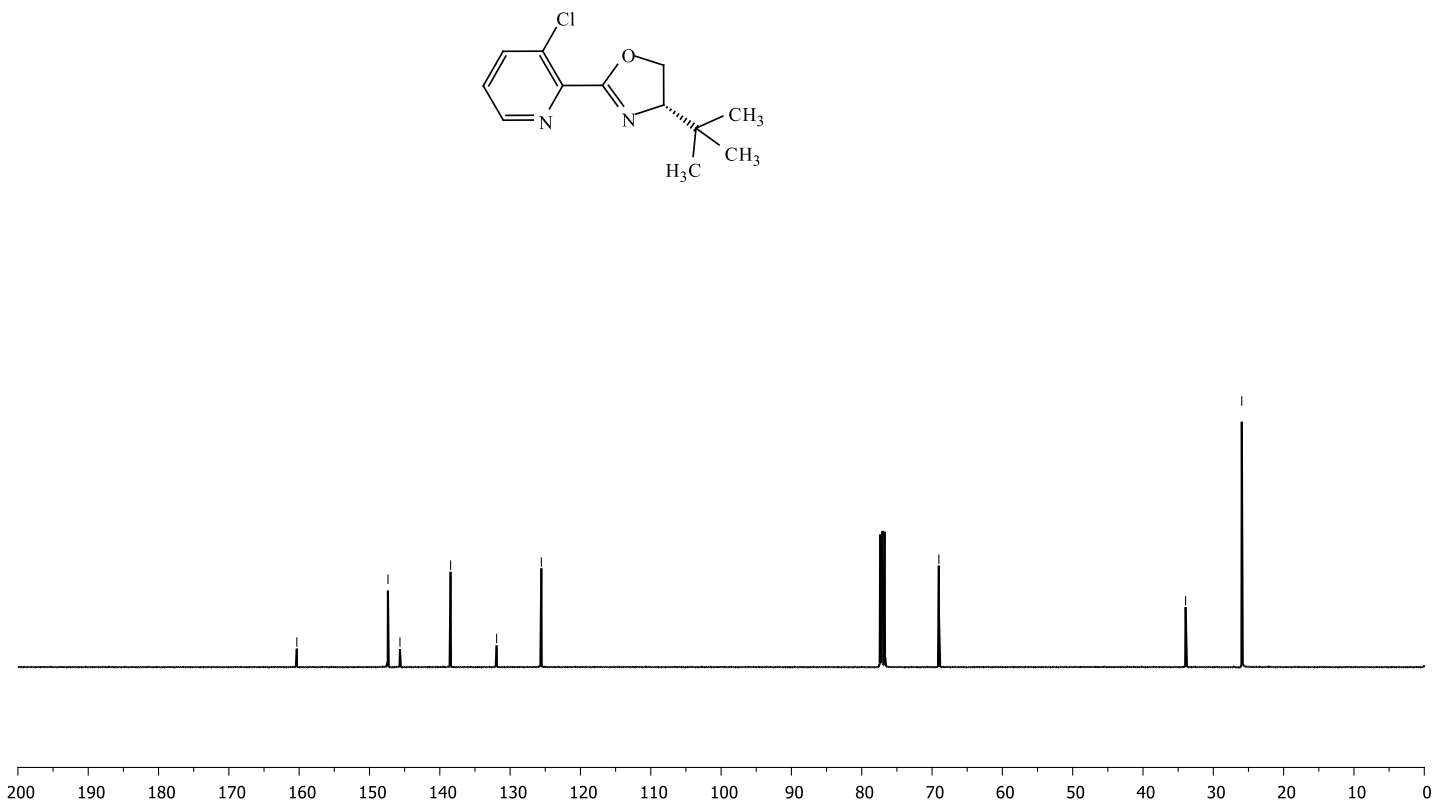

Page S44 of S104 
(S)-2-(3-Bromopyridin-2-yl)-4-(tert-butyl)-4,5-dihydrooxazole (SL2)

${ }^{1} \mathrm{H}$ NMR (400 MHz, $\mathrm{CDCl}_{3}$ )
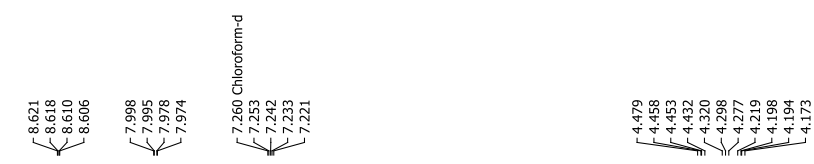

$1 / 1$
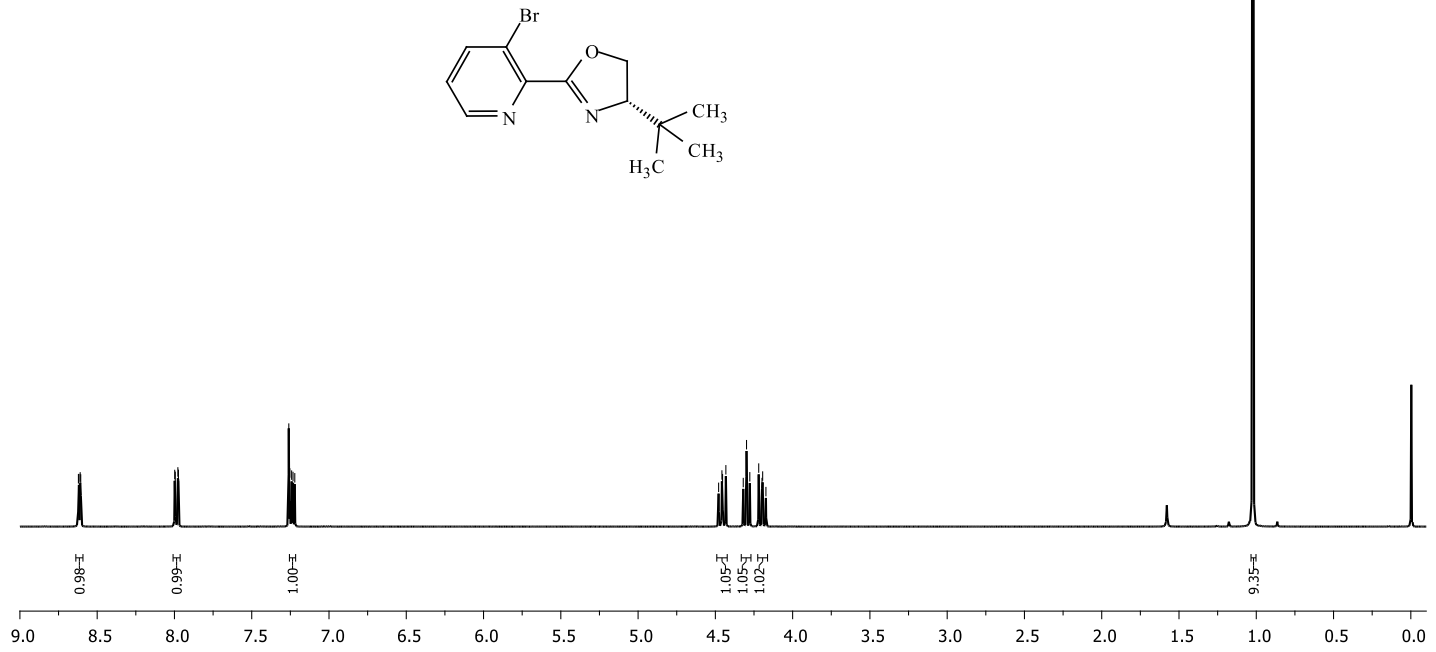

${ }^{13} \mathrm{C}$ NMR (101 MHz, $\left.\mathrm{CDCl}_{3}\right)$

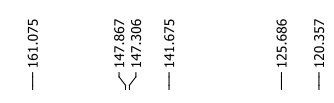
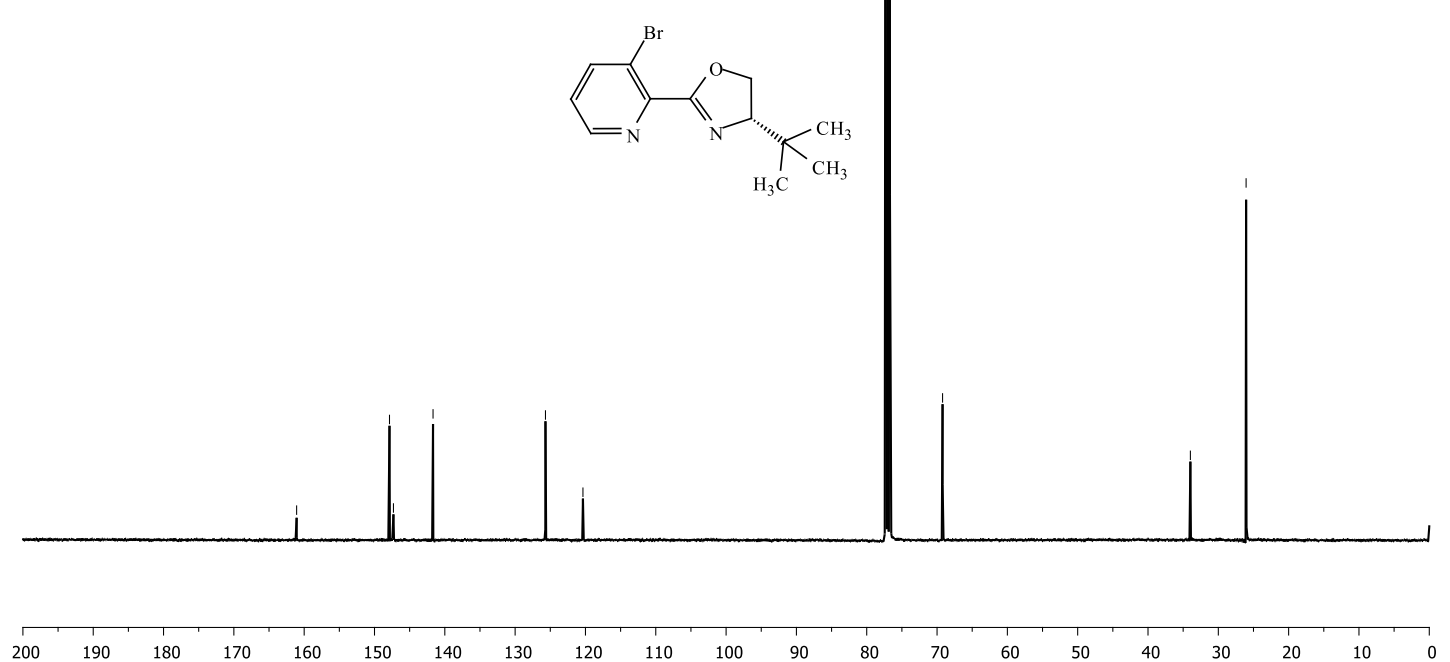

Page S45 of S104 
(S)-4-(tert-Butyl)-2-(3-phenylpyridin-2-yl)-4,5-dihydrooxazole (SL3)

${ }^{1} \mathrm{H}$ NMR (400 MHz, $\left.\mathrm{CDCl}_{3}\right)$

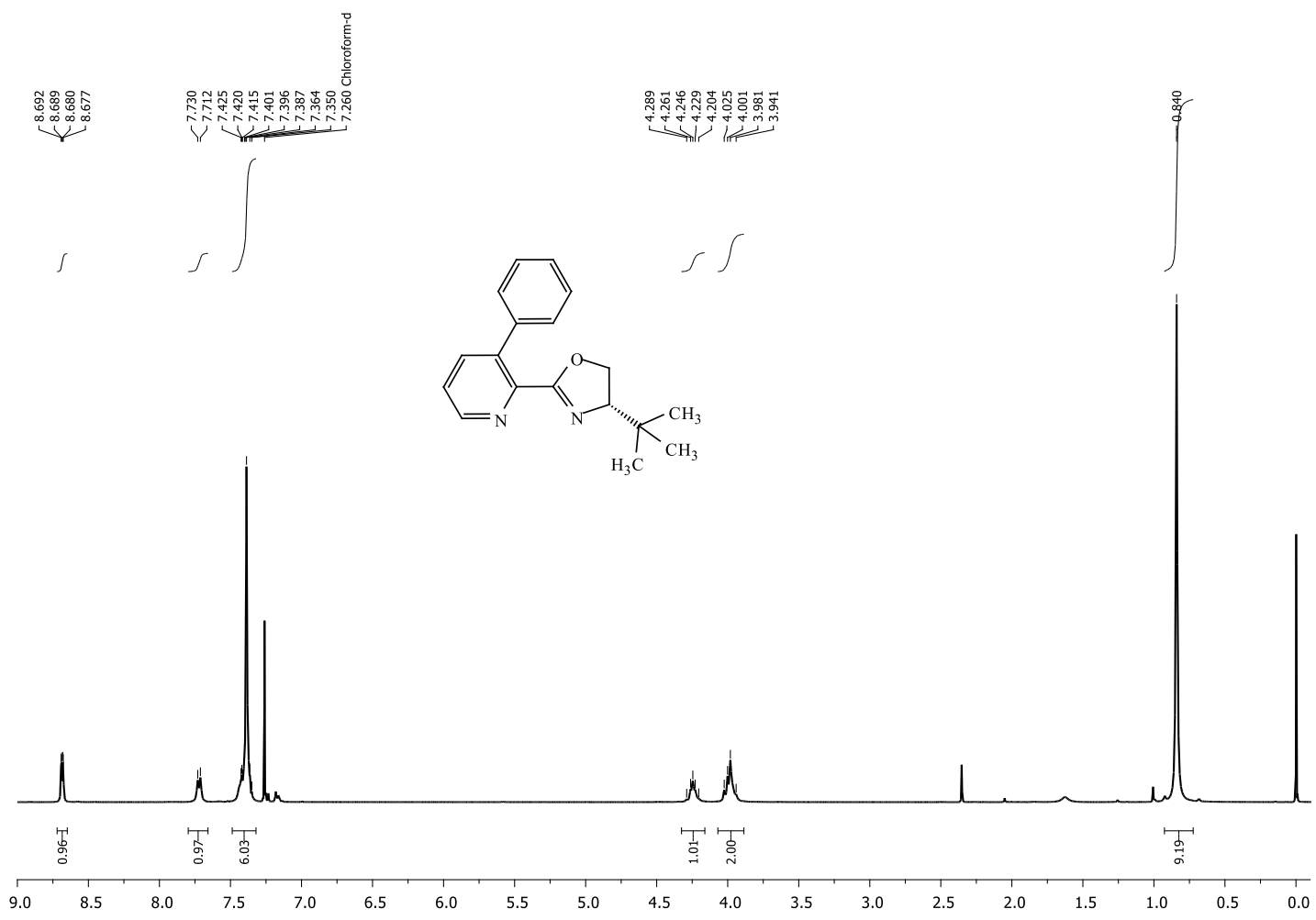

${ }^{13} \mathrm{C}$ NMR (101 MHz, $\left.\mathrm{CDCl}_{3}\right)$

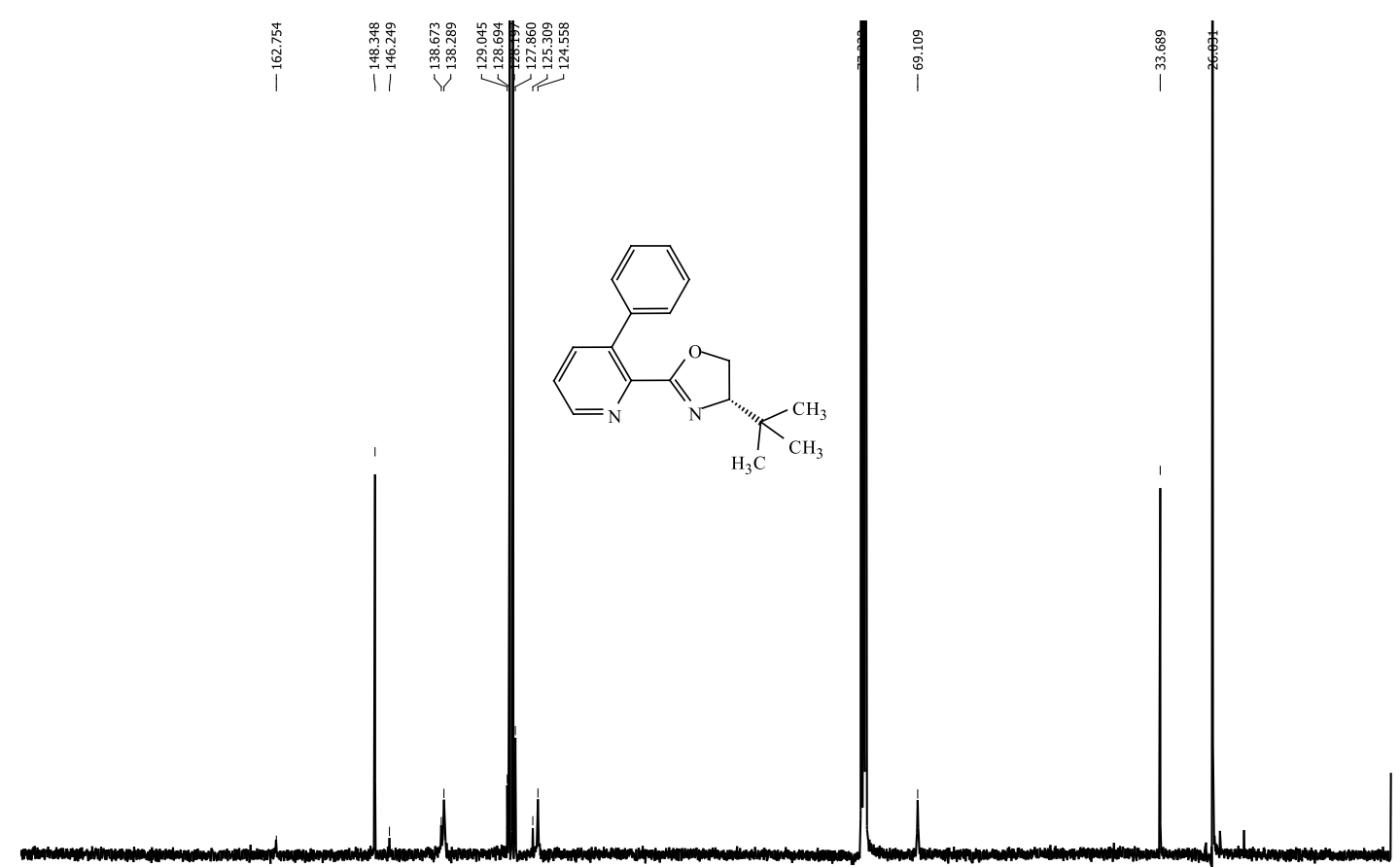

Page S46 of S104 
(S)-2-(3-(Benzyloxy)pyridin-2-yl)-4-(tert-butyl)-4,5-dihydrooxazole (SL4)

${ }^{1} \mathrm{H}$ NMR (400 MHz, $\left.\mathrm{CDCl}_{3}\right)$

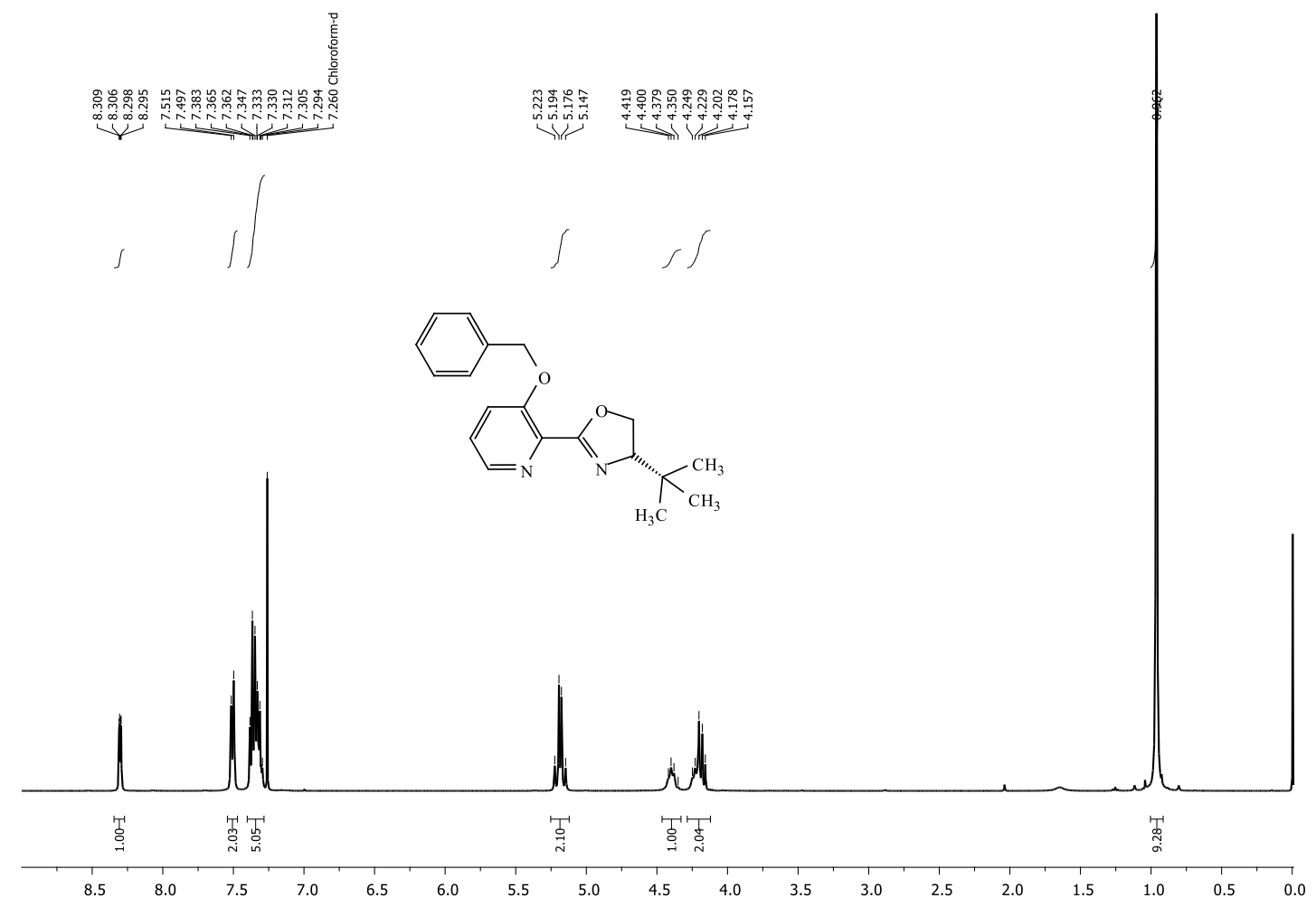

${ }^{13} \mathrm{C}$ NMR (101 MHz, $\left.\mathrm{CDCl}_{3}\right)$

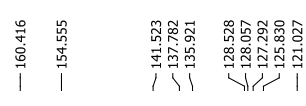

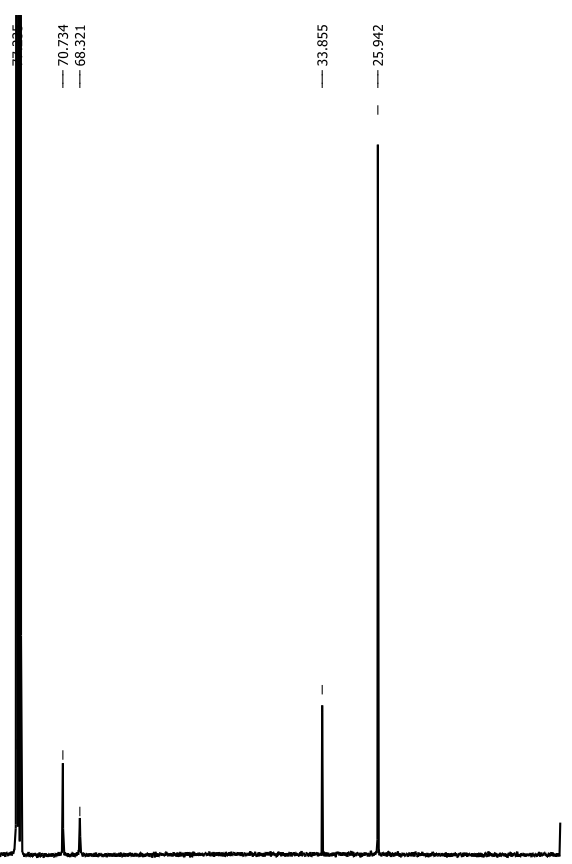

Page S47 of S104 
(S)-4-(tert-Butyl)-2-(3-(trifluoromethyl)pyridin-2-yl)-4,5-dihydrooxazole (SL5)

${ }^{1} \mathrm{H}$ NMR (400 MHz, $\left.\mathrm{CDCl}_{3}\right)$

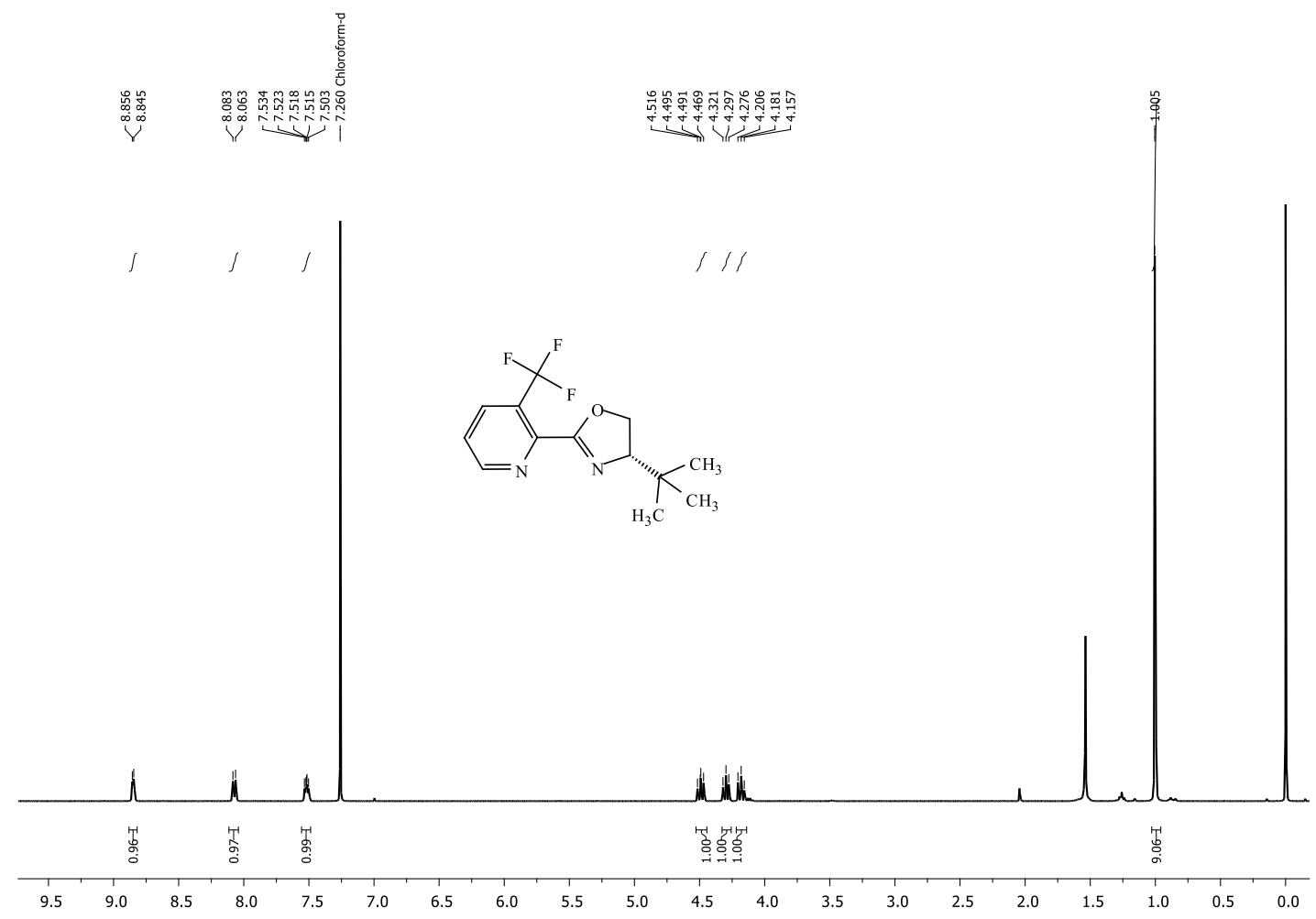

${ }^{13} \mathrm{C}$ NMR (101 MHz, $\left.\mathrm{CDCl}_{3}\right)$
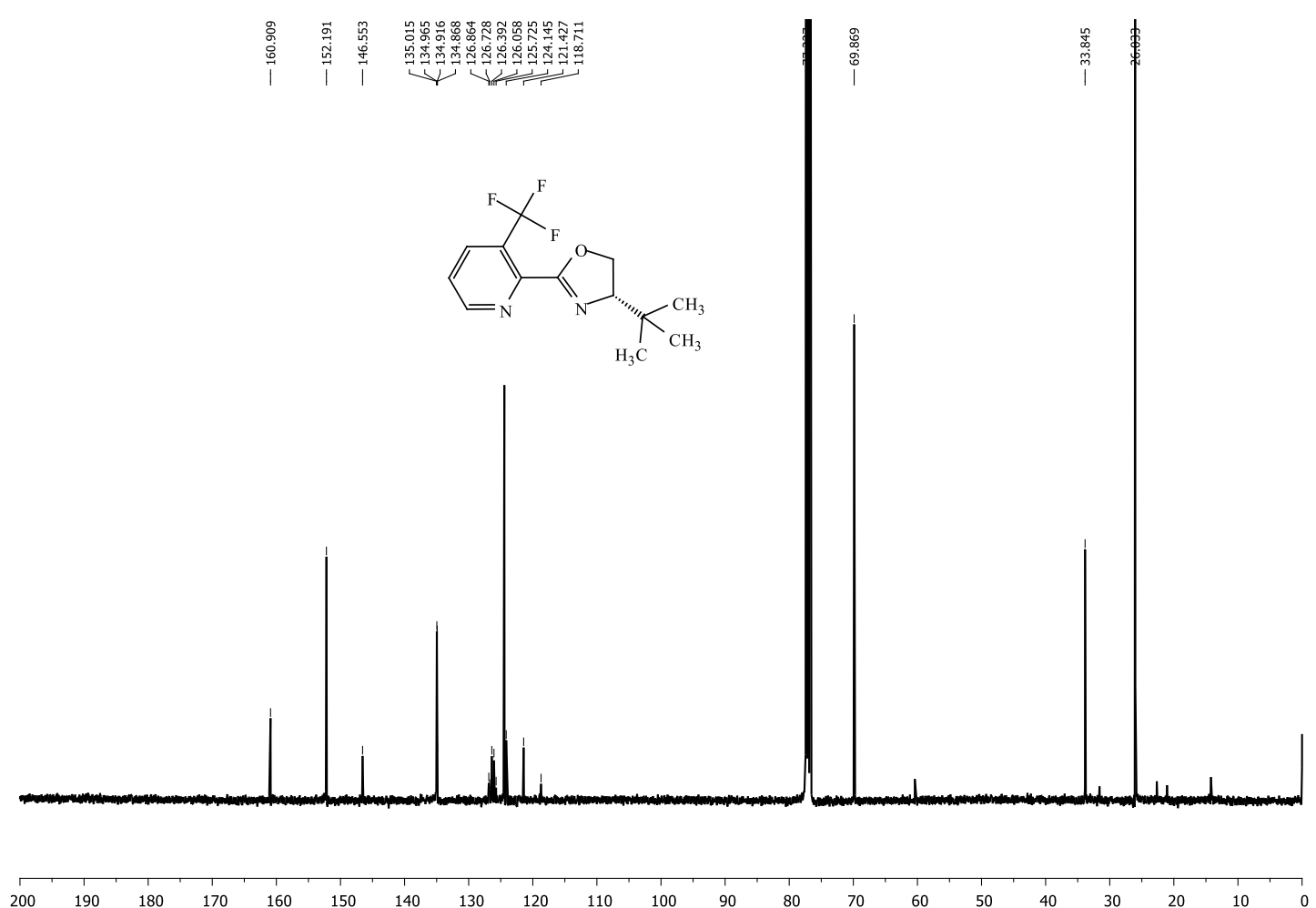

Page S48 of S104 
${ }^{19}$ F NMR (376 MHz, CDCl 3$)$
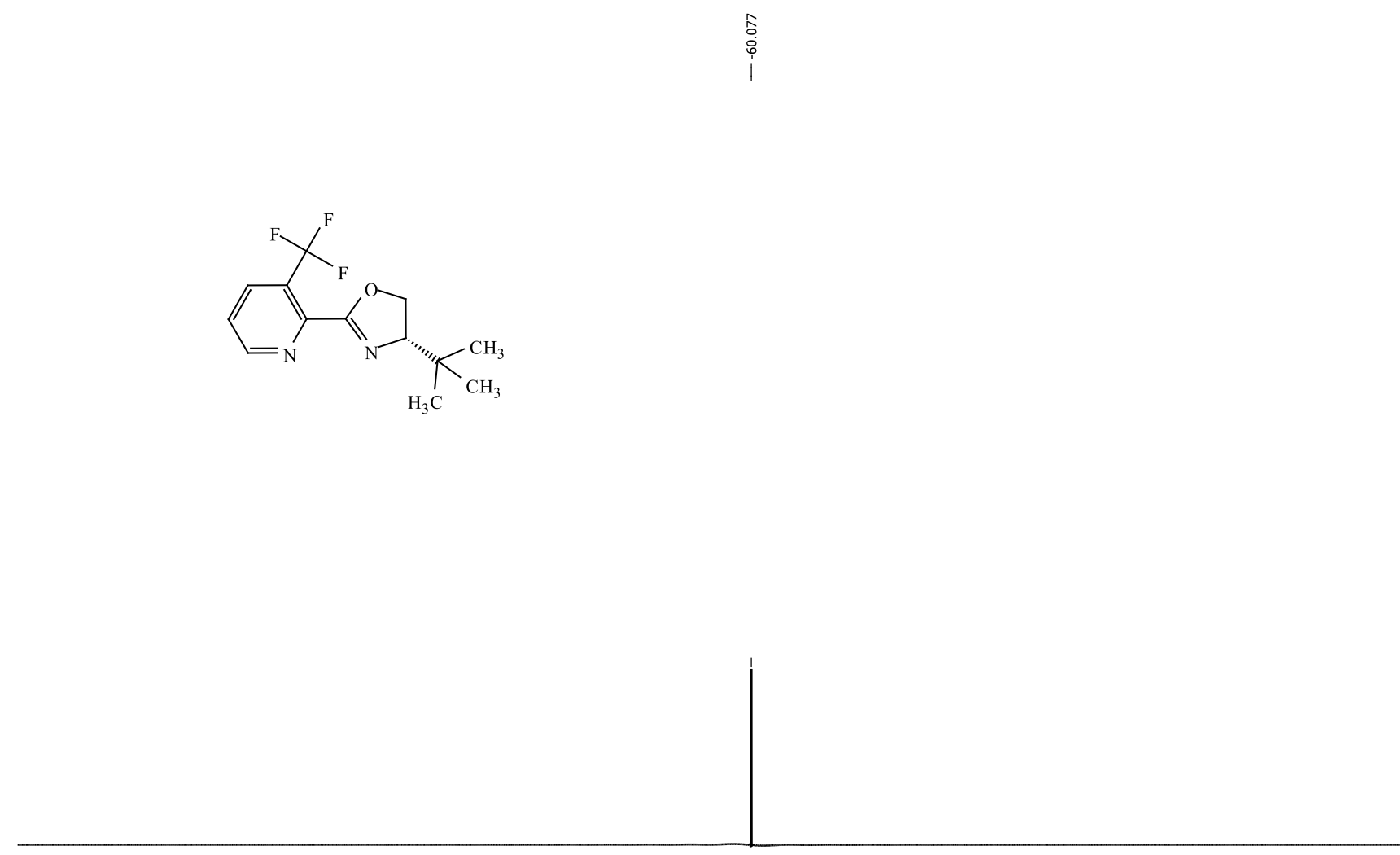

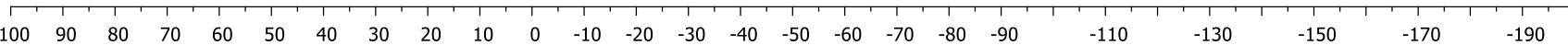


(S)-4-(tert-Butyl)-2-(5-methylpyridin-2-yl)-4,5-dihydrooxazole (SL6)

${ }^{1} \mathrm{H}$ NMR (400 MHz, $\left.\mathrm{CDCl}_{3}\right)$
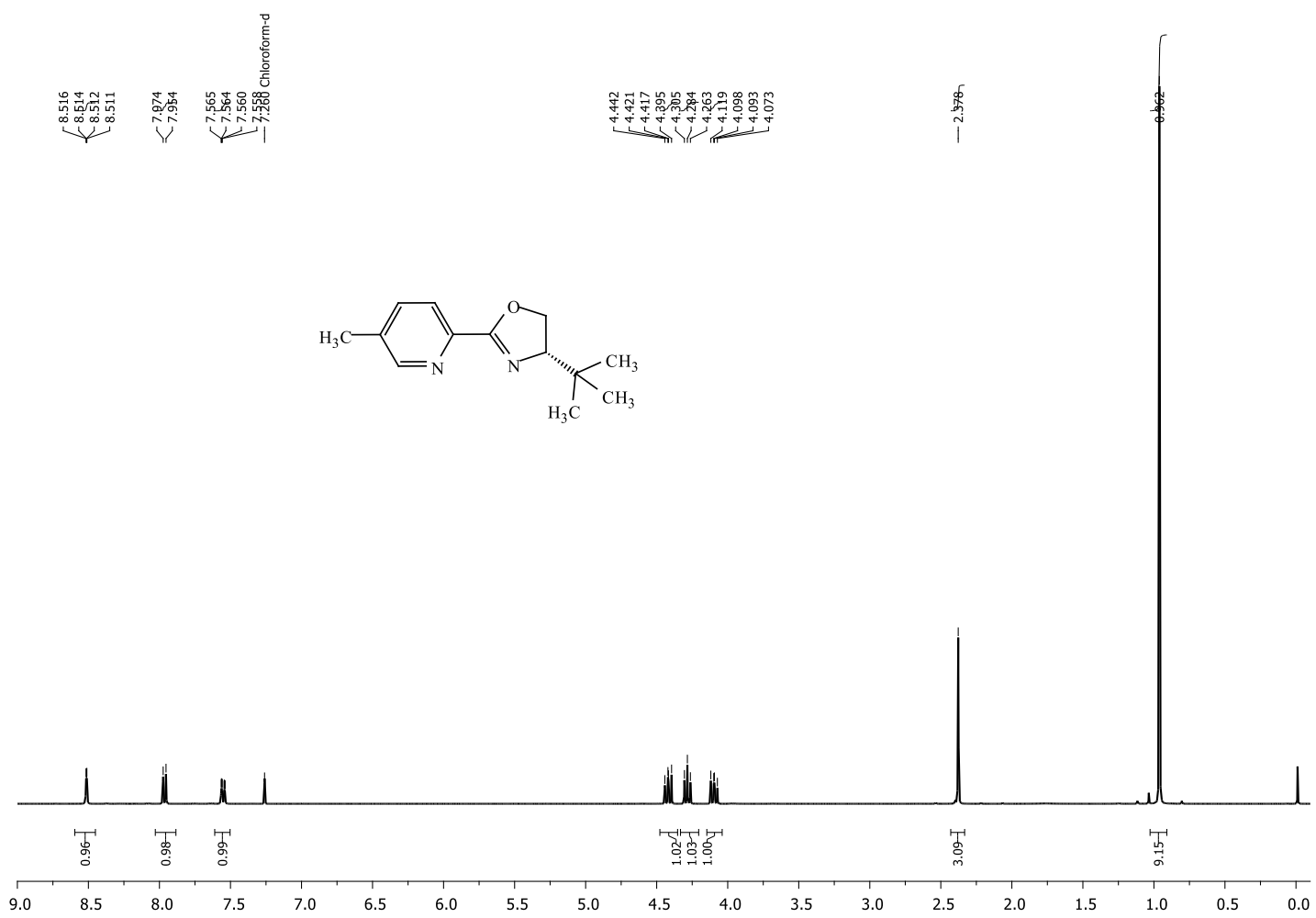

${ }^{13} \mathrm{C}$ NMR (101 MHz, $\left.\mathrm{CDCl}_{3}\right)$

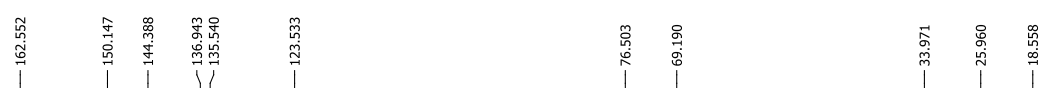
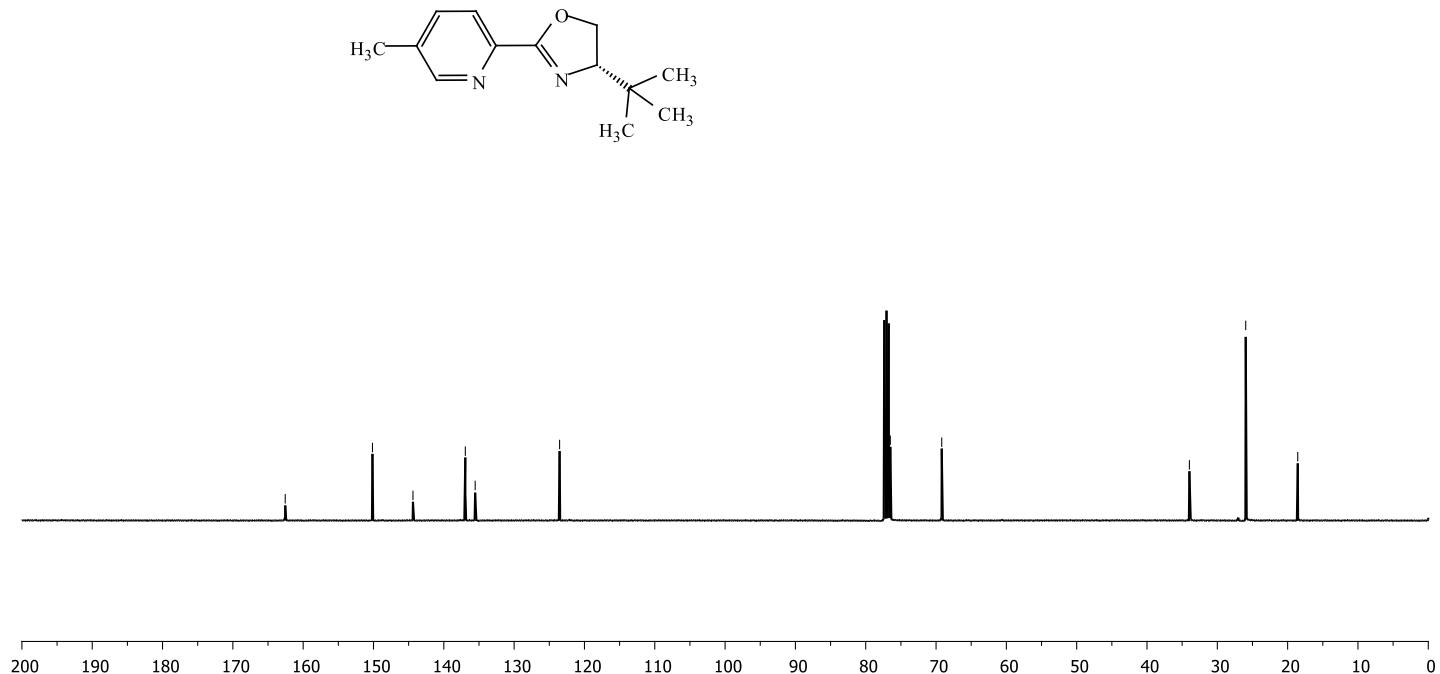

Page S50 of S104 
tert-Butyl 4,4-bis(4-methylbenzyl)-5-oxoisoxazolidine-2-carboxylate (Boc-1b)

${ }^{1} \mathrm{H}$ NMR (400 MHz, $\mathrm{CDCl}_{3}$ )

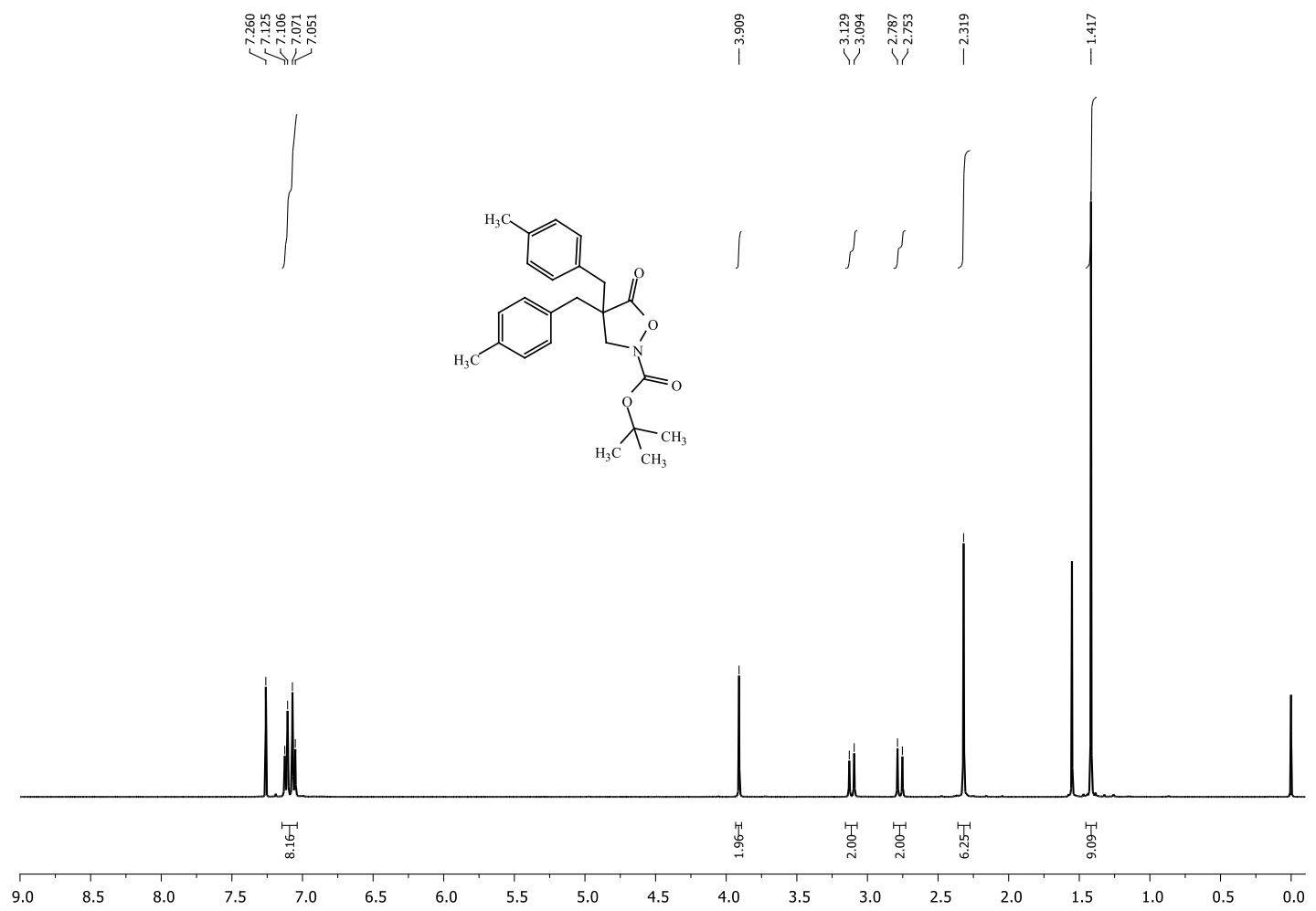

${ }^{13} \mathrm{C}$ NMR (101 MHz, $\left.\mathrm{CDCl}_{3}\right)$
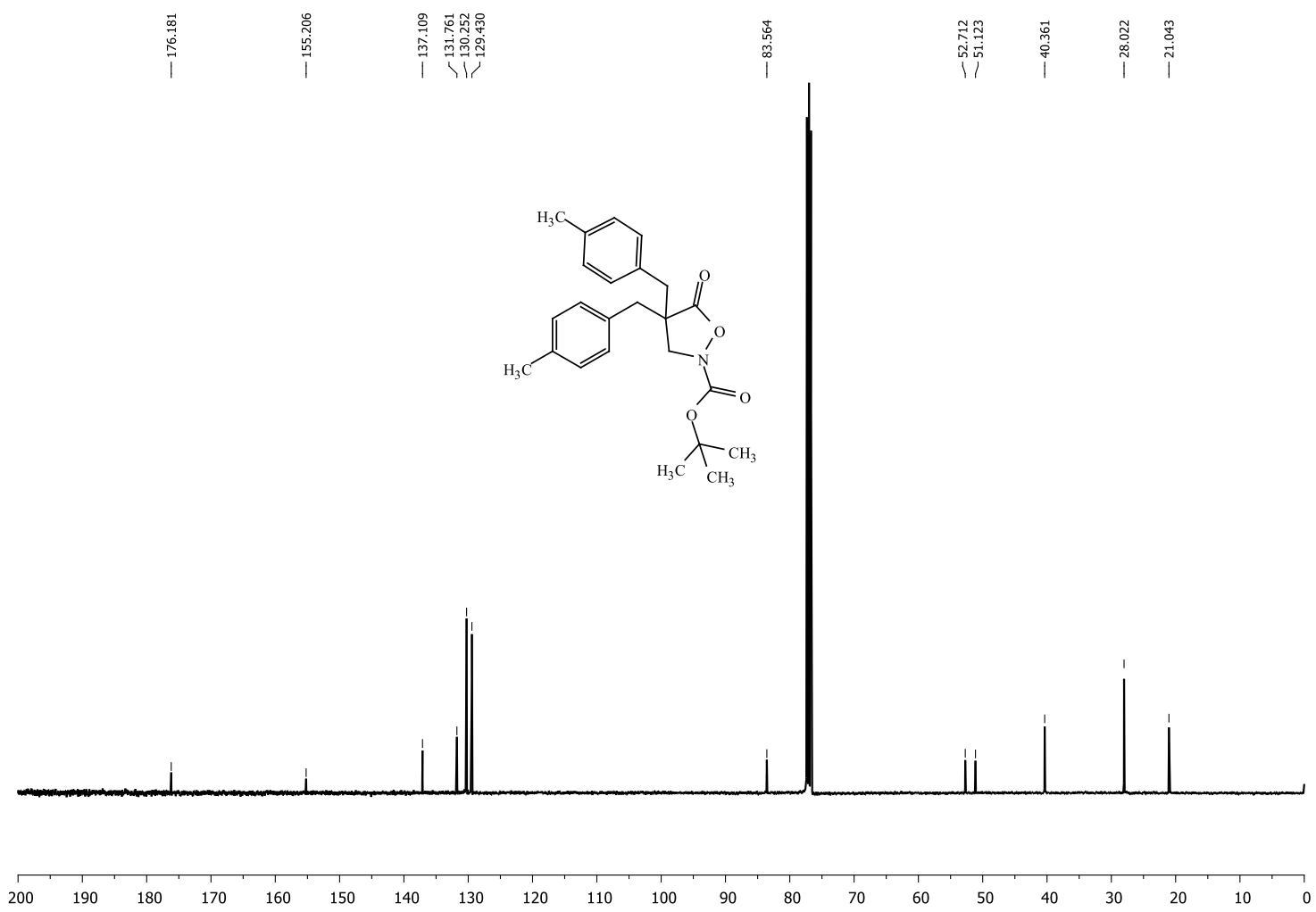

Page S51 of S104 
tert-Butyl 4,4-bis(2-methylbenzyl)-5-oxoisoxazolidine-2-carboxylate (Boc-1c)

${ }^{1} \mathrm{H}$ NMR (400 MHz, $\mathrm{CDCl}_{3}$ )

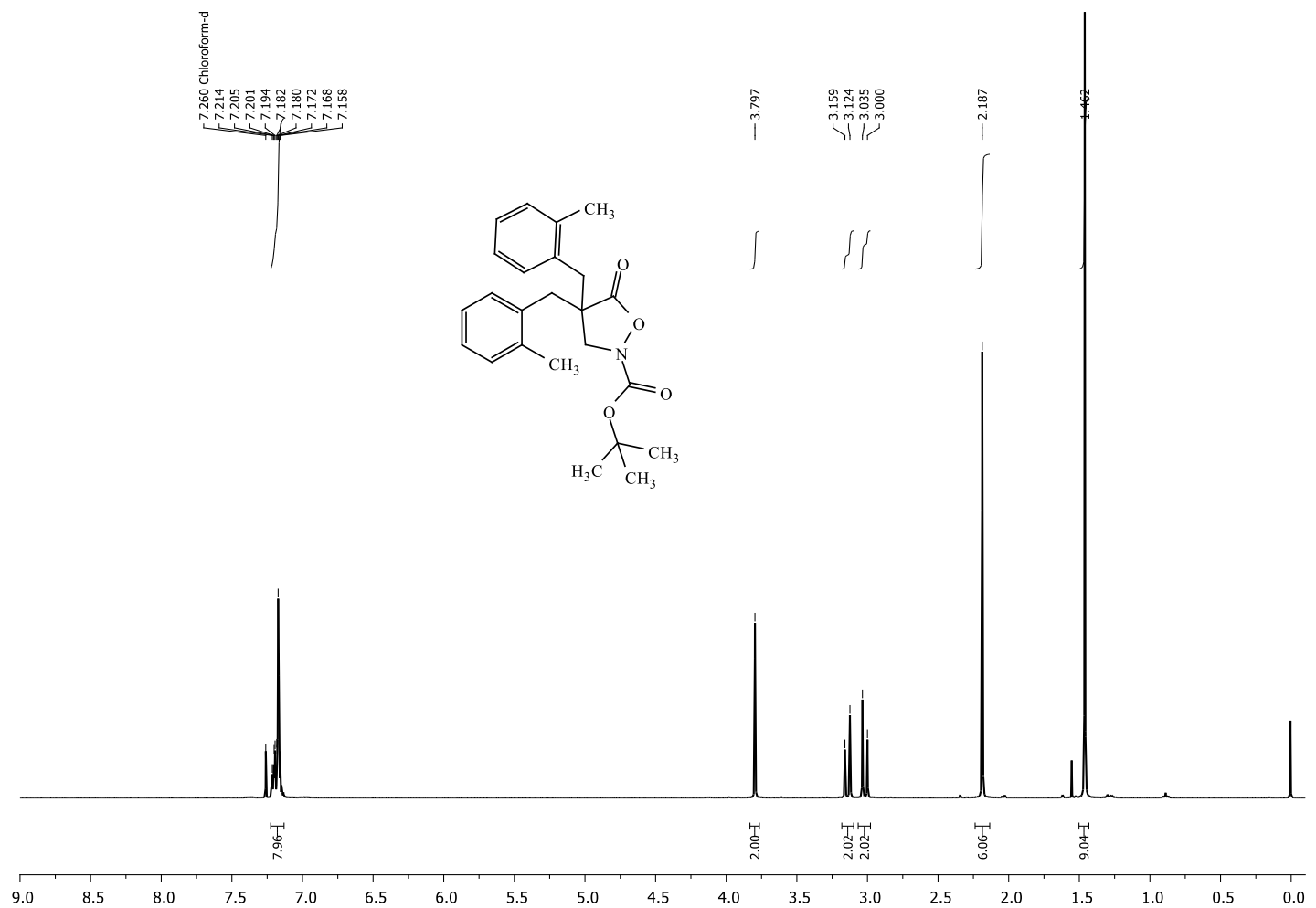

${ }^{13} \mathrm{C}$ NMR (101 MHz, $\left.\mathrm{CDCl}_{3}\right)$

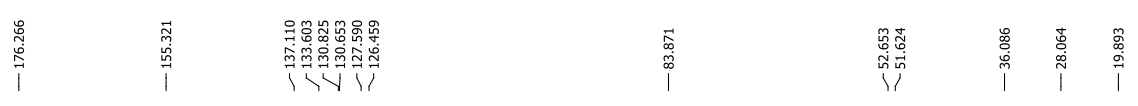
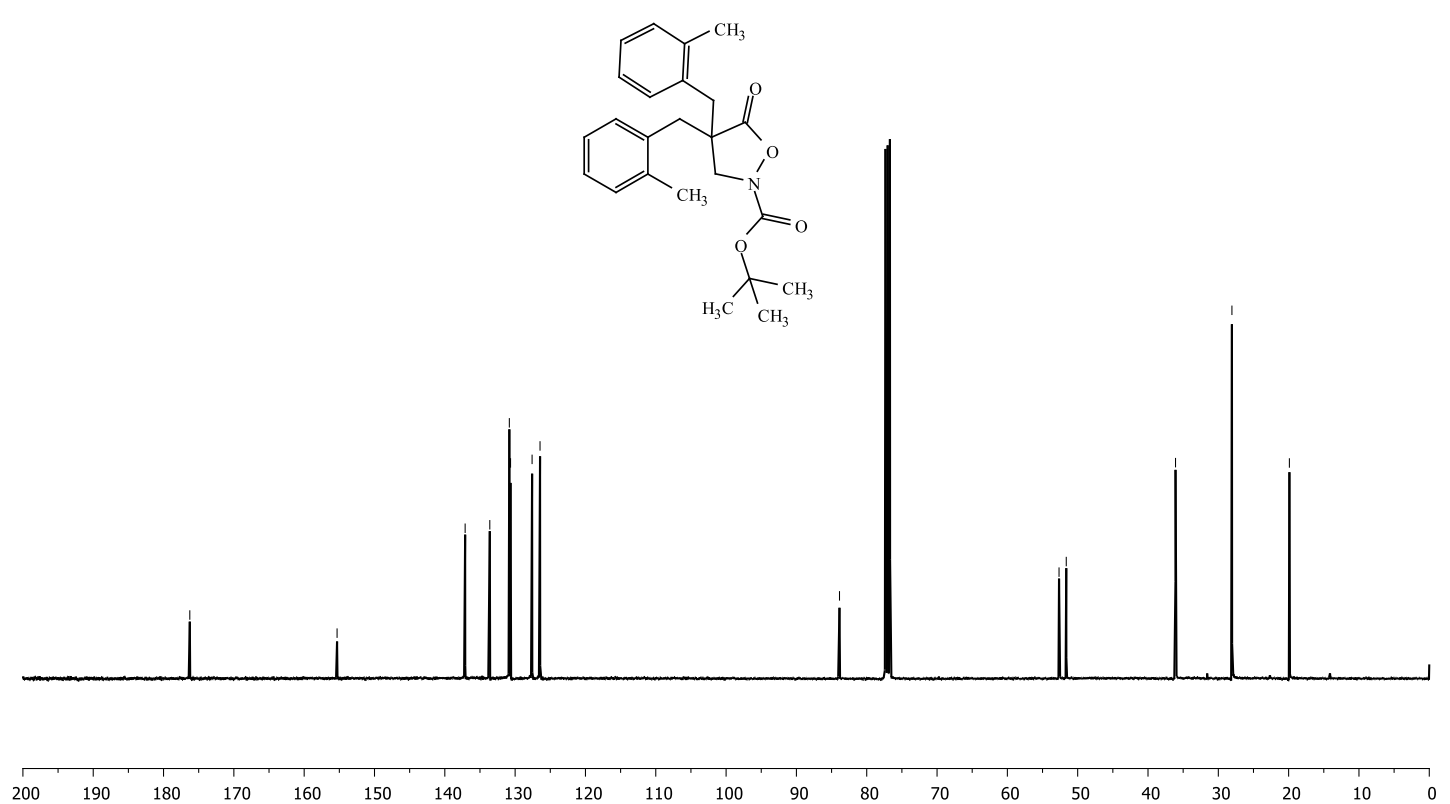

Page S52 of S104 
tert-Butyl 4,4-bis([1,1'-biphenyl]-4-ylmethyl)-5-oxoisoxazolidine-2-carboxylate (Boc-1d) ${ }^{1} \mathrm{H}$ NMR (400 MHz, $\mathrm{CDCl}_{3}$ )

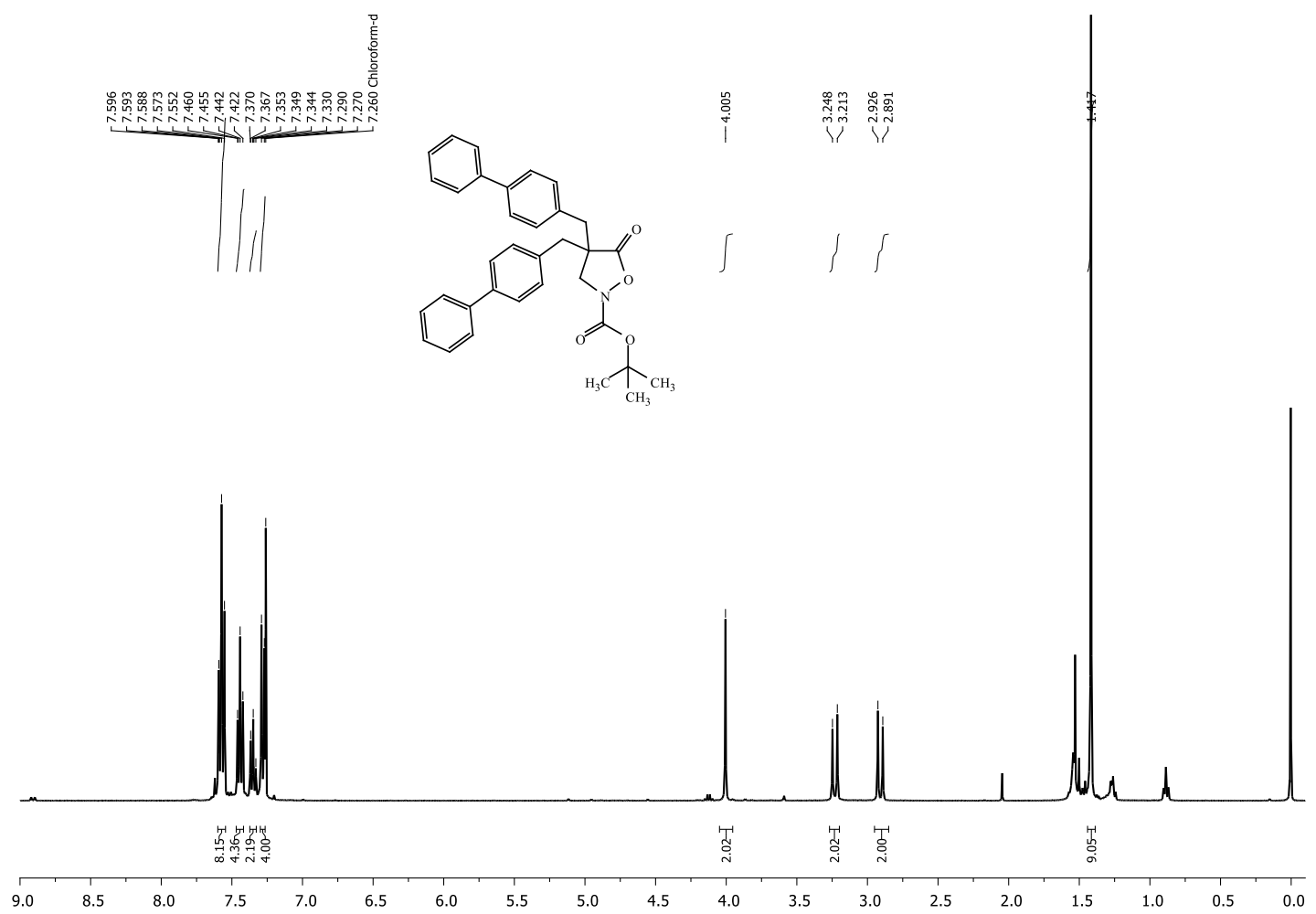

${ }^{13} \mathrm{C}$ NMR (101 MHz, $\left.\mathrm{CDCl}_{3}\right)$

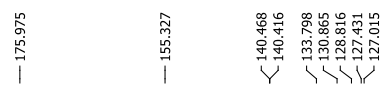
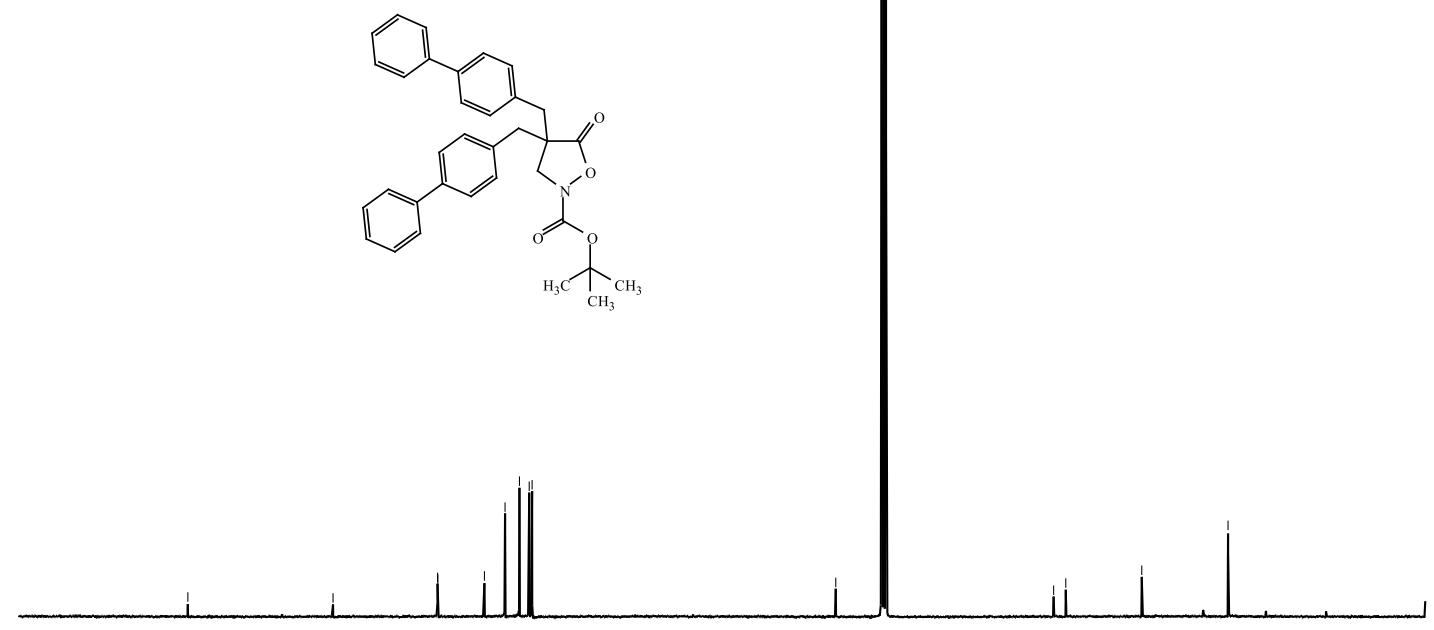

Page S53 of S104 
tert-Butyl 4,4-bis(4-(tert-butyl)benzyl)-5-oxoisoxazolidine-2-carboxylate (Boc-1e)

${ }^{1} \mathrm{H}$ NMR (400 MHz, $\left.\mathrm{CDCl}_{3}\right)$

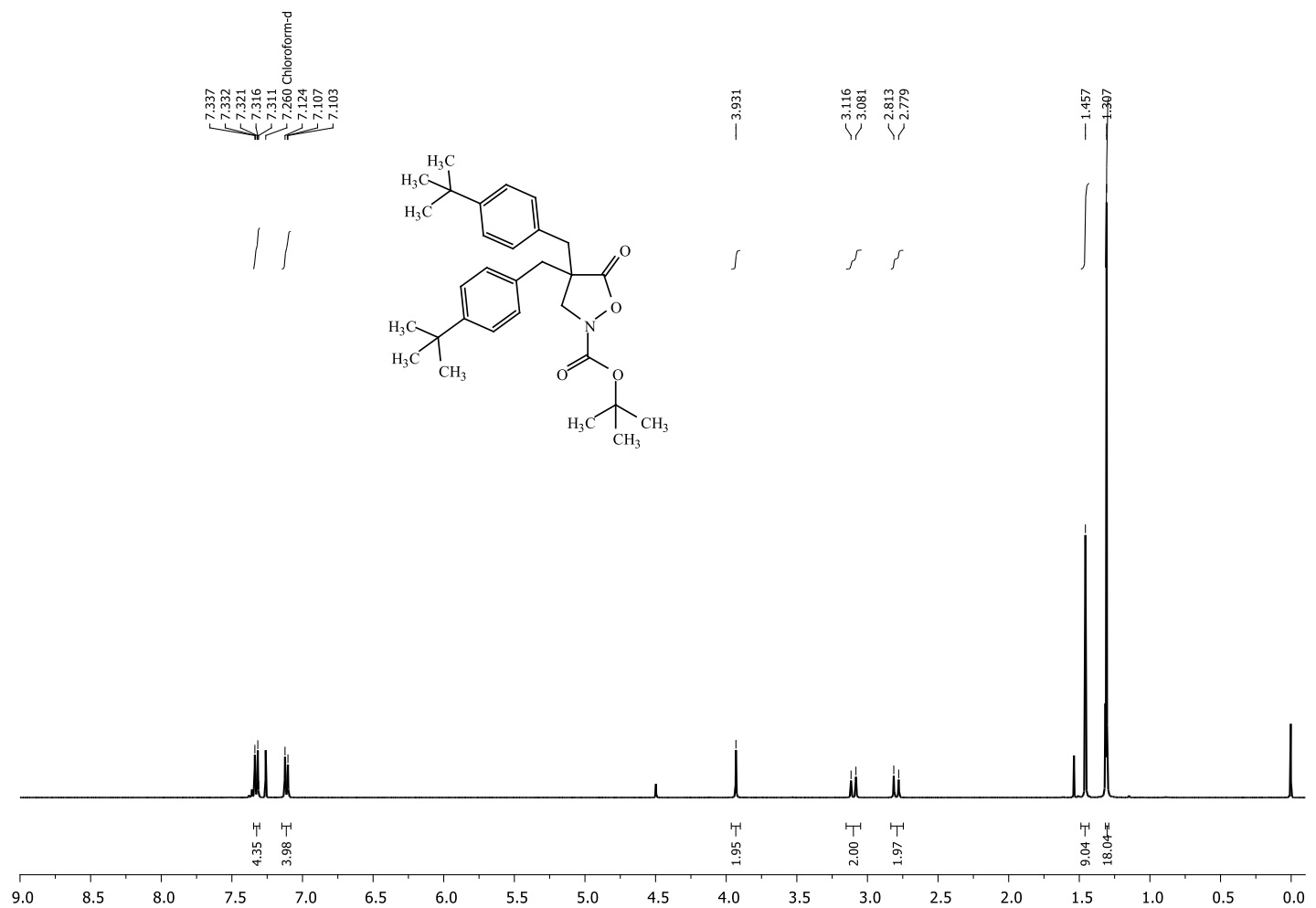

${ }^{13} \mathrm{C}$ NMR (101 MHz, $\left.\mathrm{CDCl}_{3}\right)$
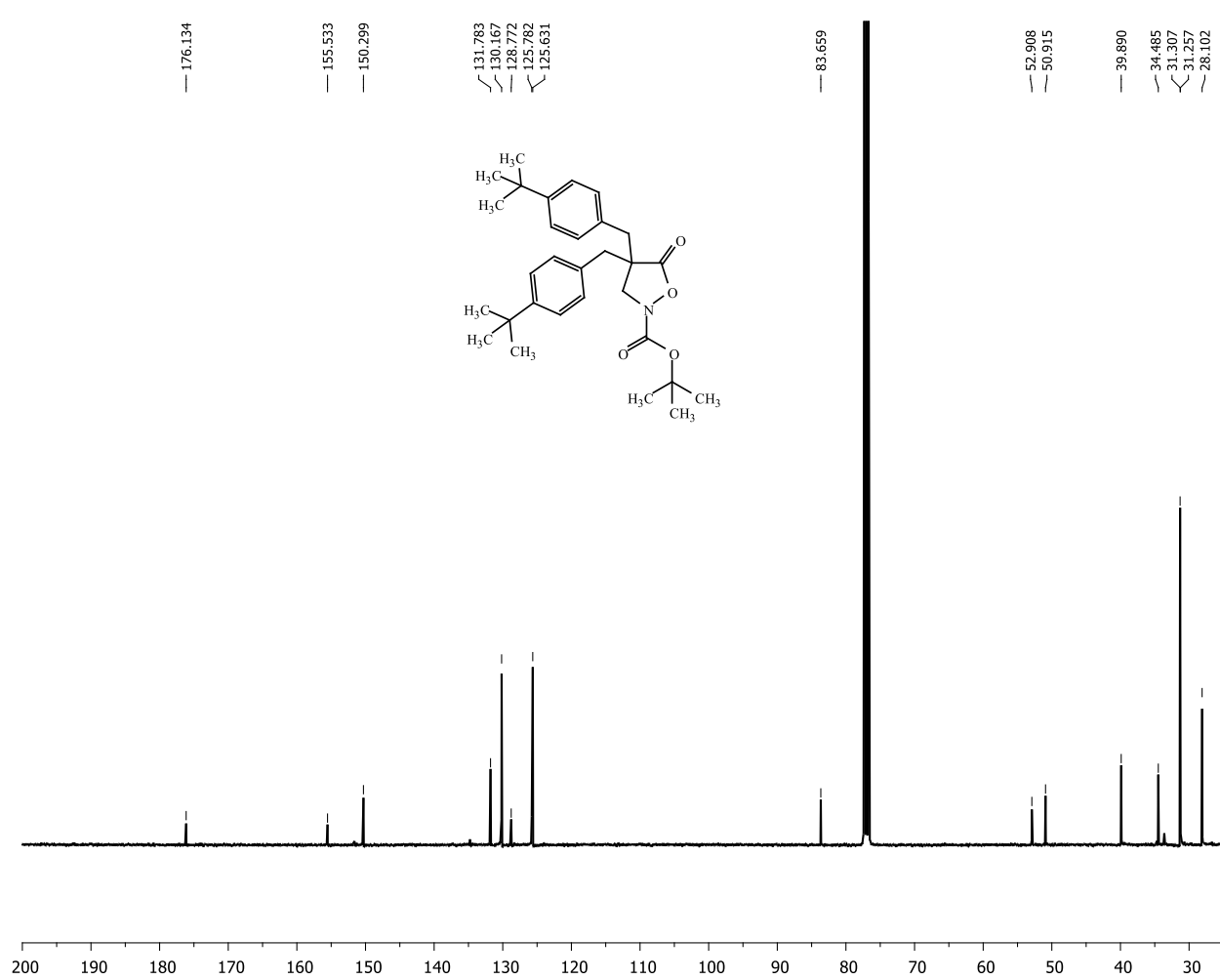

Page S54 of S104 
tert-Butyl 4,4-bis(4-bromobenzyl)-5-oxoisoxazolidine-2-carboxylate (Boc-1f)

${ }^{1} \mathrm{H}$ NMR (400 MHz, $\mathrm{CDCl}_{3}$ )

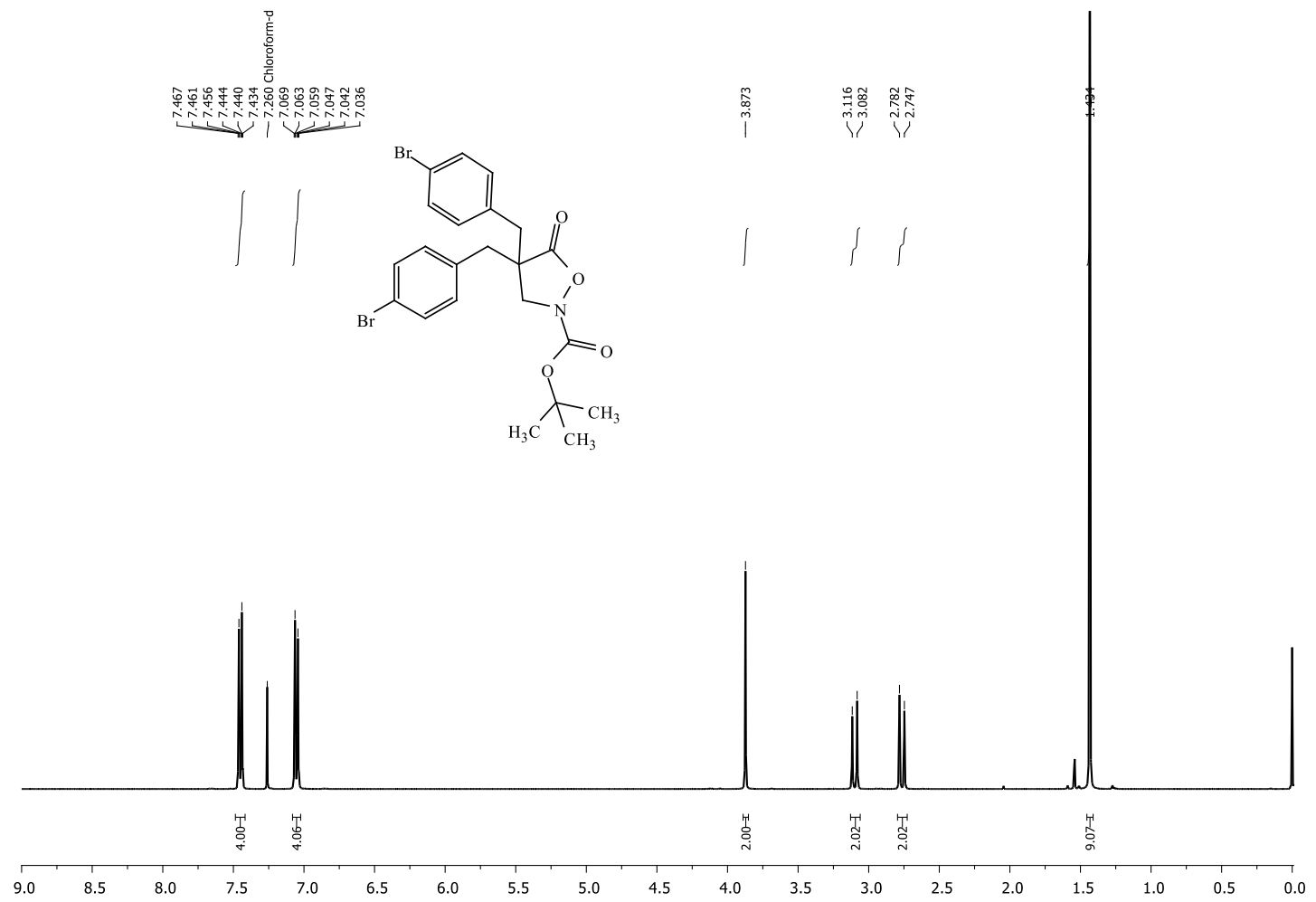

${ }^{13} \mathrm{C}$ NMR (101 MHz, $\left.\mathrm{CDCl}_{3}\right)$

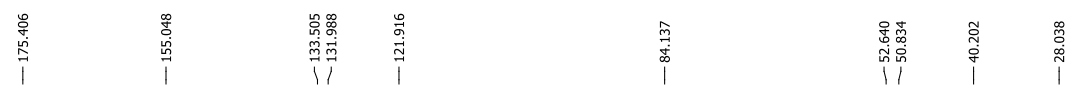

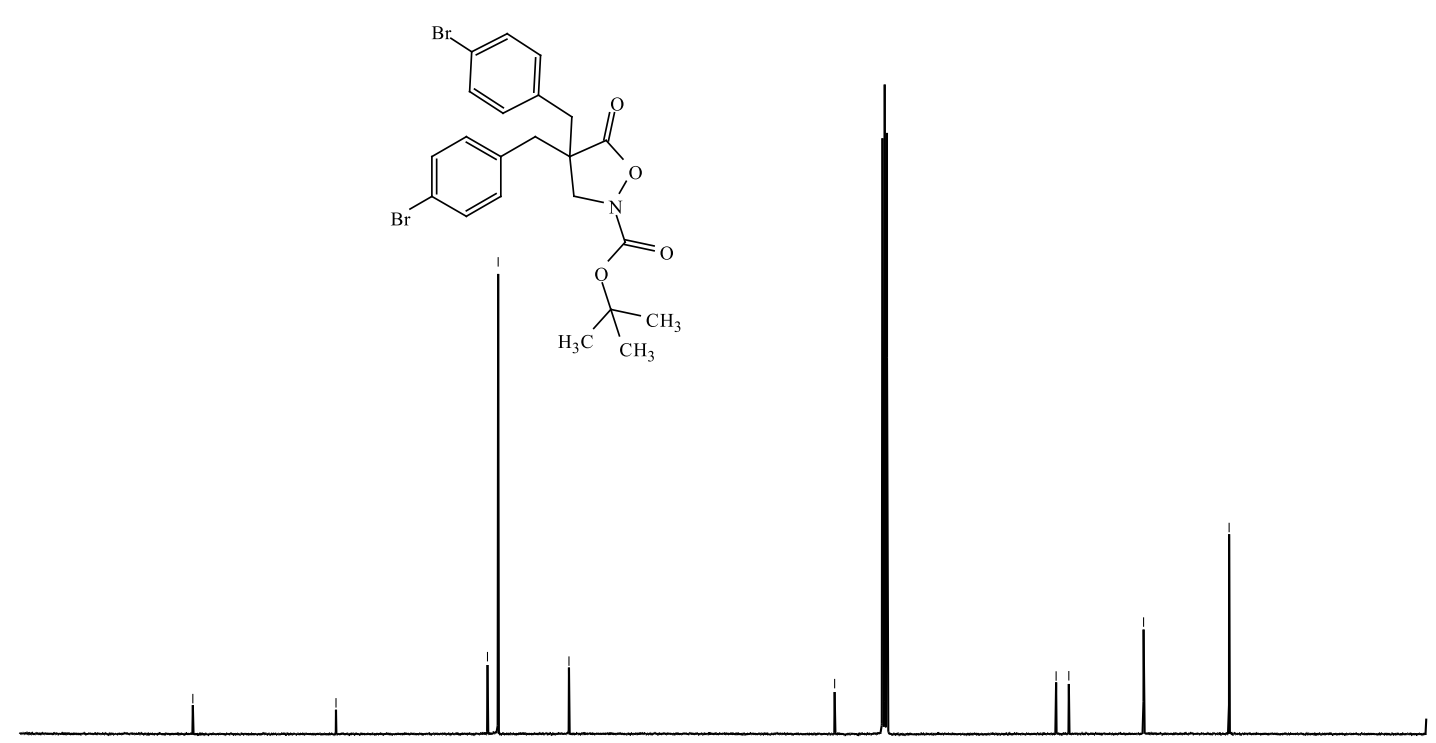

Page S55 of S104 
tert-Butyl 4,4-bis(2-chlorobenzyl)-5-oxoisoxazolidine-2-carboxylate (Boc-1g)

${ }^{1} \mathrm{H}$ NMR (400 MHz, $\left.\mathrm{CDCl}_{3}\right)$
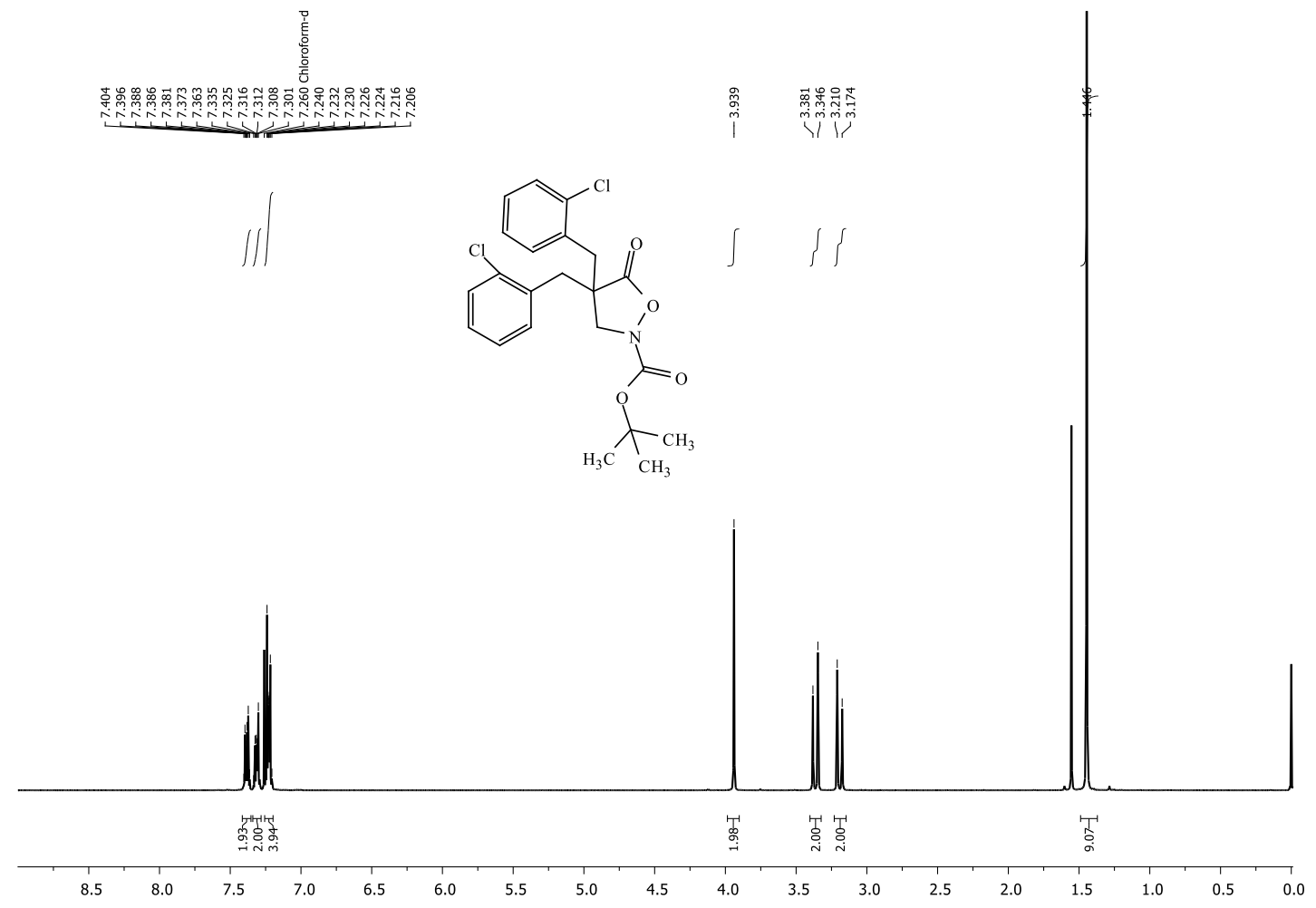

${ }^{13} \mathrm{C}$ NMR (101 MHz, $\left.\mathrm{CDCl}_{3}\right)$
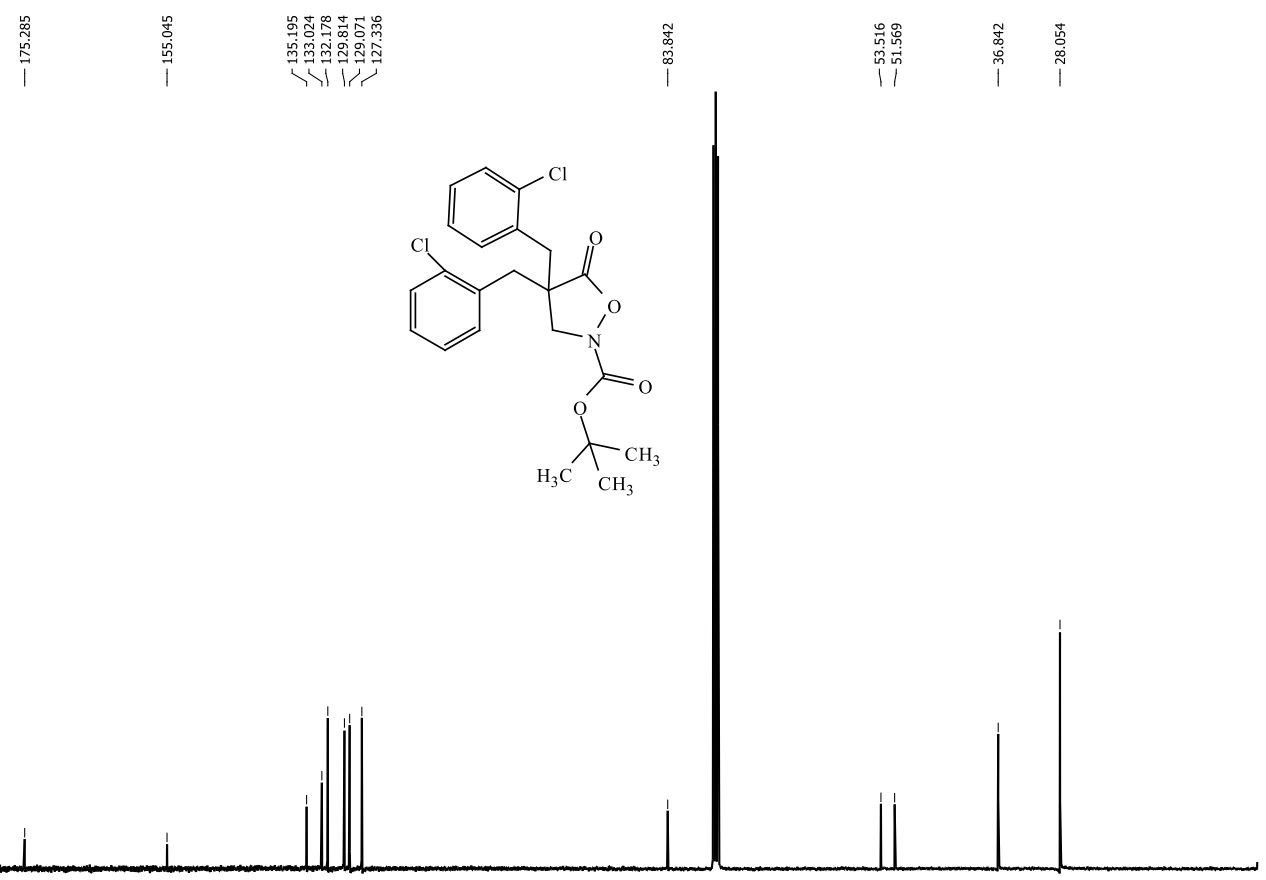

$200 \quad 190$

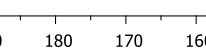

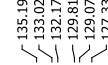
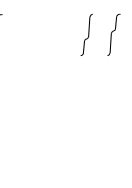
tert-Butyl 4-benzyl-4-(2-methylbenzyl)-5-oxoisoxazolidine-2-carboxylate (Boc-3c) ${ }^{1} \mathrm{H}$ NMR (400 MHz, $\left.\mathrm{CDCl}_{3}\right)$
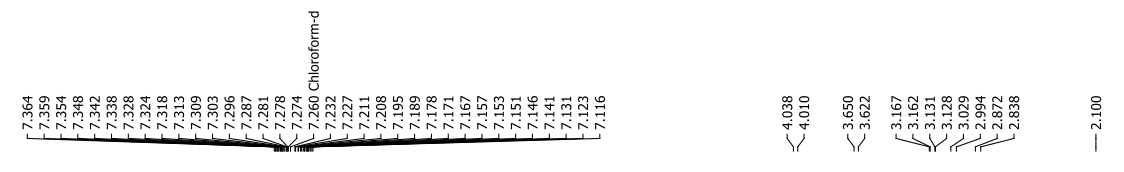

$\int 11$
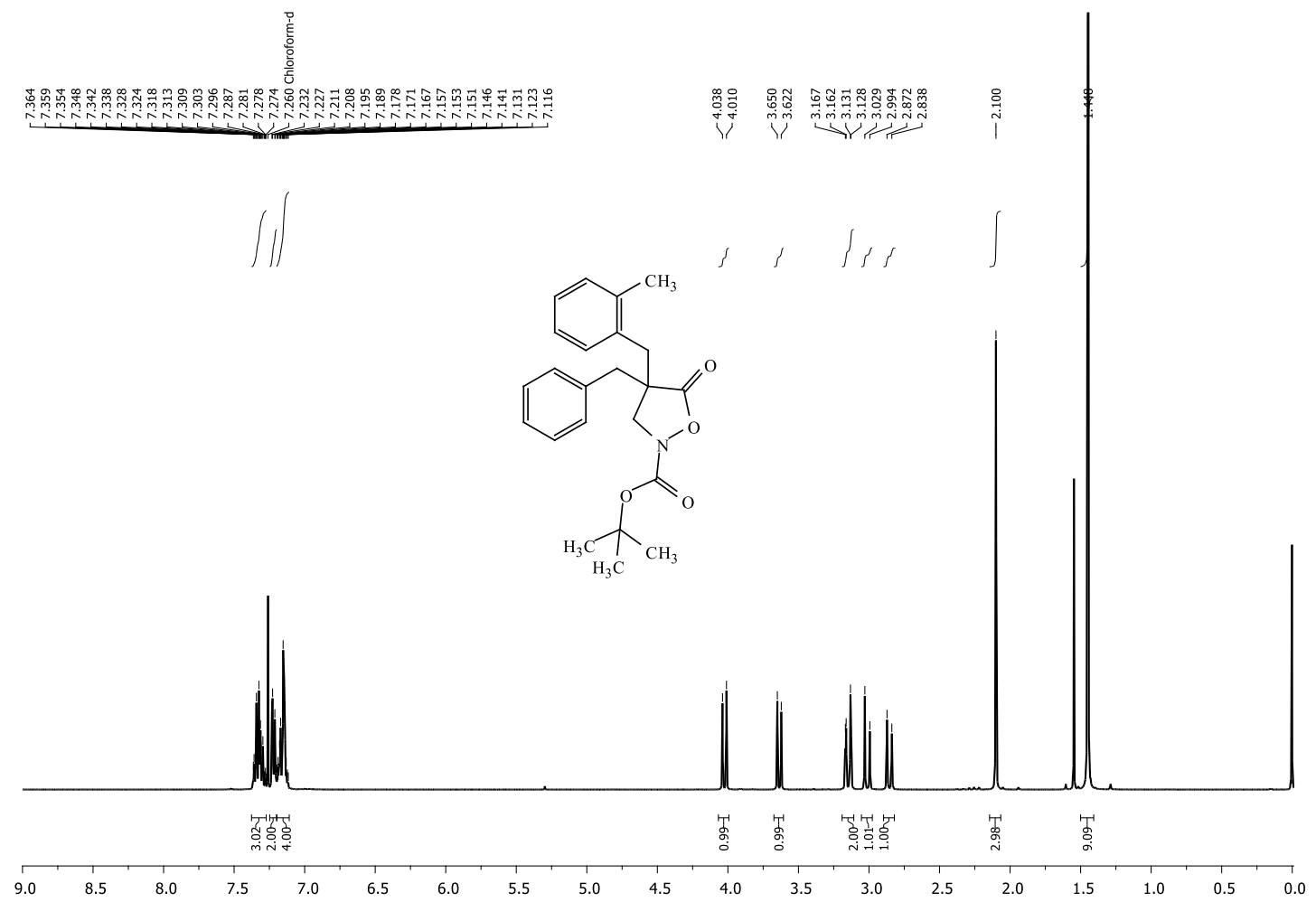

${ }^{13} \mathrm{C}$ NMR (101 MHz, $\left.\mathrm{CDCl}_{3}\right)$

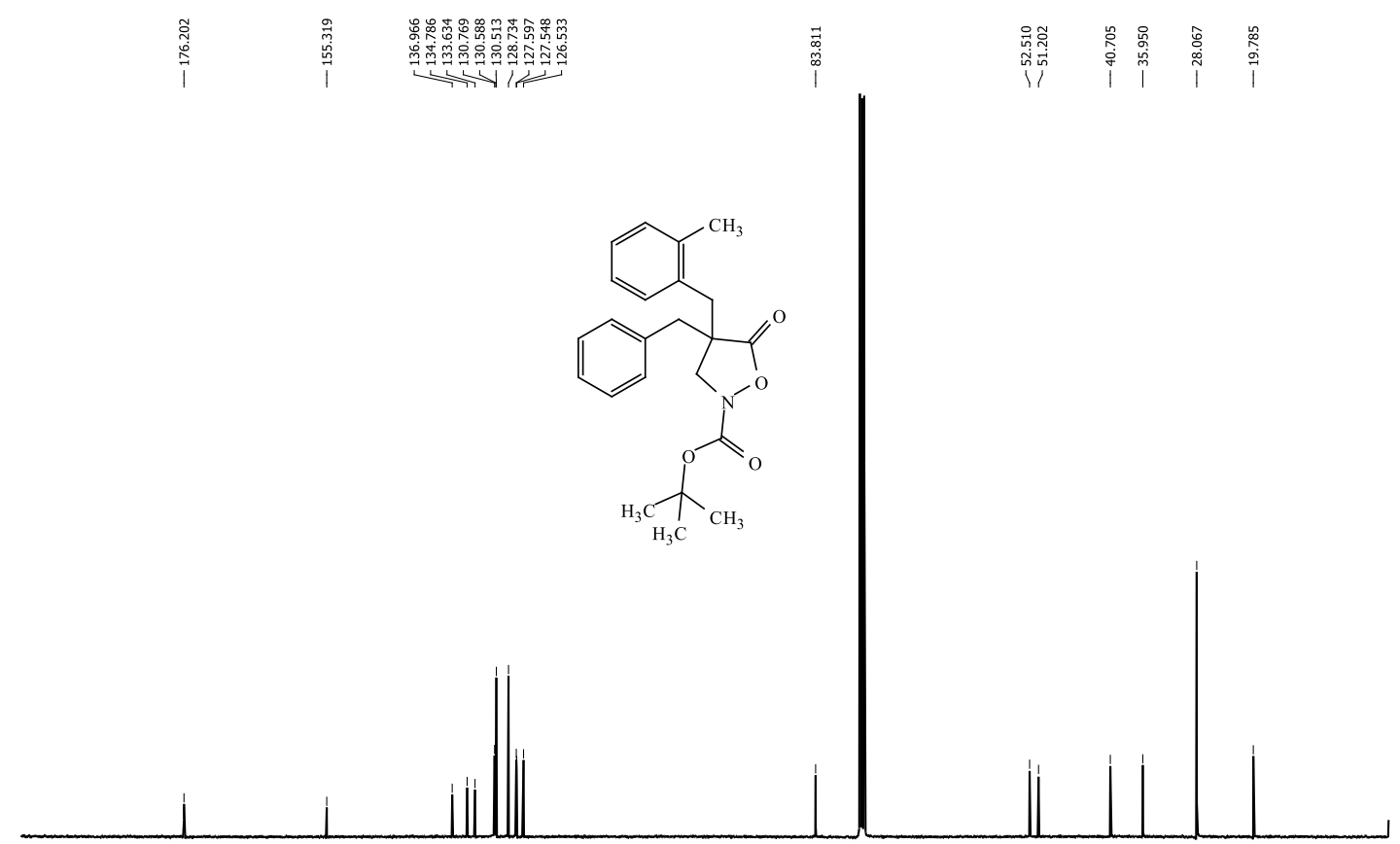


tert-Butyl 4-(2-methylbenzyl)-4-(4-methylbenzyl)-5-oxoisoxazolidine-2-carboxylate (Boc-3d) ${ }^{1} \mathrm{H}$ NMR (400 MHz, $\left.\mathrm{CDCl}_{3}\right)$

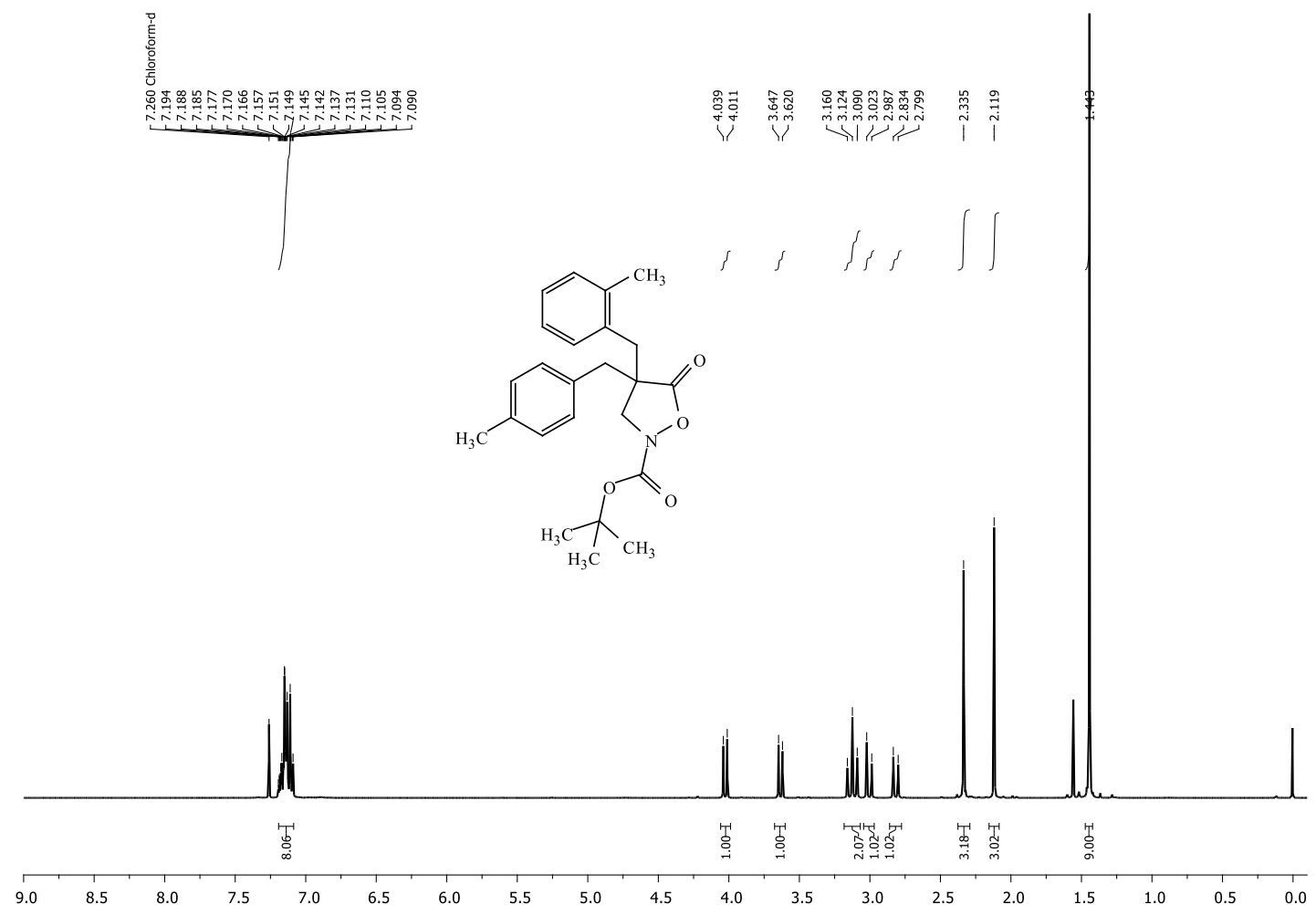

${ }^{13} \mathrm{C}$ NMR (101 MHz, $\left.\mathrm{CDCl}_{3}\right)$
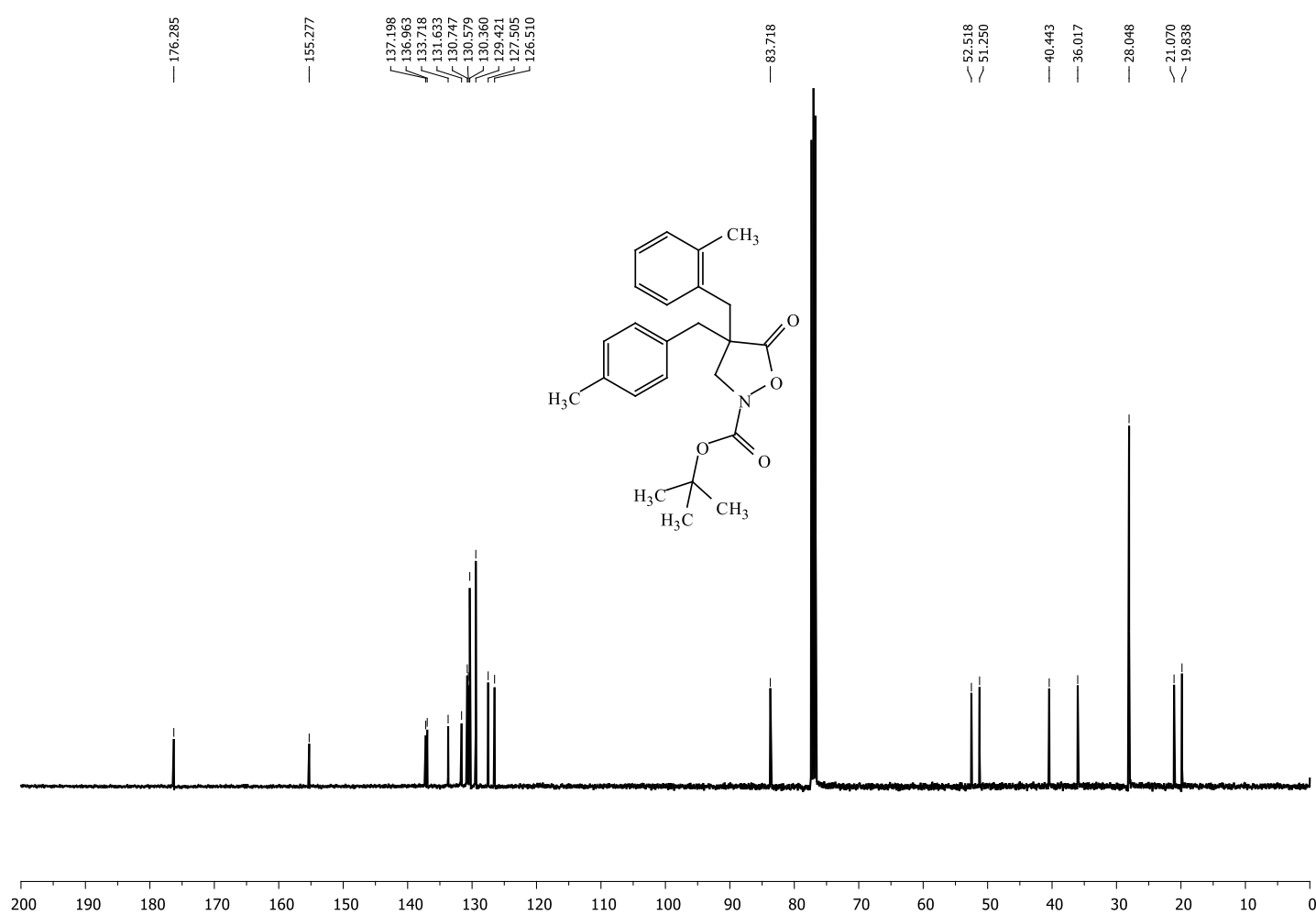

Page S58 of S104 
tert-Butyl 4-(4-bromobenzyl)-4-(4-chlorobenzyl)-5-oxoisoxazolidine-2-carboxylate (Boc-3e) ${ }^{1} \mathrm{H}$ NMR (400 MHz, $\mathrm{CDCl}_{3}$ )

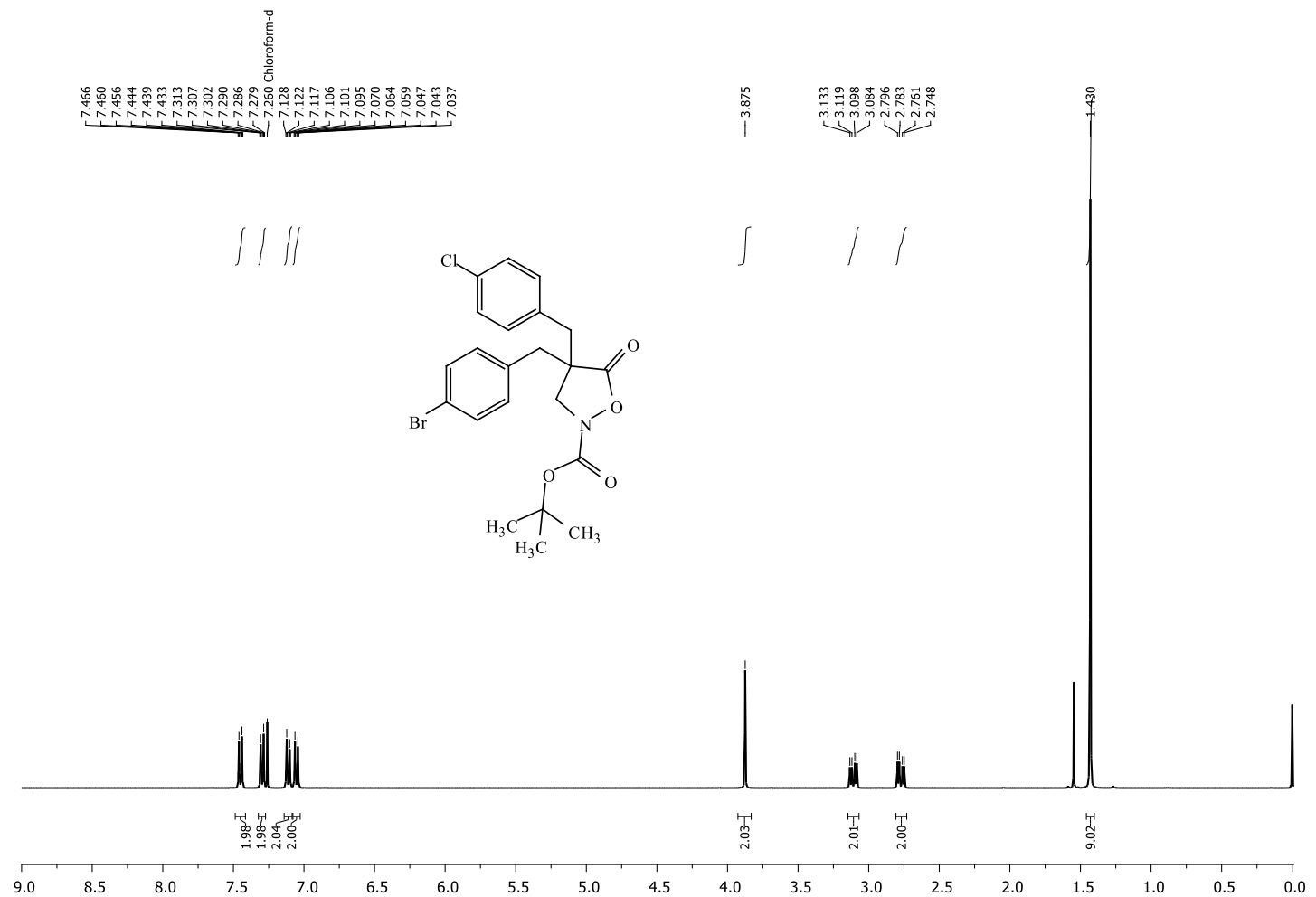

${ }^{13} \mathrm{C}$ NMR (101 MHz, $\left.\mathrm{CDCl}_{3}\right)$
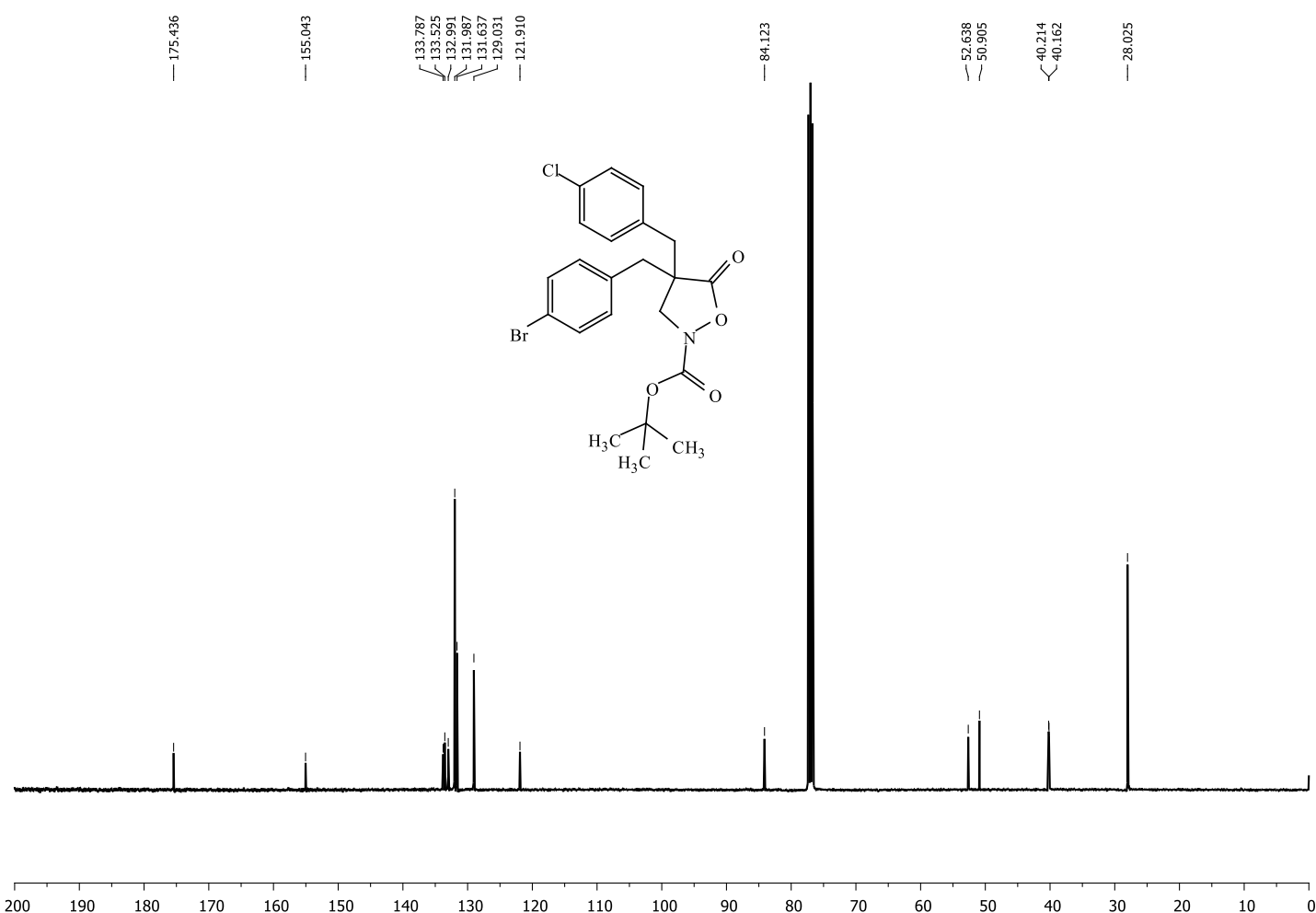

Page S59 of S104 
tert-Butyl 4-(cyclohexylmethyl)-4-(4-methylbenzyl)-5-oxoisoxazolidine-2-carboxylate (Boc-7b) ${ }^{1} \mathrm{H}$ NMR (400 MHz, $\left.\mathrm{CDCl}_{3}\right)$

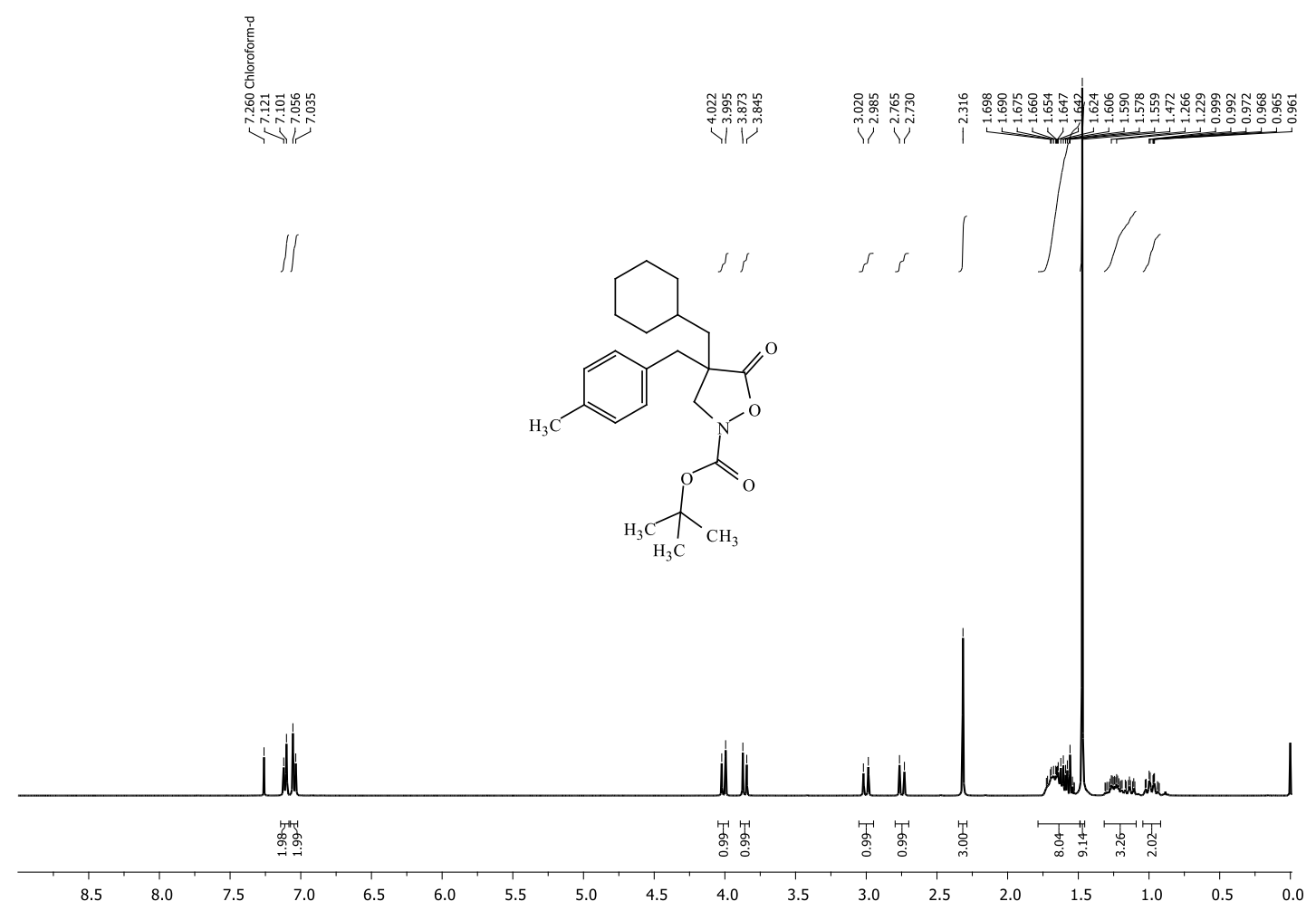

${ }^{13} \mathrm{C}$ NMR (101 MHz, $\left.\mathrm{CDCl}_{3}\right)$

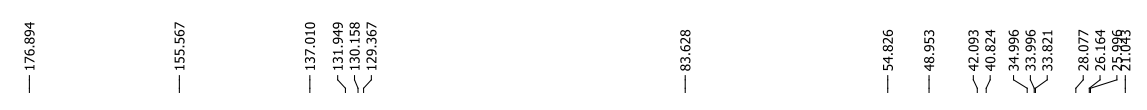

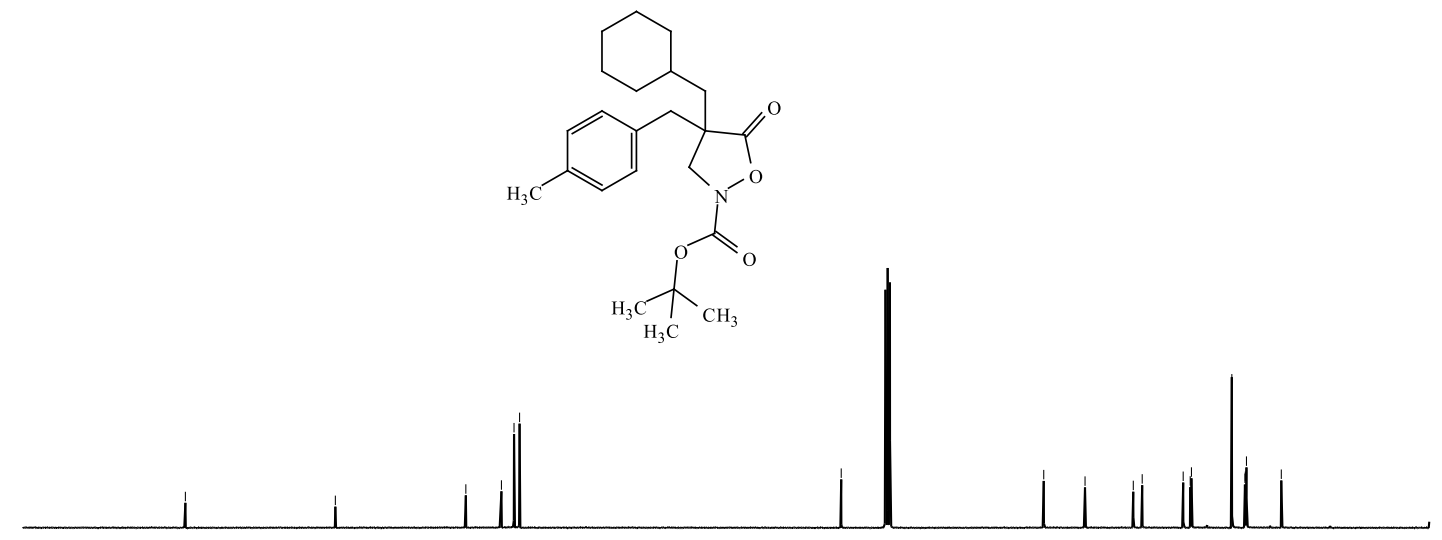

Page S60 of S104 
tert-Butyl 4-(cyclohexylmethyl)-4-(2-methylbenzyl)-5-oxoisoxazolidine-2-carboxylate (Boc-7c) ${ }^{1} \mathrm{H}$ NMR (400 MHz, $\mathrm{CDCl}_{3}$ )

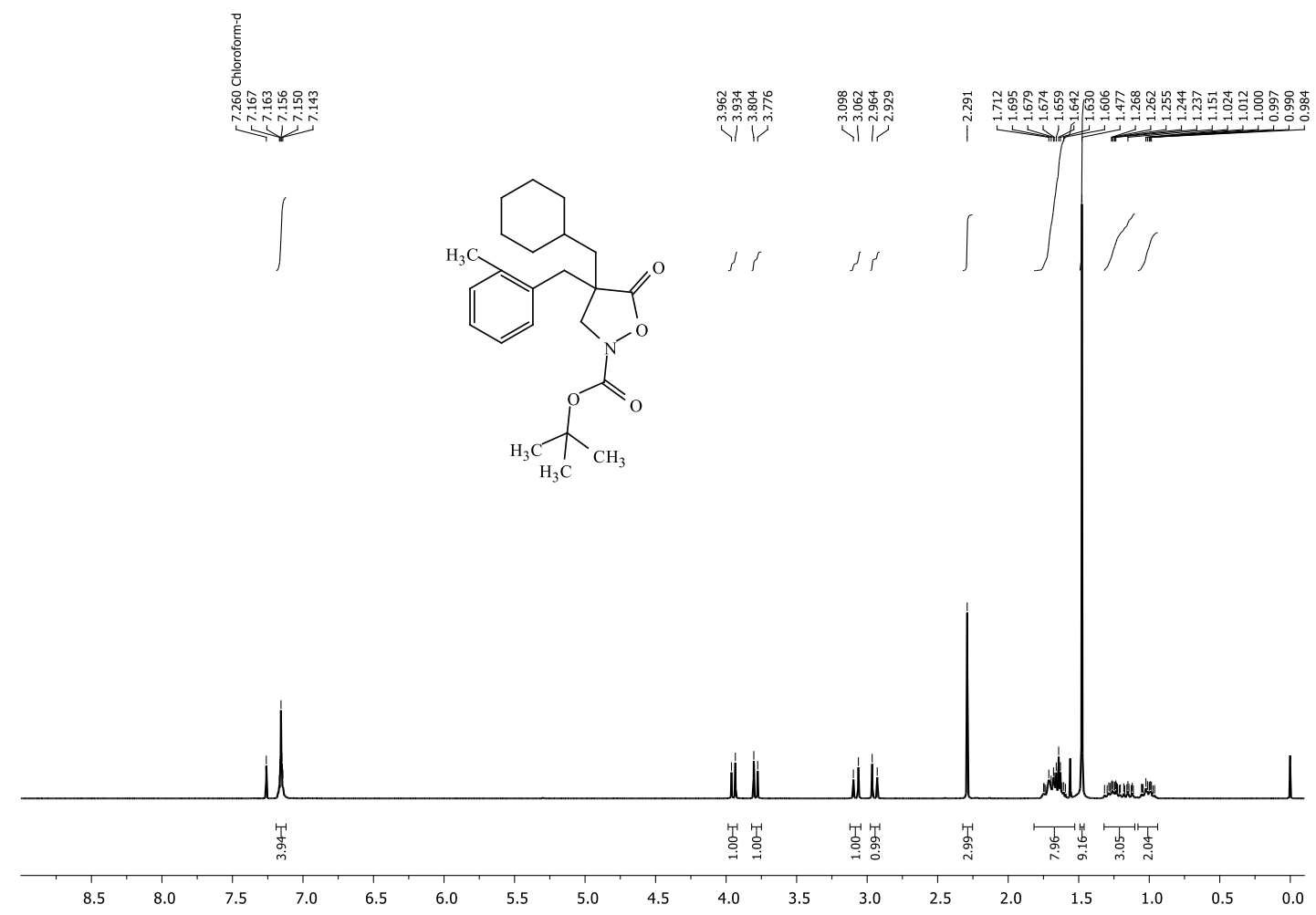

${ }^{13} \mathrm{C}$ NMR (101 MHz, $\left.\mathrm{CDCl}_{3}\right)$
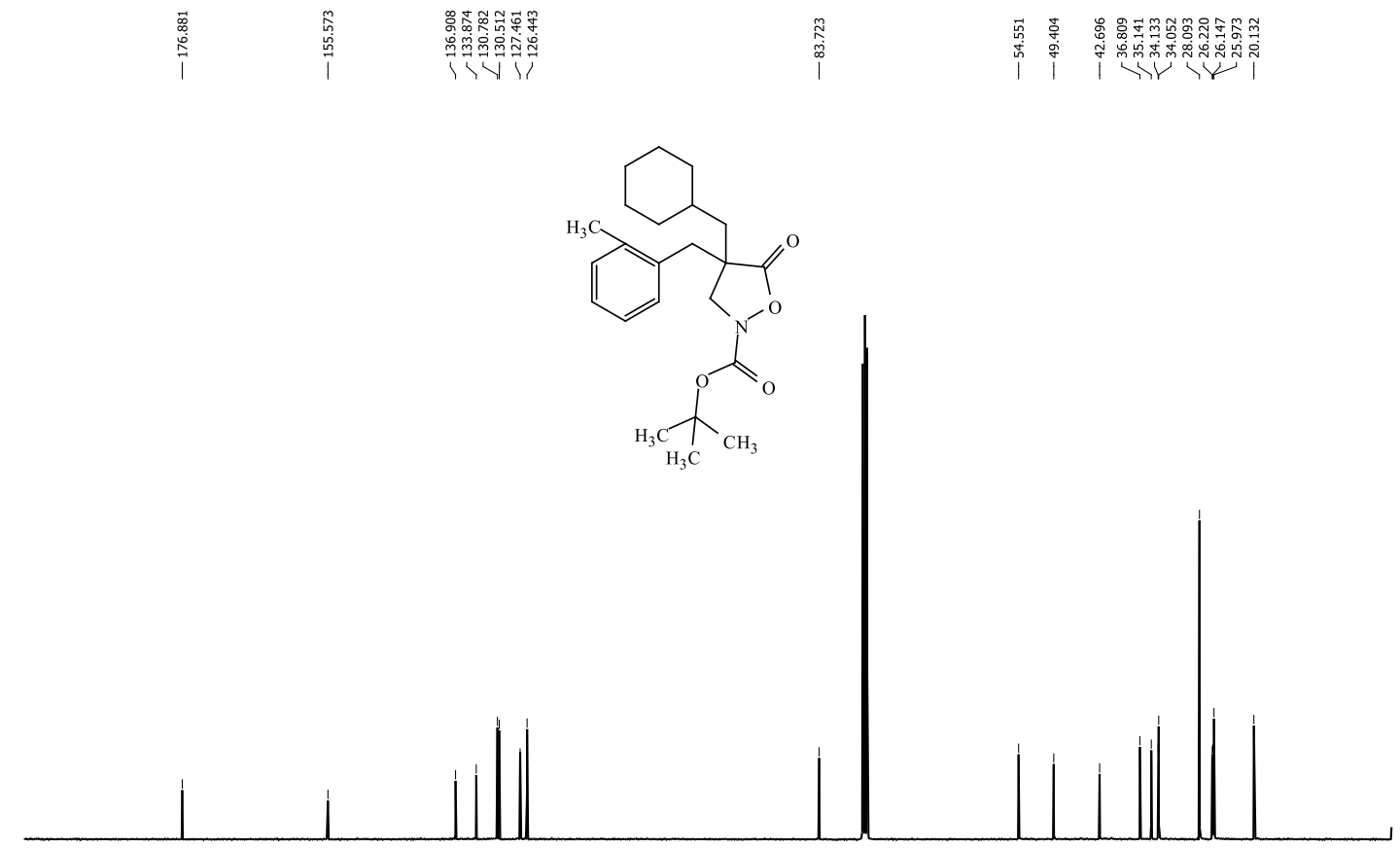

$\begin{array}{lllllllllll}200 & 190 & 180 & 170 & 160 & 150 & 140 & 130 & 120 & 110 & 100\end{array}$

Page S61 of S104 
tert-Butyl 4-([1,1'-biphenyl]-4-ylmethyl)-4-(cyclohexylmethyl)-5-oxoisoxazolidine-2-carboxylate (Boc-7d) ${ }^{1} \mathrm{H}$ NMR (400 MHz, $\left.\mathrm{CDCl}_{3}\right)$

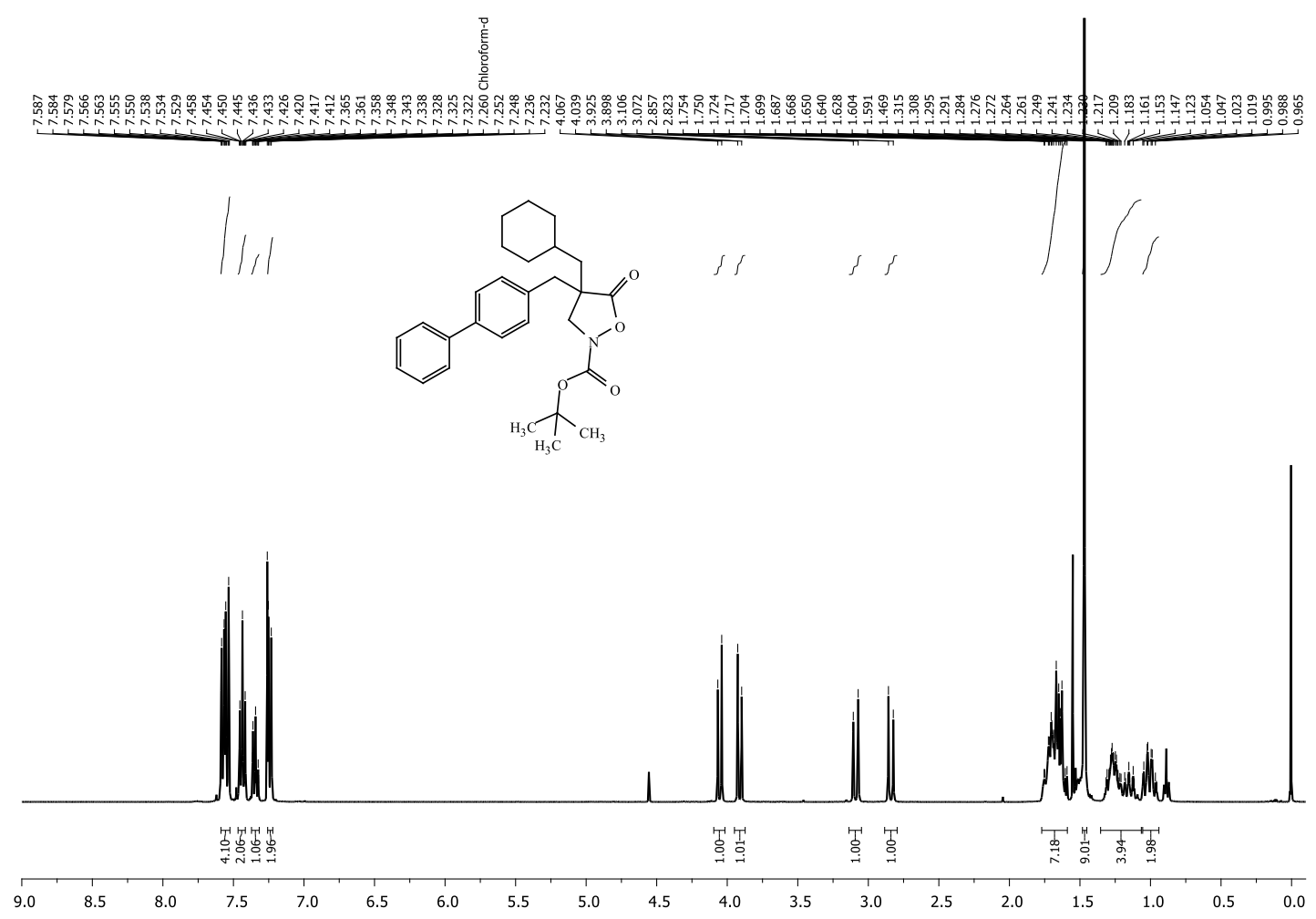

${ }^{13} \mathrm{C}$ NMR (101 MHz, $\left.\mathrm{CDCl}_{3}\right)$
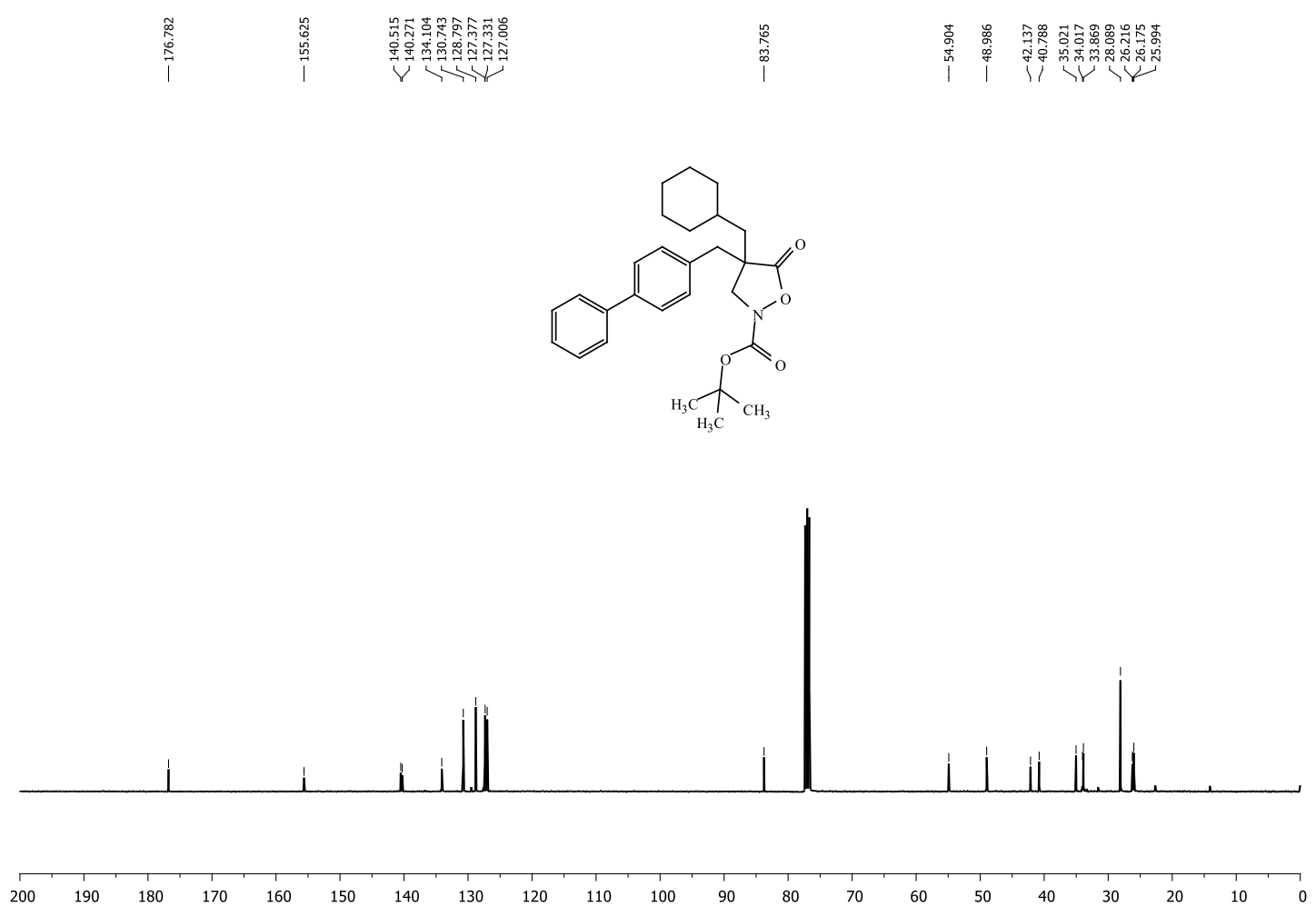
tert-Butyl 4-benzyl-5-oxo-4-((tetrahydro-2H-pyran-4-yl)methyl)isoxazolidine-2-carboxylate (Boc-7e) ${ }^{1} \mathrm{H}$ NMR (400 MHz, $\left.\mathrm{CDCl}_{3}\right)$

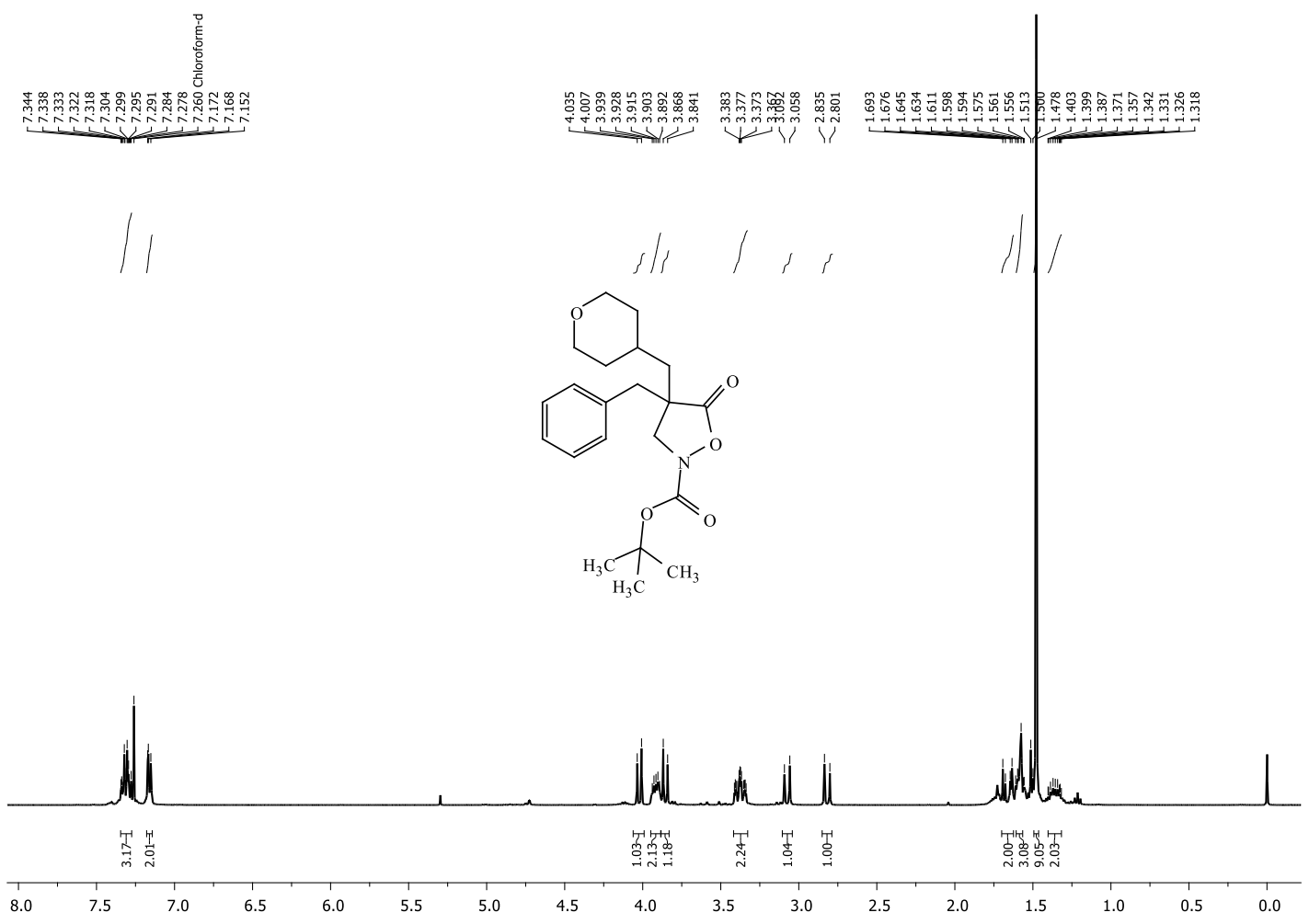

${ }^{13} \mathrm{C}$ NMR (101 MHz, $\left.\mathrm{CDCl}_{3}\right)$

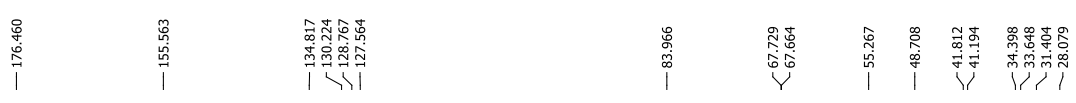

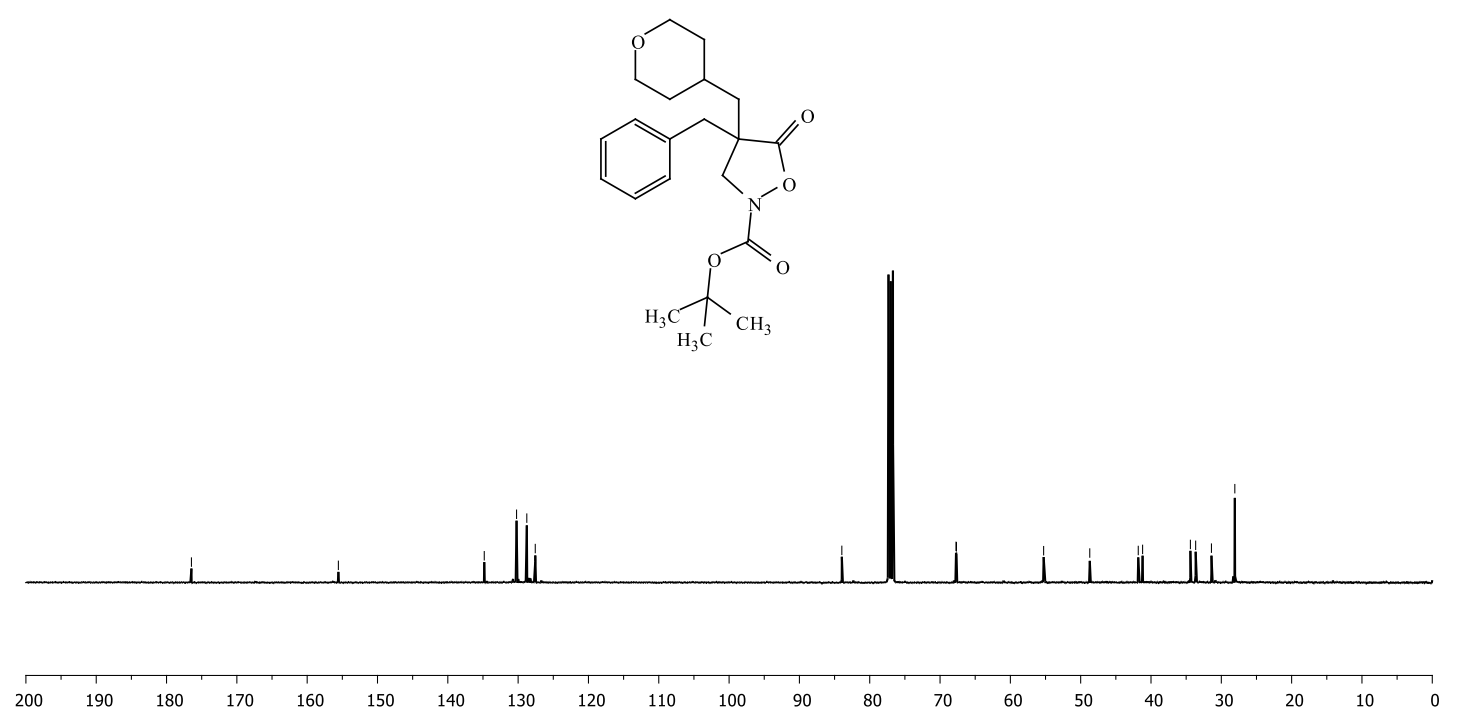

Page S63 of S104 
tert-Butyl 4-benzyl-4-(4-nitrobenzyl)-5-oxoisoxazolidine-2-carboxylate (Boc-7f)

${ }^{1} \mathrm{H}$ NMR (400 MHz, $\left.\mathrm{CDCl}_{3}\right)$
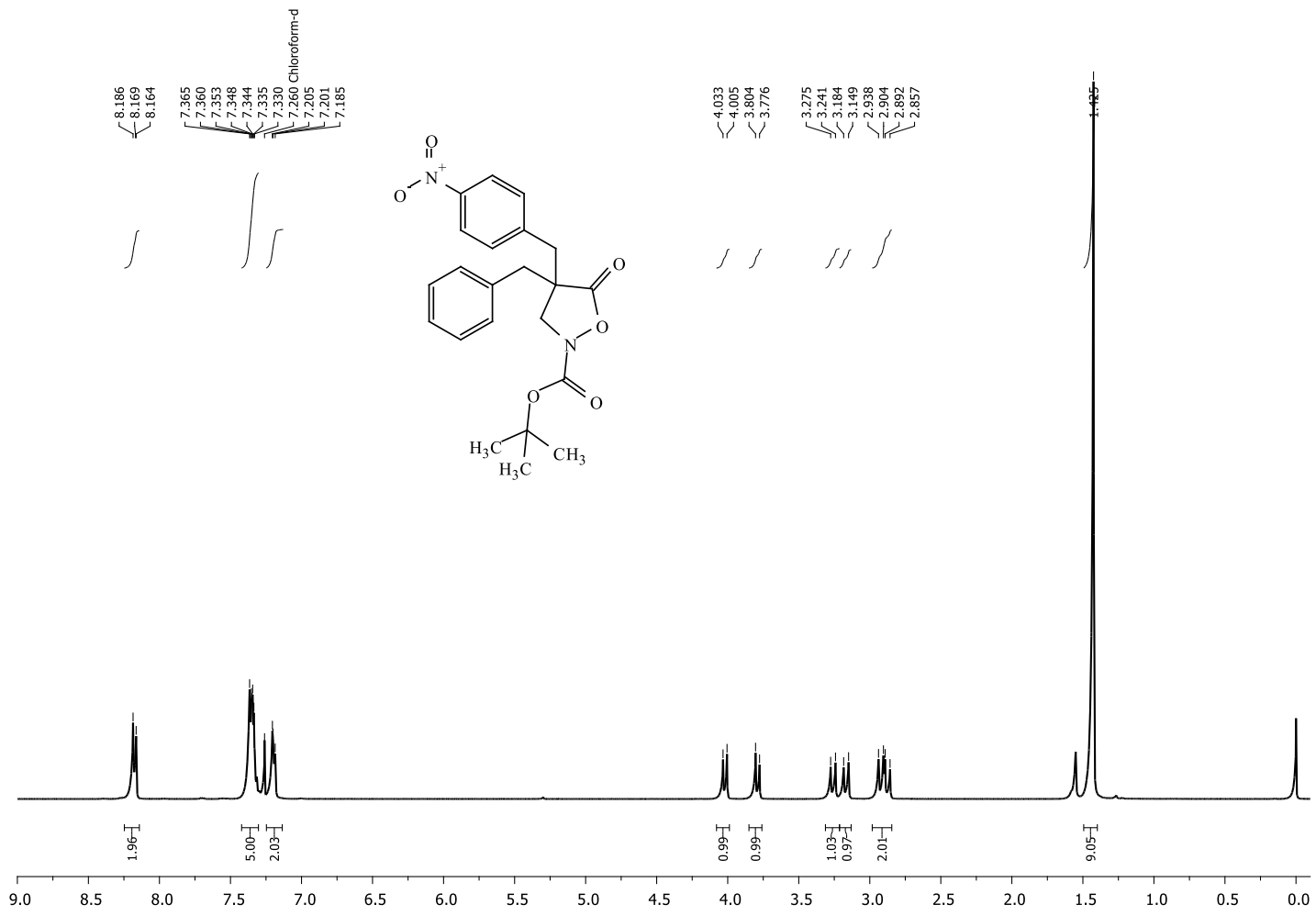

${ }^{13} \mathrm{C}$ NMR (101 MHz, $\left.\mathrm{CDCl}_{3}\right)$

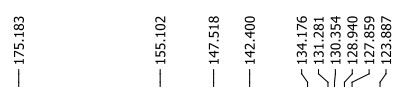
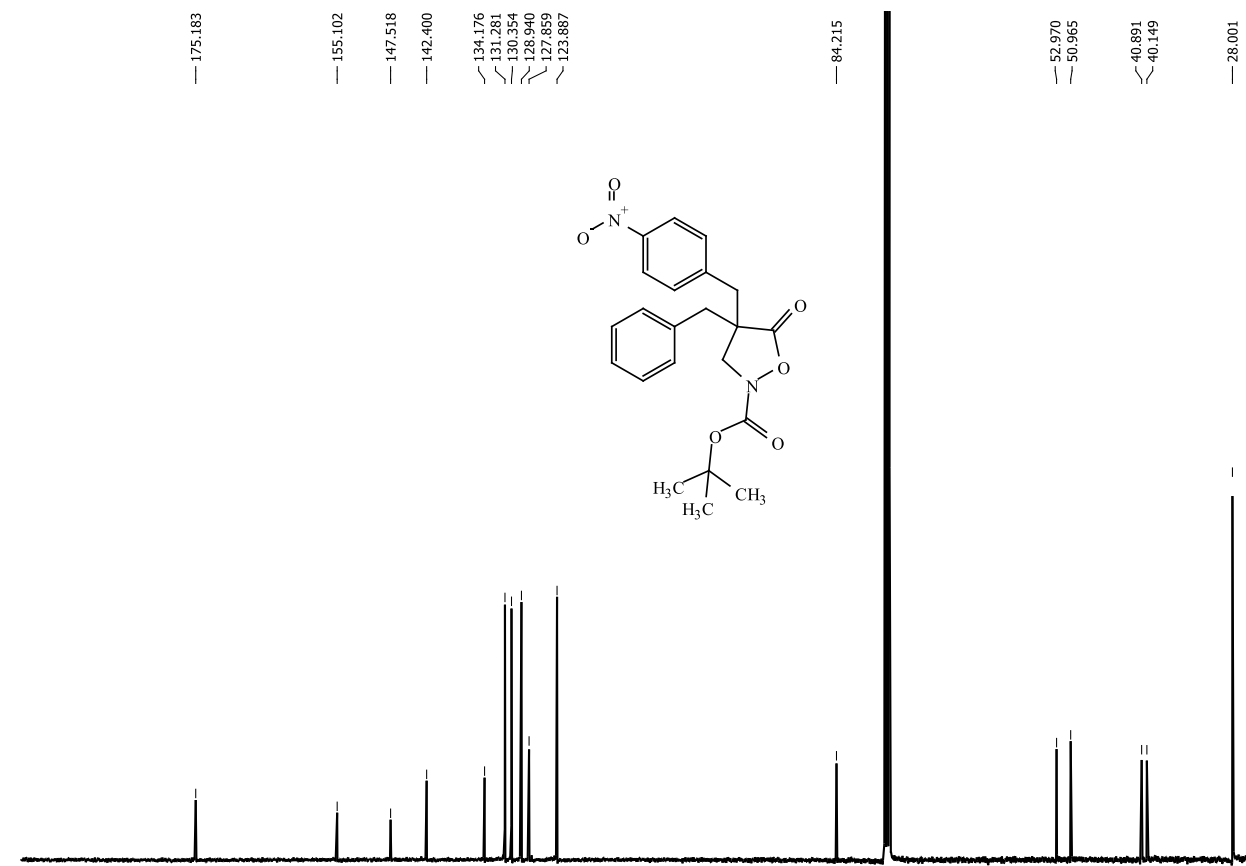

$$
200
$$

200
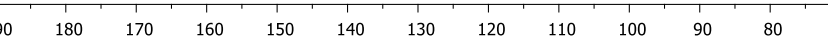

Page S64 of S104 


\section{4,4-Bis(4-methylbenzyl)isoxazolidin-5-one (1b)}

${ }^{1} \mathrm{H}$ NMR (400 MHz, $\mathrm{CDCl}_{3}$ )

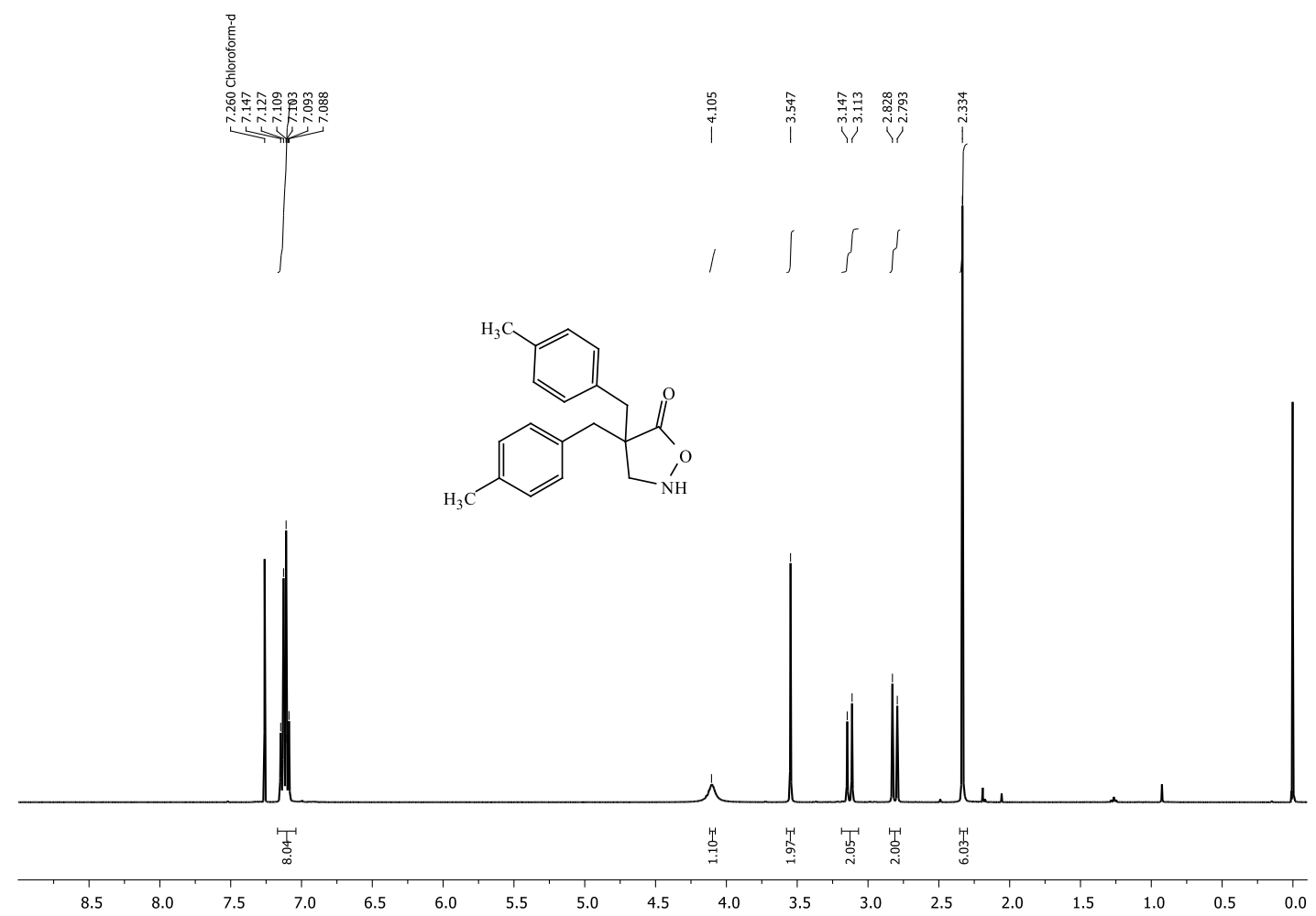

${ }^{13} \mathrm{C}$ NMR (101 MHz, $\left.\mathrm{CDCl}_{3}\right)$

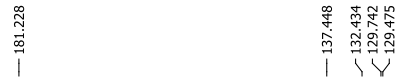

|V

崫
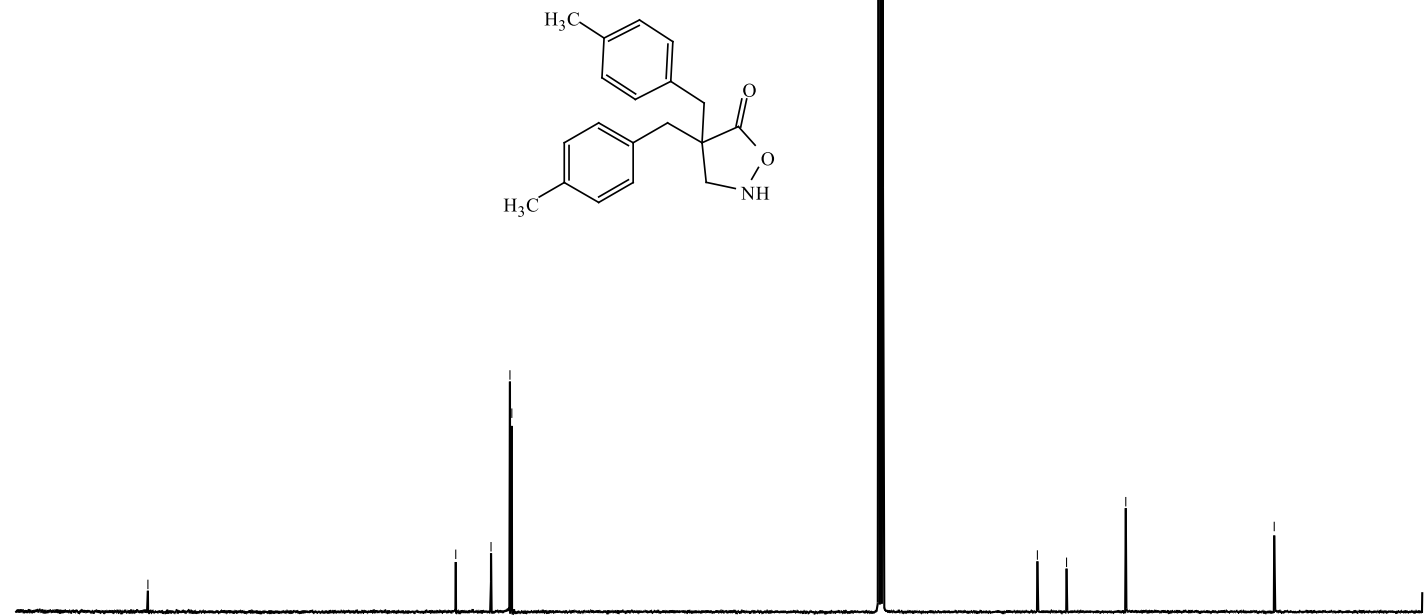

Page S65 of S104 


\section{4,4-Bis(2-methylbenzyl)isoxazolidin-5-one (1c)}

${ }^{1} \mathrm{H}$ NMR (400 MHz, $\mathrm{CDCl}_{3}$ )

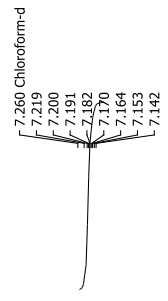<smiles>Cc1ccccc1CC1(Cc2ccccc2C)CNOC1=O</smiles>

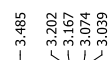

|ं लंखं
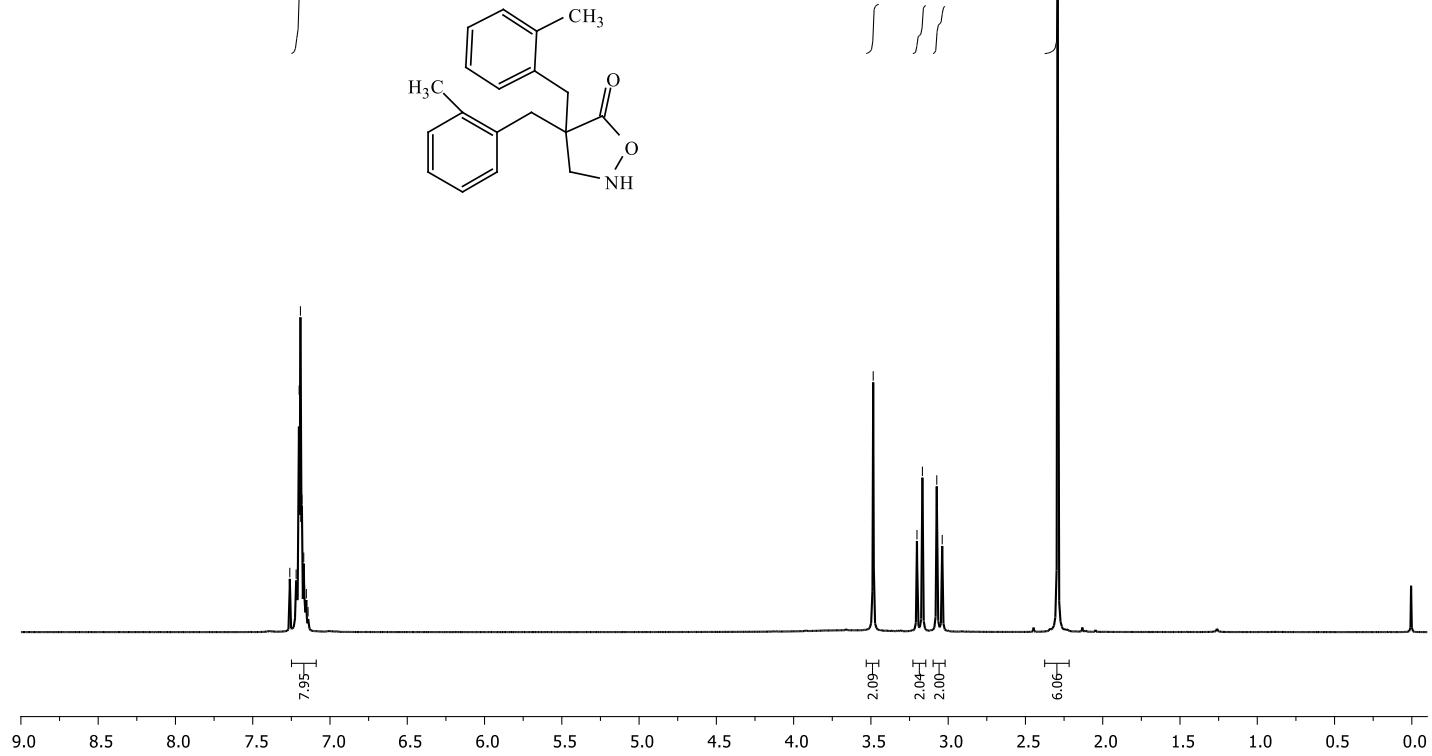

${ }^{13} \mathrm{C}$ NMR (101 MHz, $\left.\mathrm{CDCl}_{3}\right)$
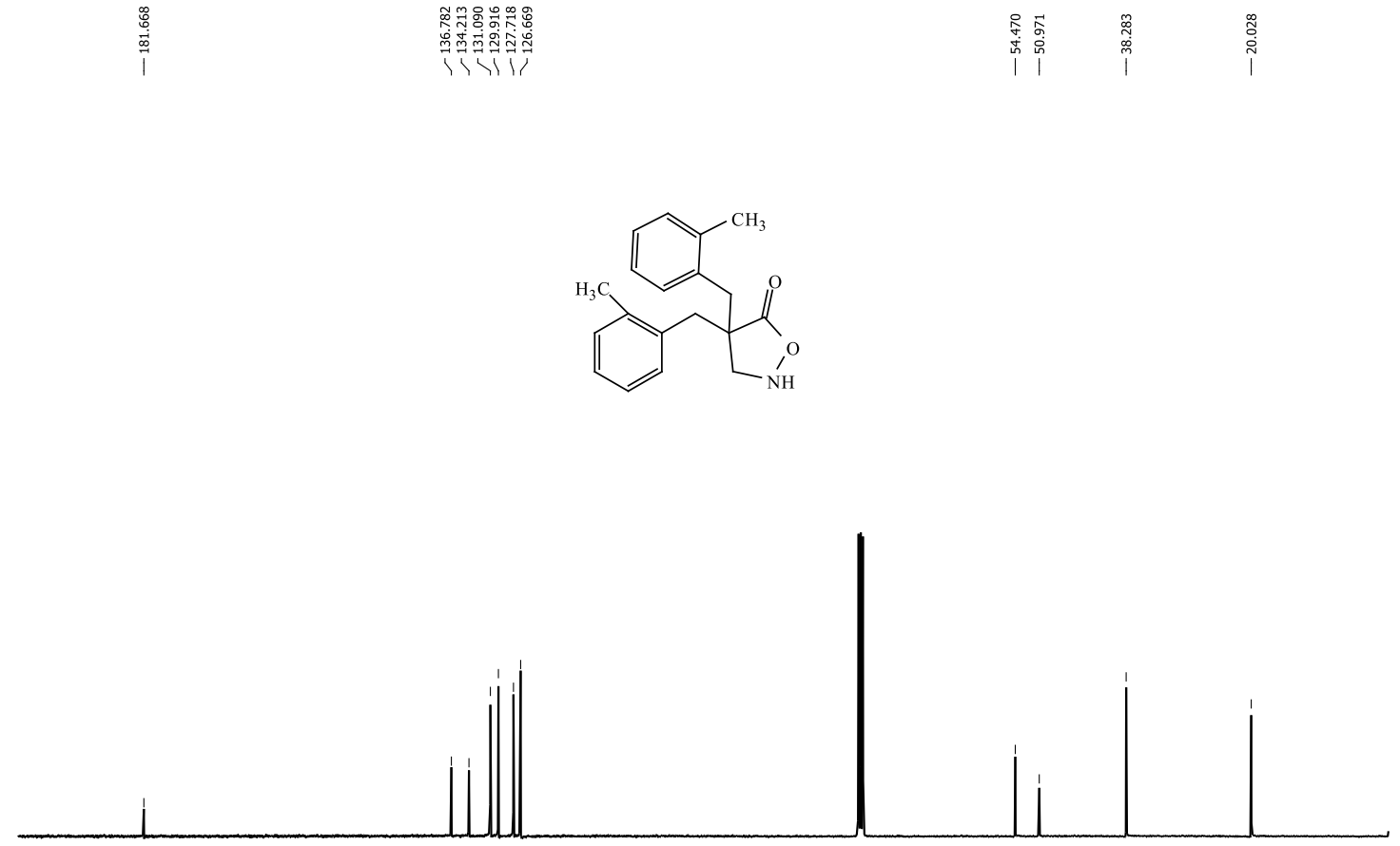

200 


\section{4,4-Bis([1,1'-biphenyl]-4-ylmethyl)isoxazolidin-5-one (1d)}

${ }^{1} \mathrm{H}$ NMR (400 MHz, $\mathrm{CDCl}_{3}$ )

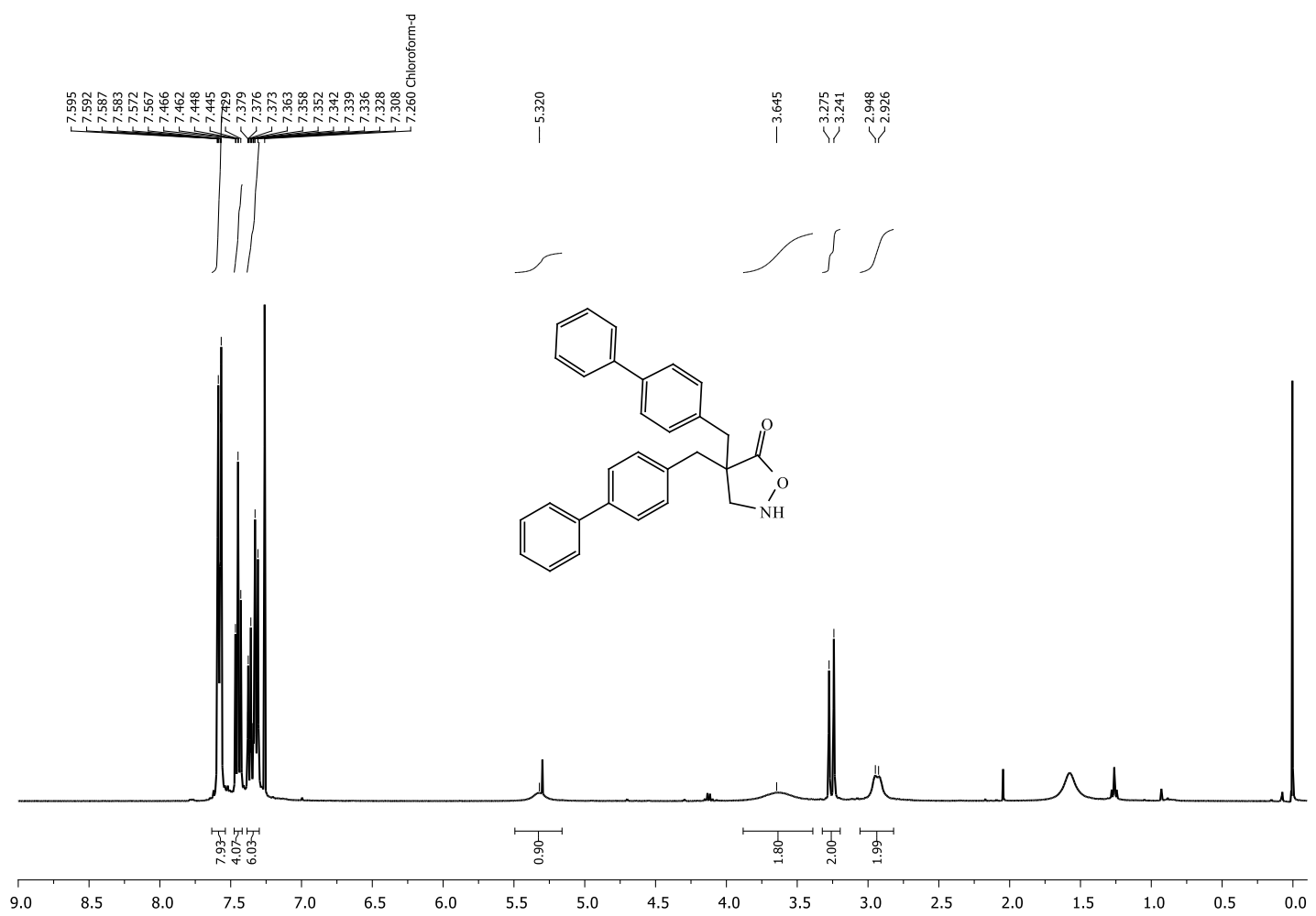

${ }^{13} \mathrm{C}$ NMR (101 MHz, $\left.\mathrm{CDCl}_{3}\right)$

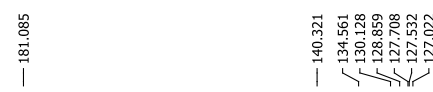

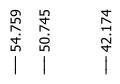
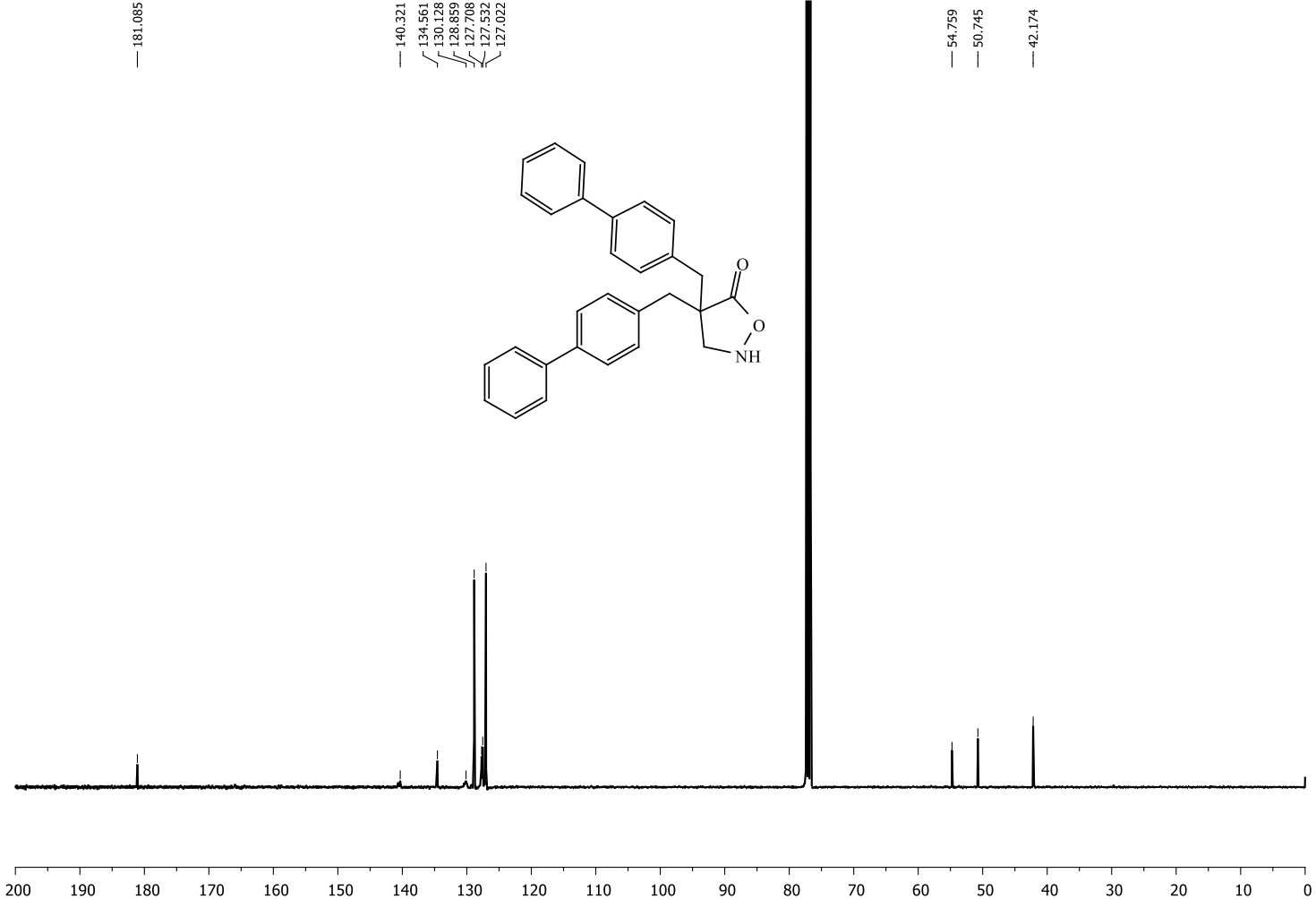

Page S67 of S104 


\section{4,4-Bis(4-(tert-butyl)benzyl)isoxazolidin-5-one (1e)}

${ }^{1} \mathrm{H}$ NMR (400 MHz, $\left.\mathrm{CDCl}_{3}\right)$

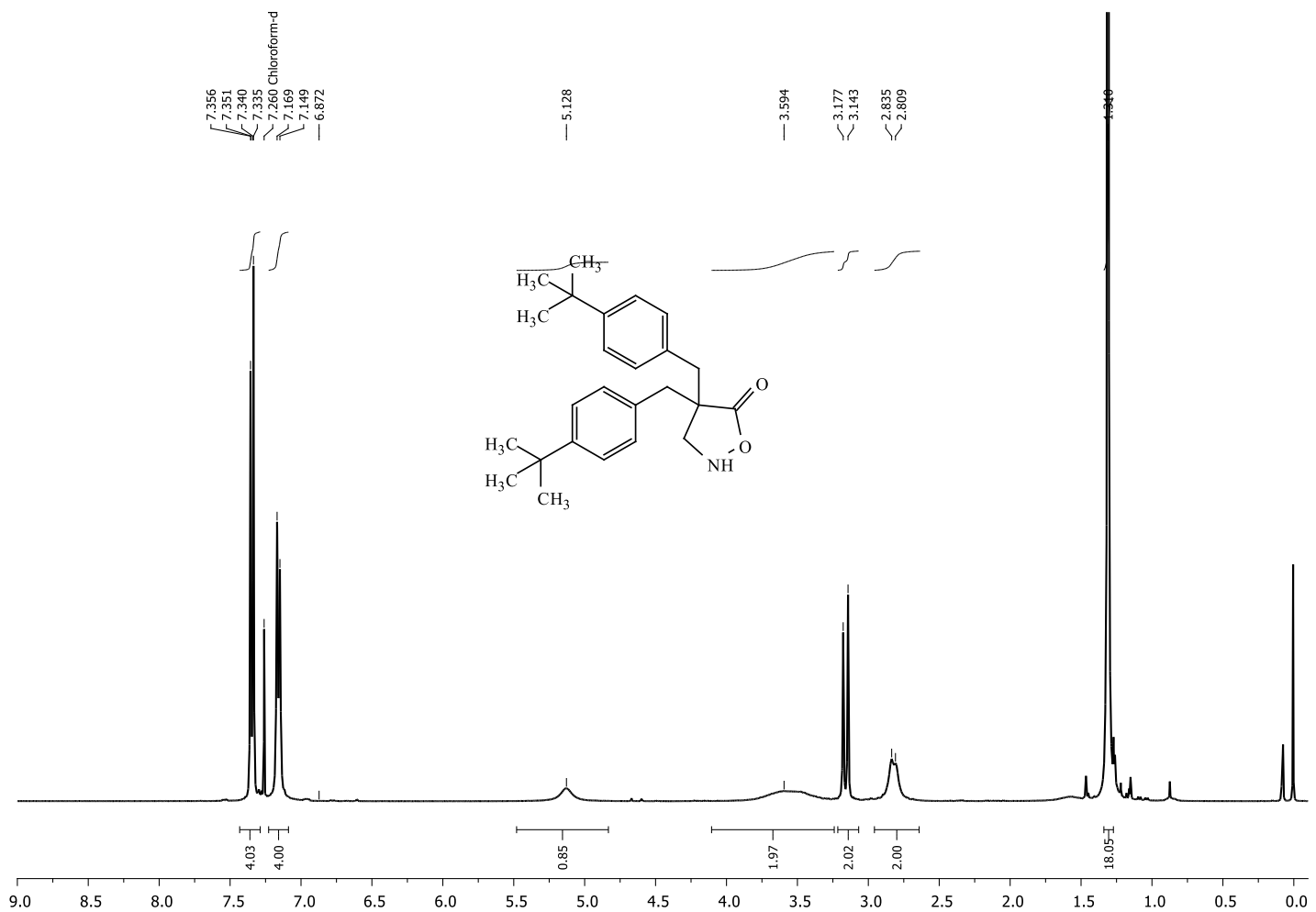

${ }^{13} \mathrm{C}$ NMR (101 MHz, $\left.\mathrm{CDCl}_{3}\right)$
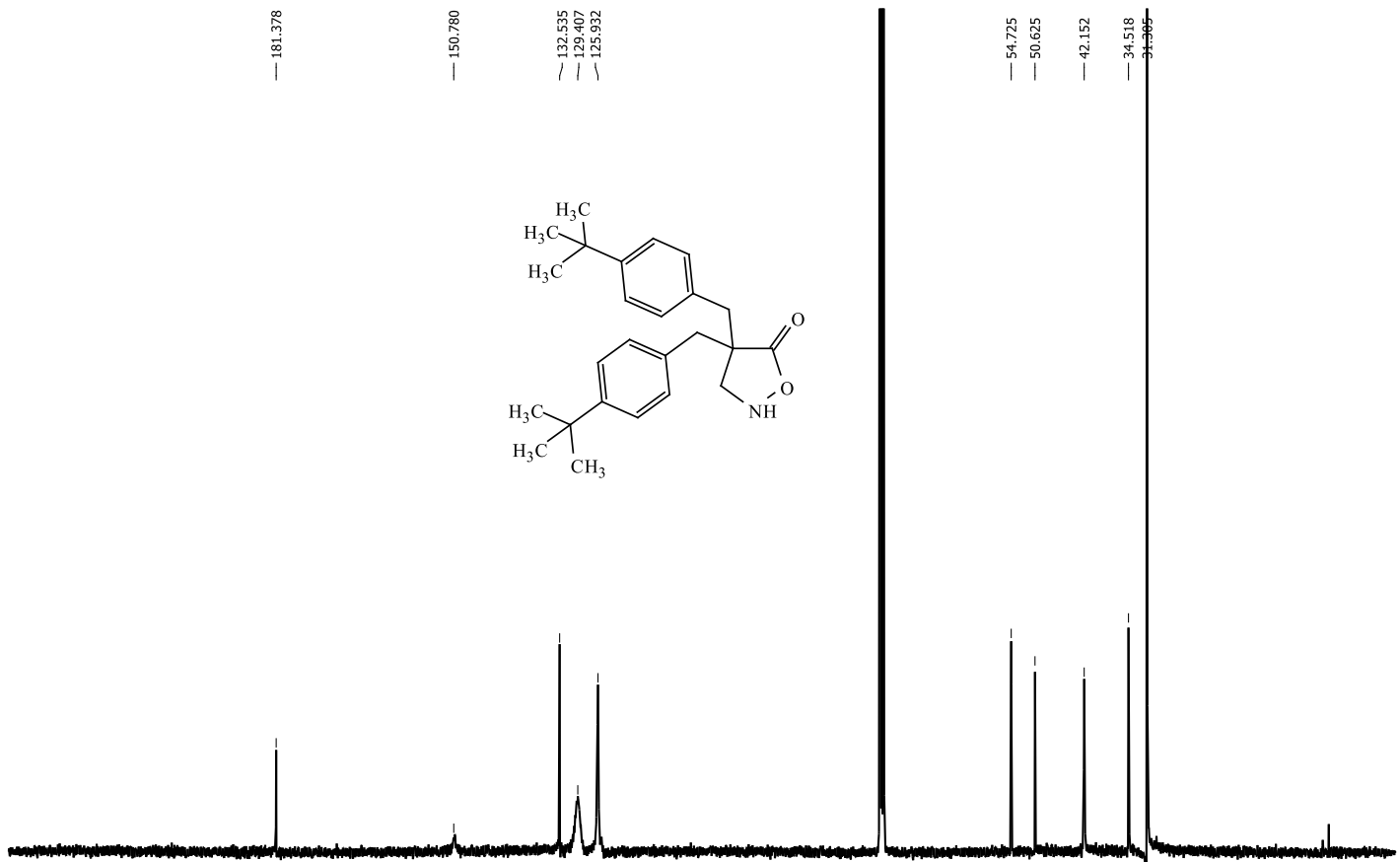

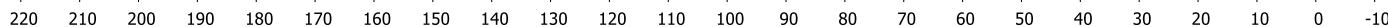




\section{4,4-Bis(4-bromobenzyl)isoxazolidin-5-one (1f)}

${ }^{1} \mathrm{H}$ NMR (400 MHz, $\mathrm{CDCl}_{3}$ )

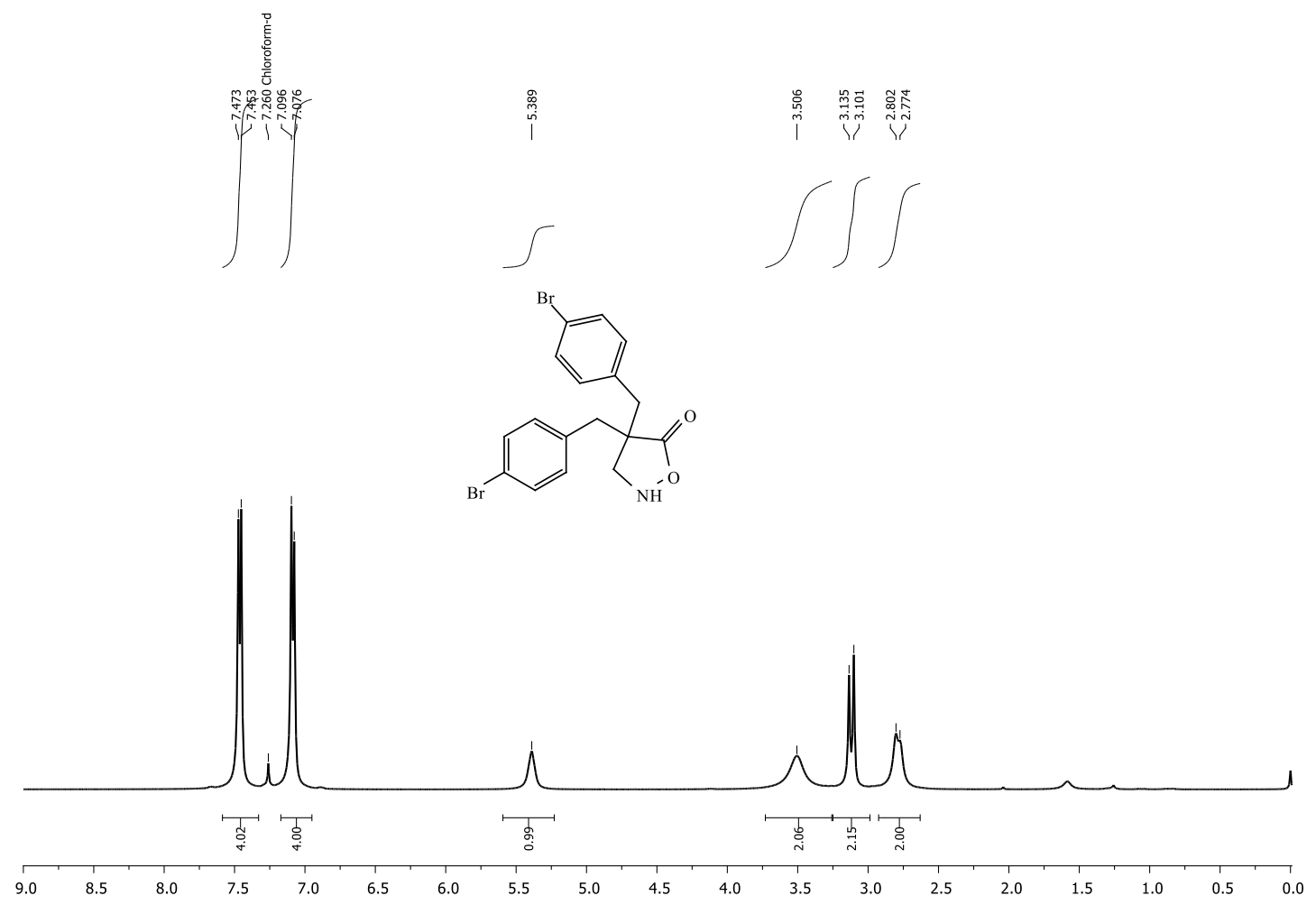

${ }^{13} \mathrm{C}$ NMR (101 MHz, $\left.\mathrm{CDCl}_{3}\right)$

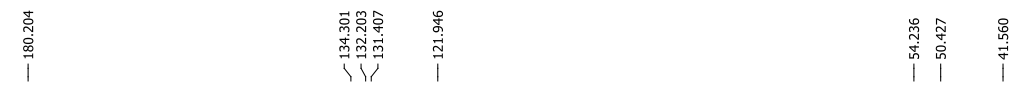

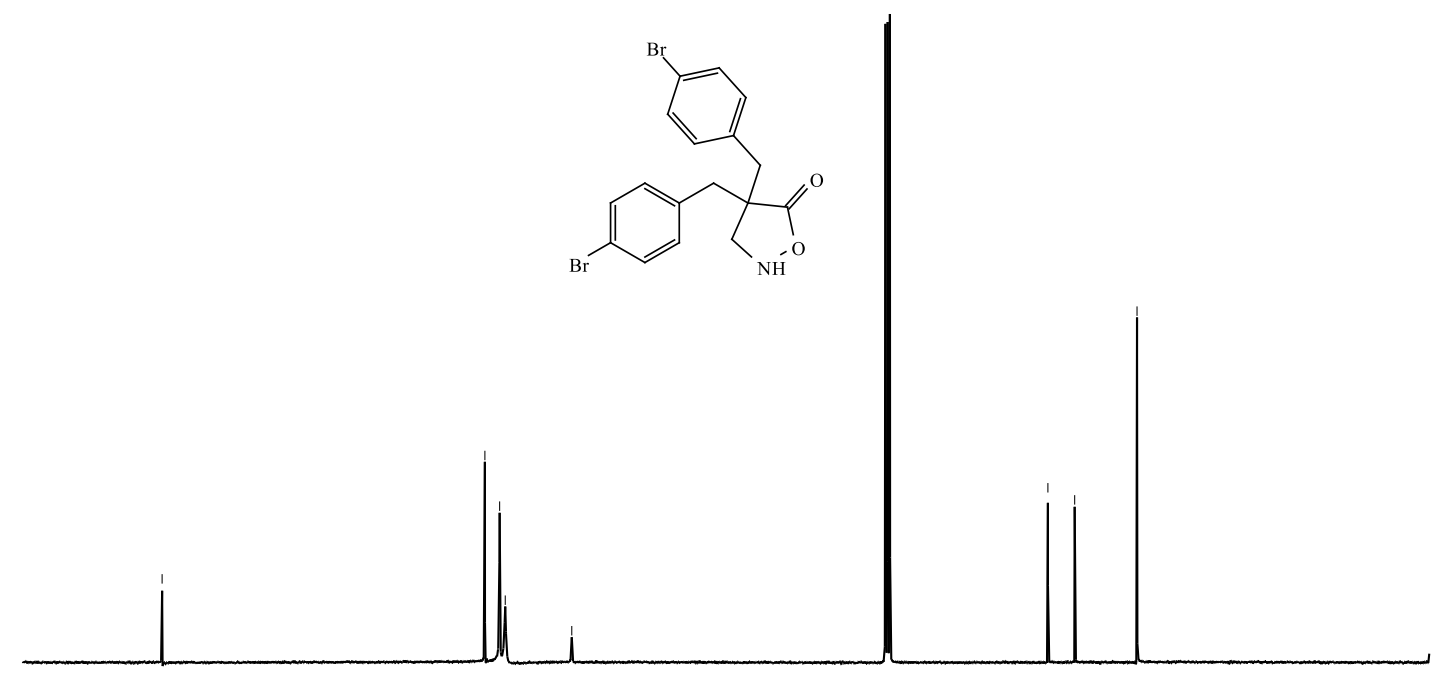

Page S69 of S104 
4,4-Bis(2-chlorobenzyl)isoxazolidin-5-one (1g)

${ }^{1} \mathrm{H}$ NMR (400 MHz, $\mathrm{CDCl}_{3}$ )
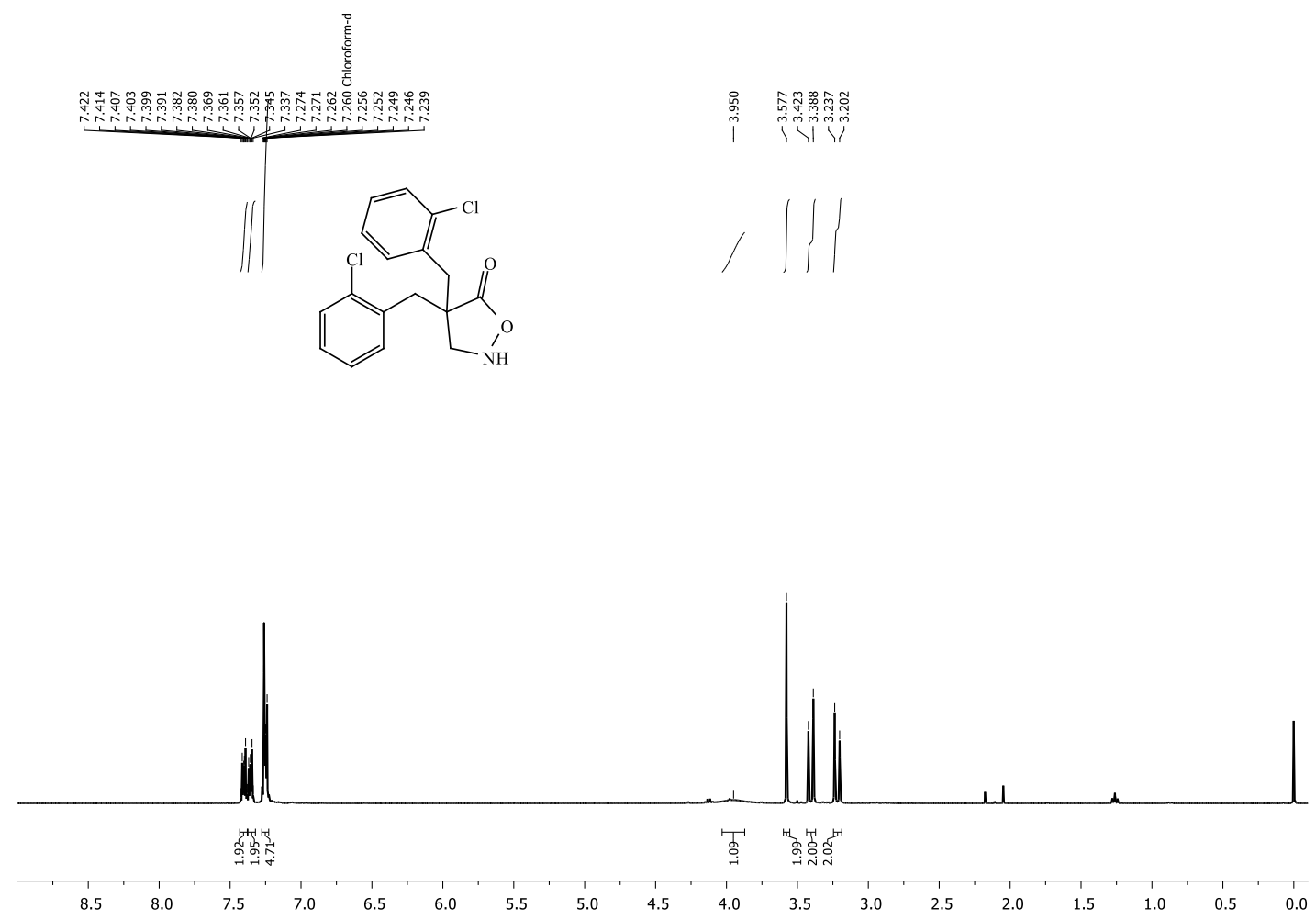

${ }^{13} \mathrm{C}$ NMR (101 MHz, $\left.\mathrm{CDCl}_{3}\right)$

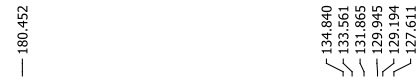

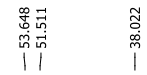
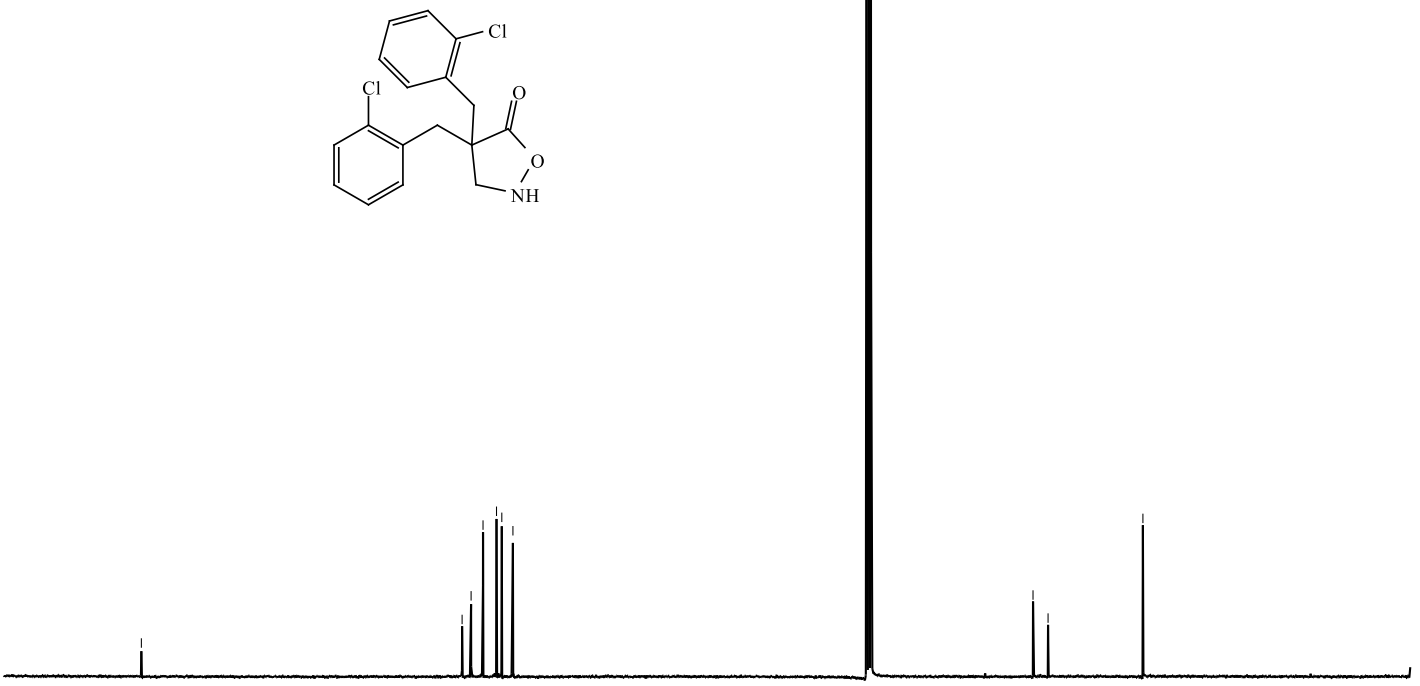

Page S70 of S104 
4-Benzyl-4-(2-methylbenzyl)isoxazolidin-5-one (3c)

${ }^{1} \mathrm{H}$ NMR (400 $\left.\mathrm{MHz}, \mathrm{CDCl}_{3}\right)$

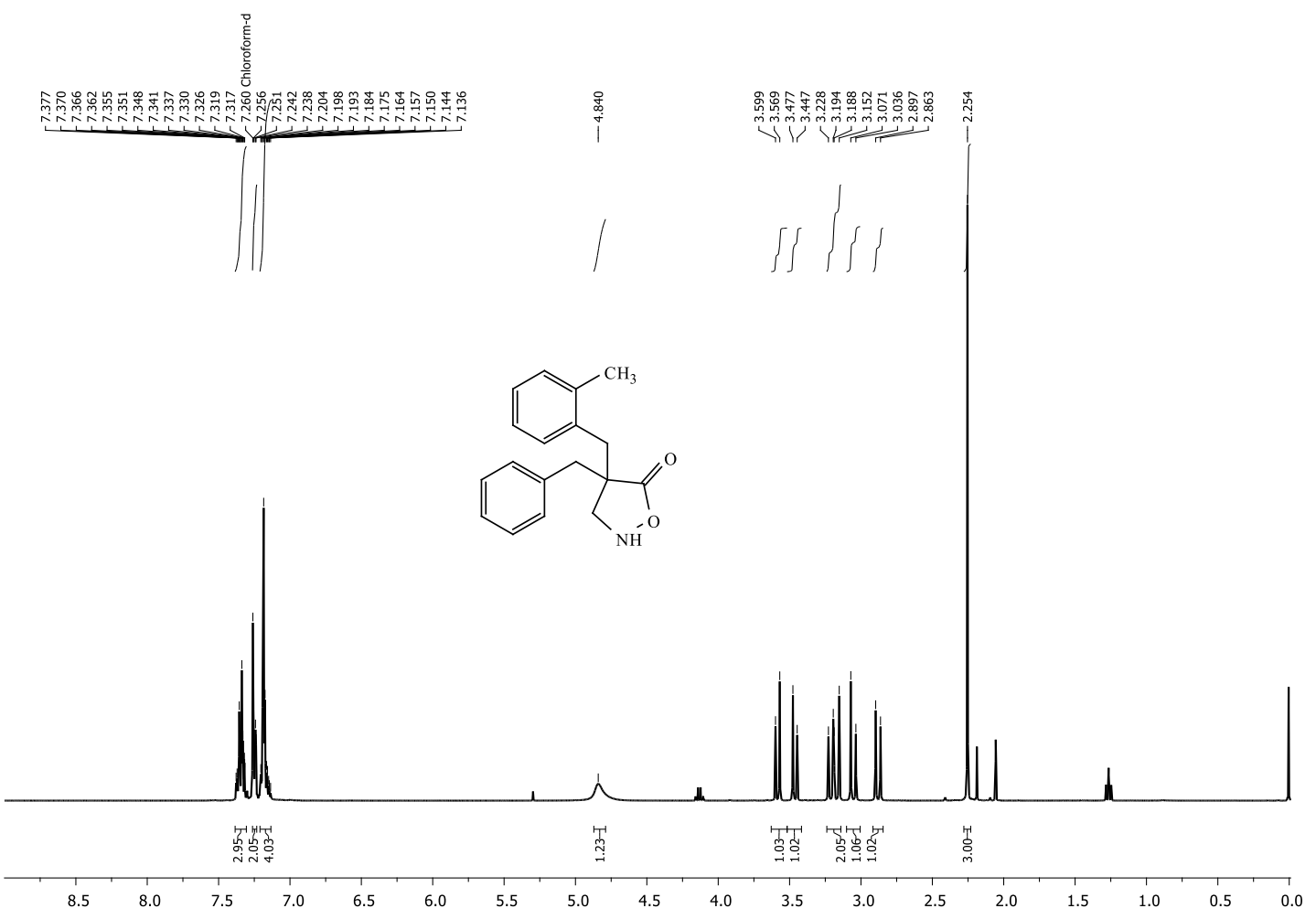

${ }^{13} \mathrm{C}$ NMR (101 MHz, $\left.\mathrm{CDCl}_{3}\right)$
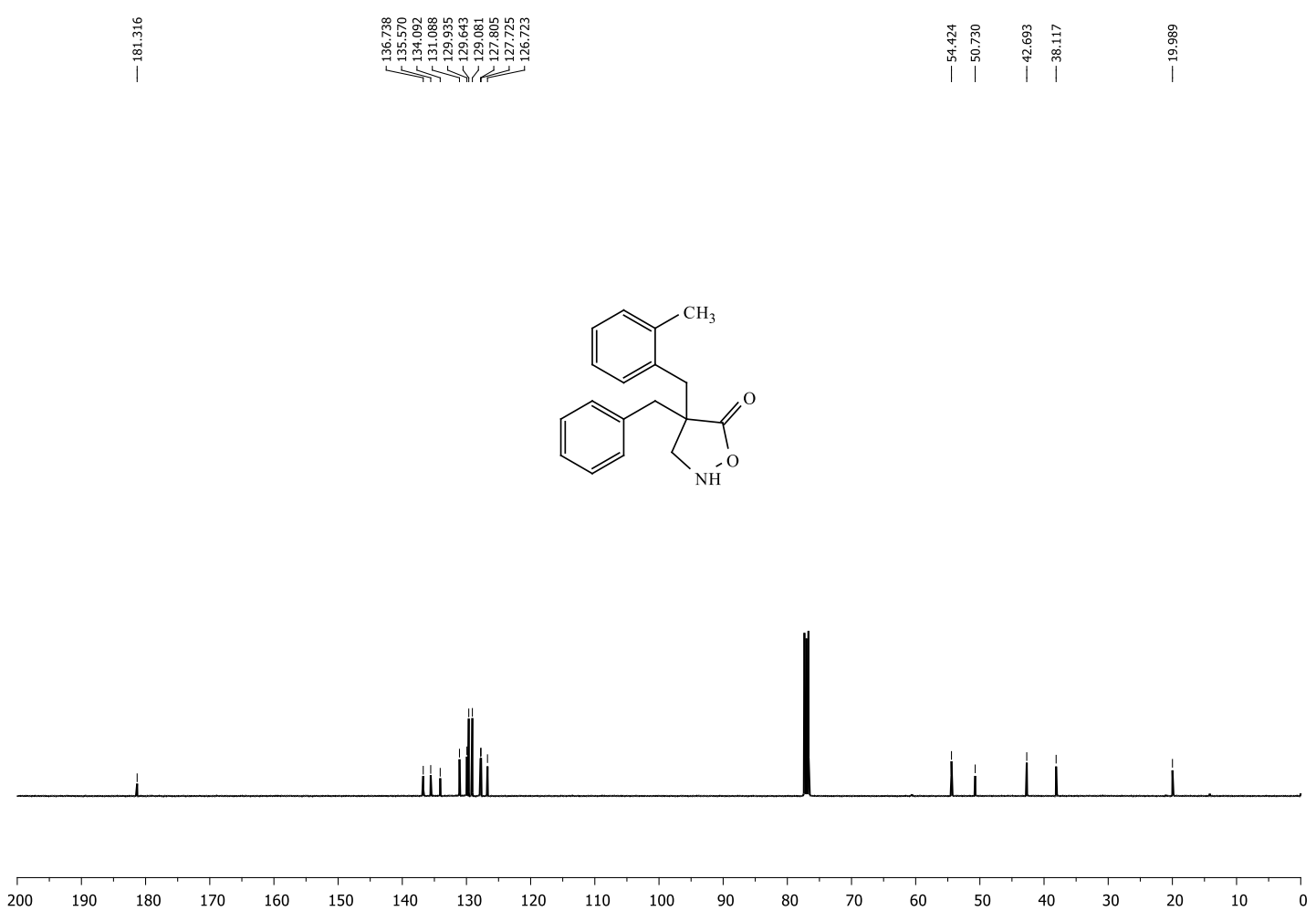
4-(2-Methylbenzyl)-4-(4-methylbenzyl)isoxazolidin-5-one (3d)

${ }^{1} \mathrm{H}$ NMR (400 MHz, $\left.\mathrm{CDCl}_{3}\right)$

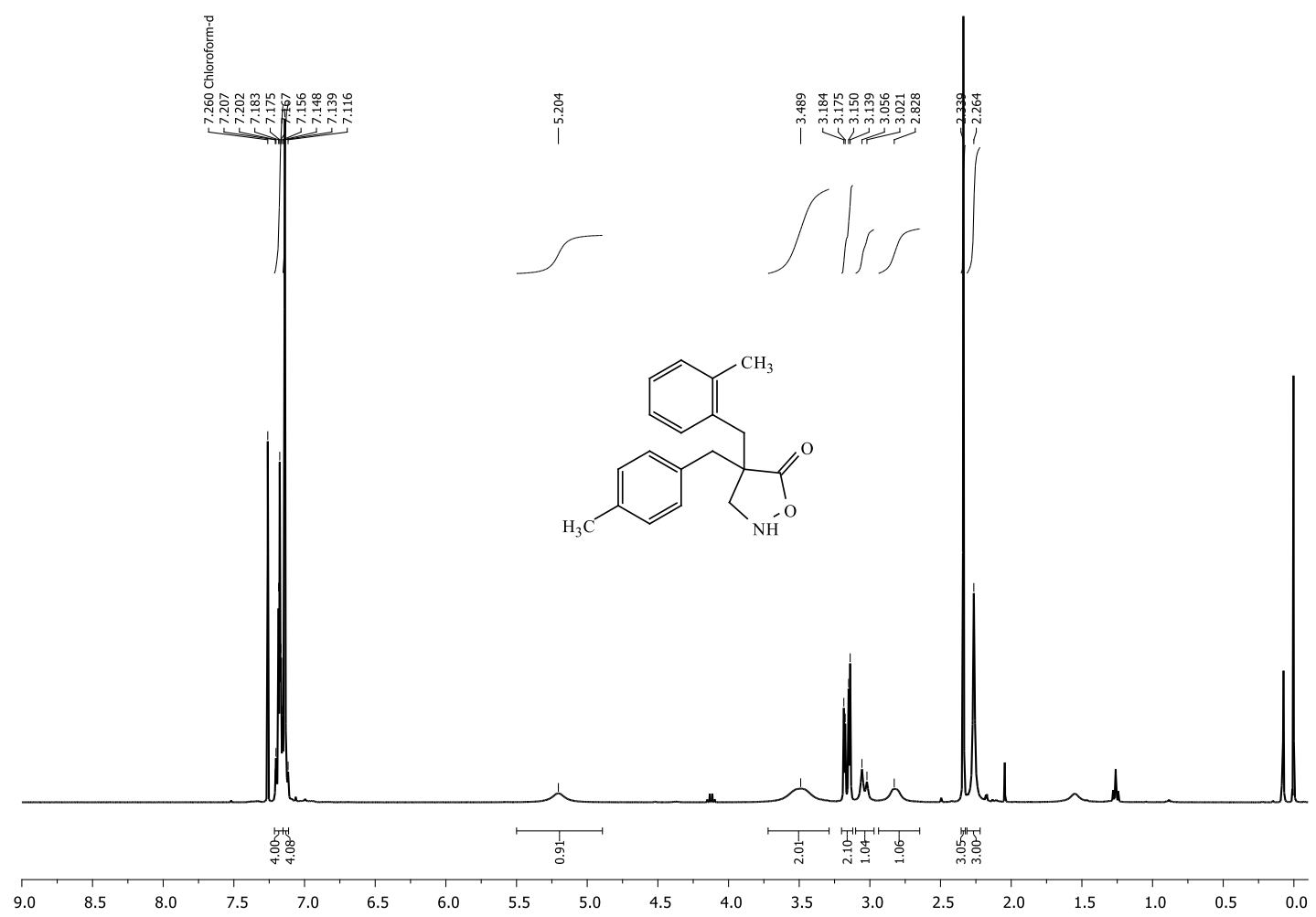

${ }^{13} \mathrm{C}$ NMR (101 MHz, $\left.\mathrm{CDCl}_{3}\right)$
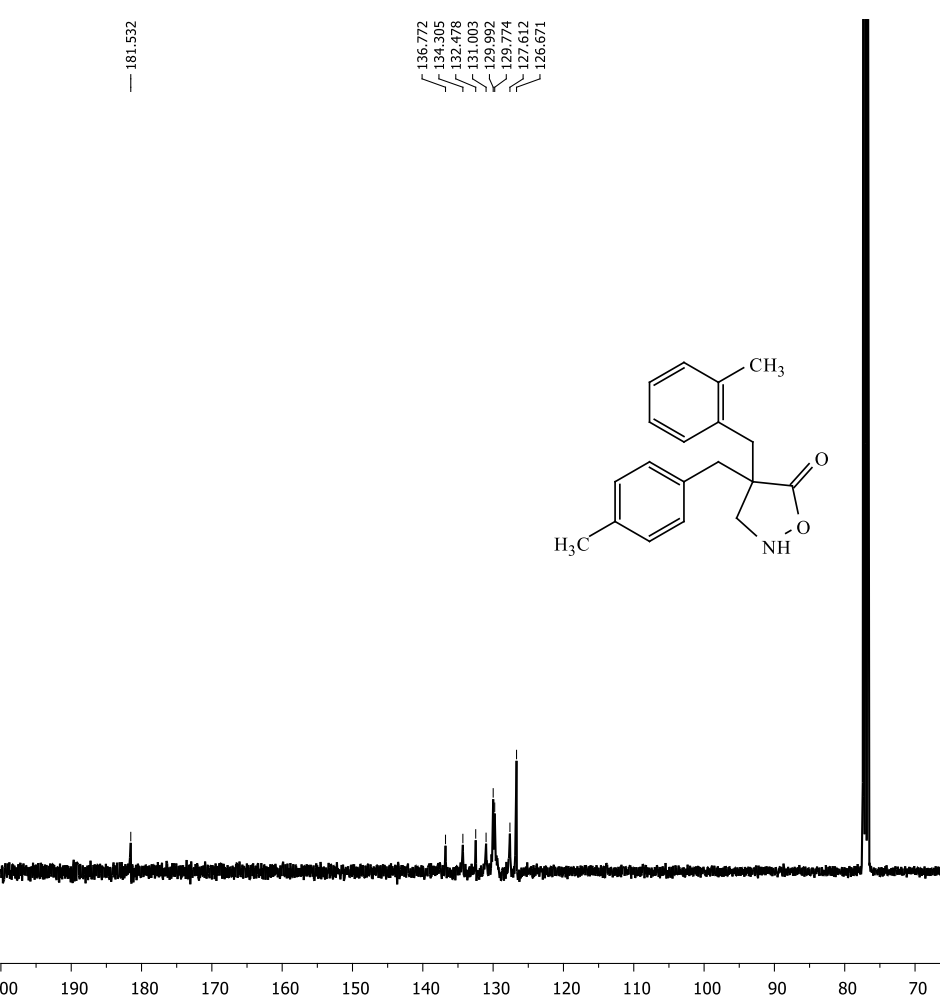

Page S72 of S104 
4-(4-Bromobenzyl)-4-(4-chlorobenzyl)isoxazolidin-5-one (3e)

${ }^{1} \mathrm{H}$ NMR (400 MHz, $\left.\mathrm{CDCl}_{3}\right)$
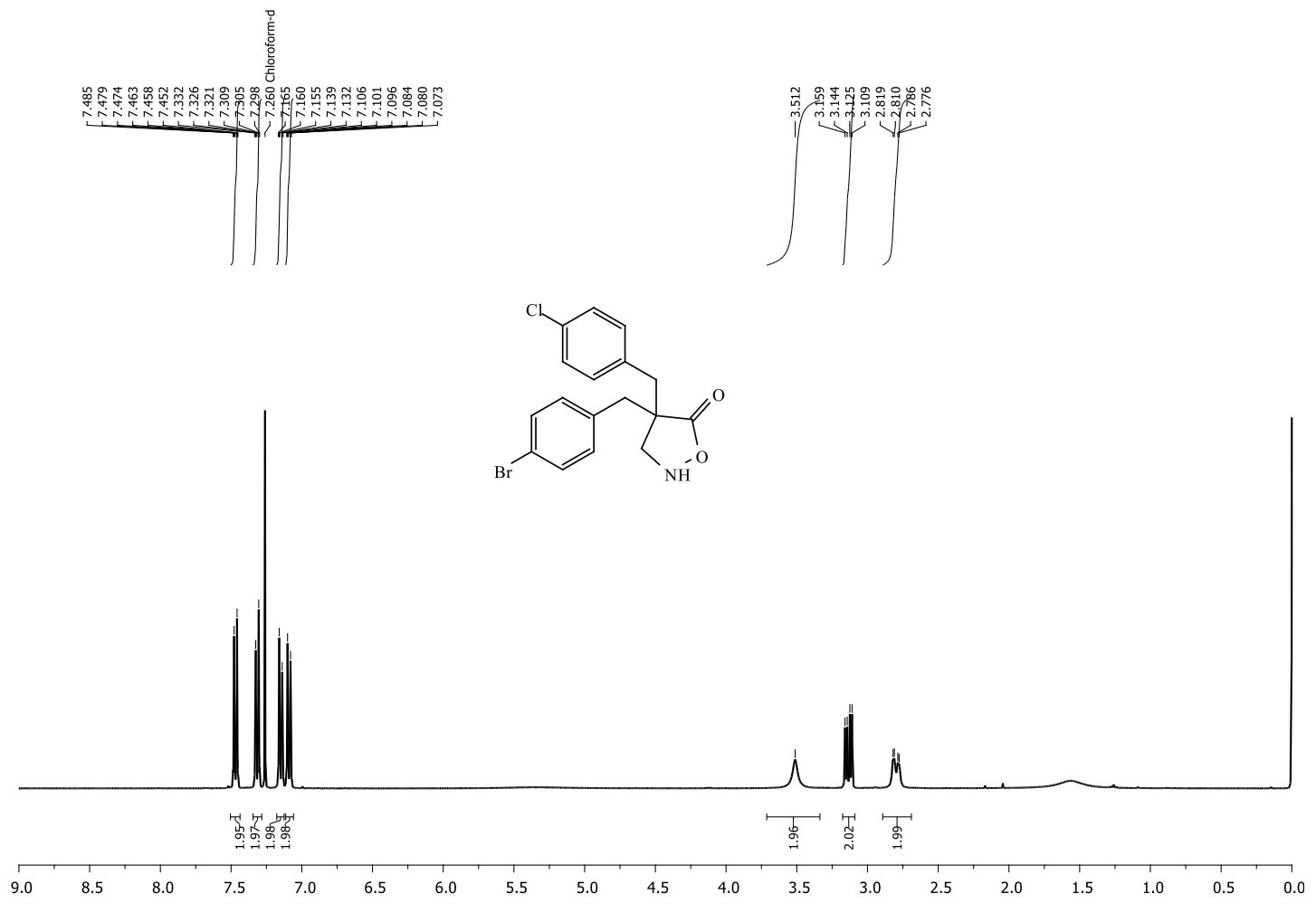

${ }^{13} \mathrm{C}$ NMR (101 MHz, $\left.\mathrm{CDCl}_{3}\right)$
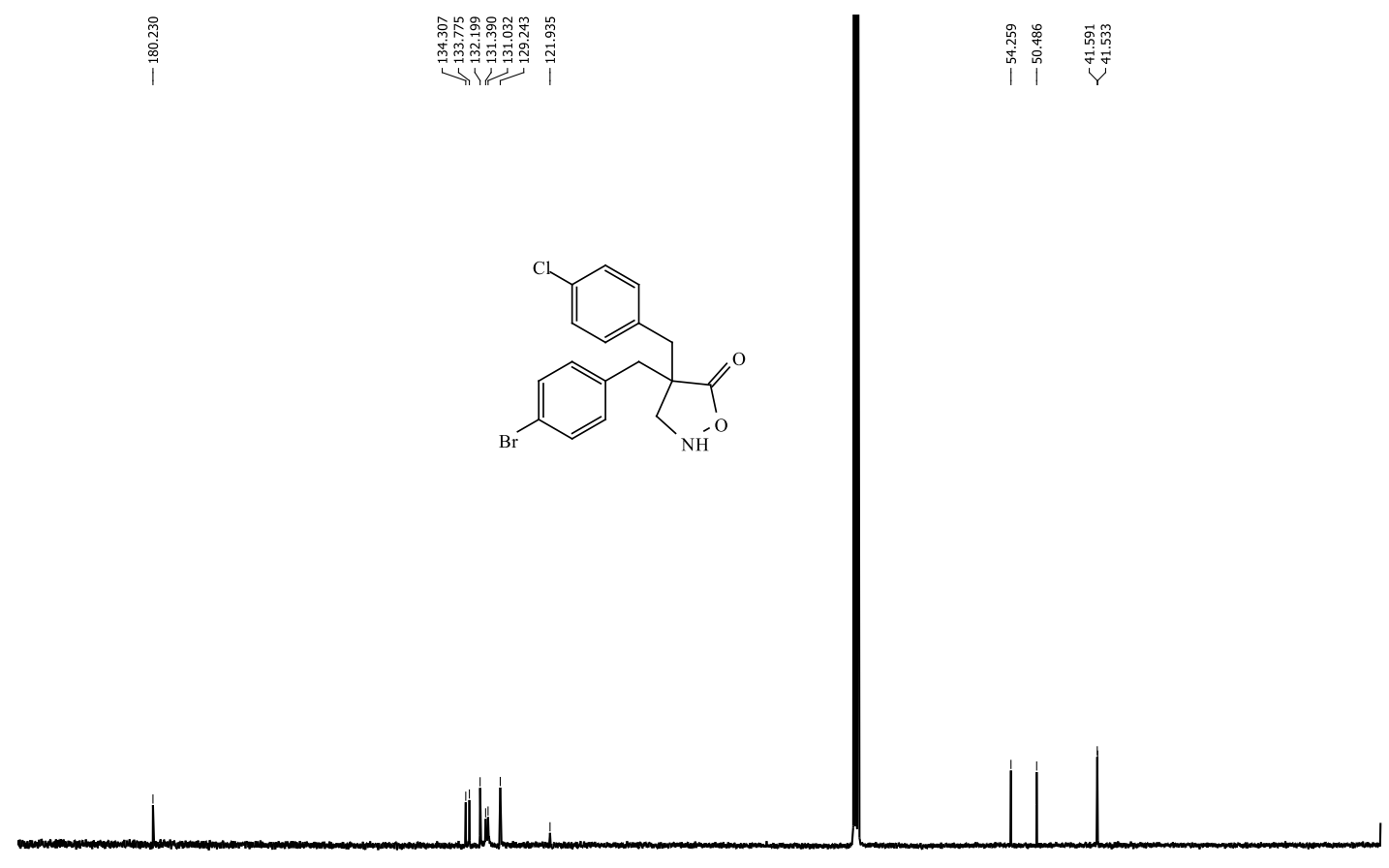
4-(Cyclohexylmethyl)-4-(4-methylbenzyl)isoxazolidin-5-one (7b)

${ }^{1}$ H NMR (400 $\mathrm{MHz}, \mathrm{CDCl}_{3}$ )

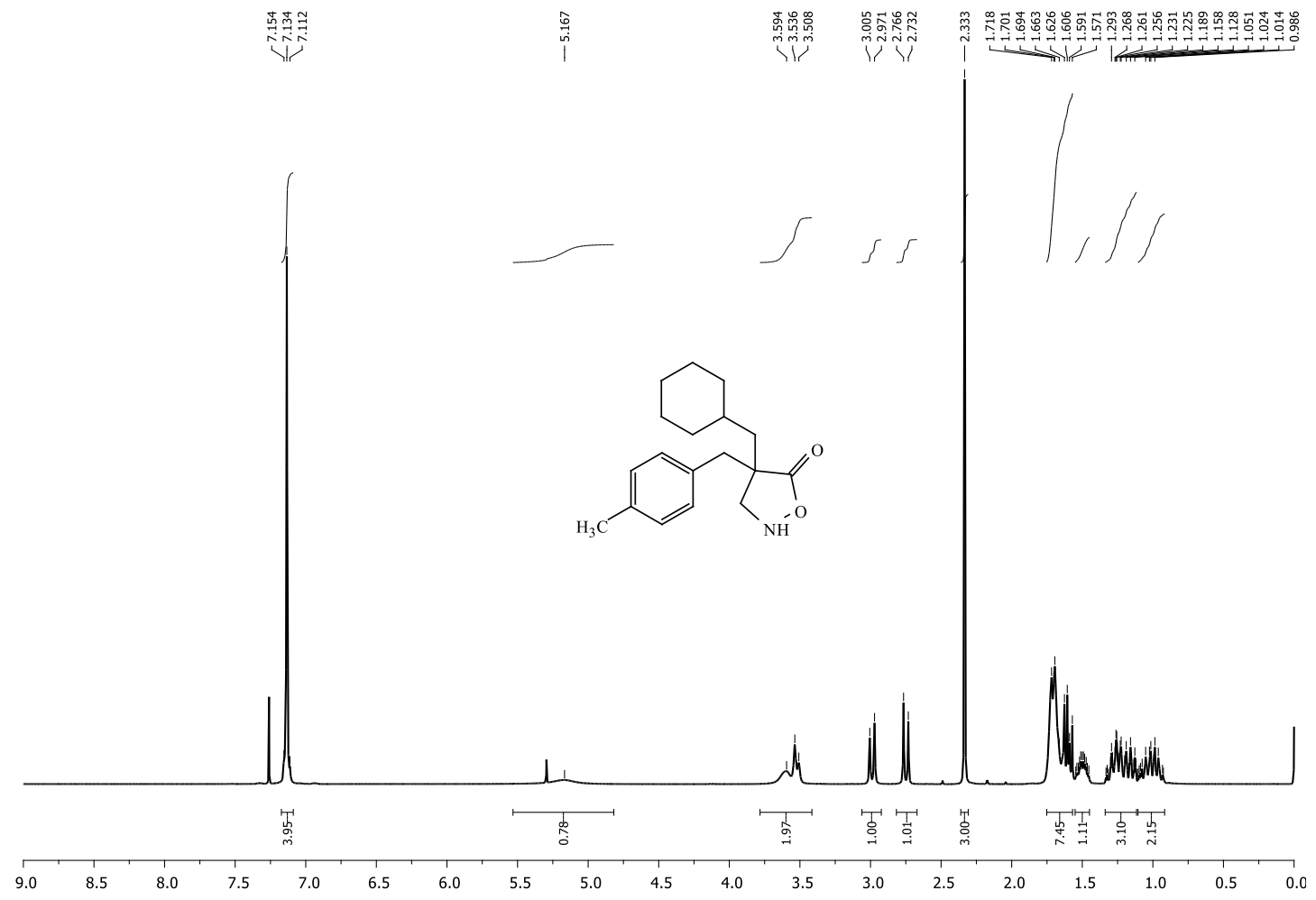

${ }^{13} \mathrm{C}$ NMR (101 MHz, $\left.\mathrm{CDCl}_{3}\right)$
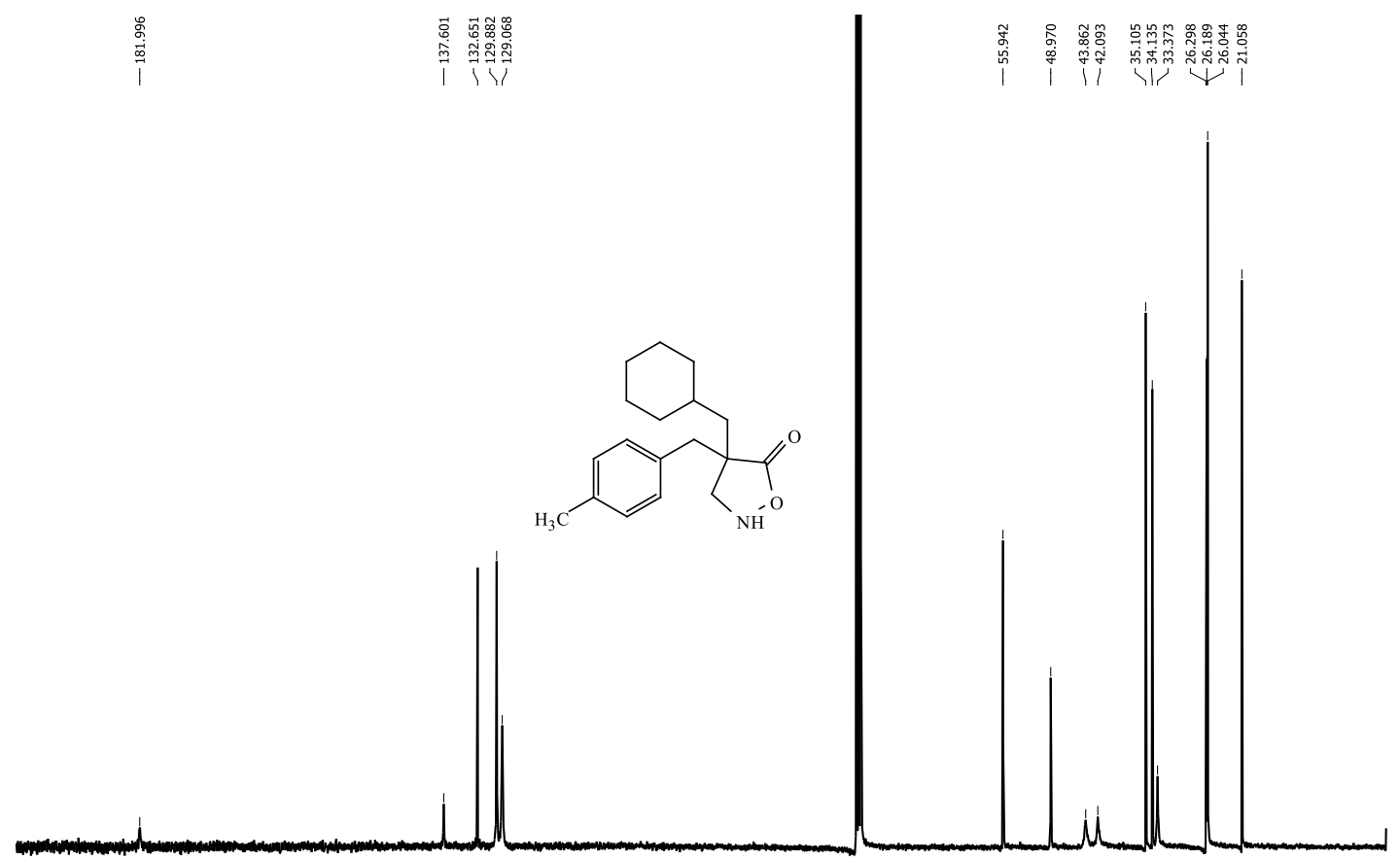

Page S74 of S104 


\section{4-(Cyclohexylmethyl)-4-(2-methylbenzyl)isoxazolidin-5-one (7c)}

${ }^{1} \mathrm{H}$ NMR (400 MHz, $\mathrm{CDCl}_{3}$ )
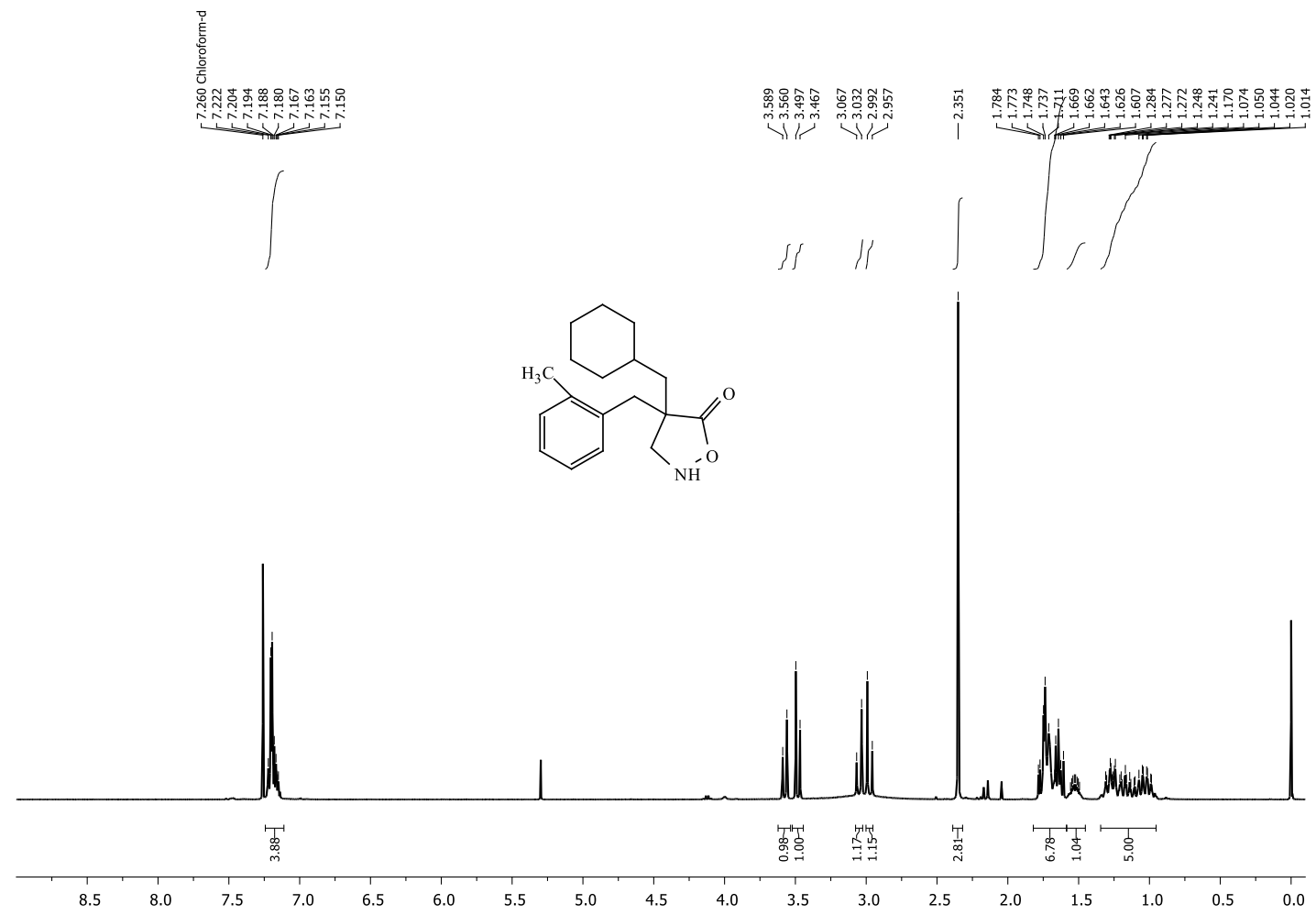

${ }^{13} \mathrm{C}$ NMR (101 MHz, $\left.\mathrm{CDCl}_{3}\right)$

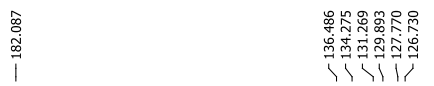

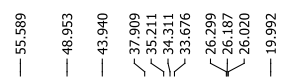
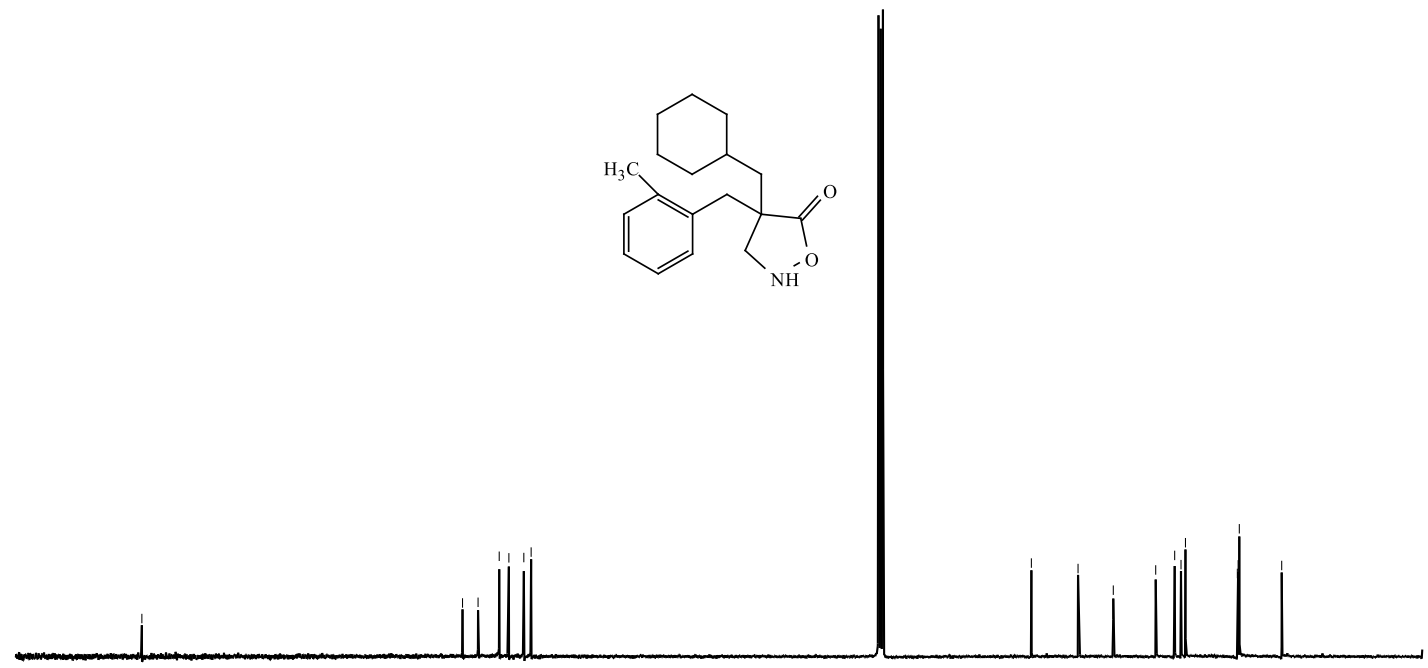
4-([1,1'-Biphenyl]-4-ylmethyl)-4-(cyclohexylmethyl)isoxazolidin-5-one (7d)

${ }^{1}$ H NMR (400 $\mathrm{MHz}, \mathrm{CDCl}_{3}$ )

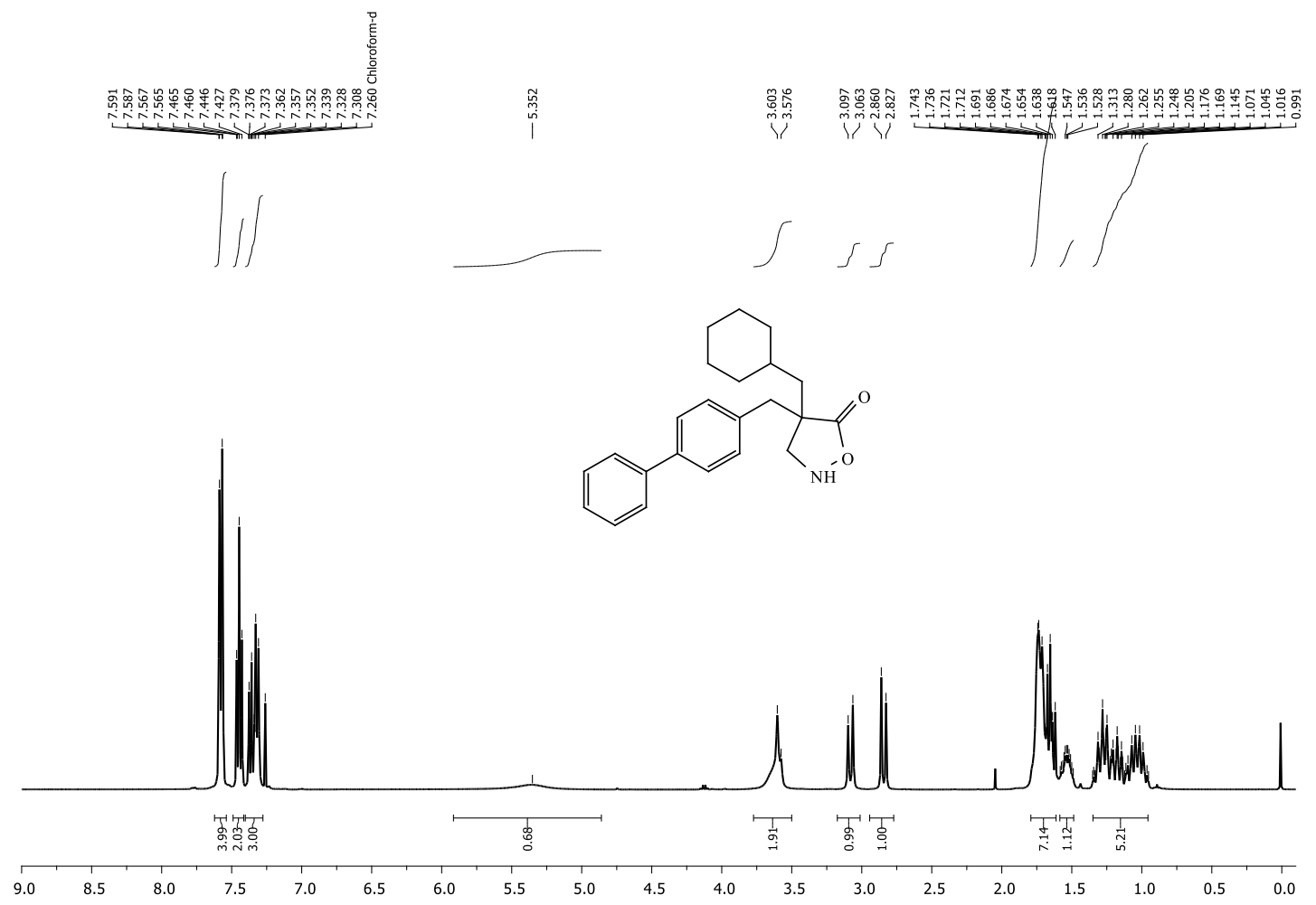

${ }^{13} \mathrm{C}$ NMR (101 MHz, $\left.\mathrm{CDCl}_{3}\right)$
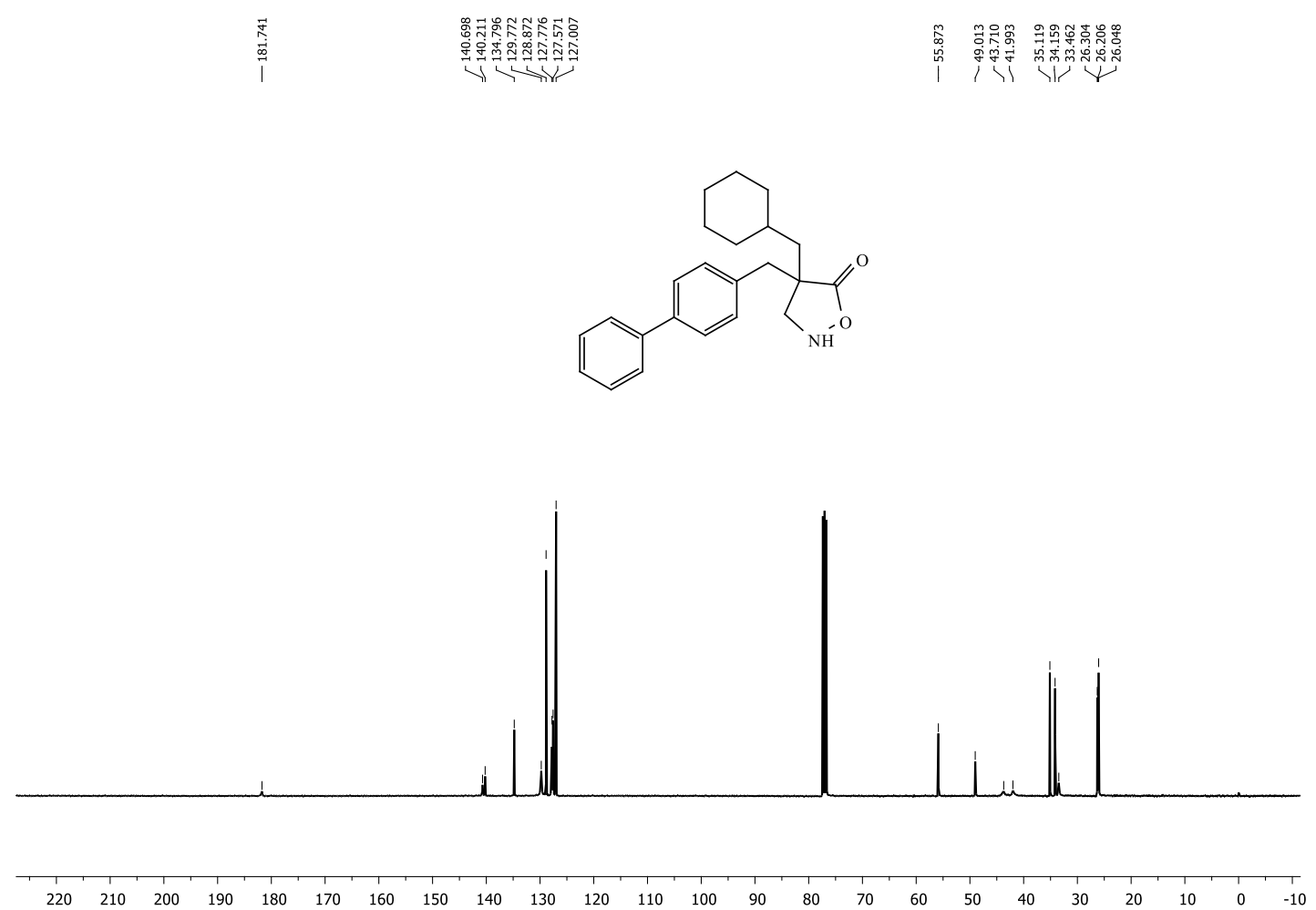
4-Benzyl-4-((tetrahydro-2H-pyran-4-yl)methyl)isoxazolidin-5-one (7e)

${ }^{1}$ H NMR (400 MHz, $\mathrm{CDCl}_{3}$ )
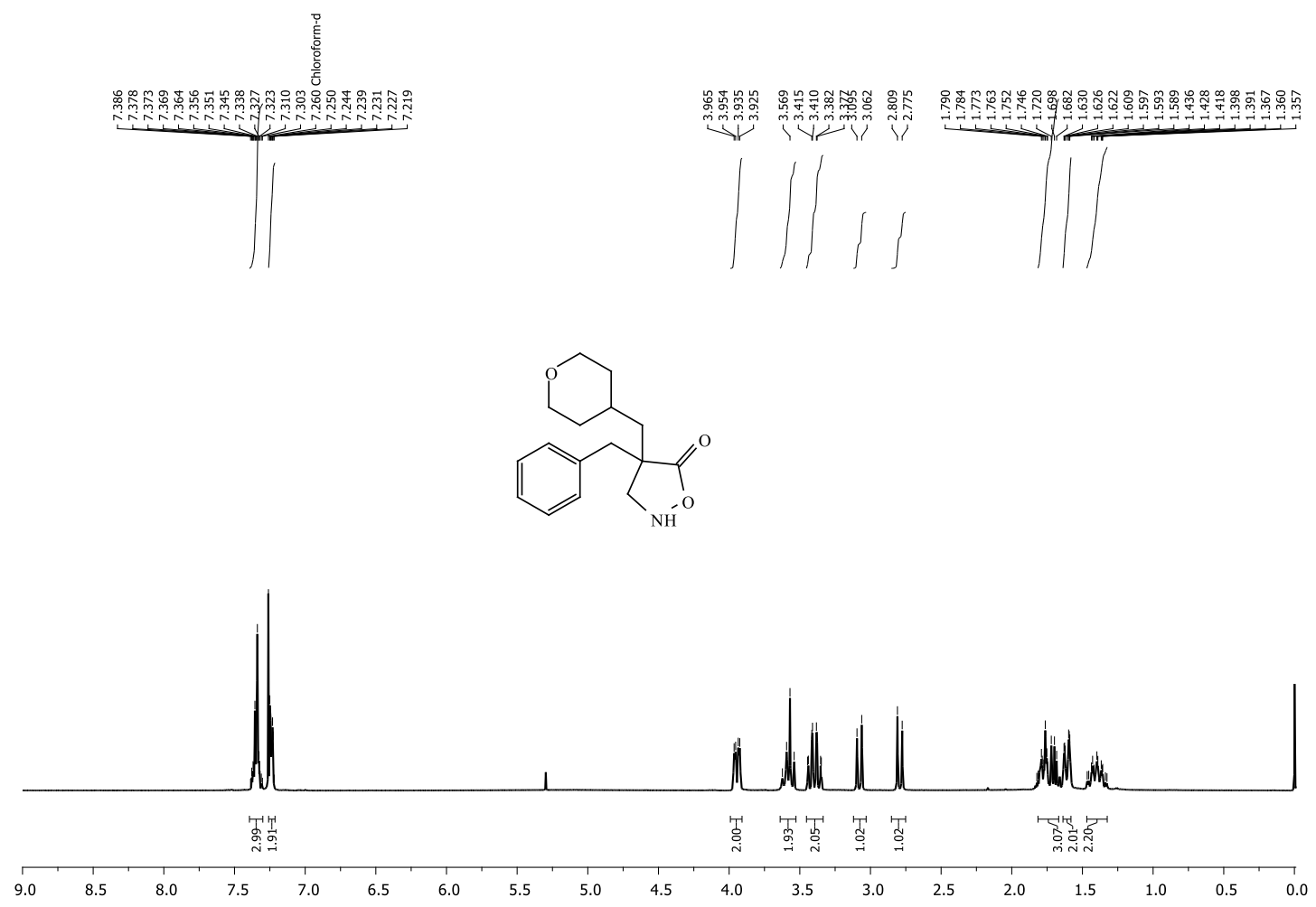

${ }^{13} \mathrm{C}$ NMR (101 MHz, $\left.\mathrm{CDCl}_{3}\right)$
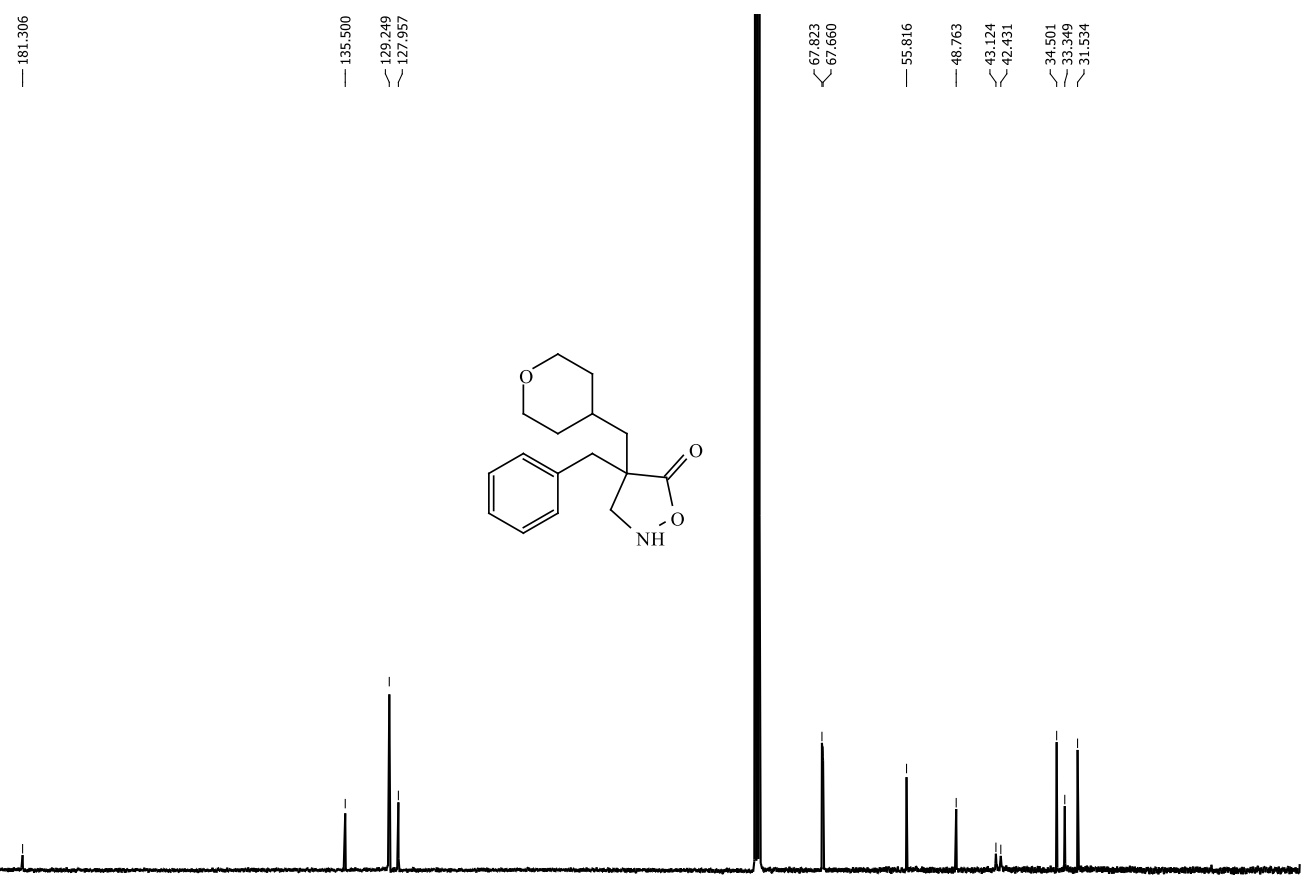

200

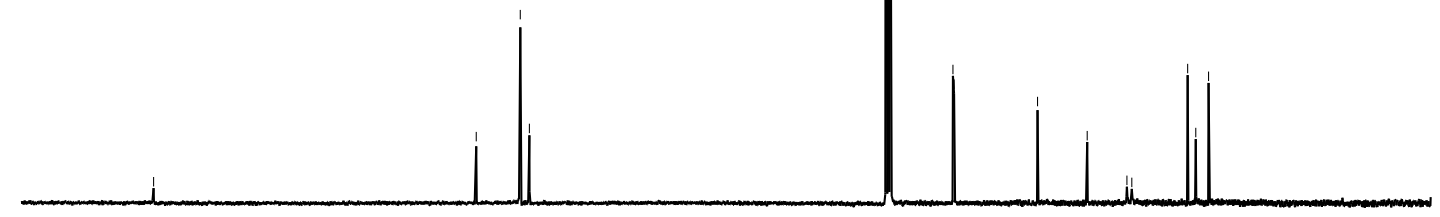

Page S77 of S104 
4-Benzyl-4-(4-nitrobenzyl)isoxazolidin-5-one (7f)

${ }^{1}$ H NMR (400 MHz, $\mathrm{CDCl}_{3}$ )

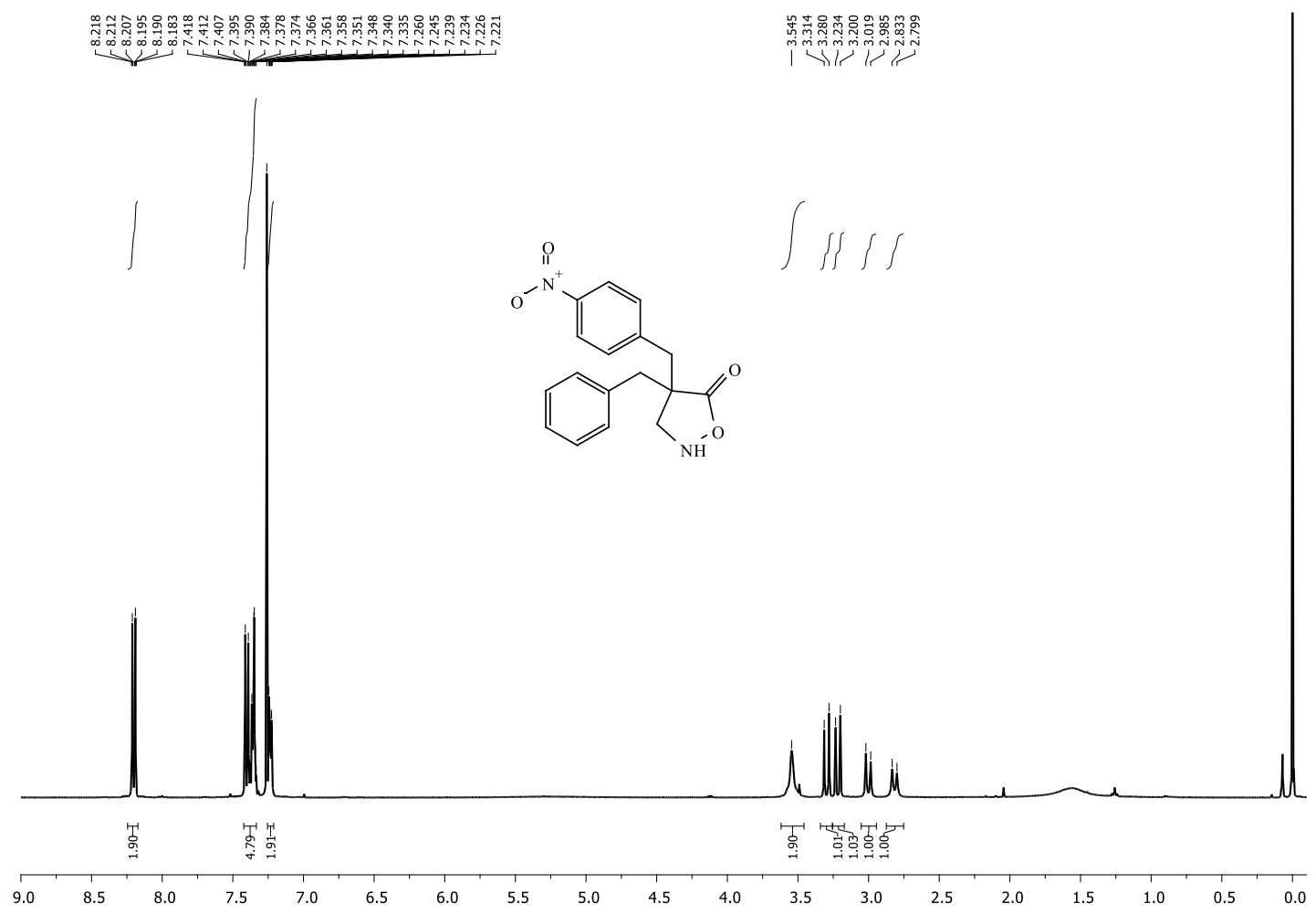

${ }^{13} \mathrm{C}$ NMR (101 MHz, $\left.\mathrm{CDCl}_{3}\right)$
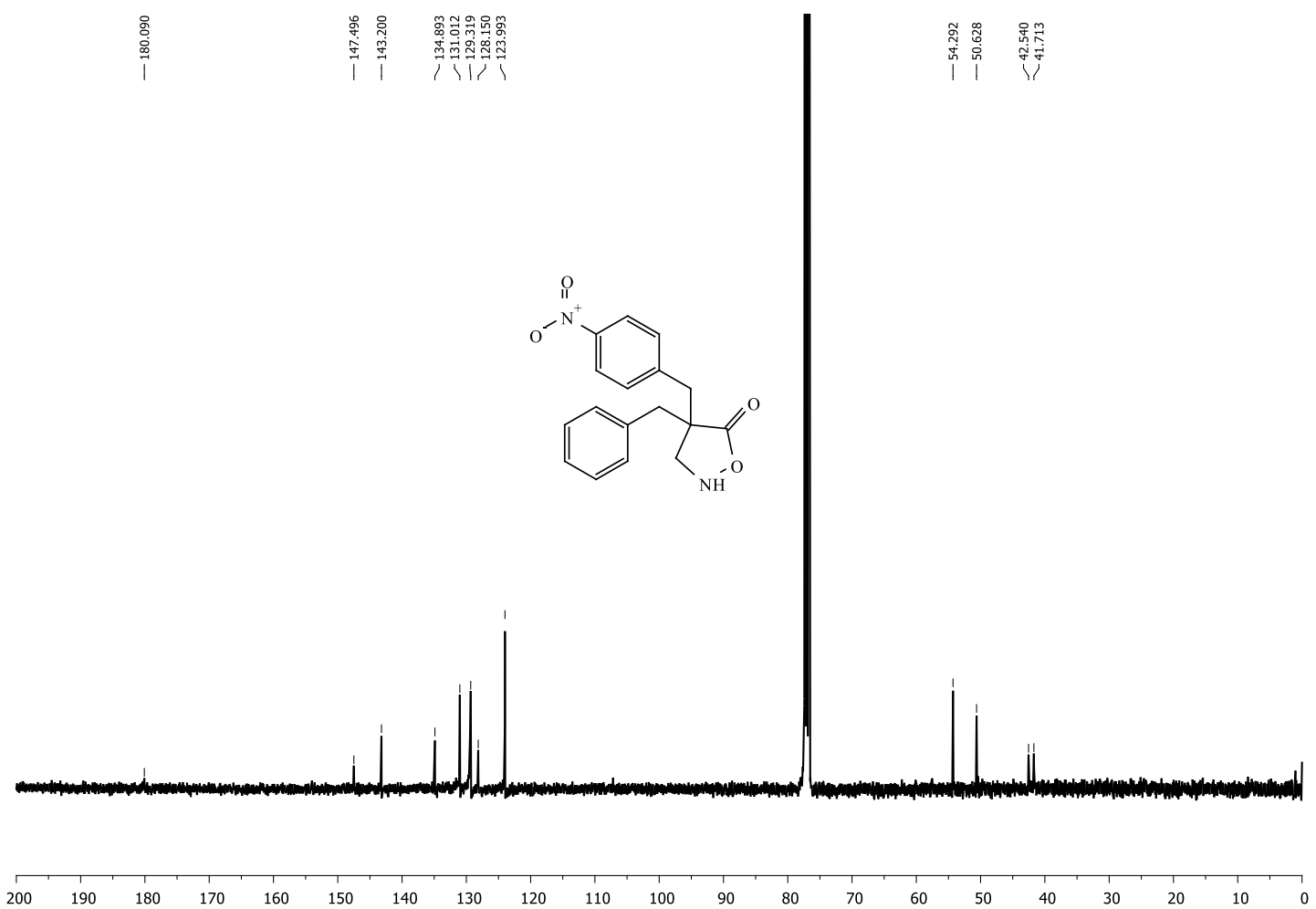

Page S78 of S104 
Methyl (R)-3-benzyl-1,2,3,4-tetrahydroquinoline-3-carboxylate (2a)

${ }^{1} \mathrm{H}$ NMR (400 MHz, $\mathrm{CDCl}_{3}$ )

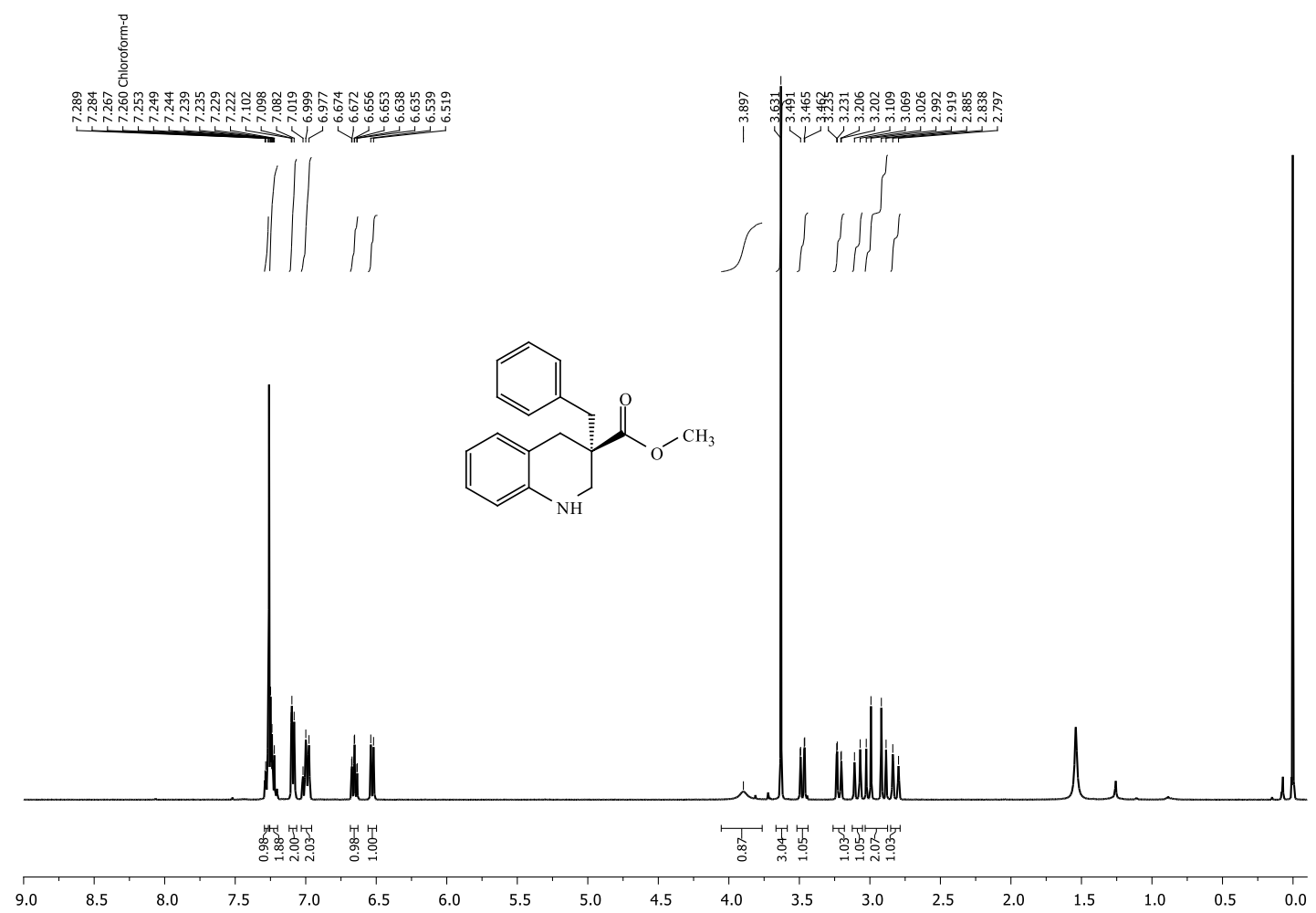

${ }^{13} \mathrm{C}$ NMR (101 MHz, $\left.\mathrm{CDCl}_{3}\right)$

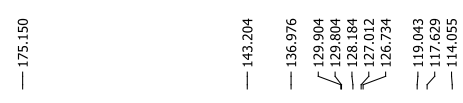

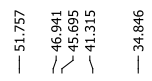
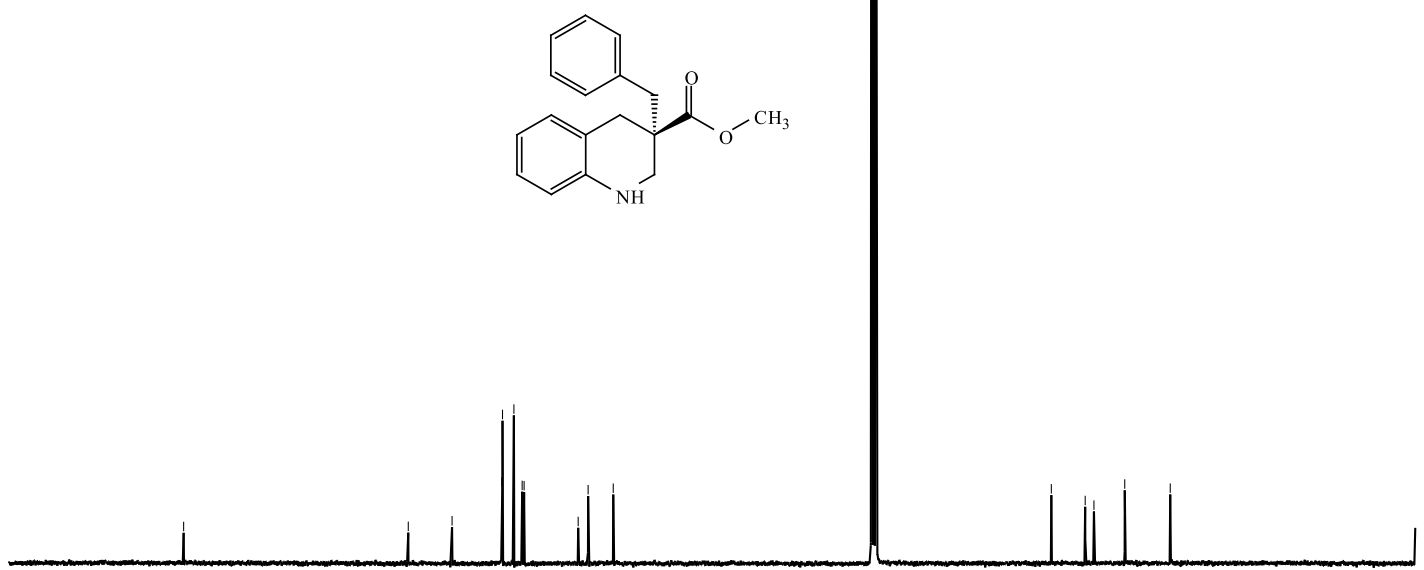


\section{Methyl (R)-7-methyl-3-(4-methylbenzyl)-1,2,3,4-tetrahydroquinoline-3-carboxylate (2b)}

${ }^{1} \mathrm{H}$ NMR (400 MHz, $\mathrm{CDCl}_{3}$ )

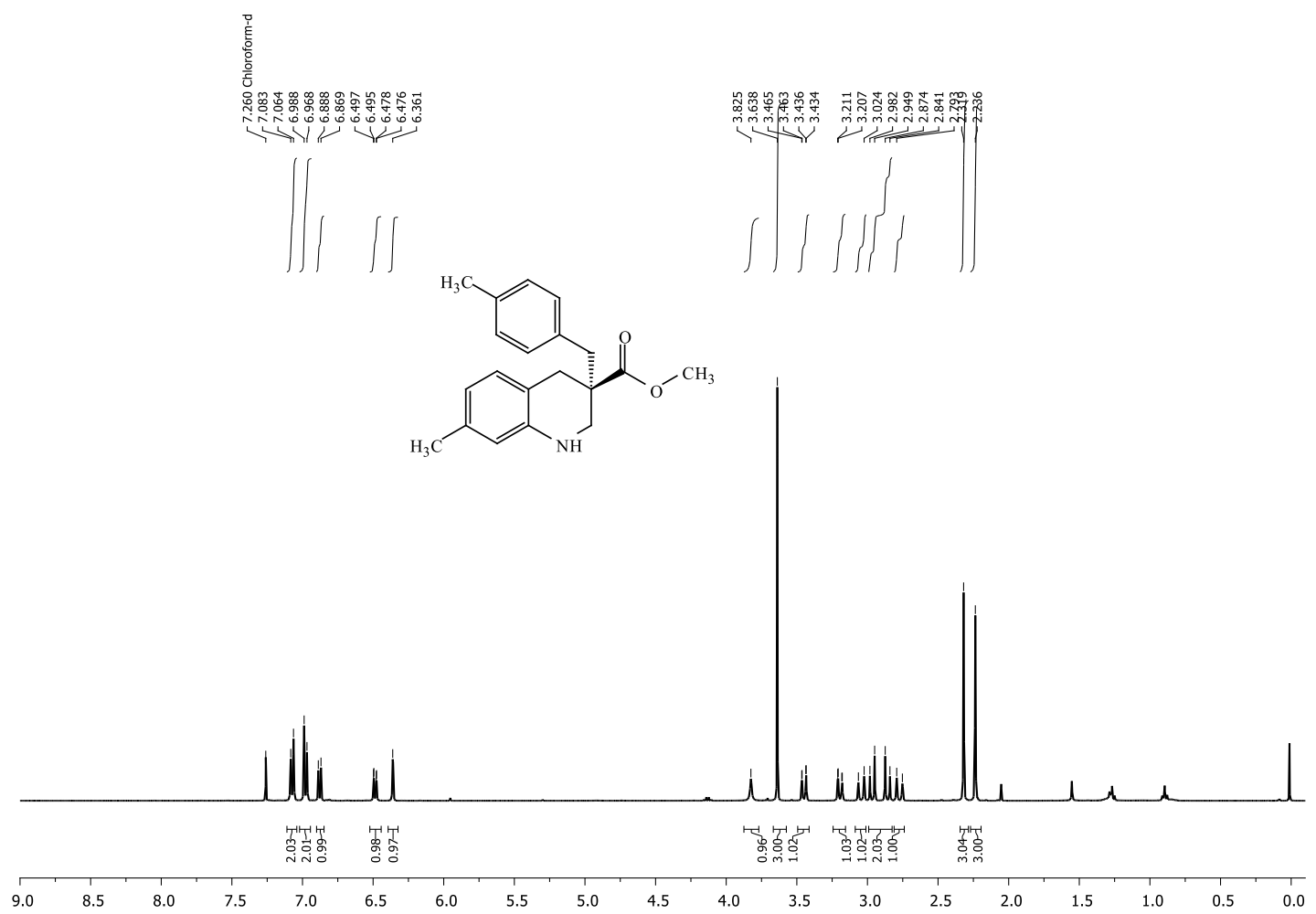

${ }^{13} \mathrm{C}$ NMR (101 MHz, $\left.\mathrm{CDCl}_{3}\right)$
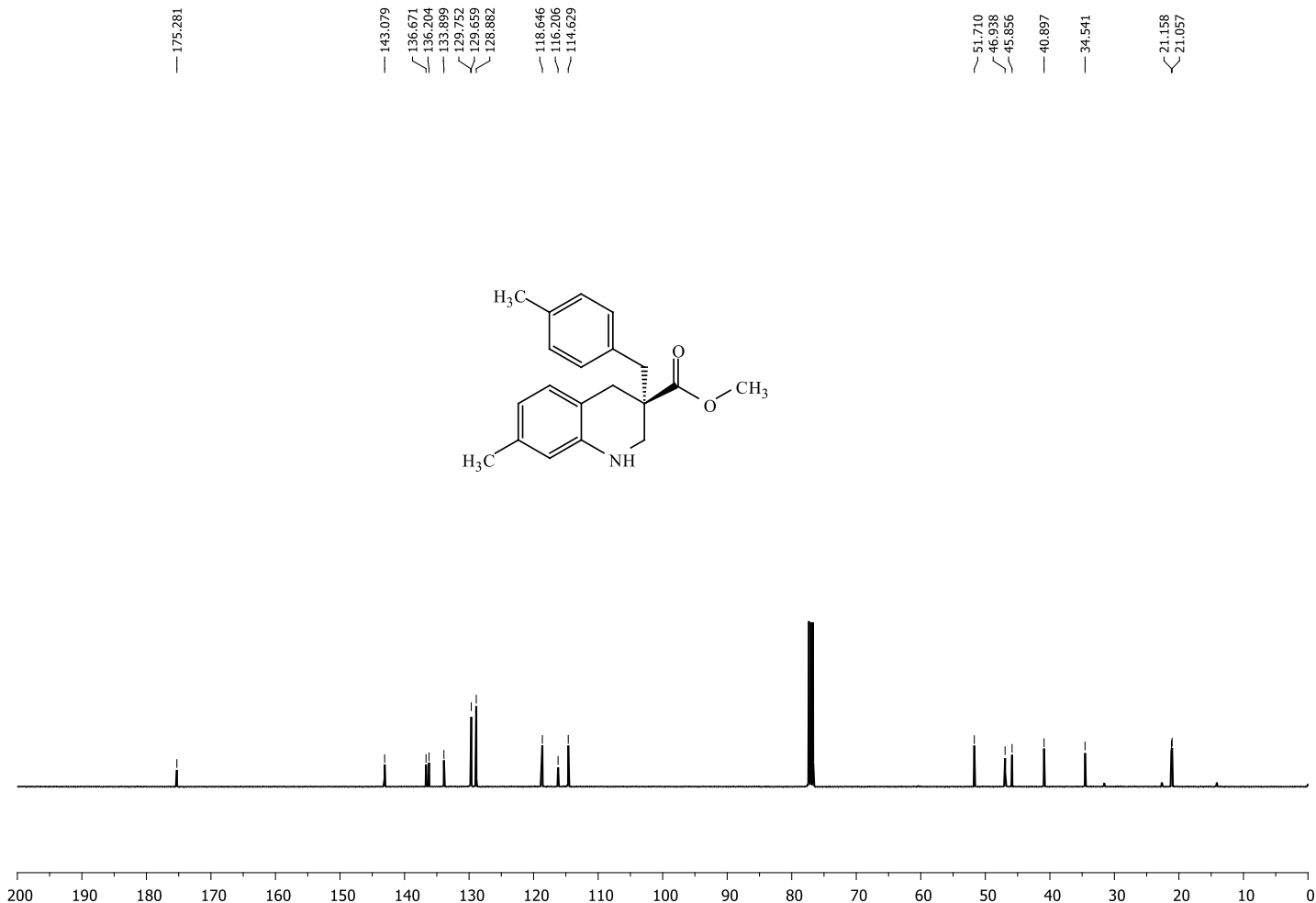

$\begin{array}{rrrrr}140 & 130 & 120 & 110 & 100\end{array}$

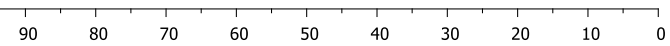

Page S80 of S104 
Methyl (R)-5-methyl-3-(2-methylbenzyl)-1,2,3,4-tetrahydroquinoline-3-carboxylate (2c)

${ }^{1} \mathrm{H}$ NMR (400 MHz, $\mathrm{CDCl}_{3}$ )
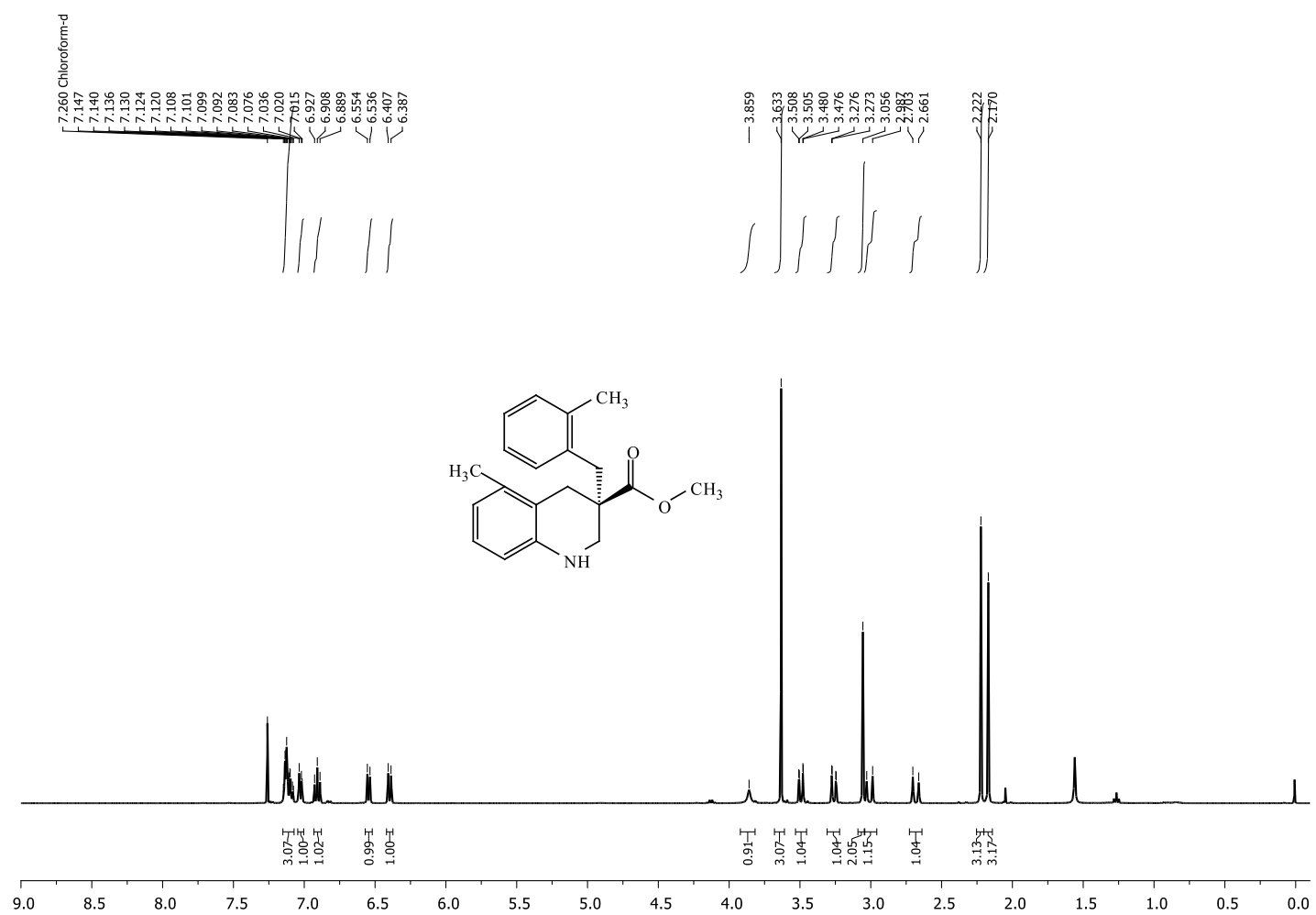

${ }^{13} \mathrm{C}$ NMR (101 MHz, $\left.\mathrm{CDCl}_{3}\right)$
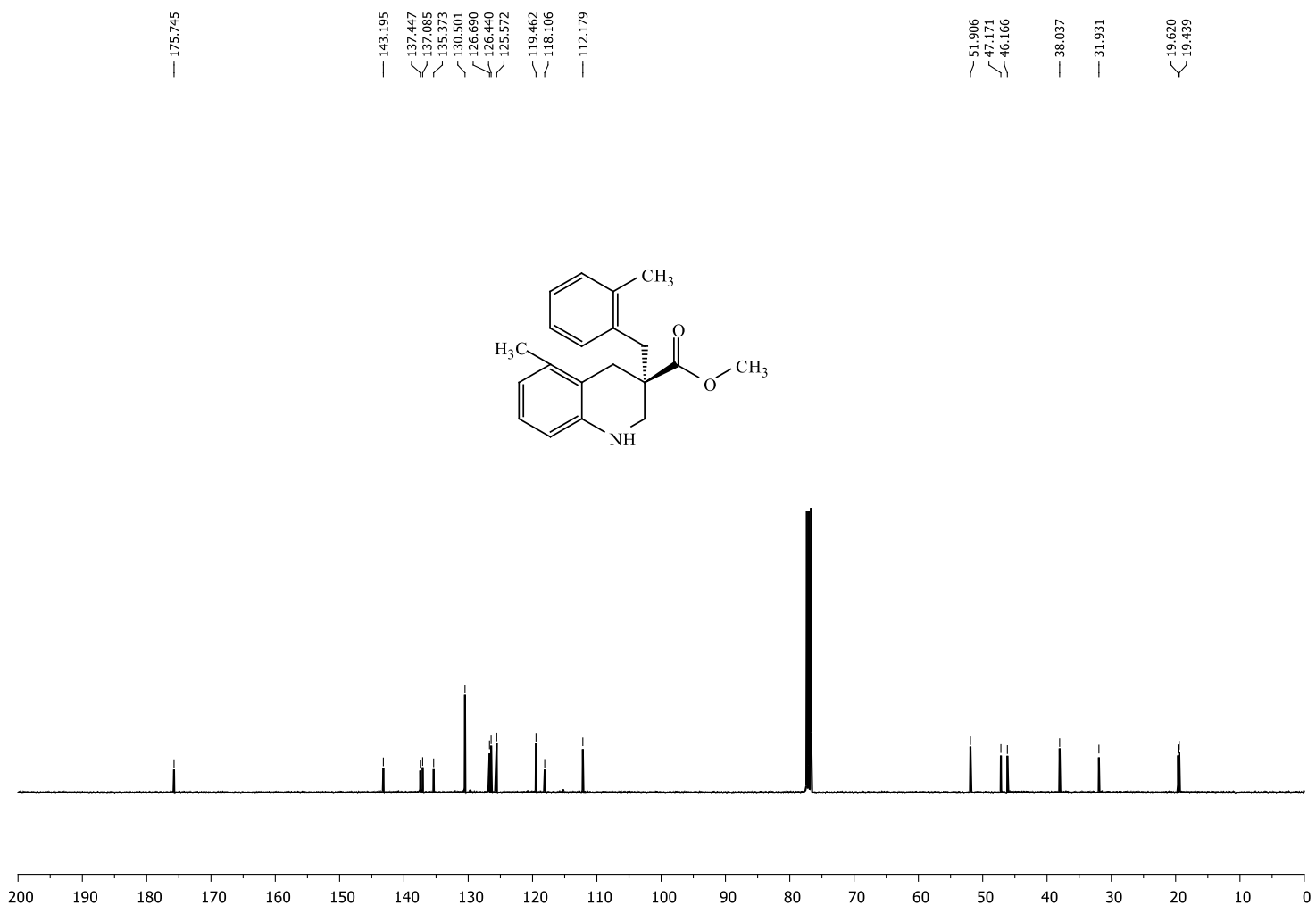

Page S81 of S104 
Methyl (R)-3-([1,1'-biphenyl]-4-ylmethyl)-7-phenyl-1,2,3,4-tetrahydroquinoline-3-carboxylate (2d)

${ }^{1} \mathrm{H}$ NMR (400 MHz, $\mathrm{CDCl}_{3}$ )
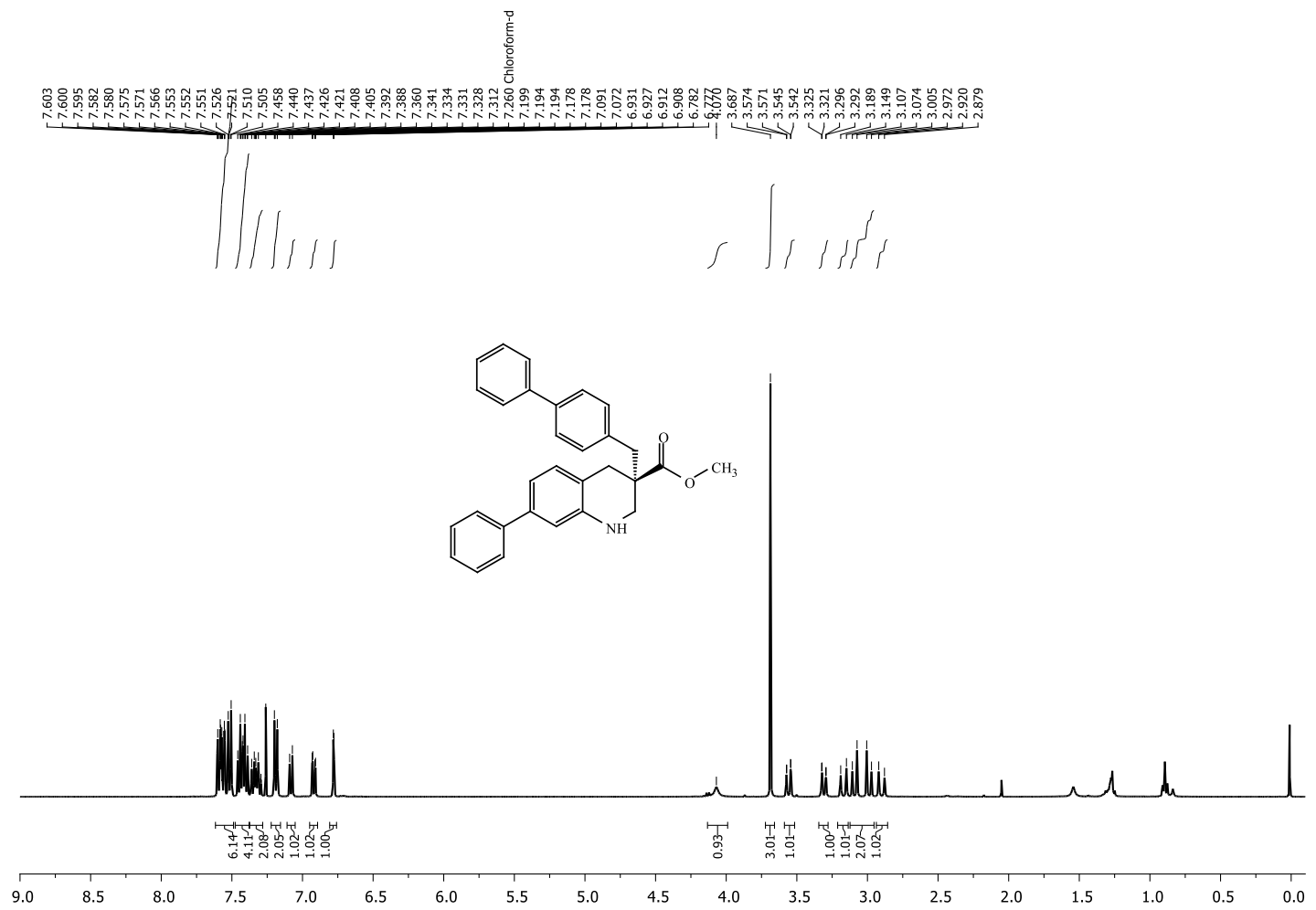

${ }^{13} \mathrm{C}$ NMR (101 MHz, $\left.\mathrm{CDCl}_{3}\right)$

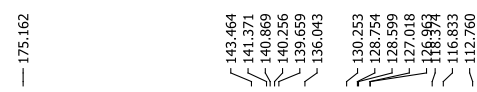

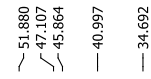
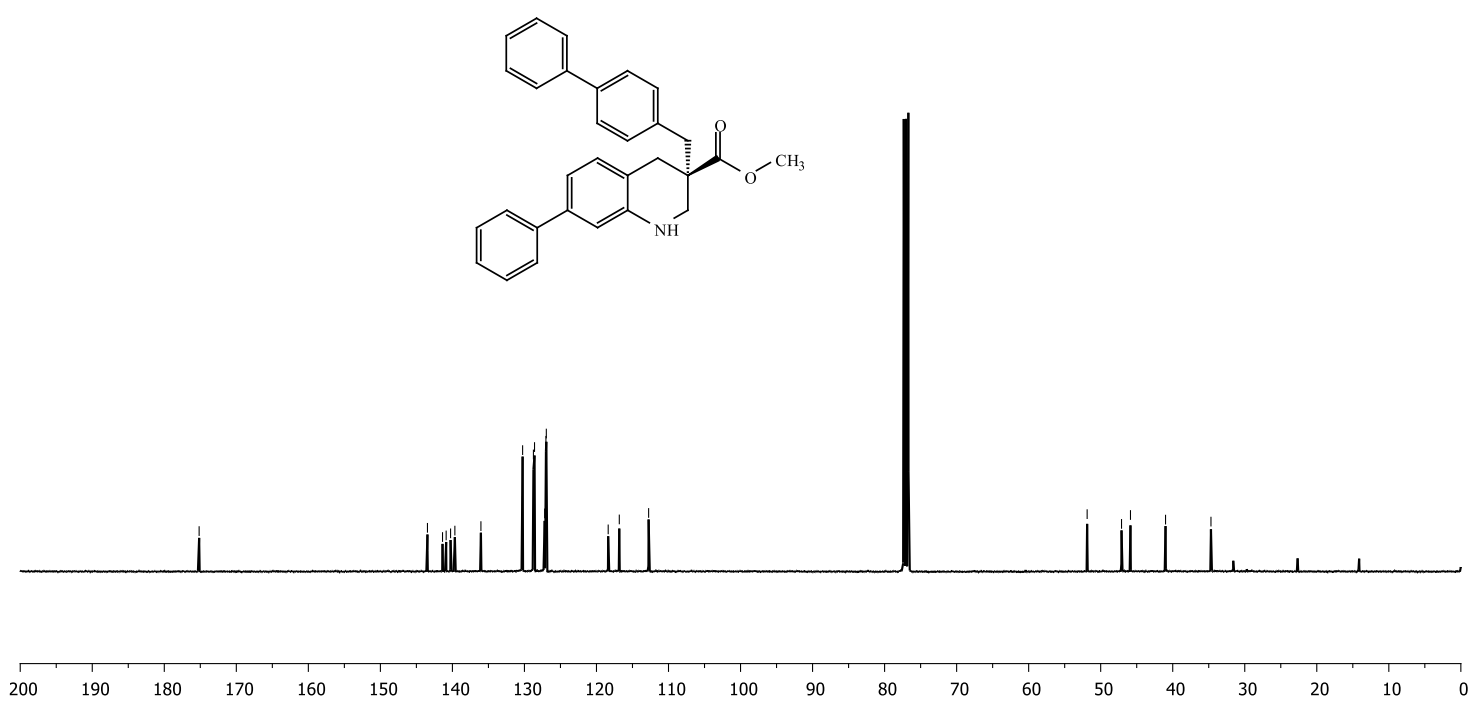

Page S82 of S104 
Methyl (R)-7-(tert-butyl)-3-(4-(tert-butyl)benzyl)-1,2,3,4-tetrahydroquinoline-3-carboxylate (2e) ${ }^{1} \mathrm{H}$ NMR (400 MHz, $\mathrm{CDCl}_{3}$ )

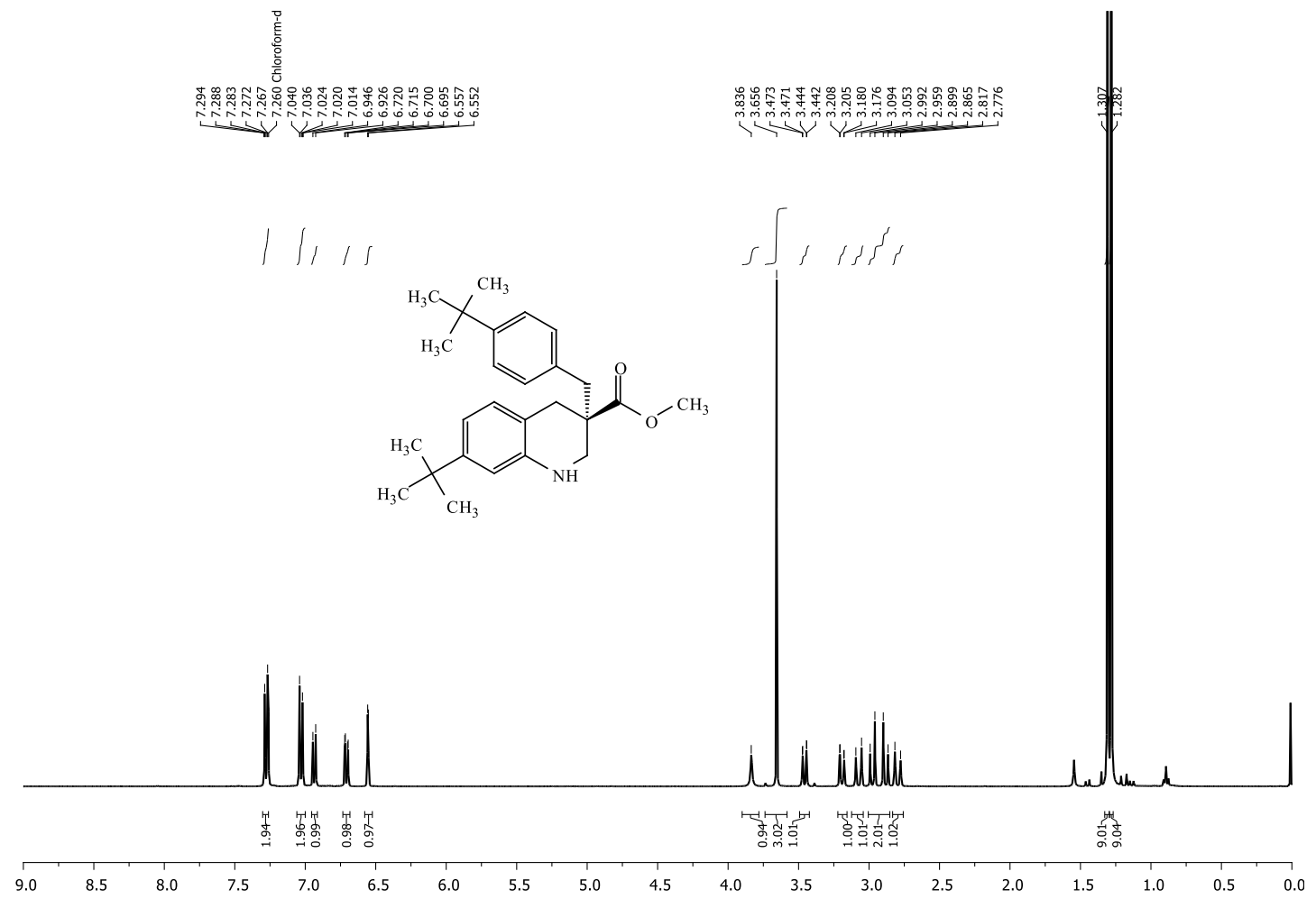

${ }^{13} \mathrm{C}$ NMR (101 MHz, $\left.\mathrm{CDCl}_{3}\right)$

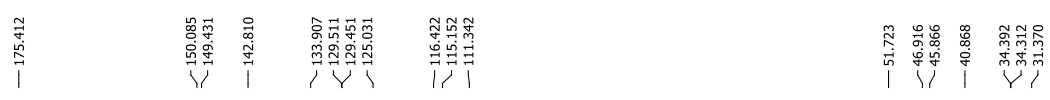
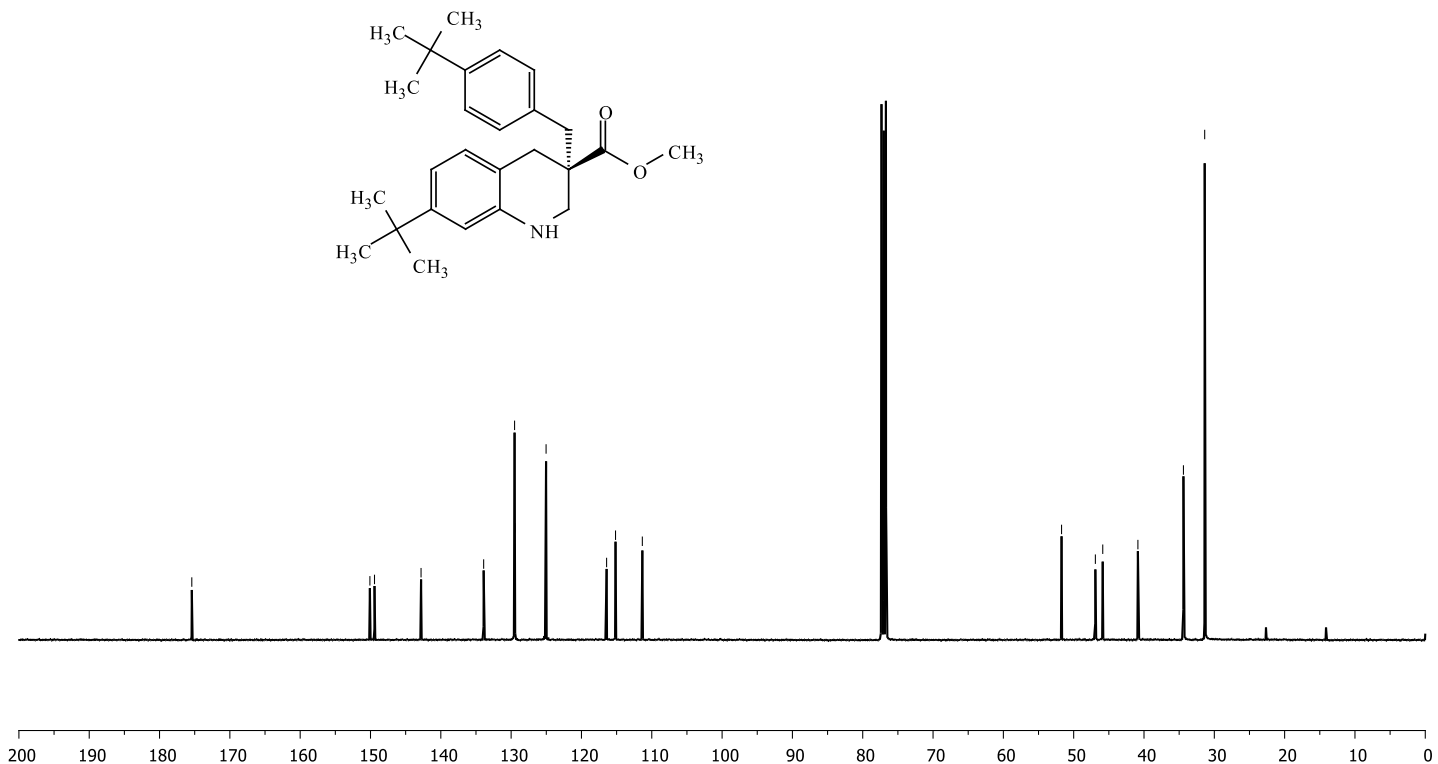

Page S83 of S104 
Methyl (R)-7-bromo-3-(4-bromobenzyl)-1,2,3,4-tetrahydroquinoline-3-carboxylate (2f)

${ }^{1} \mathrm{H}$ NMR (400 MHz, $\mathrm{CDCl}_{3}$ )
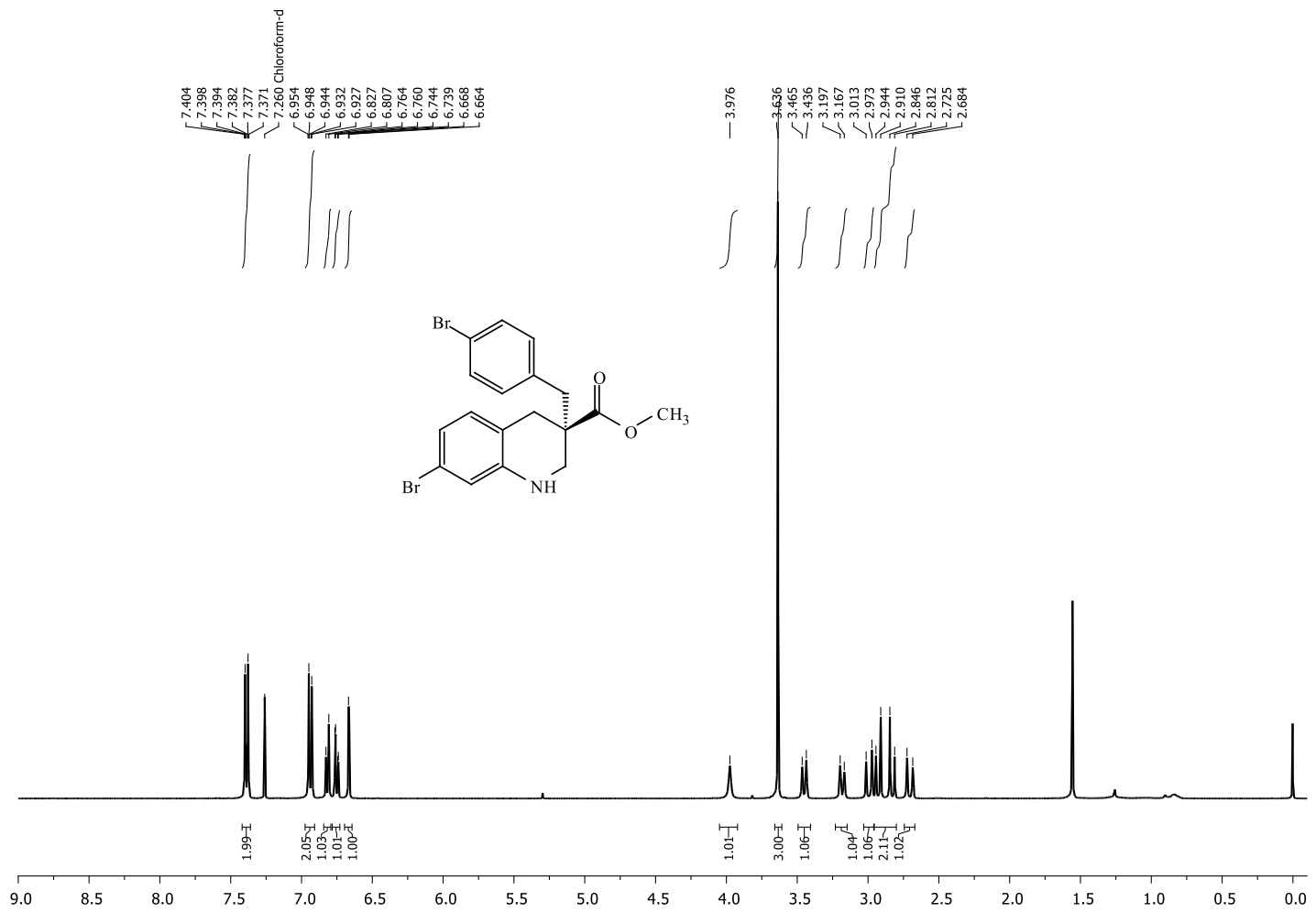

${ }^{13} \mathrm{C}$ NMR (101 MHz, $\left.\mathrm{CDCl}_{3}\right)$

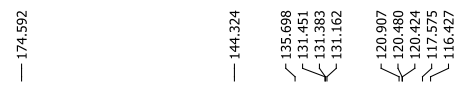

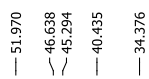
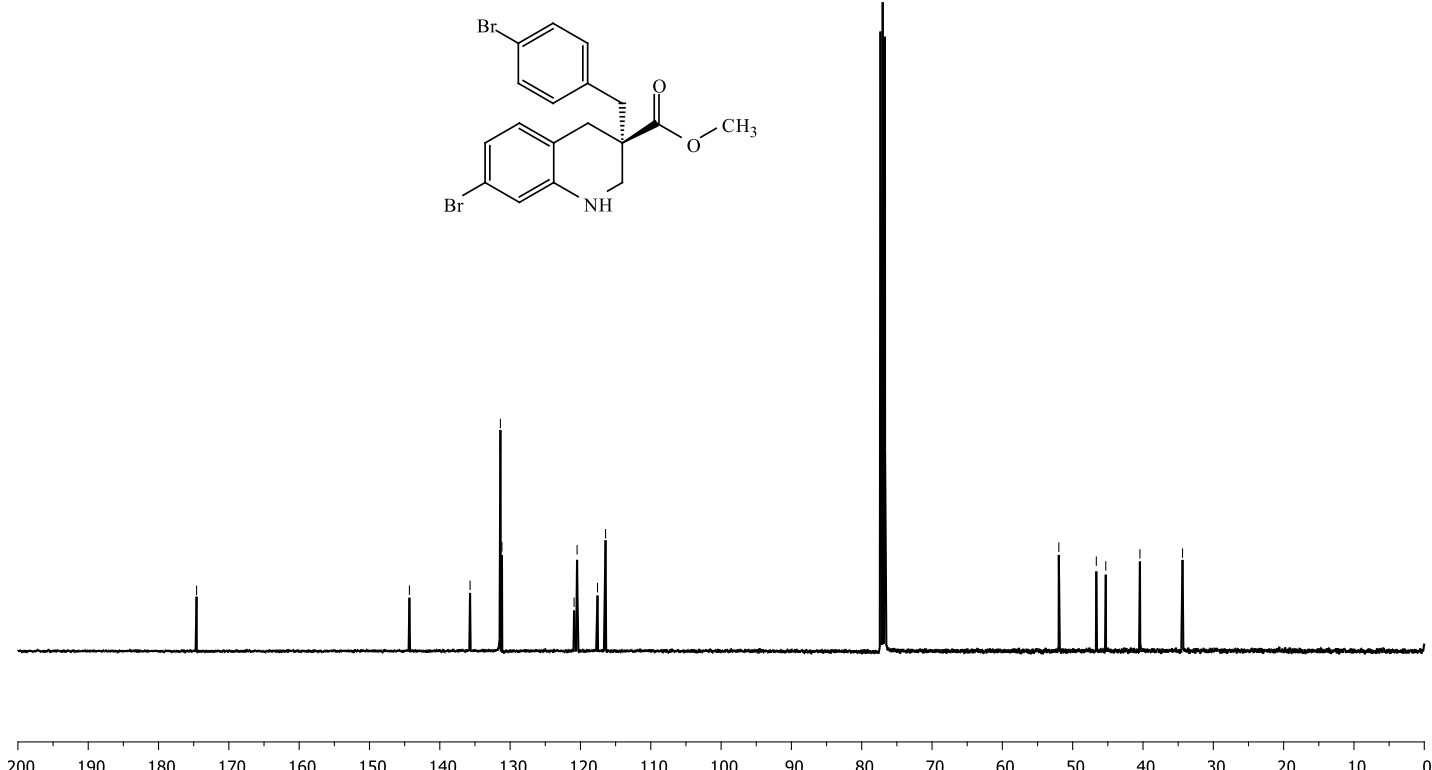
Methyl (R)-5-chloro-3-(2-chlorobenzyl)-1,2,3,4-tetrahydroquinoline-3-carboxylate (2g) ${ }^{1} \mathrm{H}$ NMR (400 MHz, $\mathrm{CDCl}_{3}$ )

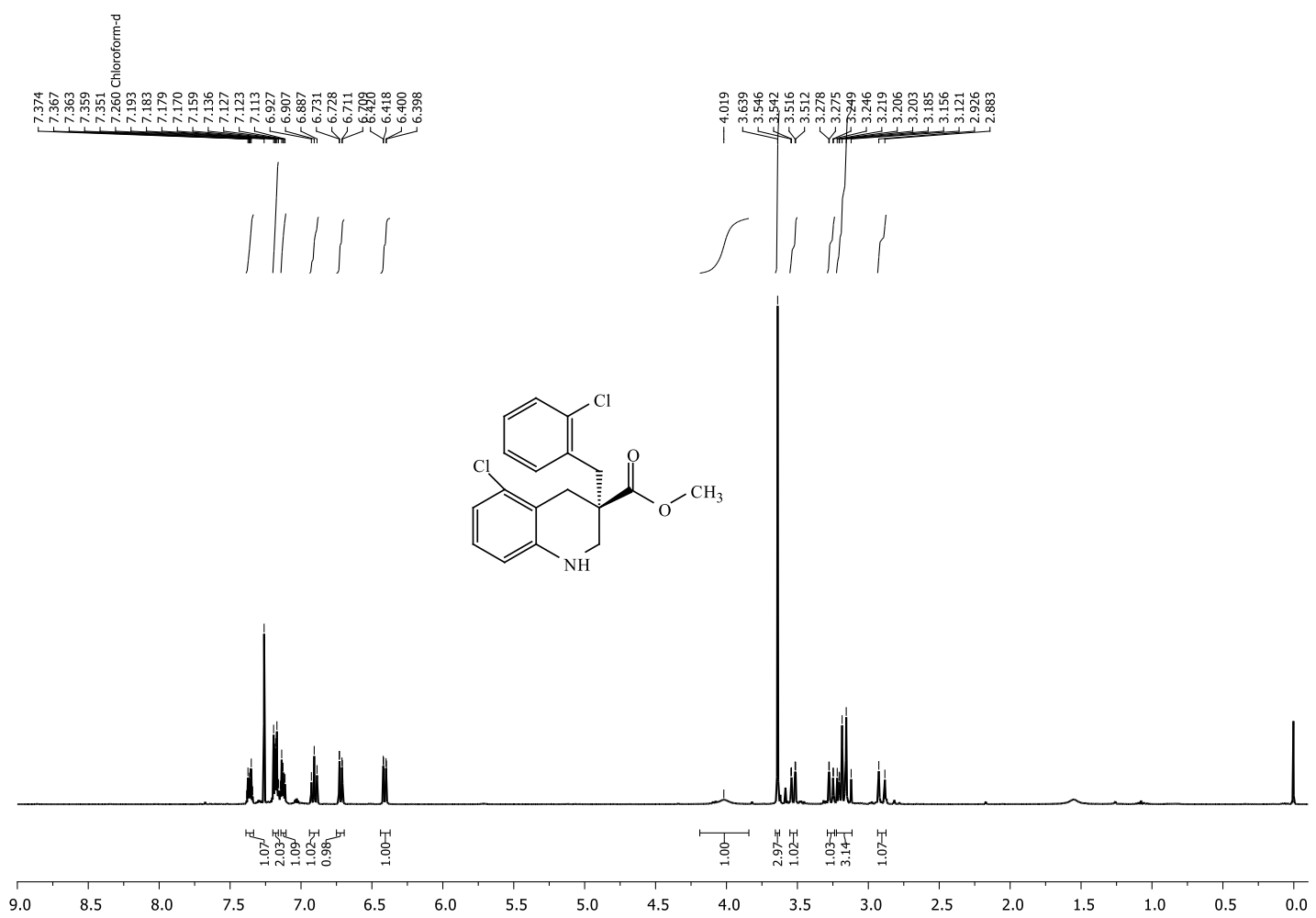

${ }^{13} \mathrm{C}$ NMR (101 MHz, $\left.\mathrm{CDCl}_{3}\right)$

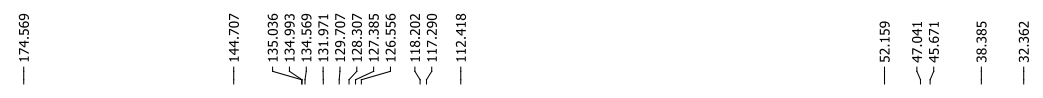

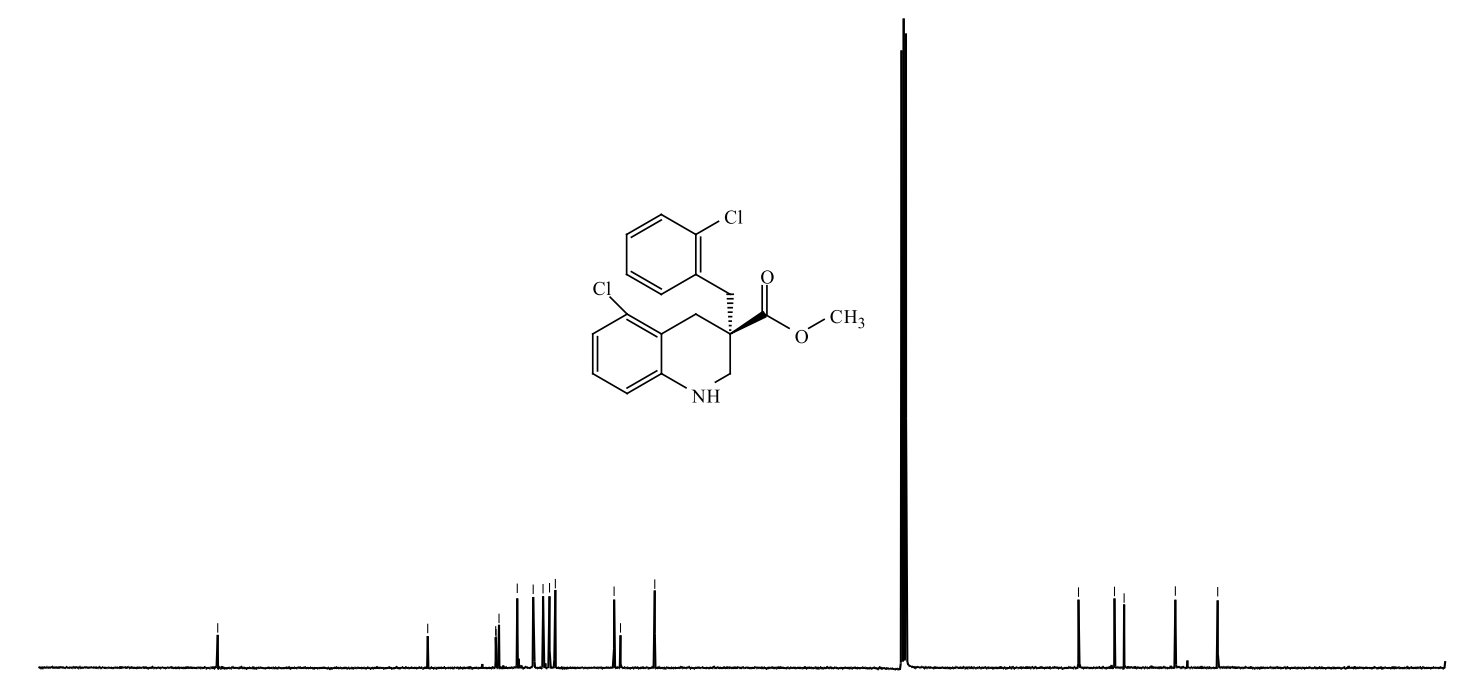

Page S85 of S104 
Mixture of methyl (R)-3-(4-methylbenzyl)-1,2,3,4-tetrahydroquinoline-3-carboxylate (6a-A) and methyl (R)3-benzyl-7-methyl-1,2,3,4-tetrahydroquinoline-3-carboxylate (6a-B)

${ }^{1} \mathrm{H}$ NMR (400 $\left.\mathrm{MHz}, \mathrm{CDCl}_{3}\right)$

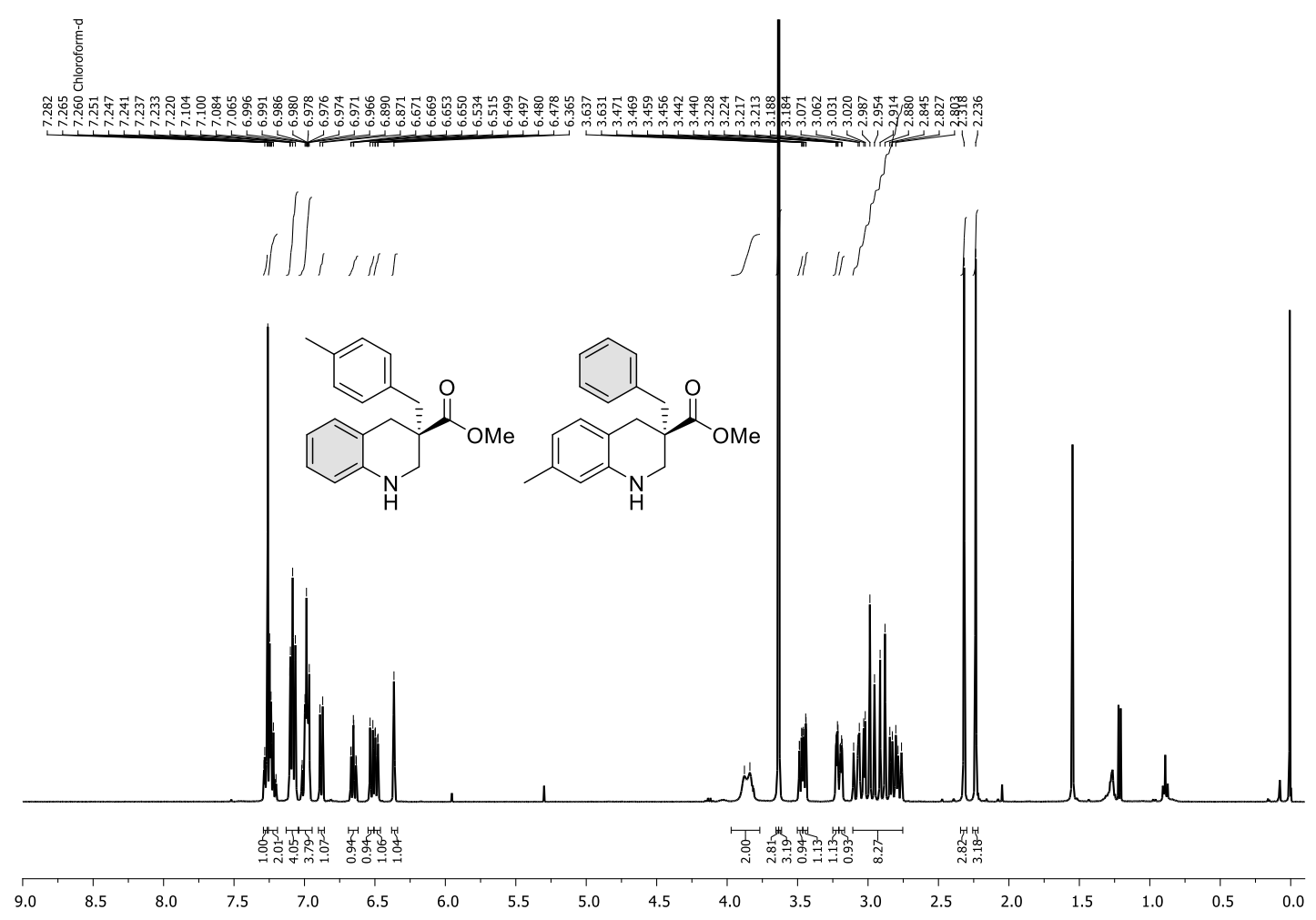

${ }^{13} \mathrm{C}$ NMR (101 MHz, $\left.\mathrm{CDCl}_{3}\right)$

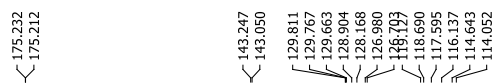

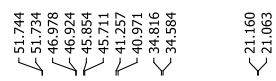<smiles>COC(=O)[C@@]1(Cc2ccccc2)CNc2cc(C)ccc2C1</smiles>

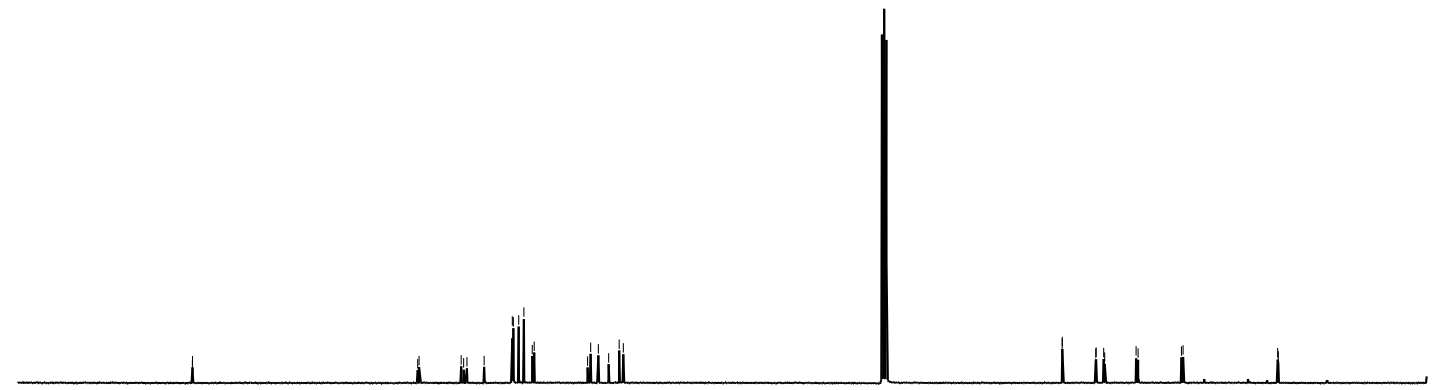

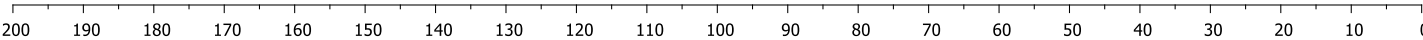


Mixture of methyl $(R)-3-([1,1$ '-biphenyl]-4-ylmethyl)-1,2,3,4-tetrahydroquinoline-3-carboxylate (6b-A) and methyl $(R)$-3-benzyl-7-phenyl-1,2,3,4-tetrahydroquinoline-3-carboxylate (6b-B)

${ }^{1} \mathrm{H}$ NMR (400 MHz, $\mathrm{CDCl}_{3}$ )

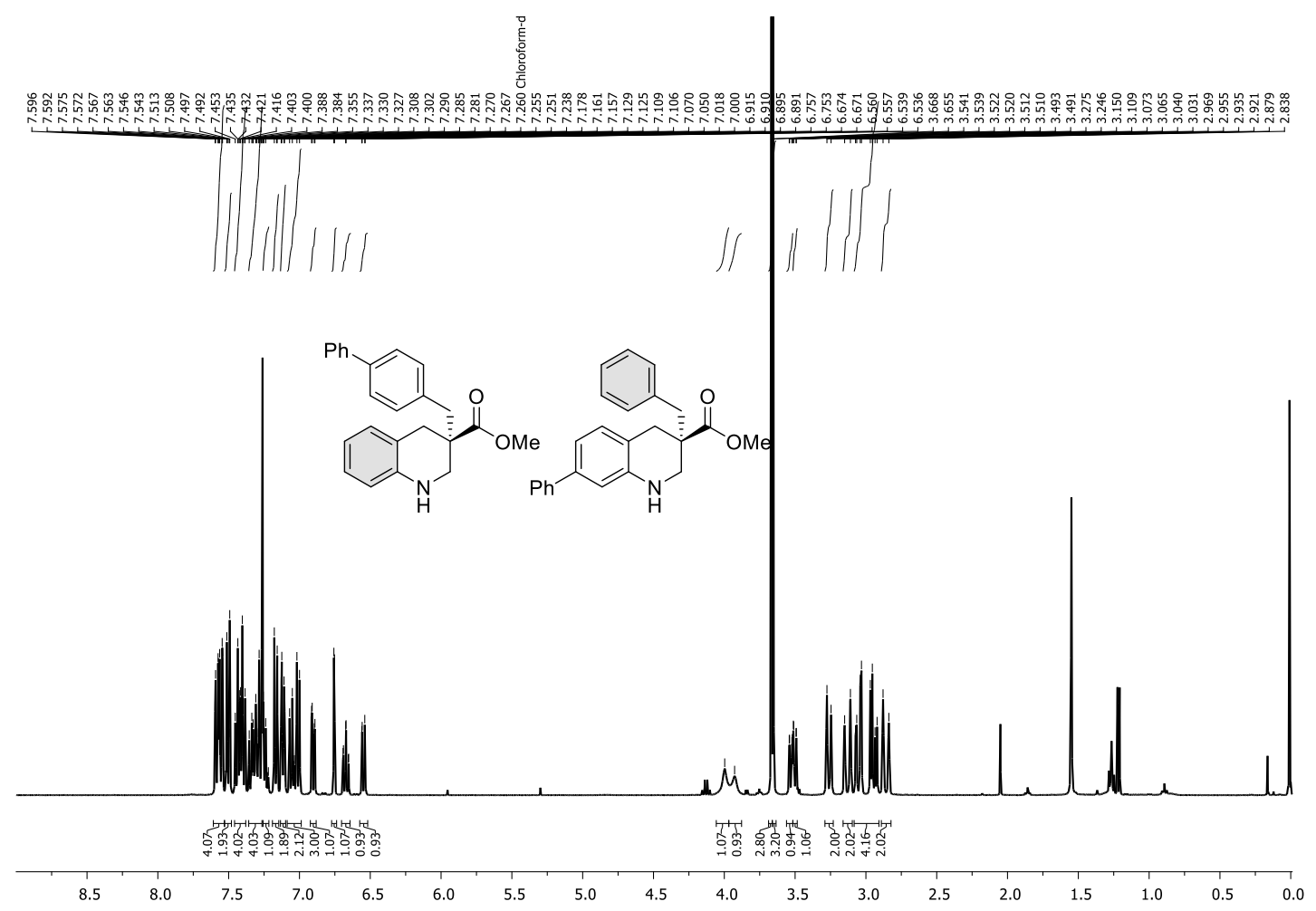

${ }^{13} \mathrm{C}$ NMR (101 MHz, $\left.\mathrm{CDCl}_{3}\right)$
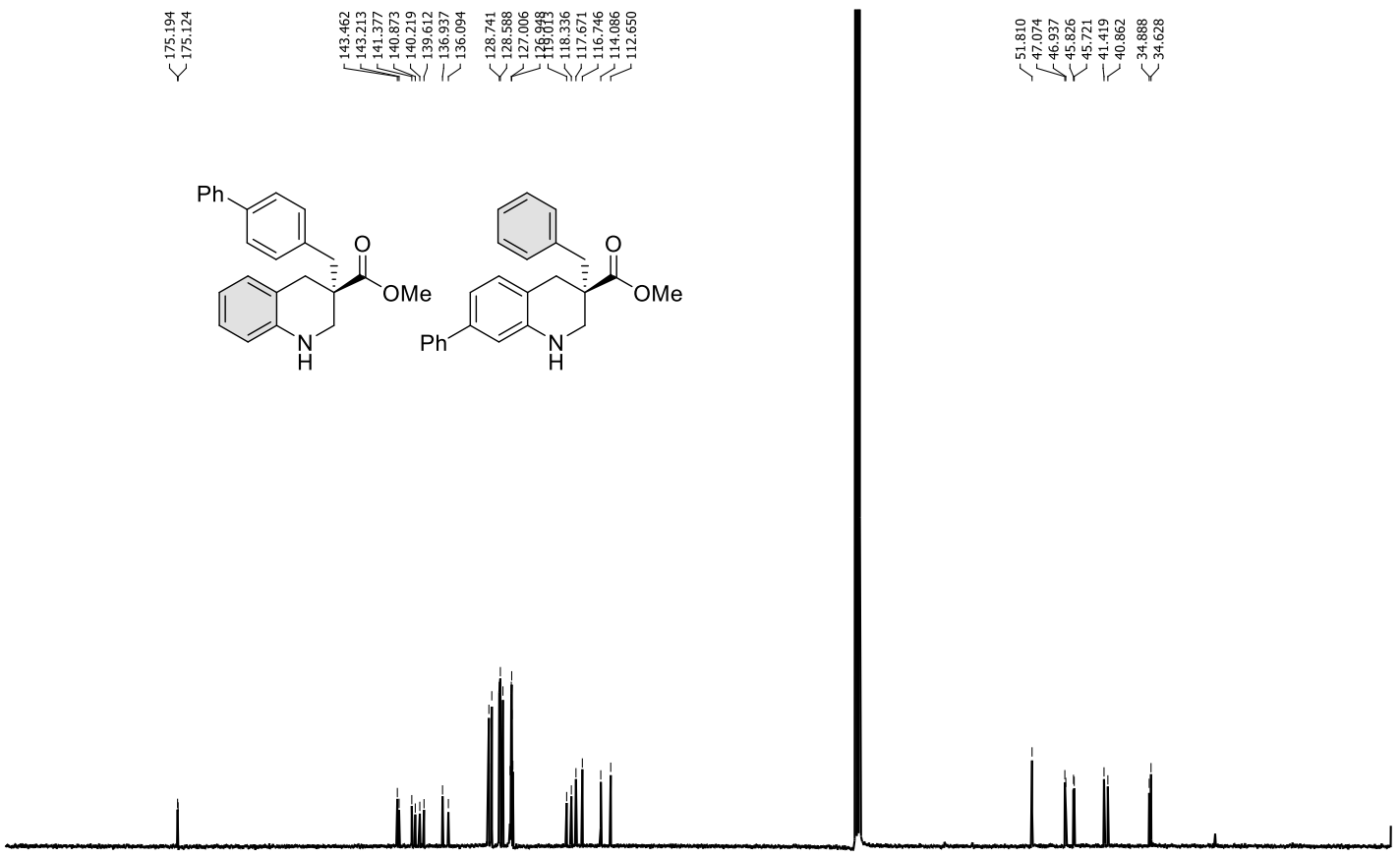

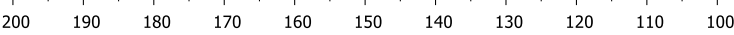

Page S87 of S104 
Mixture of methyl (R)-3-(2-methylbenzyl)-1,2,3,4-tetrahydroquinoline-3-carboxylate (6c-A) and methyl (R)3-benzyl-5-methyl-1,2,3,4-tetrahydroquinoline-3-carboxylate (6c-B)

${ }^{1} \mathrm{H}$ NMR (400 MHz, $\mathrm{CDCl}_{3}$ )

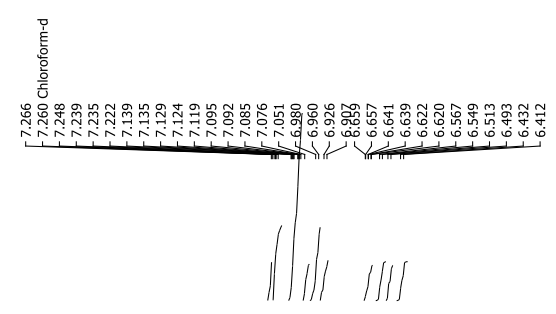

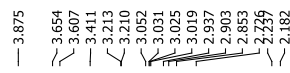
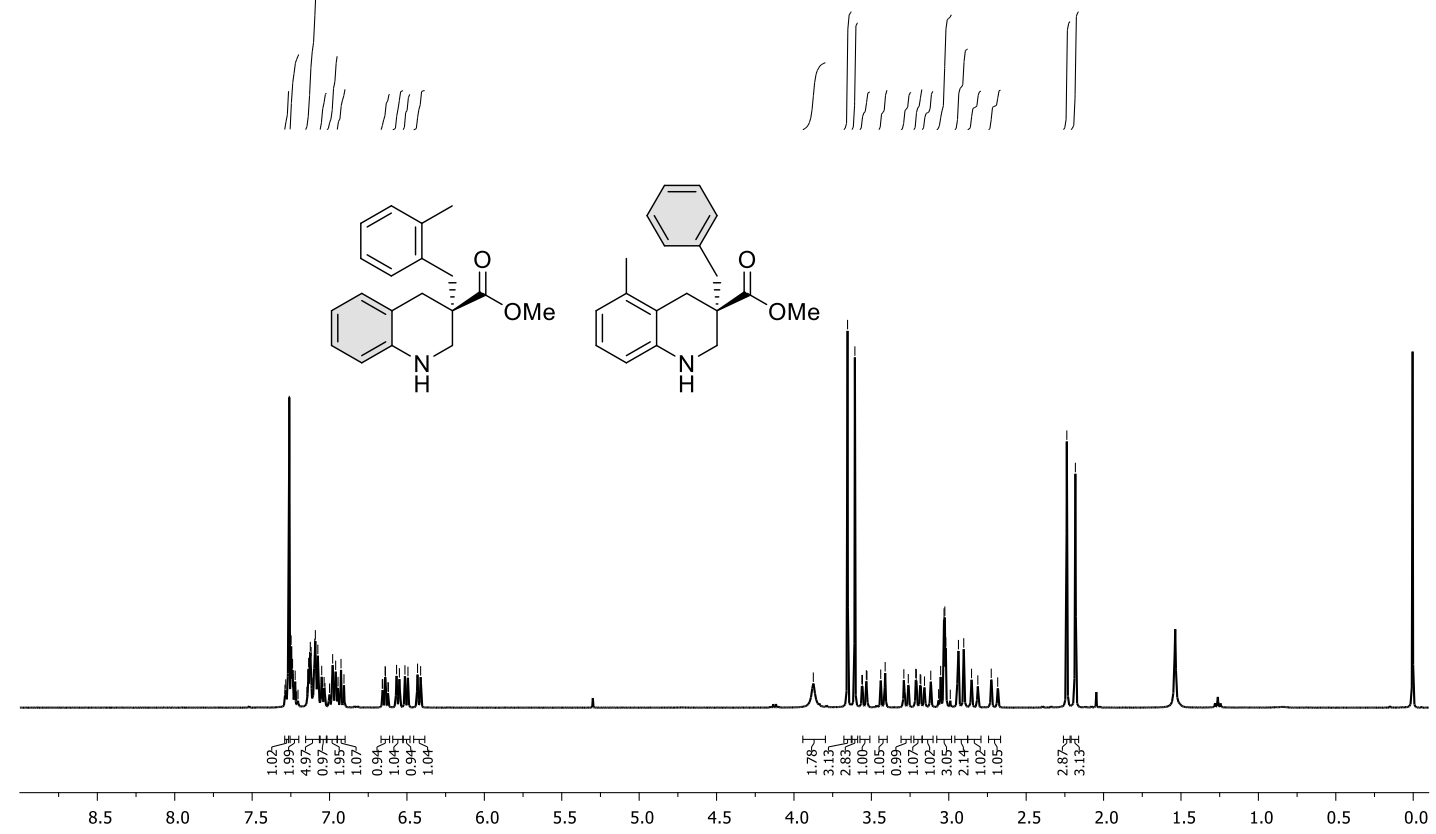

${ }^{13} \mathrm{C}$ NMR (101 MHz, $\left.\mathrm{CDCl}_{3}\right)$

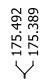

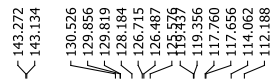

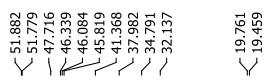<smiles>COC(=O)C1CNc2ccccc2C1</smiles><smiles>COC(=O)C1(Cc2ccccc2)CNc2cccc(C)c2C1</smiles>
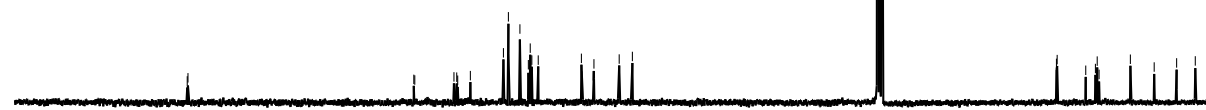
Mixture of methyl (R)-7-methyl-3-(2-methylbenzyl)-1,2,3,4-tetrahydroquinoline-3-carboxylate (6d-A) and methyl $(R)-5$-methyl-3-(4-methylbenzyl)-1,2,3,4-tetrahydroquinoline-3-carboxylate (6d-B)

${ }^{1} \mathrm{H}$ NMR (400 $\mathrm{MHz}, \mathrm{CDCl}_{3}$ )
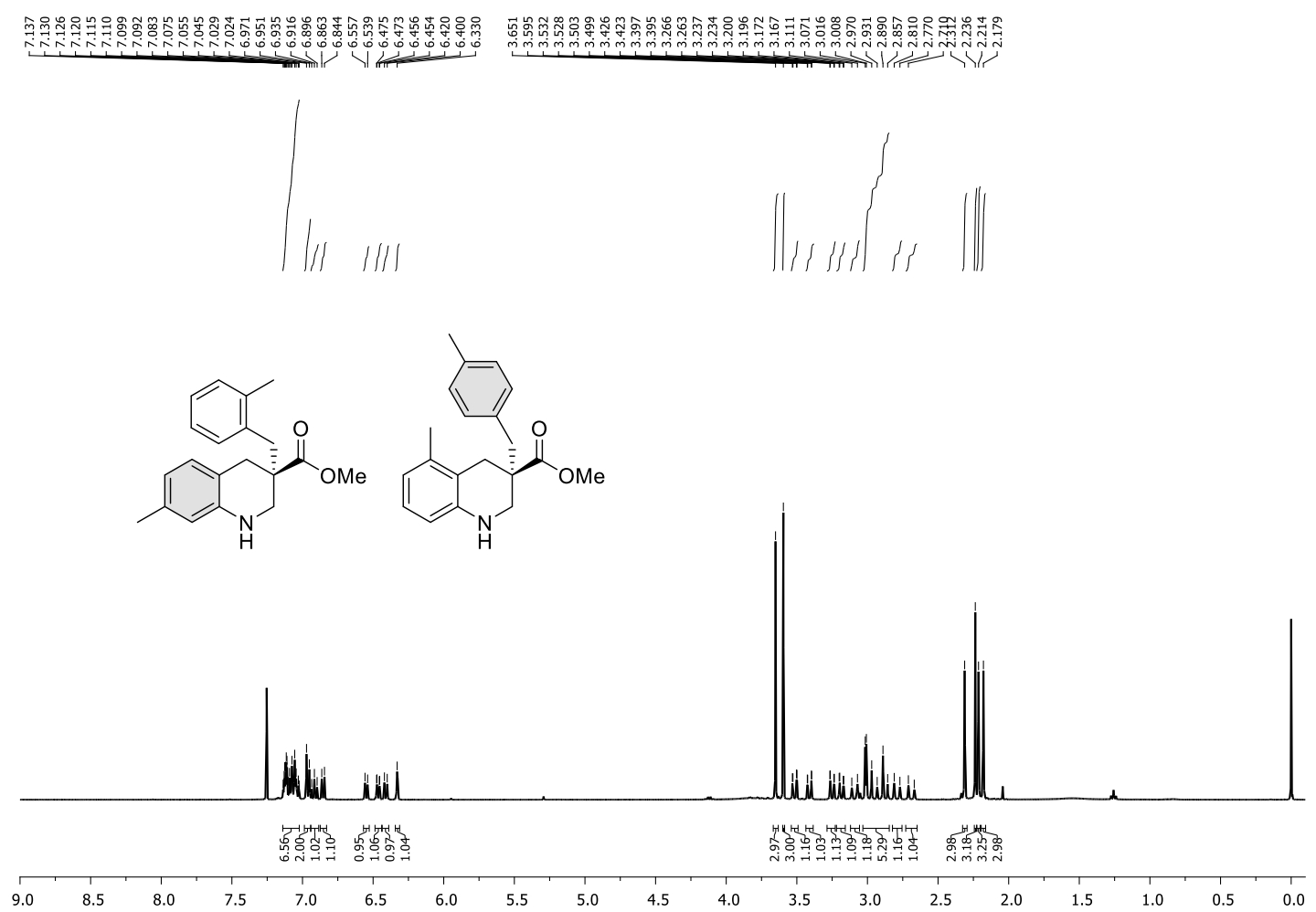

${ }^{13} \mathrm{C}$ NMR (101 MHz, $\left.\mathrm{CDCl}_{3}\right)$
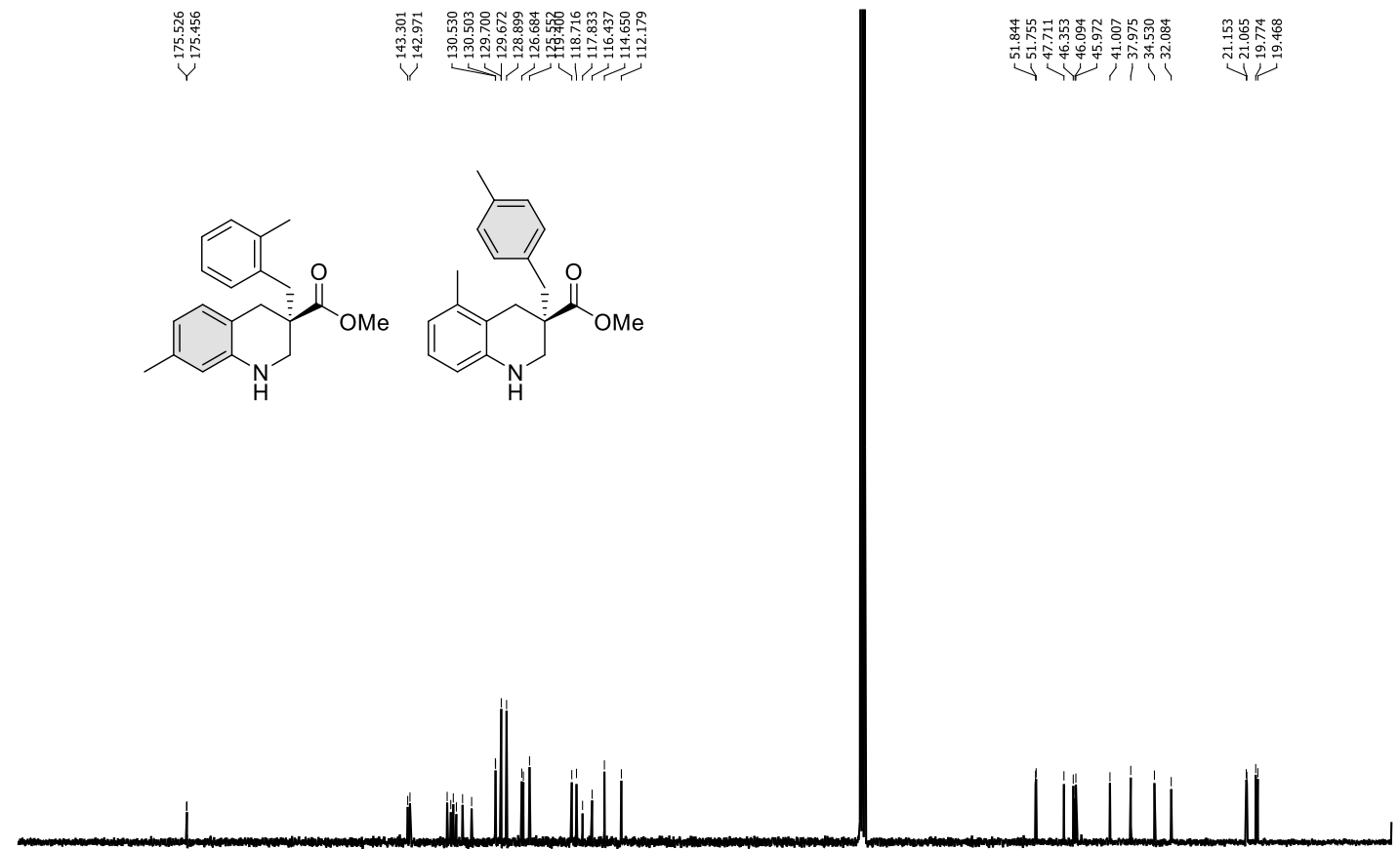

200

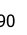

180

$170 \quad 160$

140

$130 \quad 120$

100

Page S89 of S104 
Mixture of methyl (R)-3-(4-bromobenzyl)-7-chloro-1,2,3,4-tetrahydroquinoline-3-carboxylate (6e-A) and methyl $(R)$-7-bromo-3-(4-chlorobenzyl)-1,2,3,4-tetrahydroquinoline-3-carboxylate (6e-B)

${ }^{1} \mathrm{H}$ NMR (400 MHz, $\mathrm{CDCl}_{3}$ )

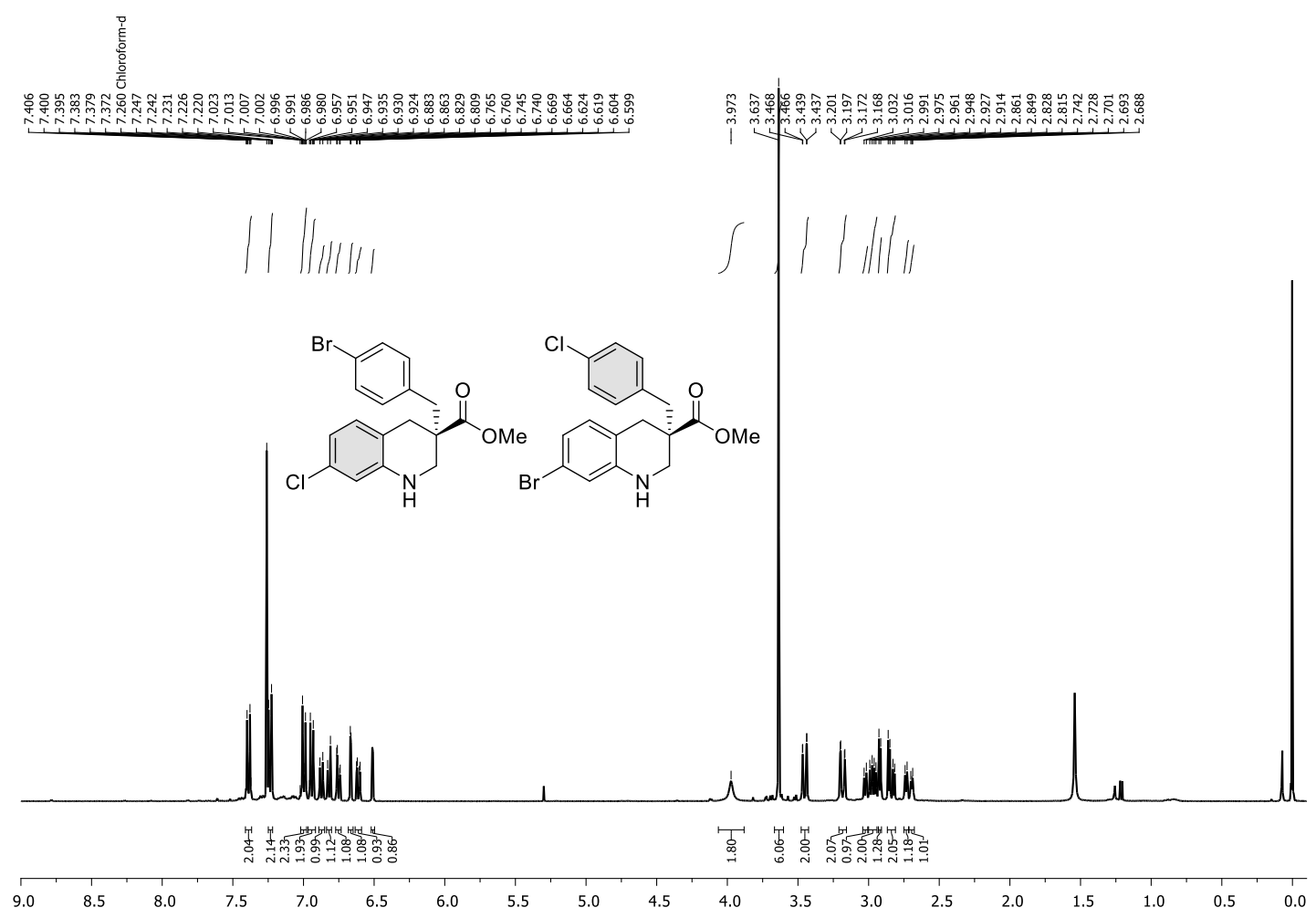

${ }^{13} \mathrm{C}$ NMR (101 MHz, $\left.\mathrm{CDCl}_{3}\right)$

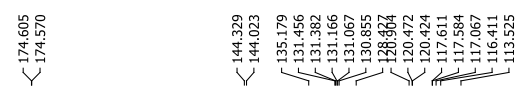<smiles>COC(=O)C1CNc2cc(Br)ccc2C1Cc1ccc(Cl)cc1</smiles>

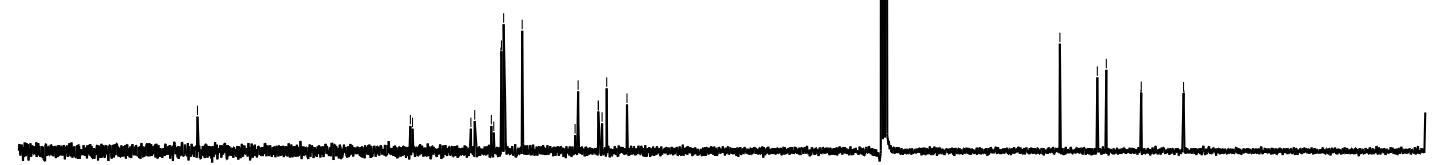

200 150 140 $130 \quad 120$ 100 $\begin{array}{llll}90 & 80 & 70 & 60\end{array}$ 
Methyl (R)-3-(cyclohexylmethyl)-7-methyl-1,2,3,4-tetrahydroquinoline-3-carboxylate (9b-A) ${ }^{1} \mathrm{H}$ NMR (400 MHz, $\left.\mathrm{CDCl}_{3}\right)$

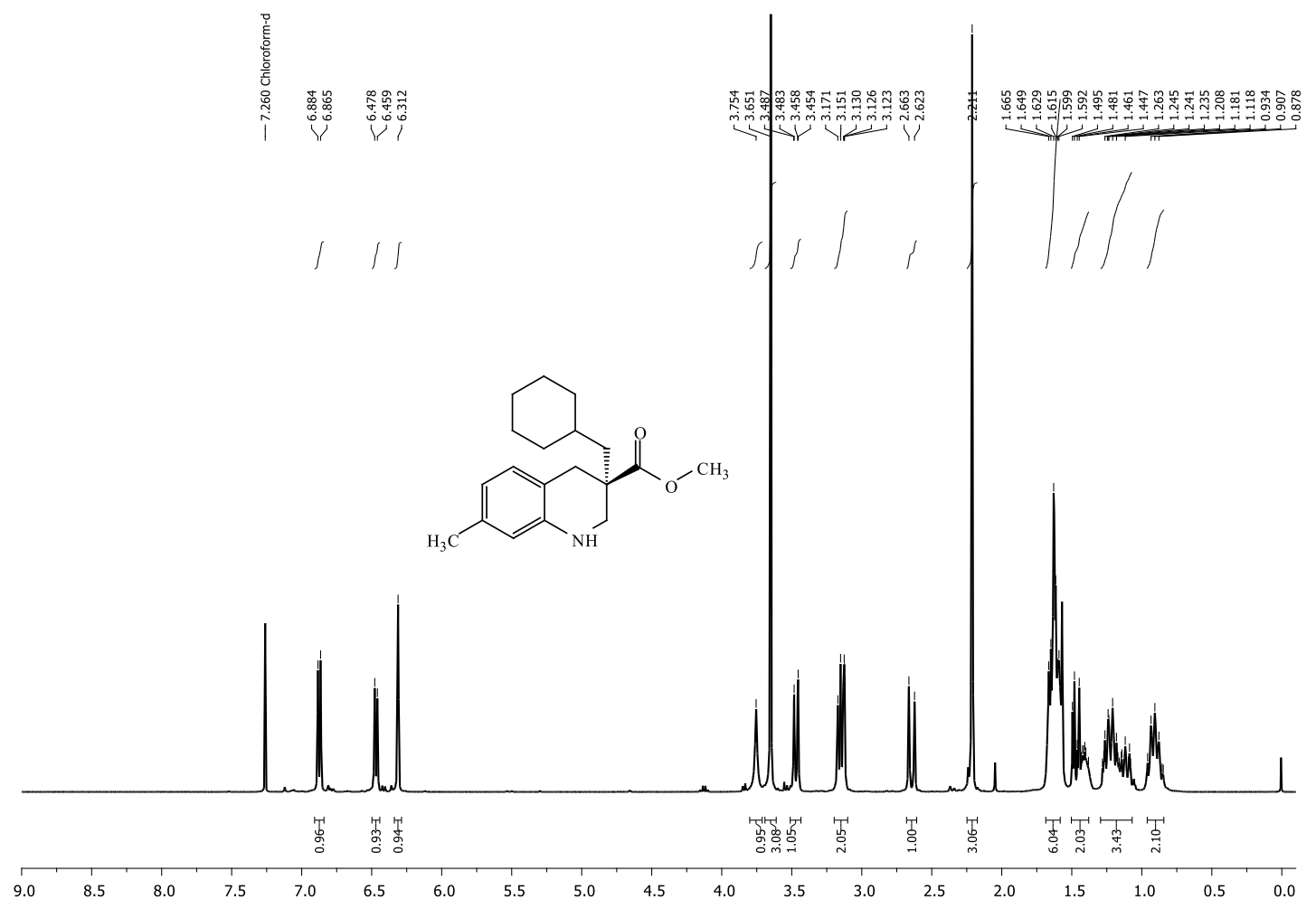

${ }^{13} \mathrm{C}$ NMR (101 MHz, $\left.\mathrm{CDCl}_{3}\right)$

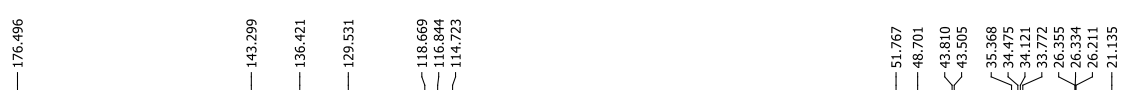<smiles>COC(=O)C1CNc2cc(C)ccc2C1</smiles>
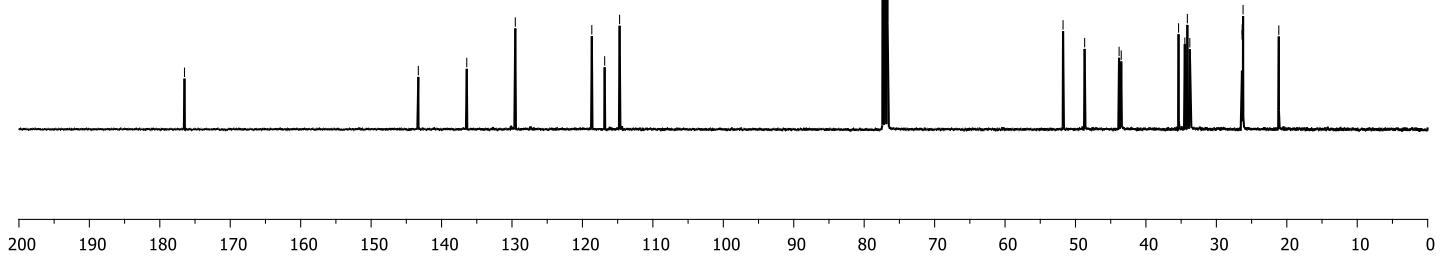
Methyl (R)-3-(cyclohexylmethyl)-5-methyl-1,2,3,4-tetrahydroquinoline-3-carboxylate (9c-A) ${ }^{1} \mathrm{H}$ NMR (400 MHz, $\mathrm{CDCl}_{3}$ )

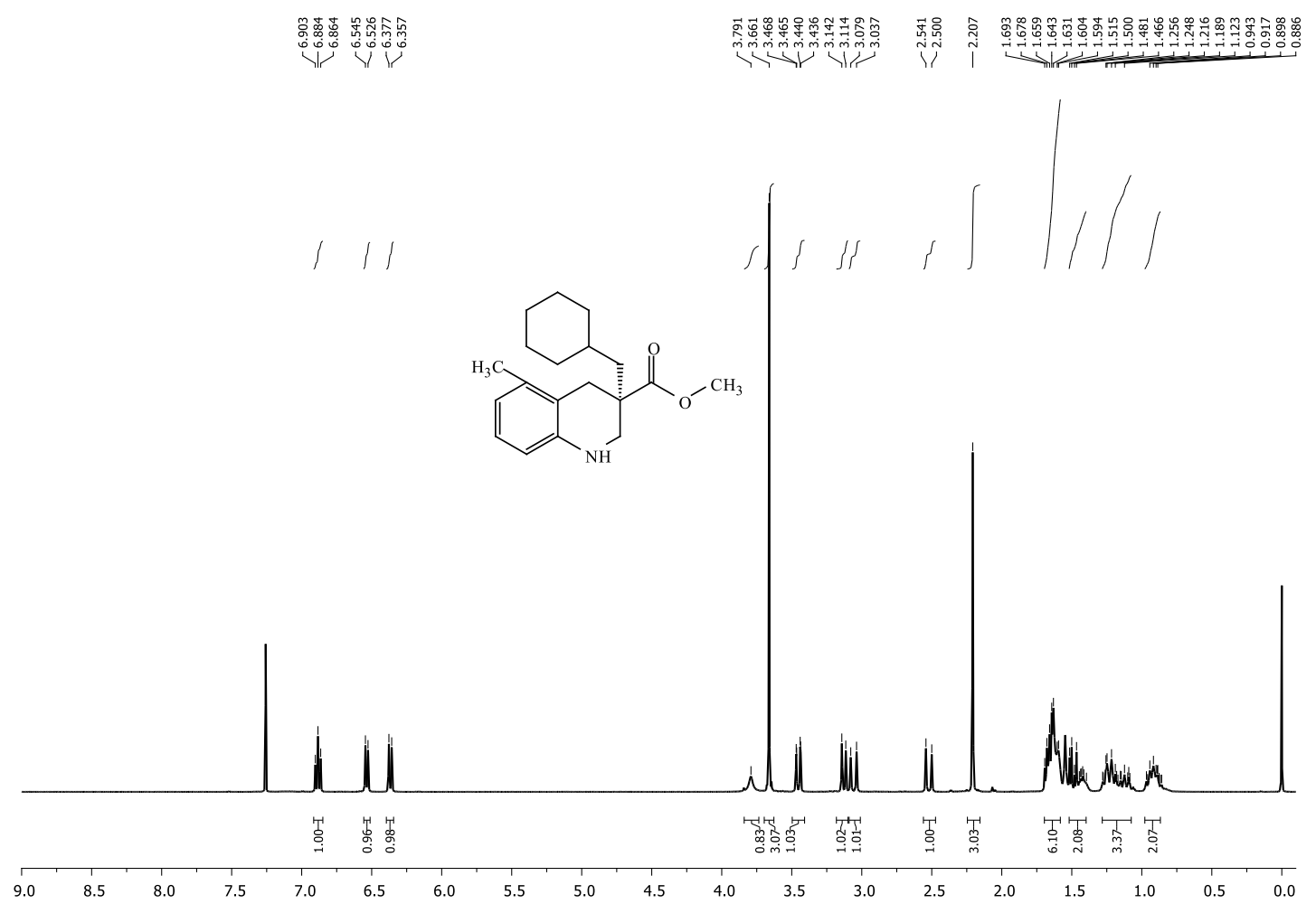

${ }^{13} \mathrm{C}$ NMR (101 MHz, $\left.\mathrm{CDCl}_{3}\right)$

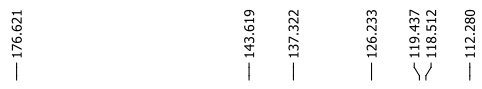

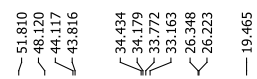<smiles>COC(=O)C1(CC2CCCCC2)CNc2cccc(C)c2C1</smiles>

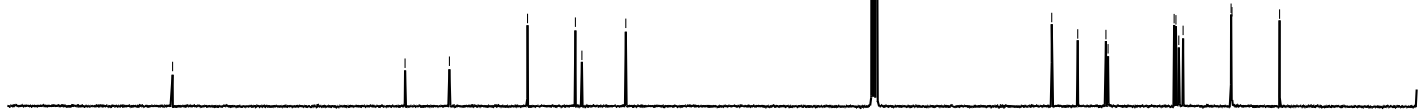


Methyl (R)-3-(cyclohexylmethyl)-7-phenyl-1,2,3,4-tetrahydroquinoline-3-carboxylate (9d-A) ${ }^{1} \mathrm{H}$ NMR (400 MHz, $\mathrm{CDCl}_{3}$ )

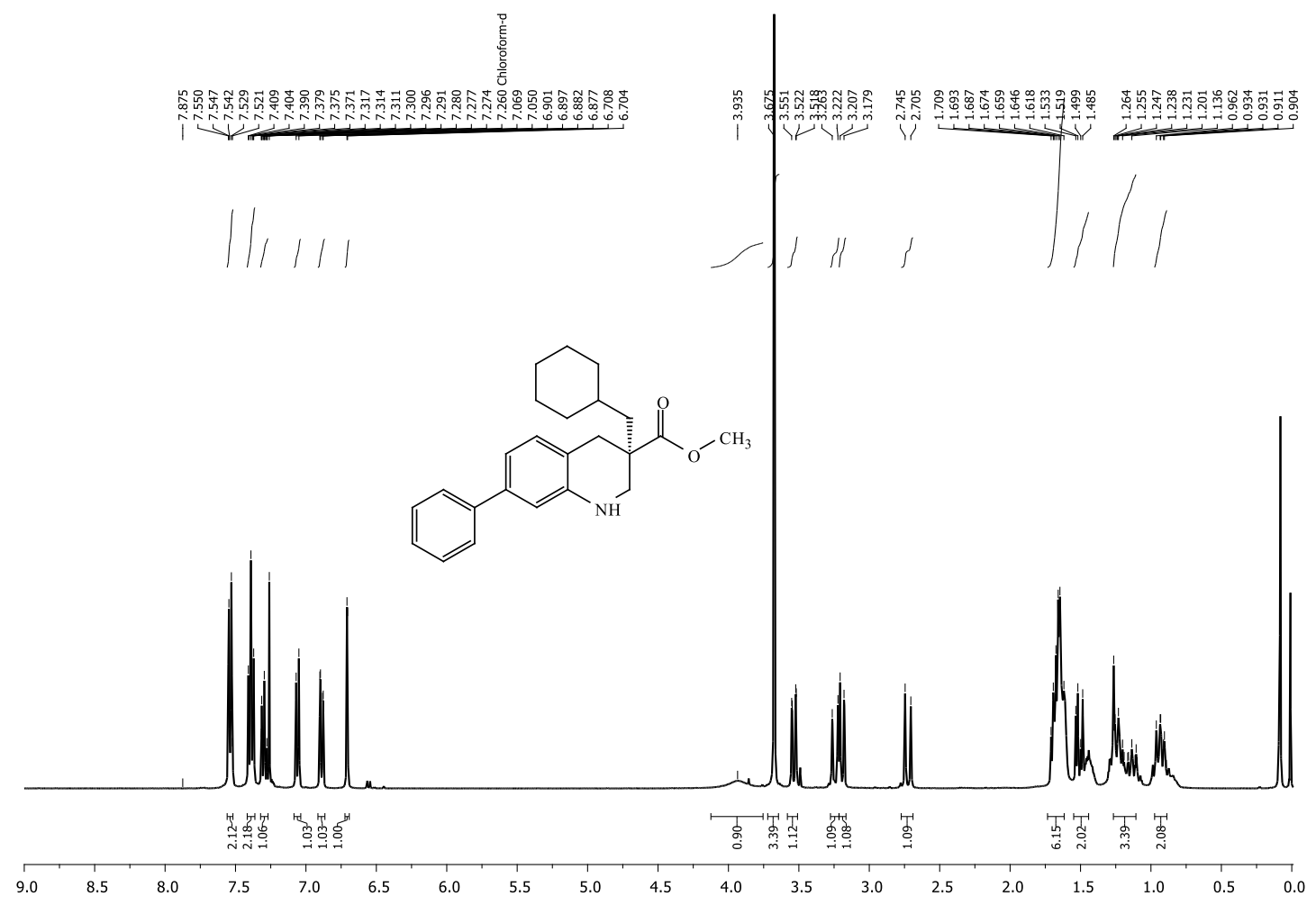

${ }^{13} \mathrm{C}$ NMR (101 MHz, $\left.\mathrm{CDCl}_{3}\right)$

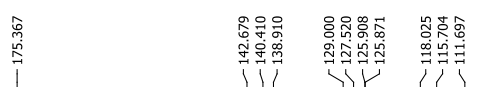
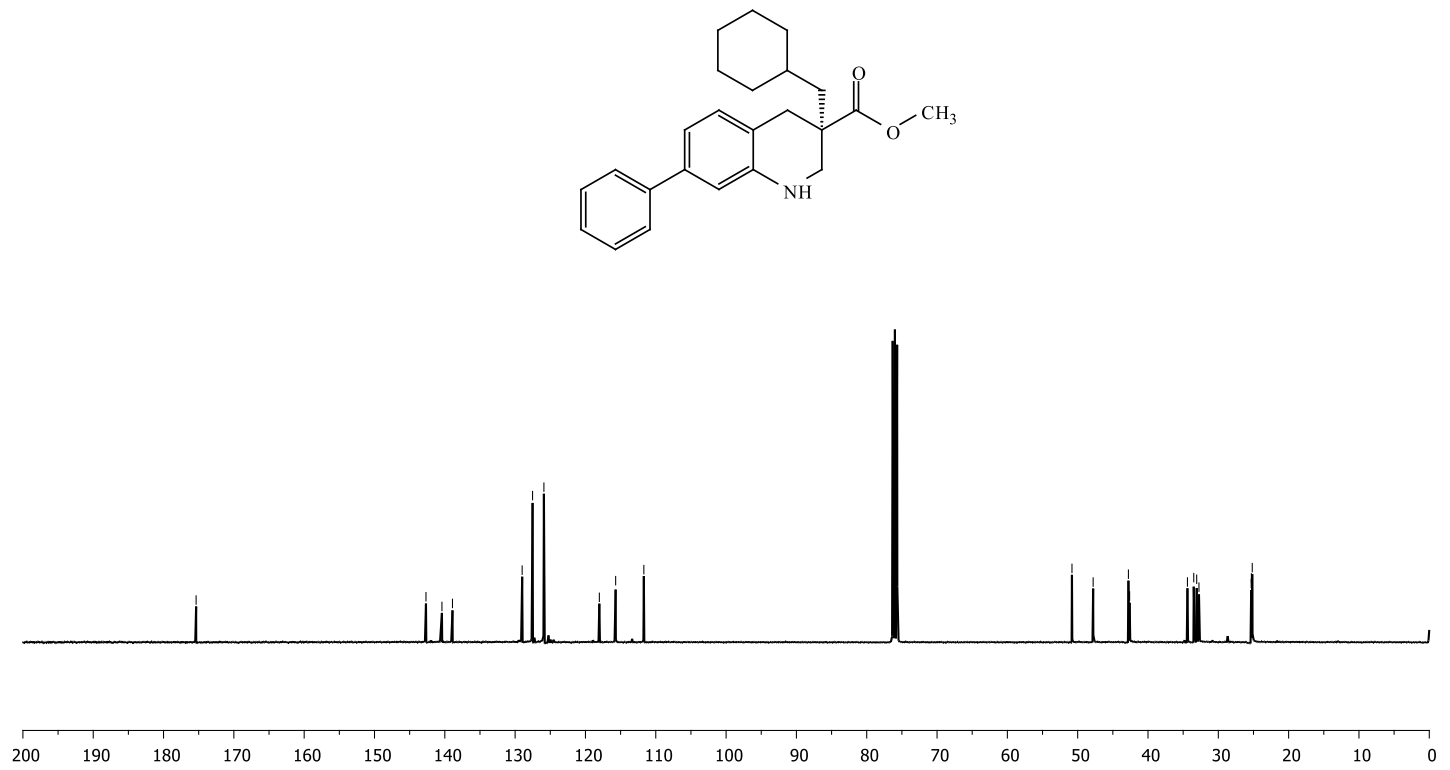
Methyl (R)-3-((tetrahydro-2H-pyran-4-yl)methyl)-1,2,3,4-tetrahydroquinoline-3-carboxylate (9e-A) ${ }^{1} \mathrm{H}$ NMR (400 MHz, $\left.\mathrm{CDCl}_{3}\right)$
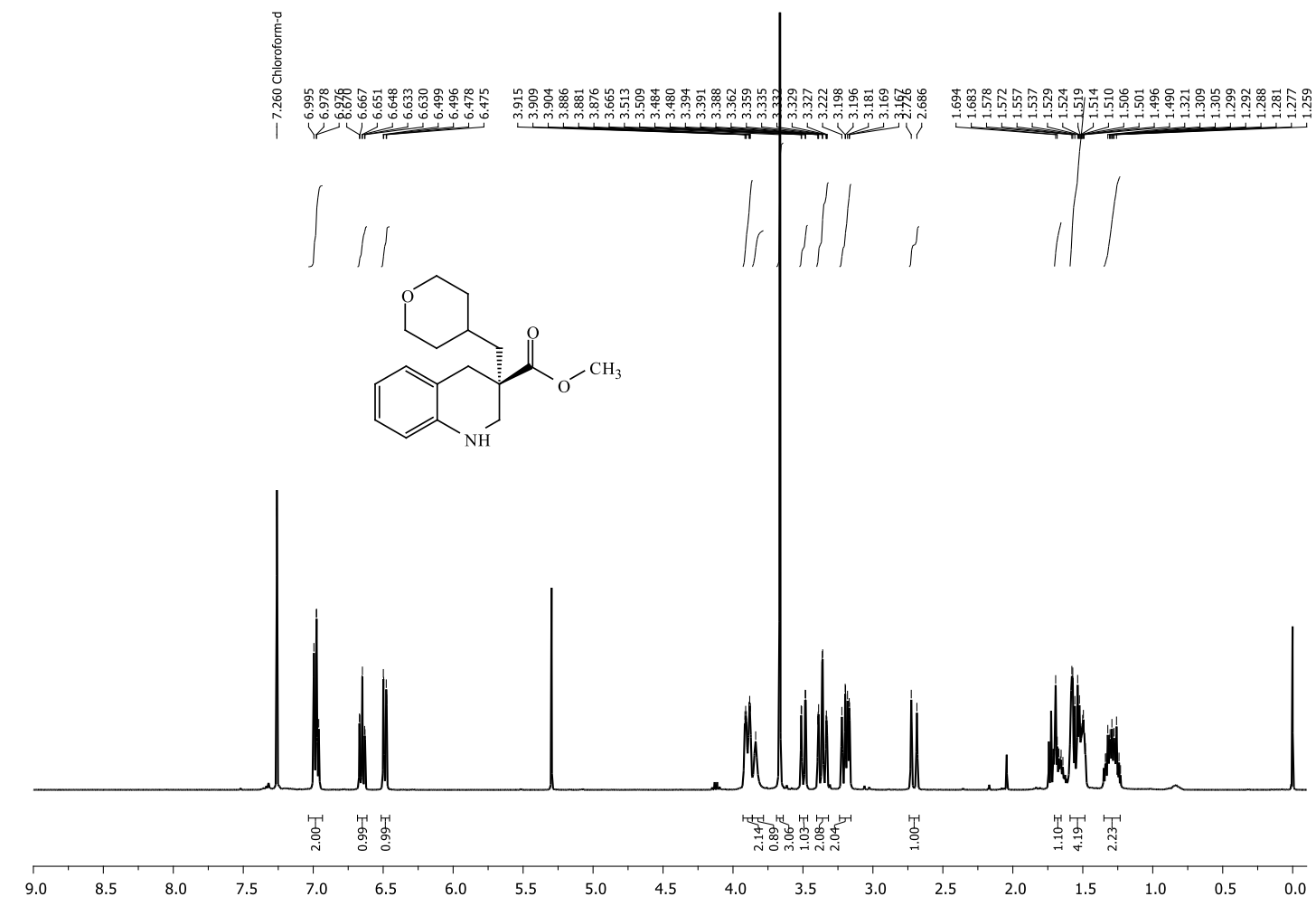

${ }^{13} \mathrm{C}$ NMR (101 MHz, $\left.\mathrm{CDCl}_{3}\right)$

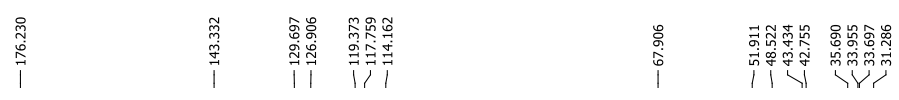<smiles>COC(=O)C1(CC2CCOCC2)CNc2ccccc2C1</smiles>

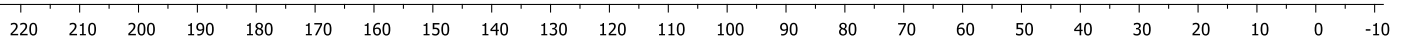


Methyl (R)-3-(4-nitrobenzyl)-1,2,3,4-tetrahydroquinoline-3-carboxylate (9f-A)

${ }^{1} \mathrm{H}$ NMR (400 MHz, $\mathrm{CDCl}_{3}$ )
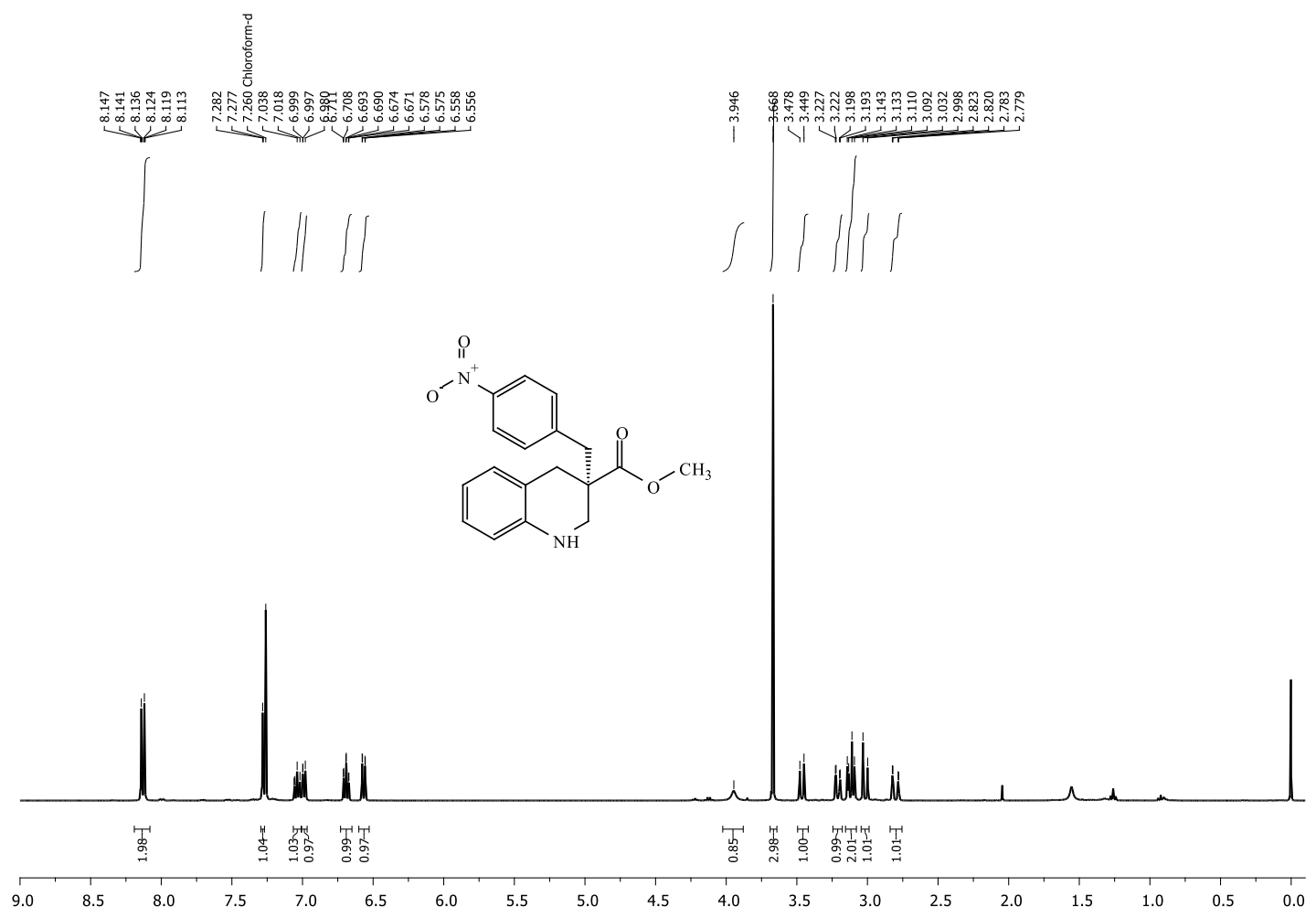

${ }^{13} \mathrm{C}$ NMR (101 MHz, $\left.\mathrm{CDCl}_{3}\right)$
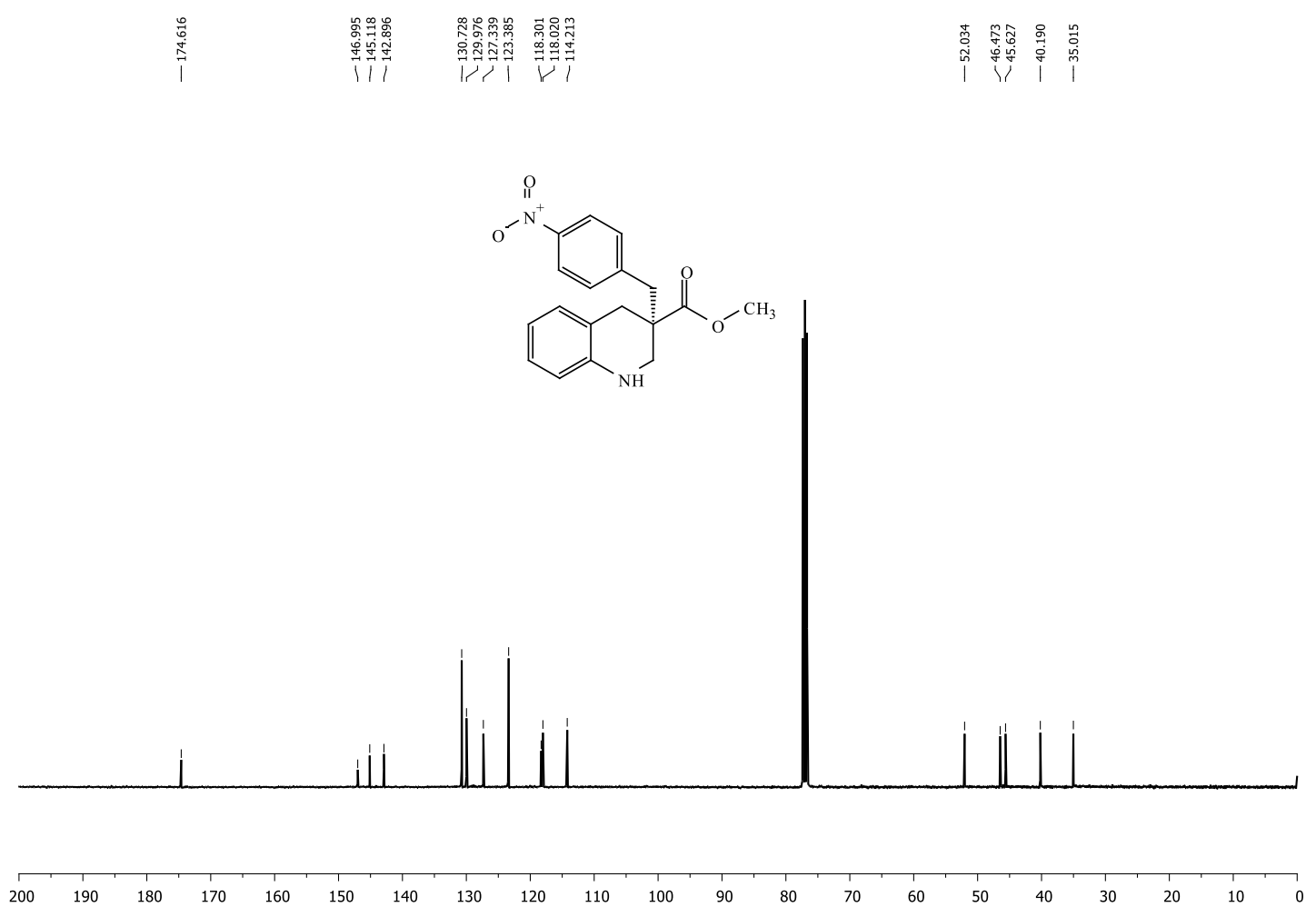
Methyl (R)-3-methyl-1,2,3,4-tetrahydroquinoline-3-carboxylate (9g-A)

${ }^{1} \mathrm{H}$ NMR (400 MHz, $\mathrm{CDCl}_{3}$ )

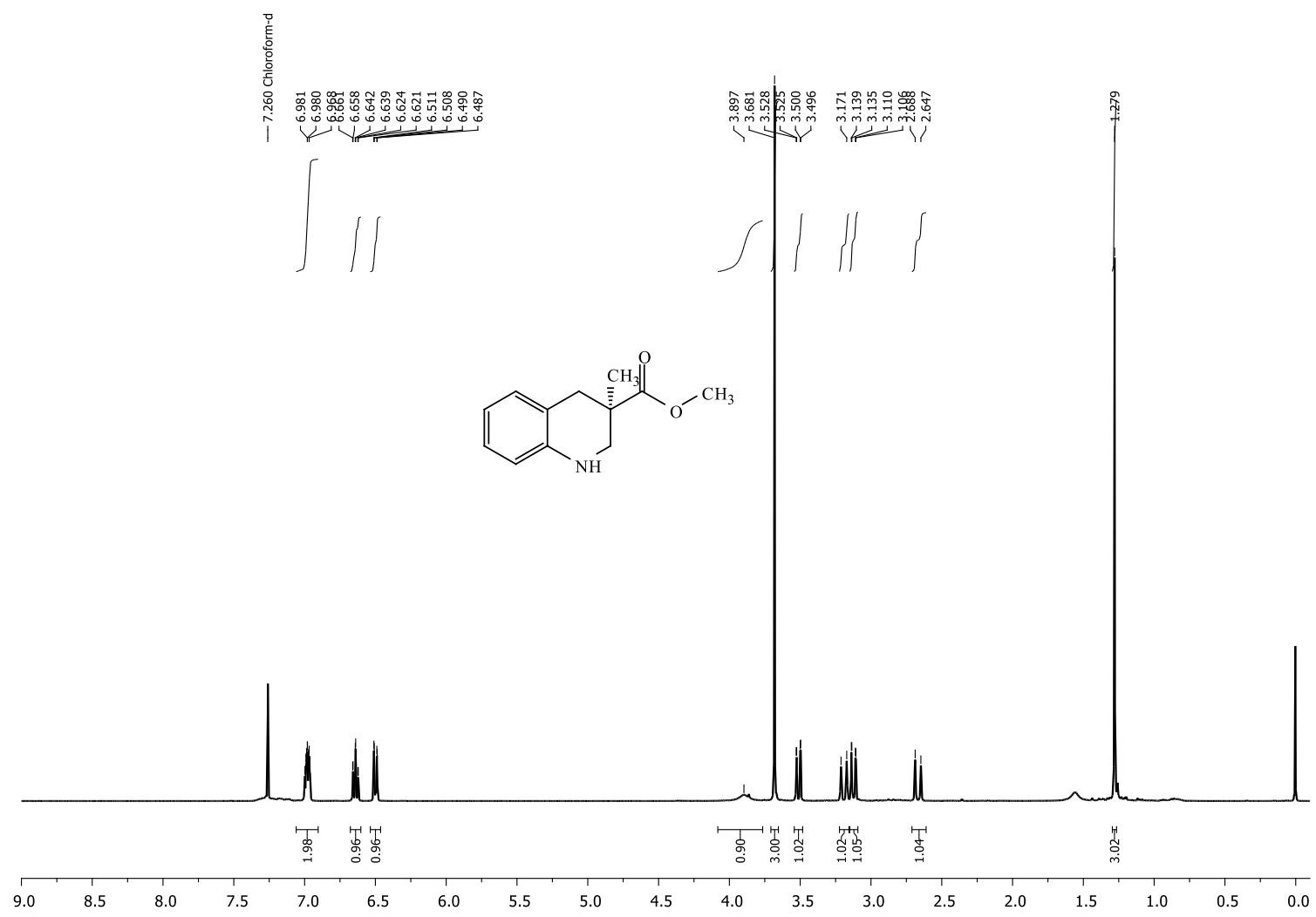

${ }^{13} \mathrm{C}$ NMR (101 MHz, $\left.\mathrm{CDCl}_{3}\right)$

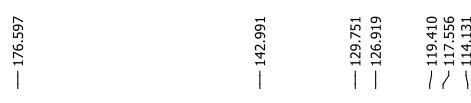
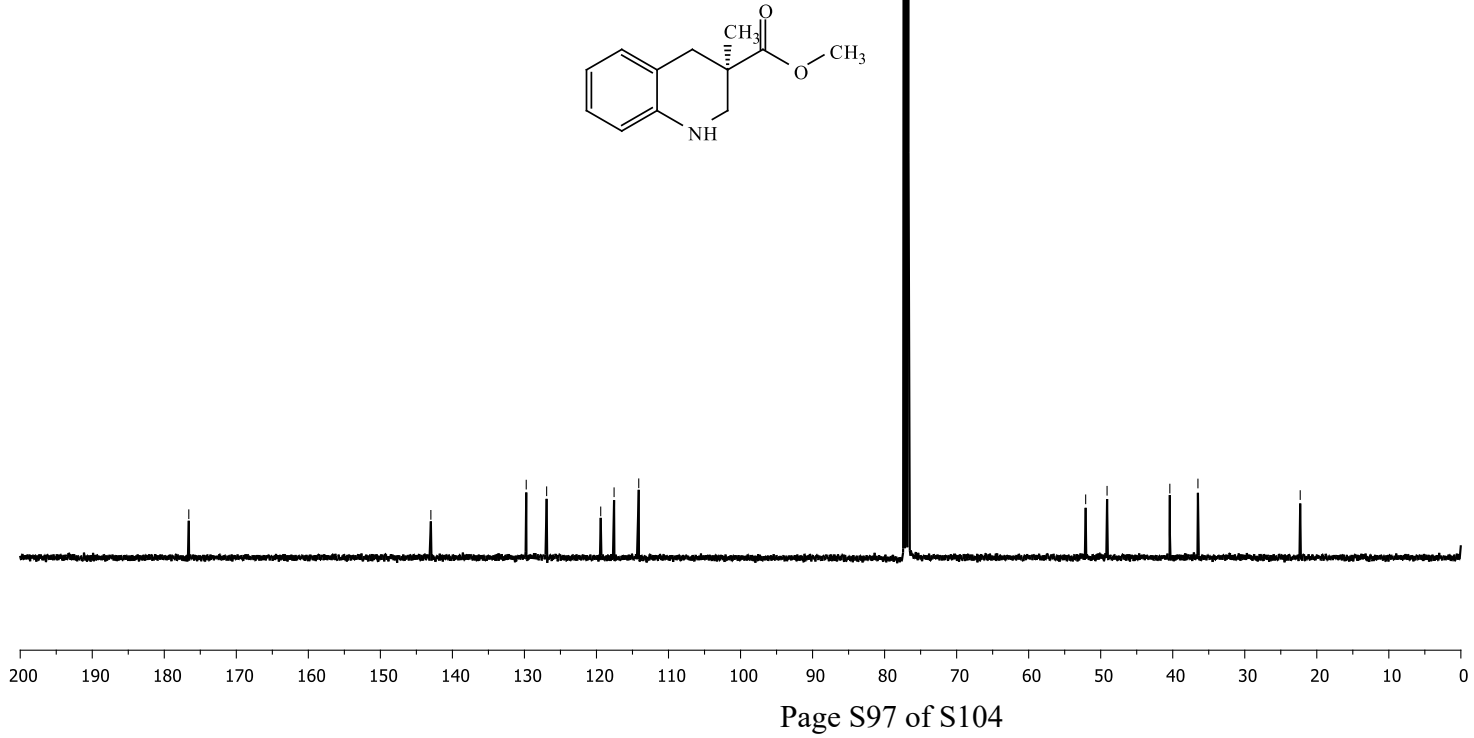
(S)-3-Amino-2-benzyl-2-(cyclohexylmethyl)propanoic acid (10) ${ }^{1} \mathrm{H}$ NMR (400 MHz, $\left.\mathrm{CD}_{3} \mathrm{OD}\right)$

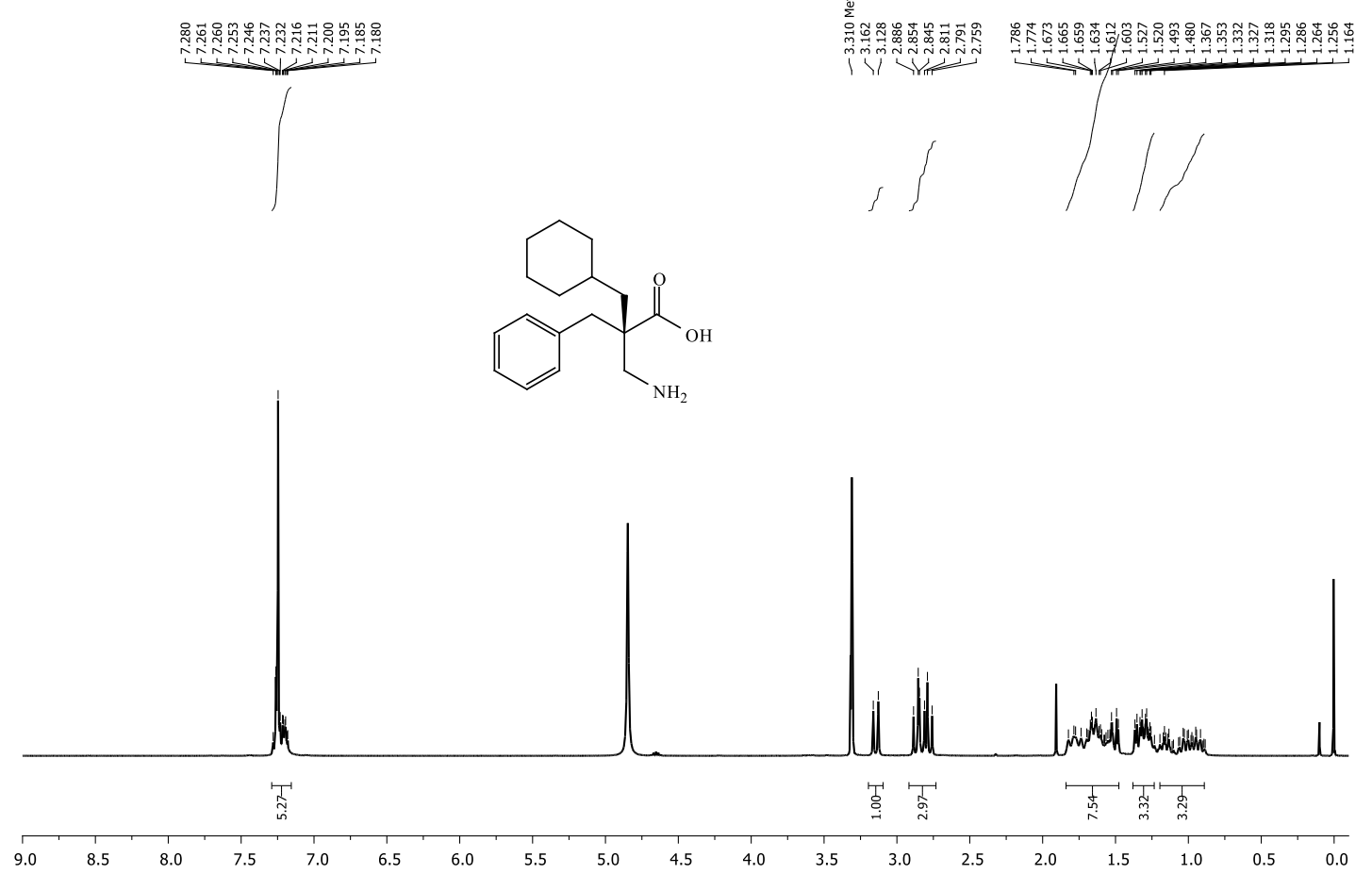

${ }^{13} \mathrm{C}$ NMR (101 MHz, CD 3 OD)

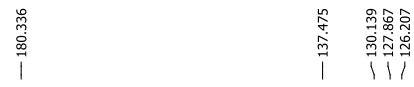

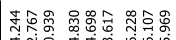

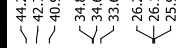
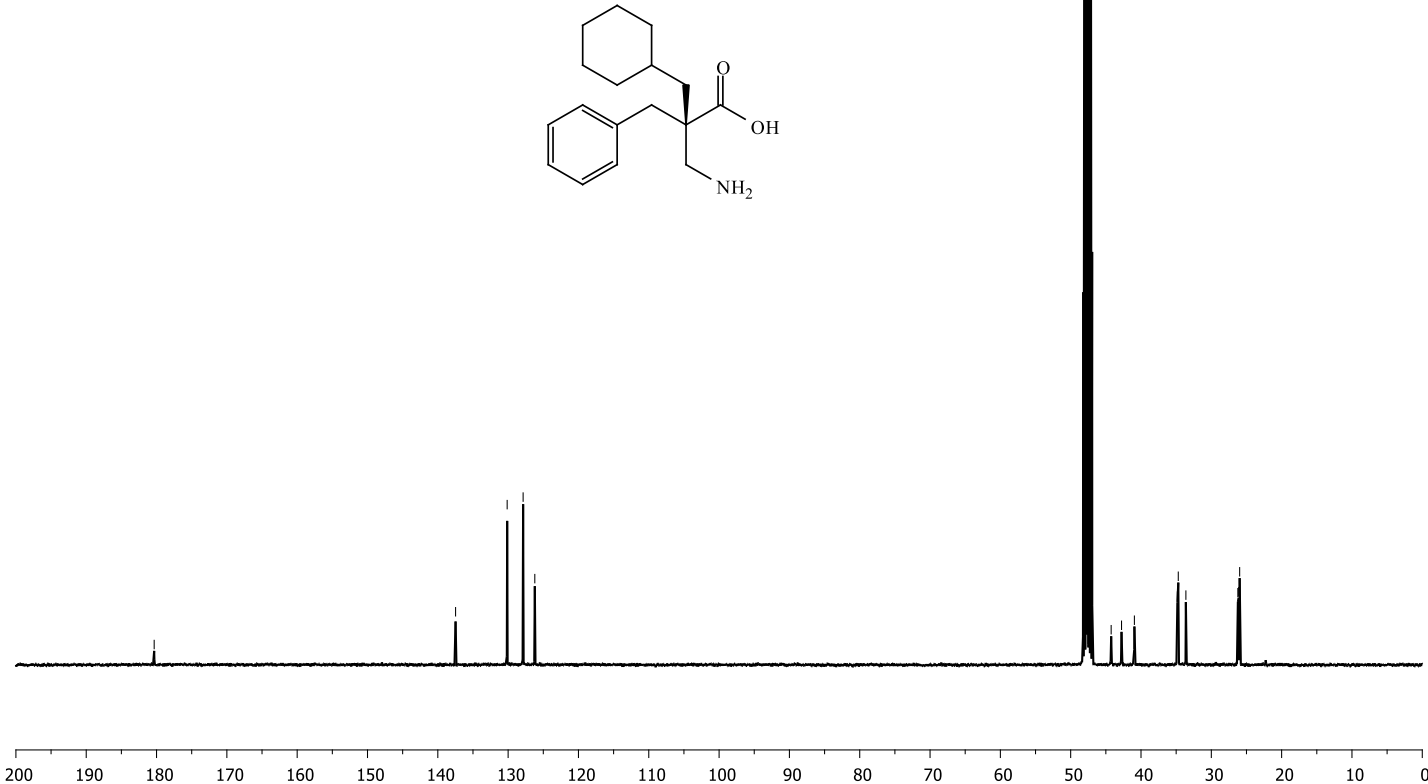

Page S98 of S104 
Methyl 2-benzyl-2-methyl-3-((3-methylquinolin-6-yl)amino)propanoate (methyl ester of S12) ${ }^{1} \mathrm{H}$ NMR (600 MHz, $\left.\mathrm{CD}_{3} \mathrm{OD}\right)$

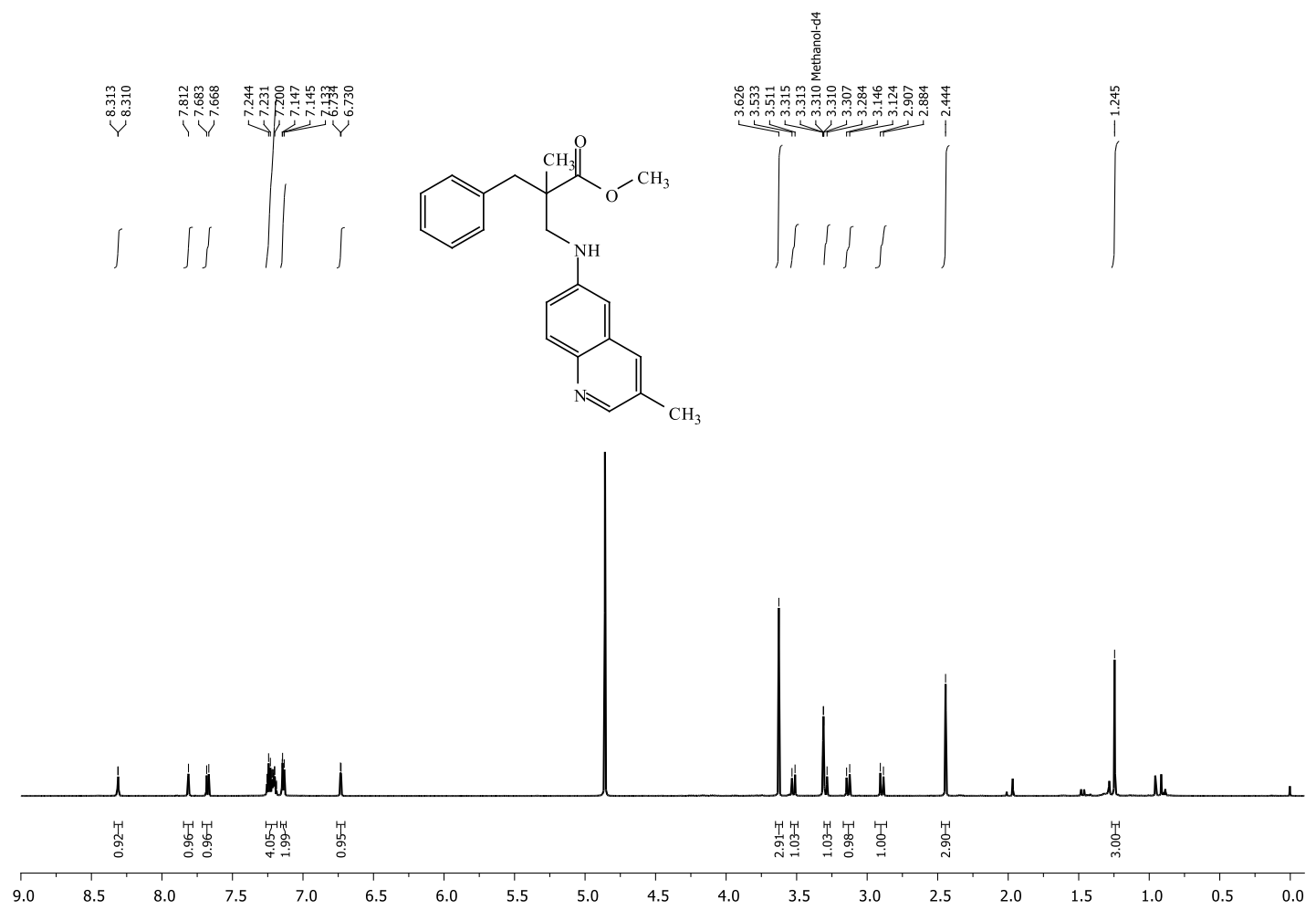

${ }^{13} \mathrm{C}$ NMR (151 MHz, CD 3 OD)
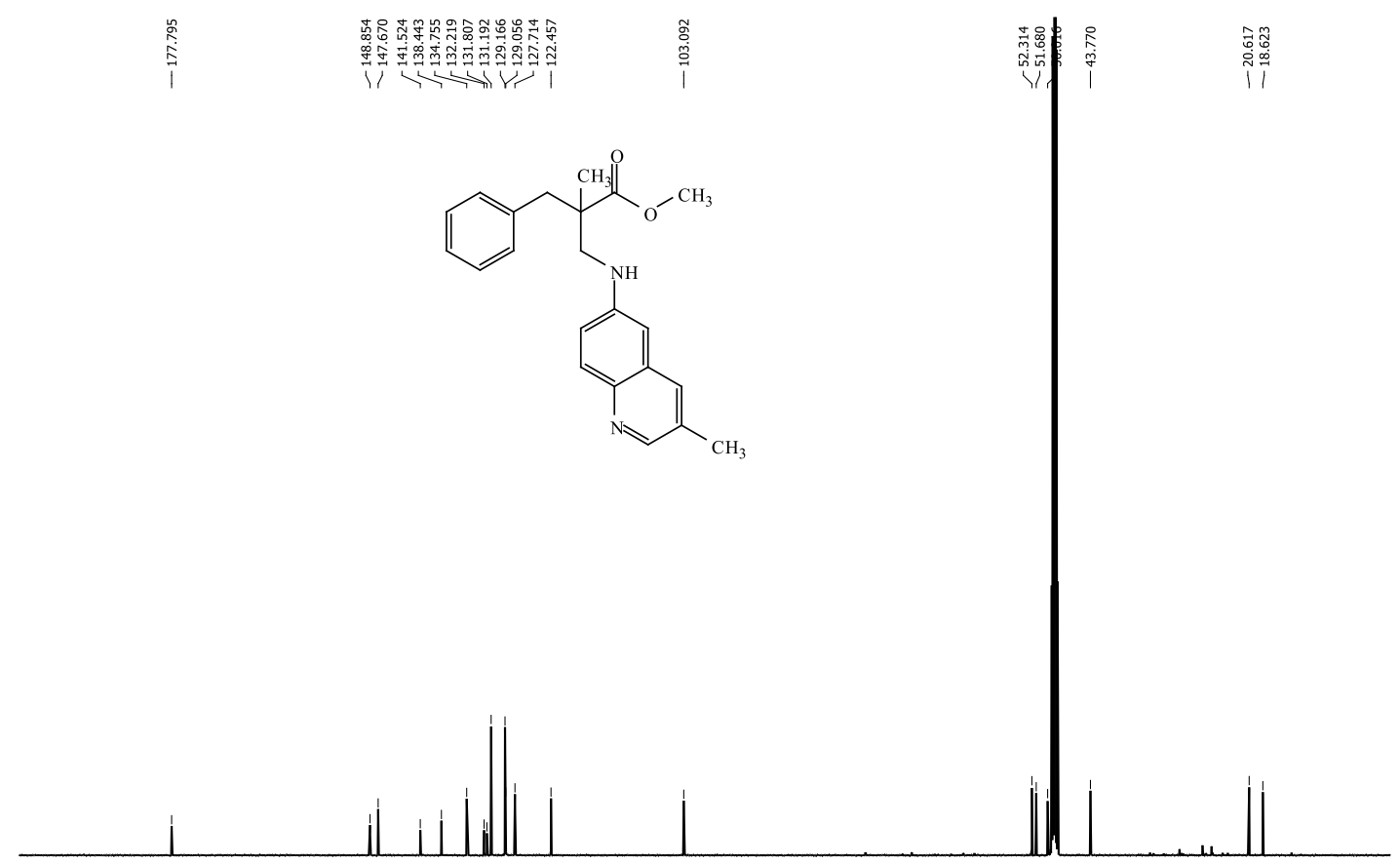

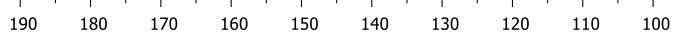

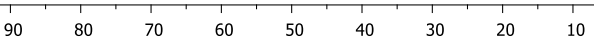


DEPT 135 (151 MHz, CD ${ }_{3}$ OD)
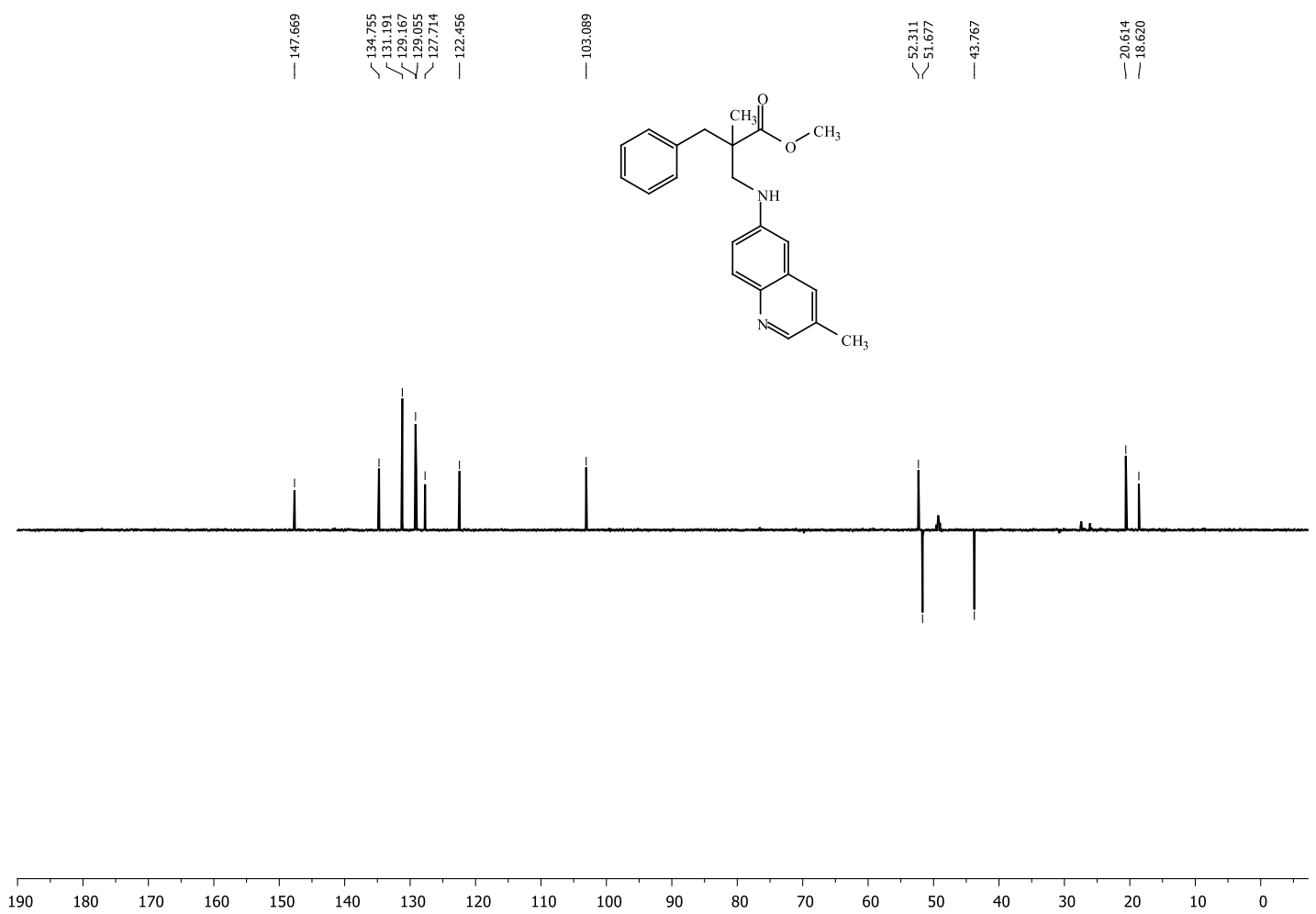

\section{$\operatorname{COSY}\left(600 \mathrm{MHz}, \mathrm{CD}_{3} \mathrm{OD}\right)$}

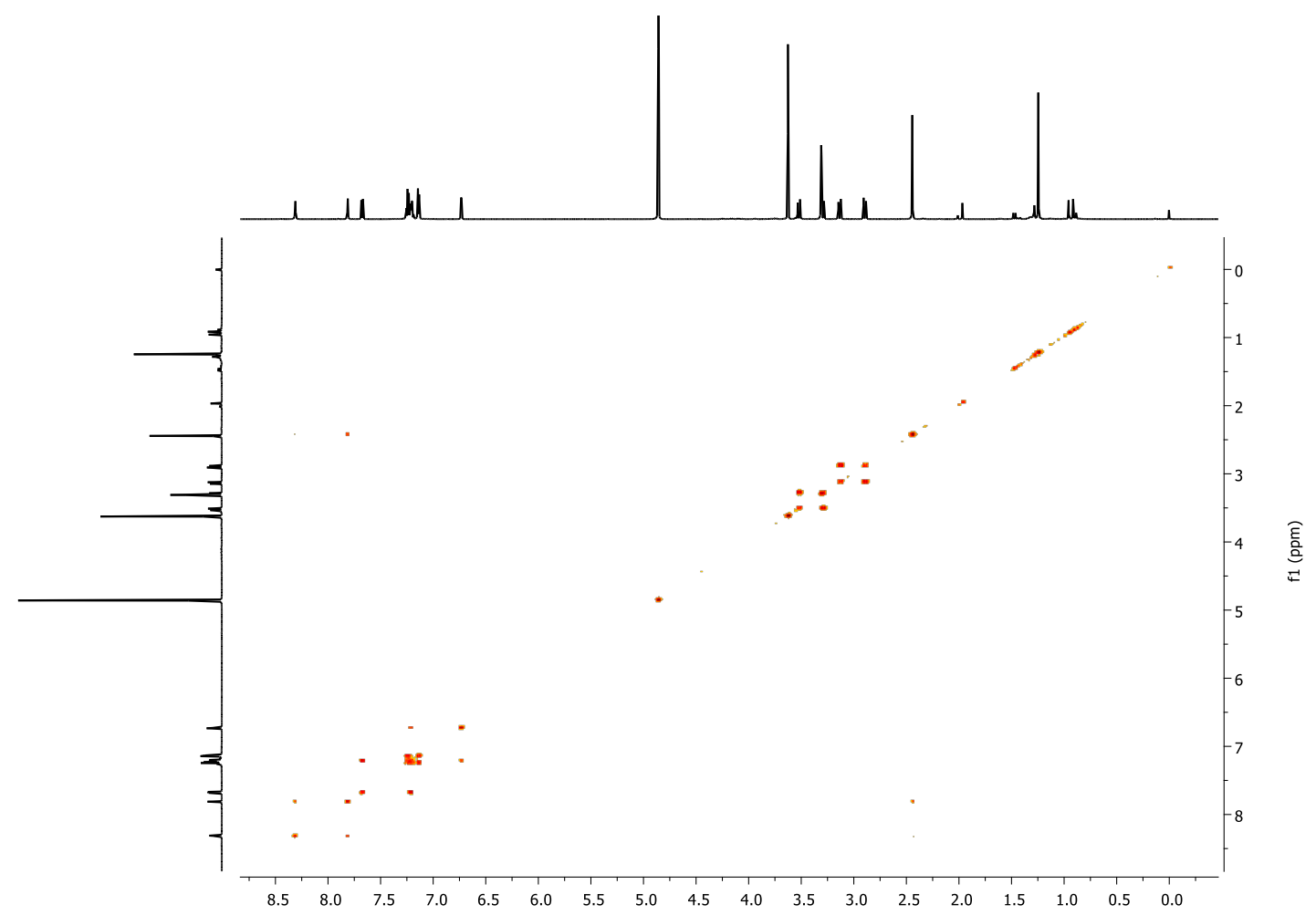


HMQC (600 MHz, CD ${ }_{3}$ OD)

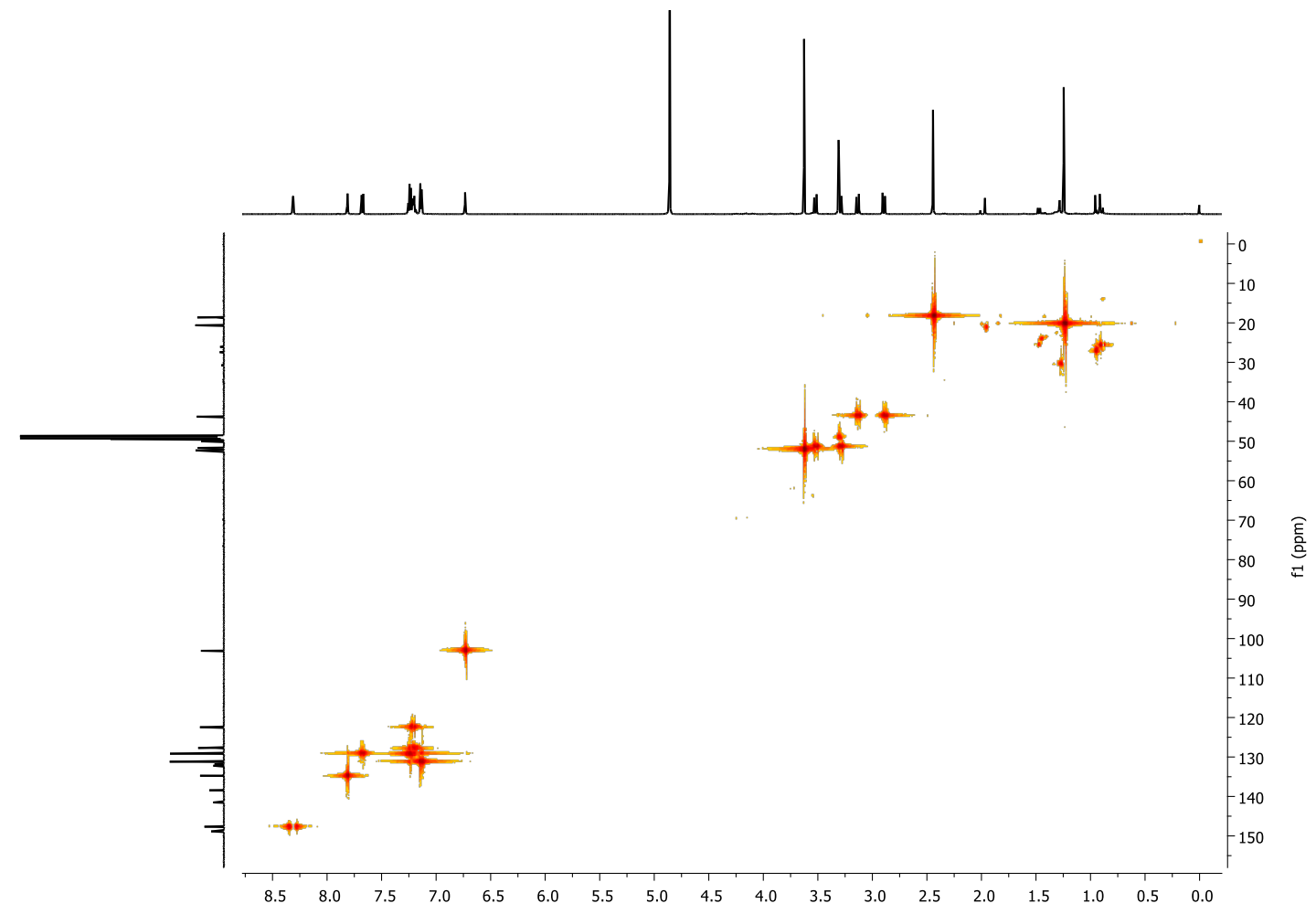

HMBC (600 MHz, CD 3 OD)

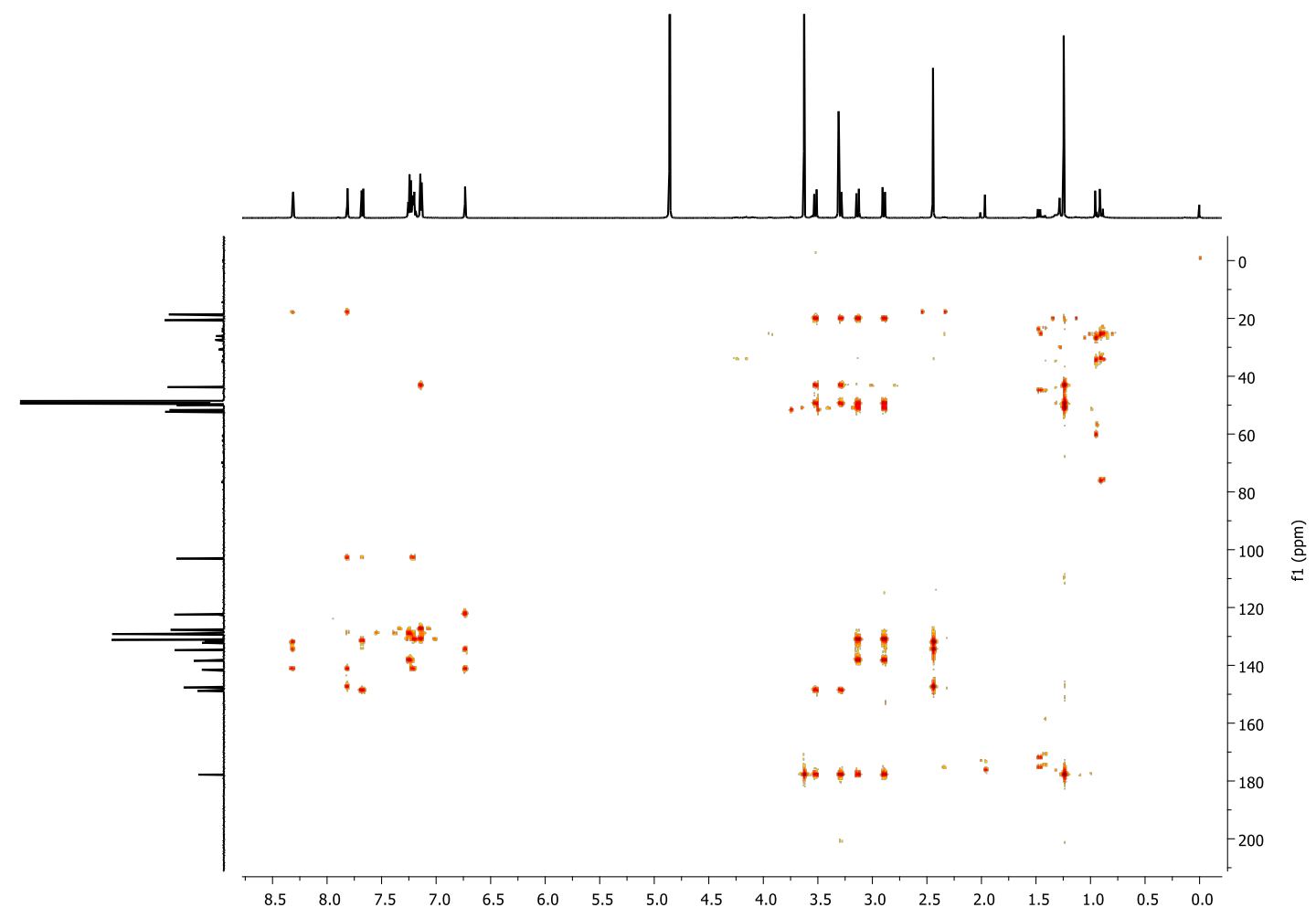


Methyl 6-(3,5-di-tert-butyl-1-methyl-4-oxocyclohexa-2,5-dien-1-yl)-1-(3,5-di-tert-butyl-4-hydroxybenzyl)-3methyl-1,2,3,4-tetrahydroquinoline-3-carboxylate (S13)

${ }^{1} \mathrm{H}$ NMR (400 MHz, $\mathrm{CDCl}_{3}$ )

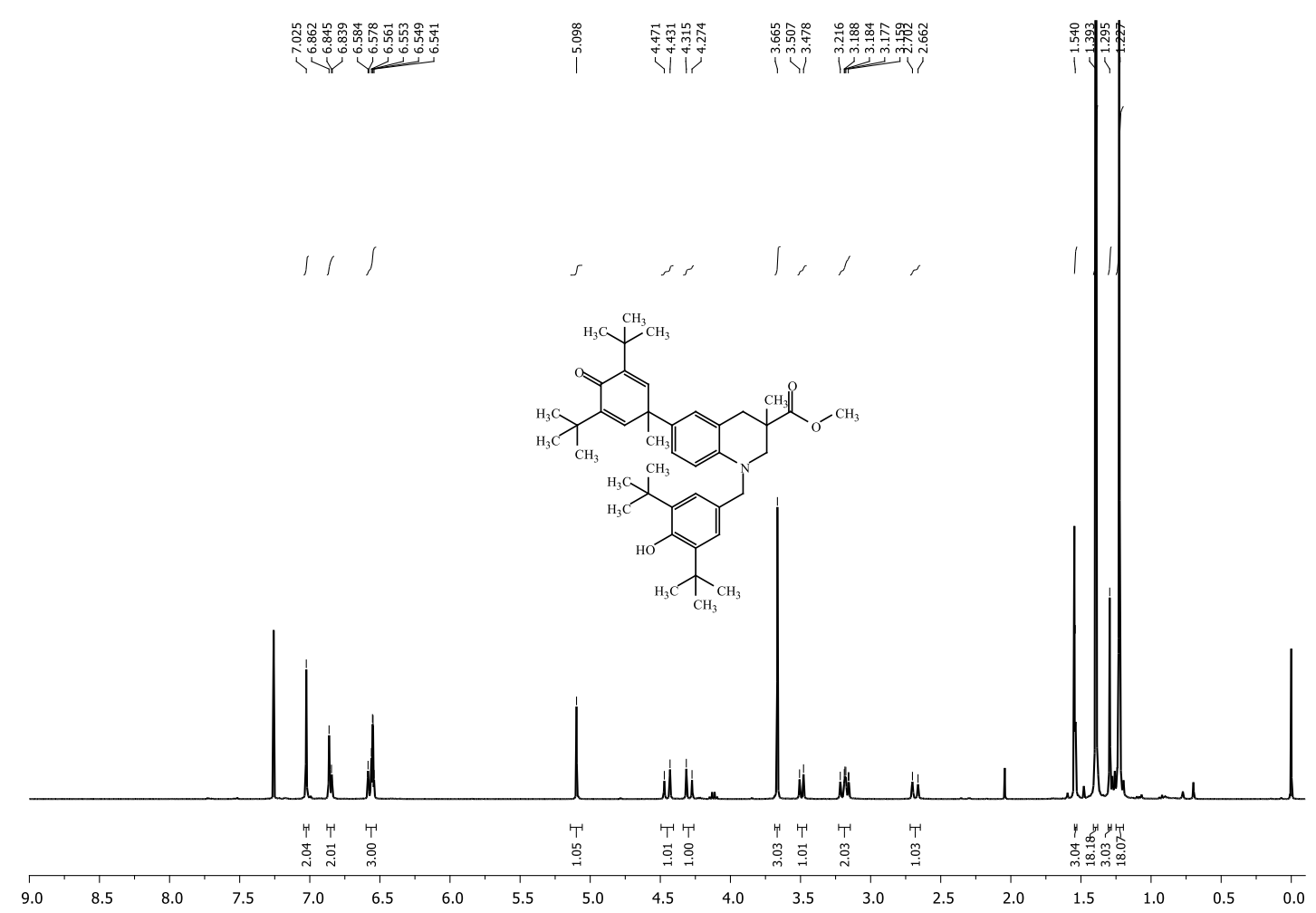

${ }^{13} \mathrm{C}$ NMR (101 MHz, $\left.\mathrm{CDCl}_{3}\right)$
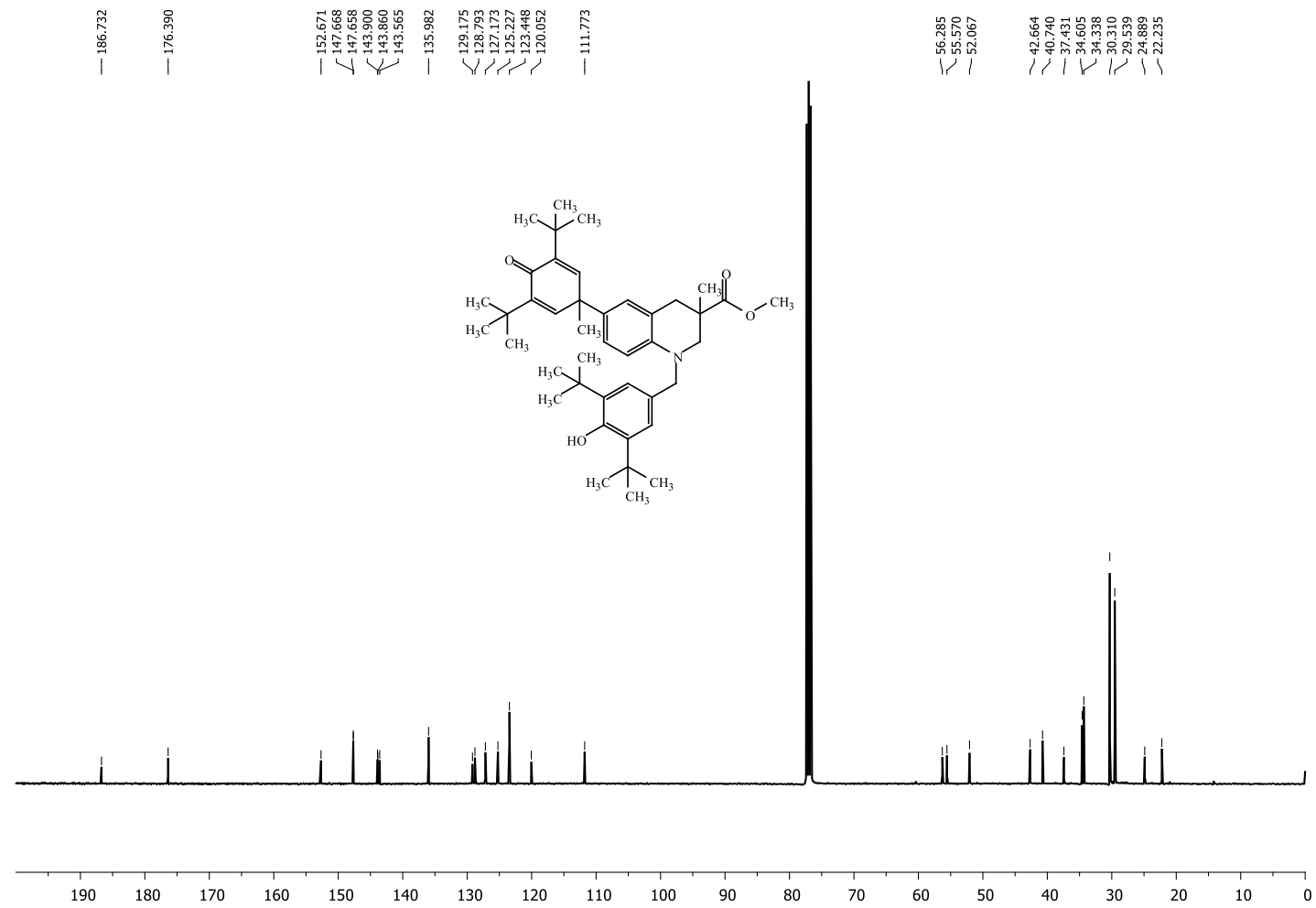

Page S102 of S104 
DEPT 135 (101 MHz, CDCl $)$
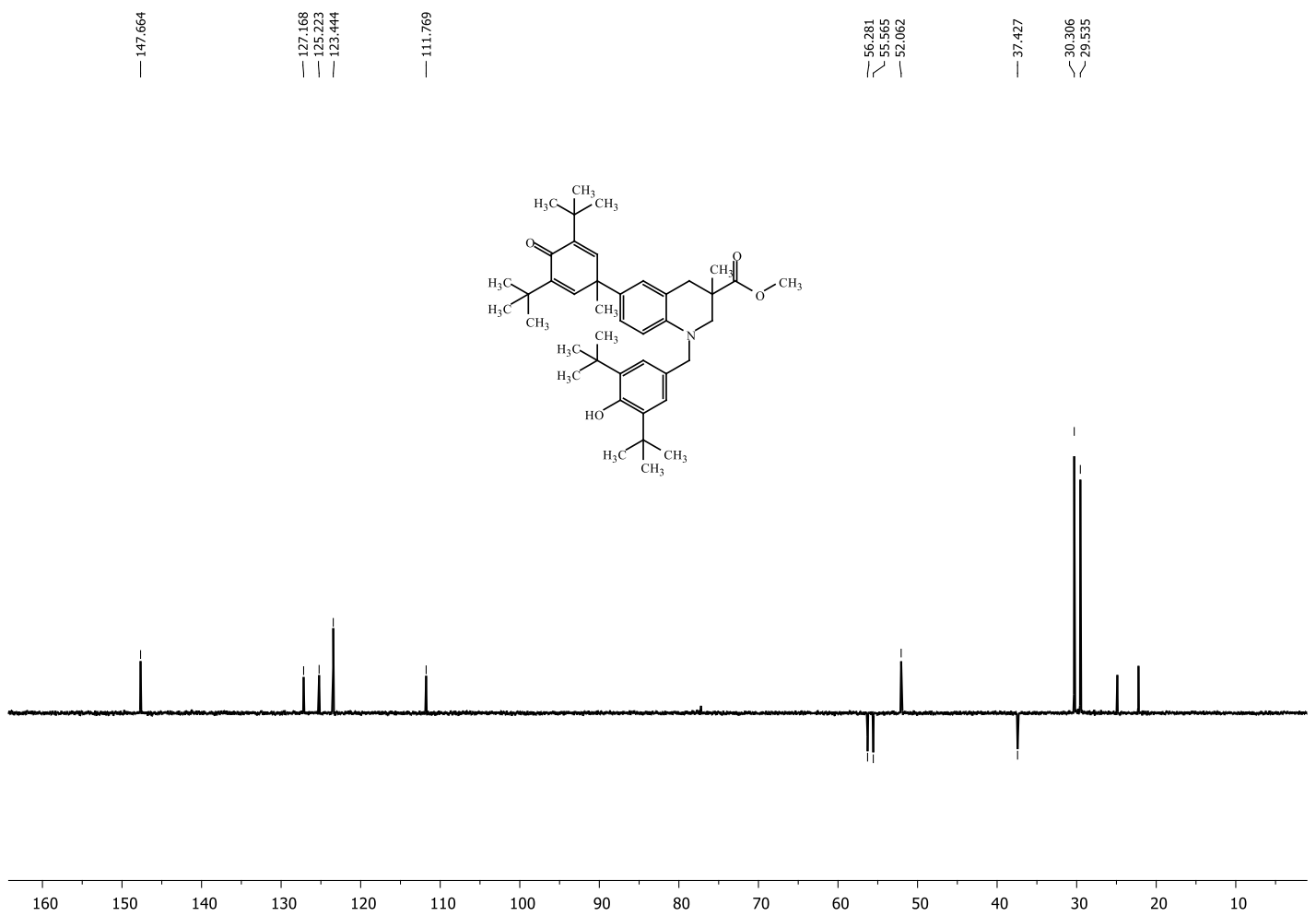

\section{COSY (400 MHz, $\left.\mathrm{CDCl}_{3}\right)$}

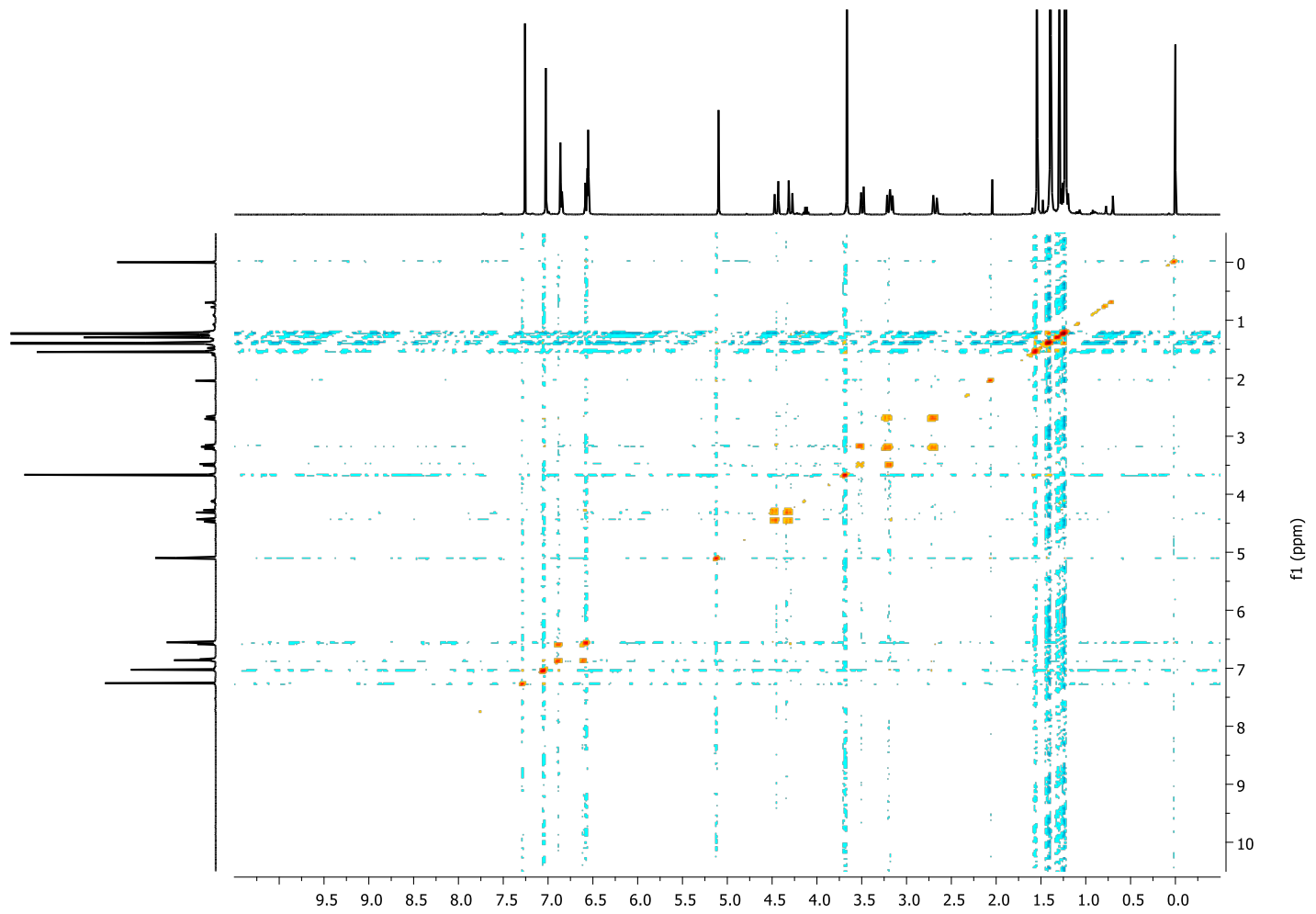




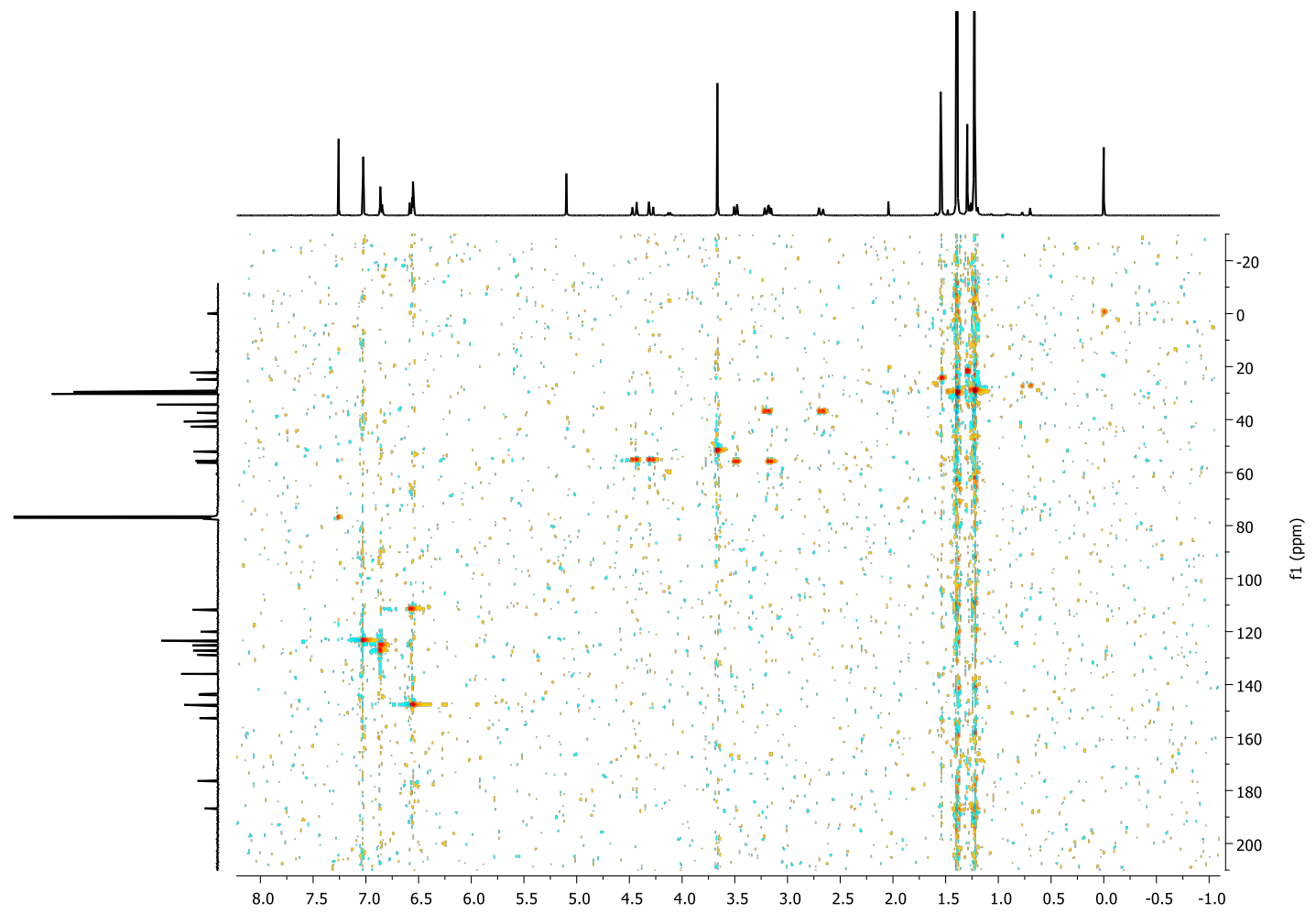

HMBC (400 MHz, CDCl 3 )

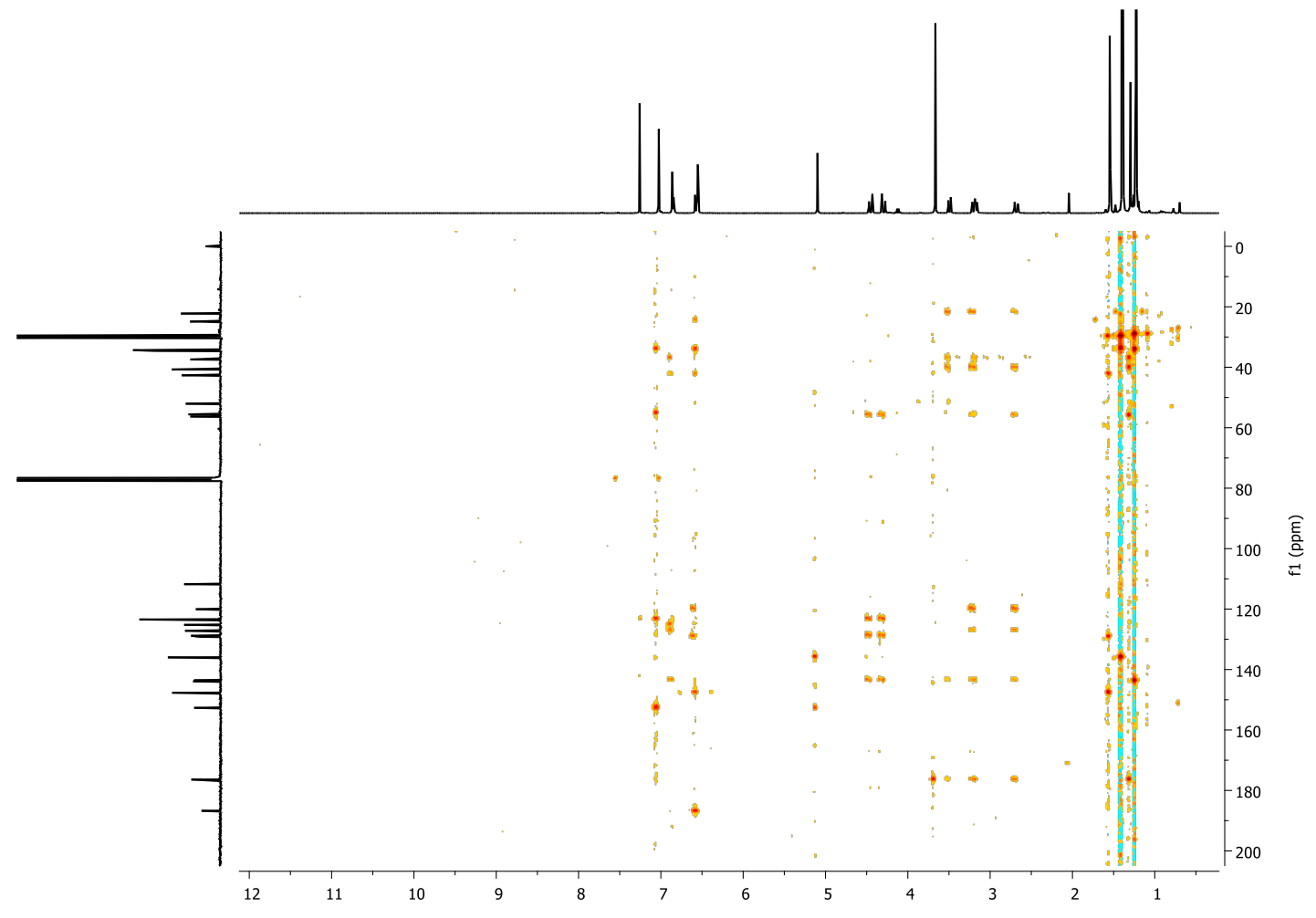

